University of Louisville

ThinkIR: The University of Louisville's Institutional Repository

Electronic Theses and Dissertations

$5-2014$

\title{
A novel numerical framework for simulation of multiscale spatio- temporally non-linear systems in additive manufacturing processes.
}

Nachiket Patil

University of Louisville

Follow this and additional works at: https://ir.library.louisville.edu/etd

Part of the Industrial Engineering Commons

\section{Recommended Citation}

Patil, Nachiket, "A novel numerical framework for simulation of multiscale spatio-temporally non-linear systems in additive manufacturing processes." (2014). Electronic Theses and Dissertations. Paper 1099. https://doi.org/10.18297/etd/1099

This Doctoral Dissertation is brought to you for free and open access by ThinkIR: The University of Louisville's Institutional Repository. It has been accepted for inclusion in Electronic Theses and Dissertations by an authorized administrator of ThinkIR: The University of Louisville's Institutional Repository. This title appears here courtesy of the author, who has retained all other copyrights. For more information, please contact thinkir@louisville.edu. 


\section{A NOVEL NUMERICAL FRAMEWORK FOR SIMULATION OF MULTISCALE SPATIO-TEMPORALLY NON-LINEAR SYSTEMS IN ADDITIVE MANUFACTURING PROCESSES}

By

Nachiket Patil

A Dissertation

Submitted to the Faculty of the

J. B. Speed School of Engineering of the University of Louisville in Partial Fulfillment of the Requirements

for the Degree of

Doctor of Philosophy

Department of Industrial Engineering

University of Louisville

Louisville, Kentucky

May 2014 
Copyright 2014 by Nachiket Patil

All rights reserved 



\title{
A NOVEL NUMERICAL FRAMEWORK FOR SIMULATION OF MULTISCALE SPATIO-TEMPORALLY NON-LINEAR SYSTEMS IN ADDITIVE MANUFACTURING PROCESSES
}

\author{
By
}

Nachiket Patil

A Dissertation Approved on

April 21, 2014

by the following Dissertation Committee:

Dissertation Director: Dr. Brent E. Stucker

Dr. Thomas L. Starr

Dr. William E. Biles.

Dr. Gail W. DePuy

Dr. Deepankar Pal 
To my late father Bapurao Patil. 


\section{ACKNOWLEDGEMENT}

I am grateful to my advisor, Dr. Brent Stucker, for his generous guidance and support during my study and research at the University of Louisville and Utah State University. I would like to thank other committee members, Dr. Thomas Starr, Dr. William Biles, Dr. Gail Depuy and Dr. Deepankar Pal for their constructive advice during my research at the University of Louisville.

I would like to express my gratitude to the staff of the Industrial Engineering Department, whose assistance and encouragement are a valuable resource for the accomplishment of my studies. I would like to express my very great appreciation to the staff of the Rapid Prototyping Center at the University of Louisville for giving professional technical support to my experiments.

I would like to thank all the fellows in my research group for their support and friendship. Special thanks go to Dr. Deepankar Pal for providing his valuable insight in formulations. I am

grateful for the assistance given by Kai Zeng, Mohammad Nikookar, Karthik Nadimpalli, Haijun Gong, Mohammad Anam and Dr. Khalid Rafi.

I would like to thank the Office of Naval Research, National Institute of Standards and Technology and Air Force Research Laboratory for funding this research at different points in time.

Above all, I thank existence and God for the strength given to complete this work. 


\title{
ABSTRACT
}

\section{A NOVEL NUMERICAL FRAMEWORK FOR SIMULATION OF MULTISCALE SPATIO- TEMPORALLY NON-LINEAR SYSTEMS IN ADDITIVE MANUFACTURING PROCESSES}

\author{
Nachiket Patil
}

April 21, 2014

\begin{abstract}
New computationally efficient numerical techniques have been formulated for multiscale analysis in order to bridge mesoscopic and macroscopic scales of thermal and mechanical responses of a material. These numerical techniques will reduce computational efforts required to simulate metal based Additive Manufacturing (AM) processes. Considering the availability of physics based constitutive models for response at mesoscopic scales, these techniques will help in the evaluation of the thermal response and mechanical properties during layer-by-layer processing in AM. Two classes of numerical techniques have been explored. The first class of numerical techniques has been developed for evaluating the periodic spatiotemporal thermal response involving multiple time and
\end{abstract}


spatial scales at the continuum level. The second class of numerical techniques is targeted at modeling multi-scale multi-energy dissipative phenomena during the solid state Ultrasonic Consolidation process. This includes bridging the mesoscopic response of a crystal plasticity finite element framework at inter- and intragranular scales and a point at the macroscopic scale. This response has been used to develop an energy dissipative constitutive model for a multi-surface interface at the macroscopic scale.

An adaptive dynamic meshing strategy as a part of first class of numerical techniques has been developed which reduces computational cost by efficient node element renumbering and assembly of stiffness matrices. This strategy has been able to reduce the computational cost for solving thermal simulation of Selective Laser Melting process by $\sim 100$ times. This method is not limited to SLM processes and can be extended to any other fusion based additive manufacturing process and more generally to any moving energy source finite element problem.

Novel FEM based beam theories have been formulated which are more general in nature compared to traditional beam theories for solid deformation. These theories have been the first to simulate thermal problems similar to a solid beam analysis approach. These are more general in nature and are capable of simulating general cross-section beams with an ability to match results for complete three dimensional analysis. In addition to this, a traditional Cholesky decomposition algorithm has been modified to reduce the computational cost of solving simultaneous equations involved in FEM simulations.

Solid state processes have been simulated with crystal plasticity based nonlinear finite element algorithms. This algorithm has been further sped up by introduction of an 
interfacial contact constitutive model formulation. This framework has been supported by a novel methodology to solve contact problems without additional computational overhead to incorporate constraint equations averting the usage of penalty springs. 


\section{TABLE OF CONTENTS}

ABSTRACT

RESEARCH MOTIVATION AND PROBLEM STATEMENT …............................................ 1

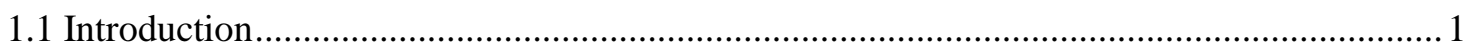

RESEARCH OBJECTIVES AND SIGNIFICANCE OF THE RESEARCH ............................. 6

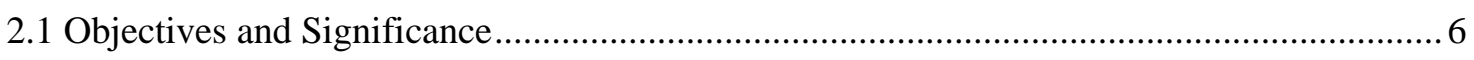

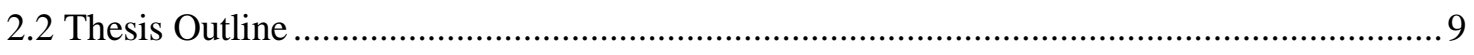

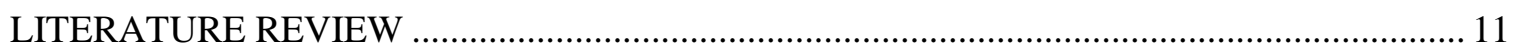

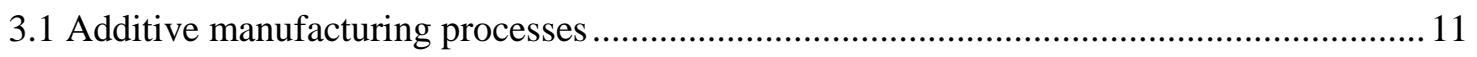

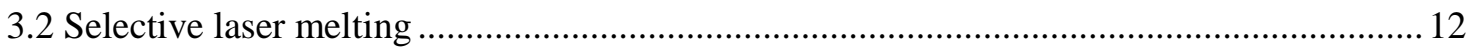

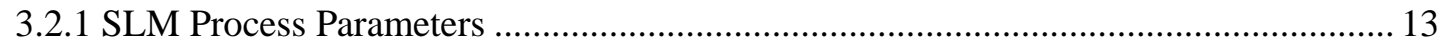

3.2.2 SLM Heat Transfer Phenomenon Description ......................................................... 14 


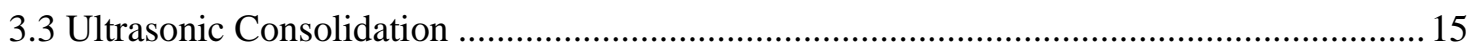

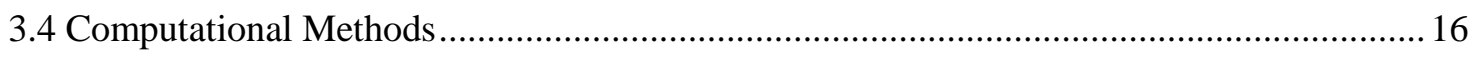

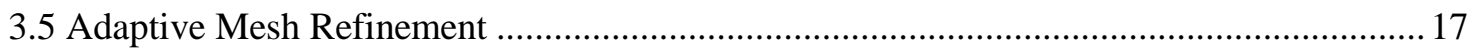

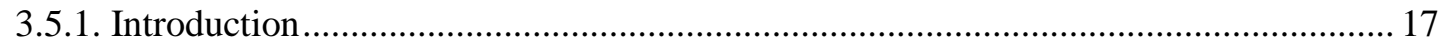

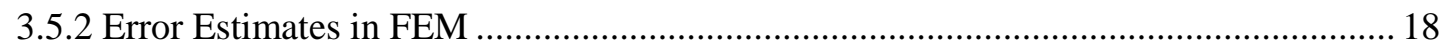

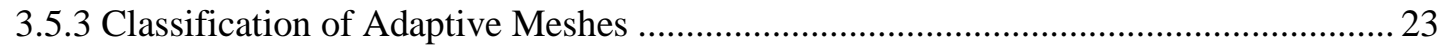

3.5.4 Data transfer between non-matching meshes with matching domain.......................... 29

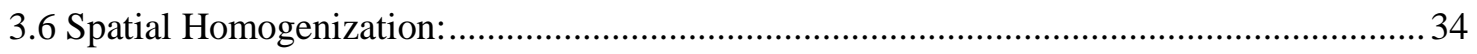

A GENERALIZED FEED FORWARD DYNAMIC ADAPTIVE MESH REFINEMENT AND DE-REFINEMENT FINITE ELEMENT FRAMEWORK FOR SPATIOTEMPORALLY PERIODIC LOCALIZED BOUNDARY CONDITIONS: APPLICATION TO METAL LASER

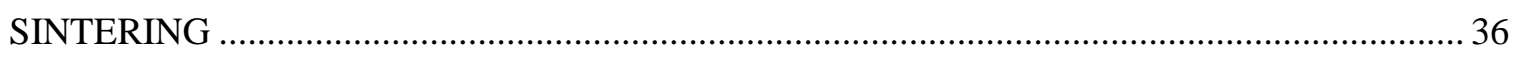

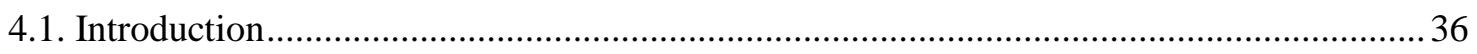

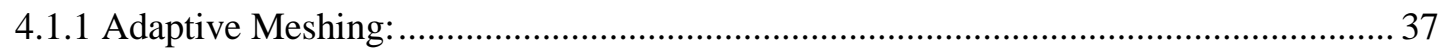

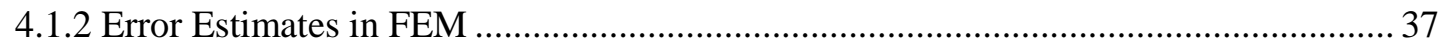

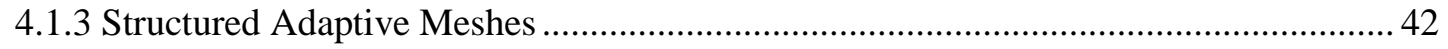

4.1.4 Adaptive Meshing in Dynamic Problems and Data Transfer between Meshes ............ 44

4.1.5 Spatio-temporal Periodic Problems and Metal Laser Sintering .................................. 47

4.1.6 Governing Equations and Finite Element Formulation in FFD-AMRD ...................... 48

4.2 FFD-AMRD framework for applications in spatio-temporally periodic localized boundary

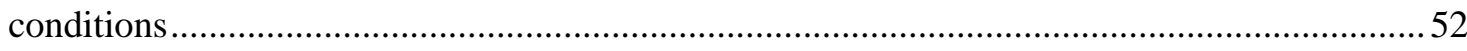


4.2.1 Fast Sorting Methodologies to Increase Data Transfer Between Meshes

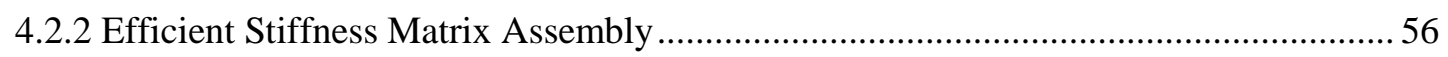

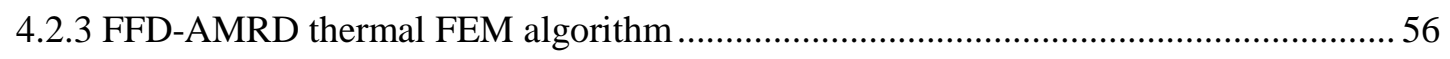

4.2.4 Comparison of FFD-AMRD algorithm with traditional FEM .................................... 62

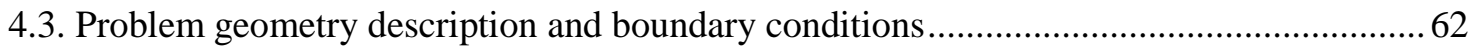

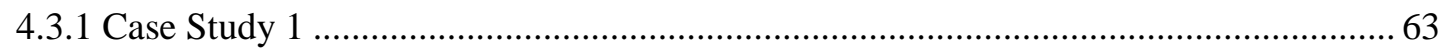

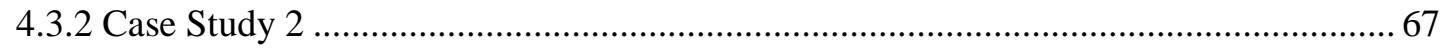

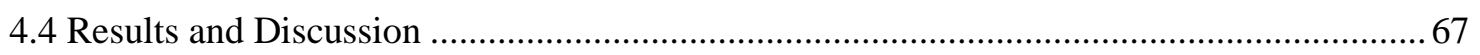

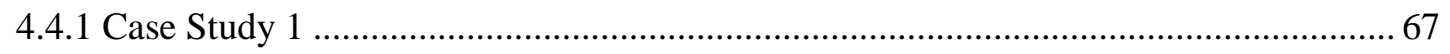

4.4.2 Three dimensional FEM description of spatio-temporally periodic metal laser sintering

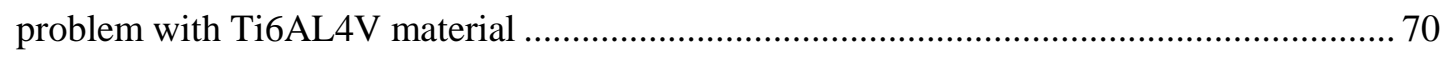

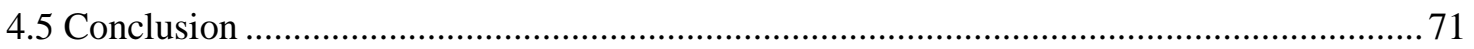

A NEW FINITE ELEMENT SOLVER USING NUMERICAL EIGEN MODES FOR FAST SIMULATION OF ADDITIVE MANUFACTURING PROCESSES ...................................... 72

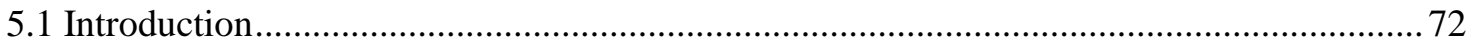

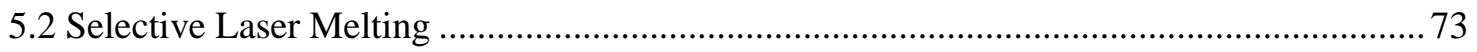

5.3 Governing equations and boundary conditions........................................................ 74

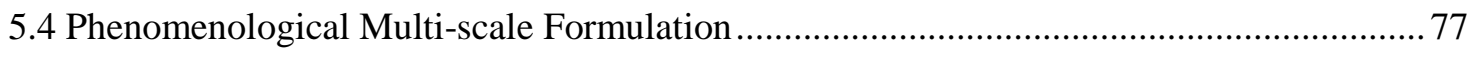

5.4.1 FFD-AMRD simulation covering fine-scale and fine to coarse scales:....................... 79

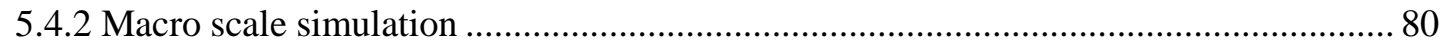

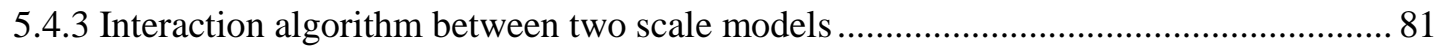




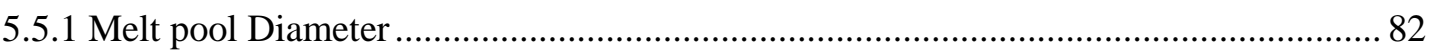

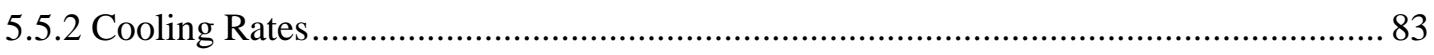

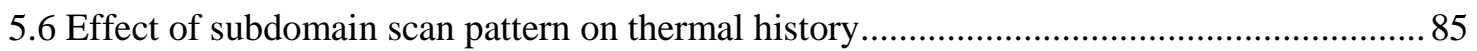

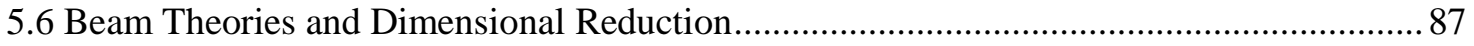

5.6.1 Dimensional Reduction for a Prismatic Powder Bed.................................................. 88

5.6.2 Modal Space based Beam Theories Formulation......................................................... 89

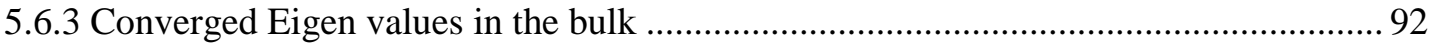

5.6.4 Eigen Modes for the prismatic powder bed: case study ........................................... 93

5.7 Extension of the present mathematical formulation to geometry with dissimilar layers.....94

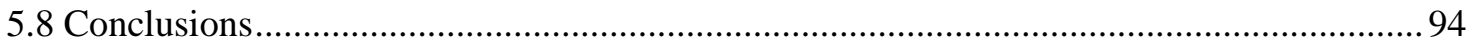

INTELLIGENT CHOLESKY DECOMPOSITION FOR EXCLUDING THE COMPUTATIONAL OVERHEAD OF INSIGNIFICANT MATRIX MULTIPLICATIONS .... 96

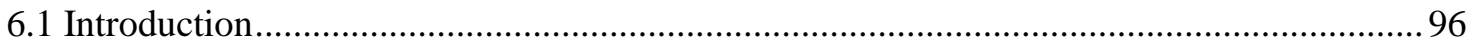

6.1.1 Variants of Cholesky Decomposition in Literature ..................................................... 97

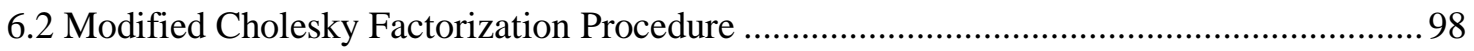

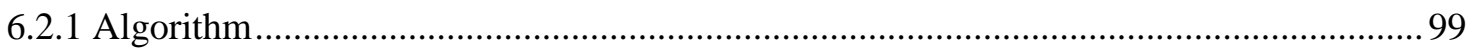

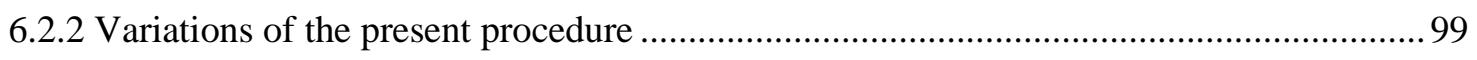

6.3 Case Study: Steady state thermal analysis with intelligent Cholesky algorithm .............. 102

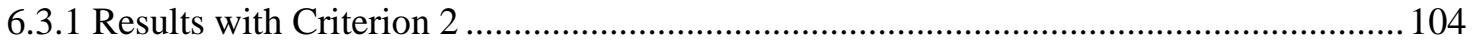

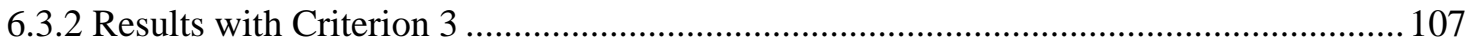


6.5 Conclusions.

AN ENERGY DISSIPATIVE CONSTITUTIVE MODEL FOR MULTI-SURFACE INTERFACES AT WELD DEFECT SITES IN ULTRASONIC CONSOLIDATION 111

7.1 Introduction. 111

7.2 Ultrasonic Consolidation 111

7.3 Interfacial Constitutive Model 113

7.3.1 Elastic plastic interfacial constitutive modeling Literature [137] 114

7.4 Applications of the interfacial constitutive models.

7.5 Mathematical Formulation 117

7.5.1Contact Surface Constraints and solution of constrained simultaneous equations

7.6 Contact Simulation Algorithm 121

7.7 Case Study 122

7.8 Results and Discussion 124

7.9 Conclusions and future work 126

A NOVEL CRYSTAL PLASTICITY AND SURFACE TO SURFACE CONTACT SIMULATION FRAMEWORK FOR SIMULATION OF ADDITIVE MANUFACTURING PROCESSES 128

8.1 Introduction. 128

8.2 Plastic deformation and FEM implementation

8.3 Stress Jacobian Derivation. 131

8.4 Nonlinear Finite Element Algorithm 134 


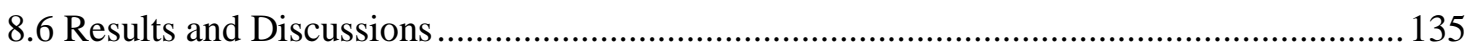

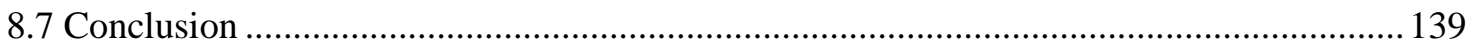

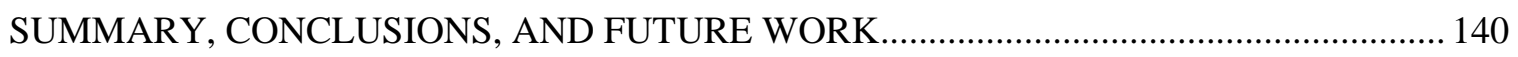

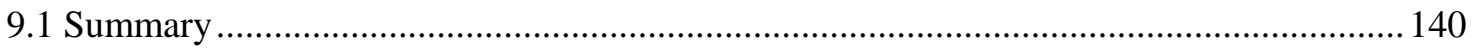

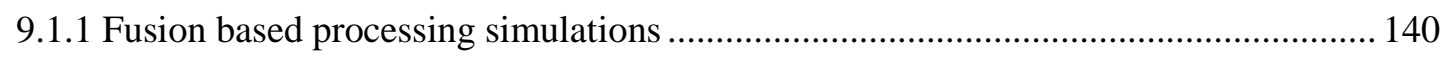

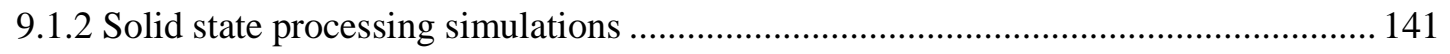

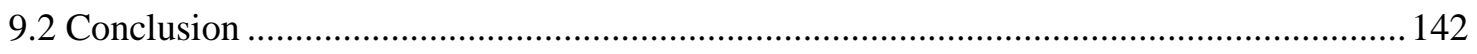

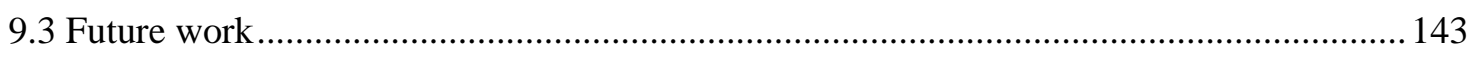

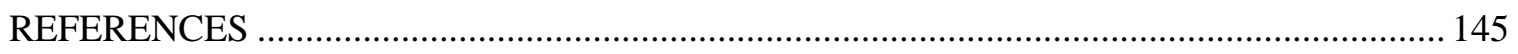

A GENERALIZED FEED FORWARD DYNAMIC ADAPTIVE MESH REFINEMENT AND DE-REFINEMENT FINITE ELEMENT FRAMEWORK FOR METAL LASER SINTERING: PART II (NON-LINEAR THERMAL SIMULATIONS AND VALIDATIONS) ..................... 162

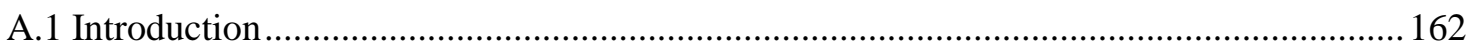

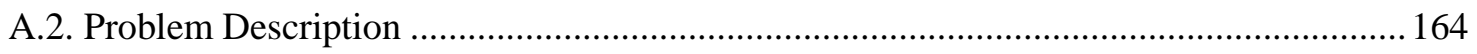

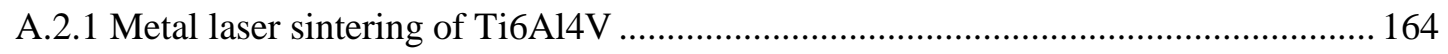

A.2.2 Asymptotic approximation for localized spatio-temporally periodic boundary condition

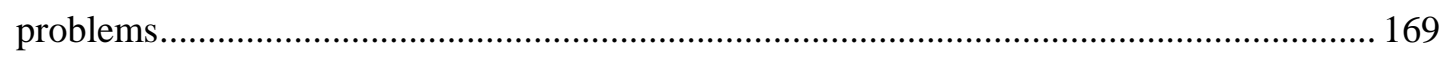

A.2.3 Locally enriched Dynamic Mesh (FFD-AMRD framework)[162] ........................... 172

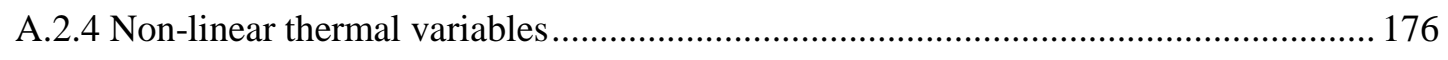

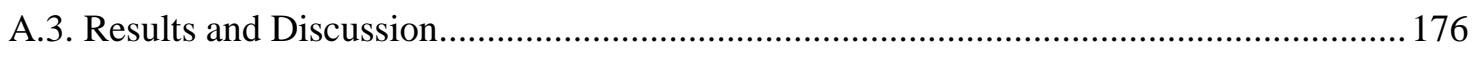

A.3.1 Three dimensional spatio-temporally periodic FEM results .................................... 176 
A.3.2 Thermal and microstructural validations

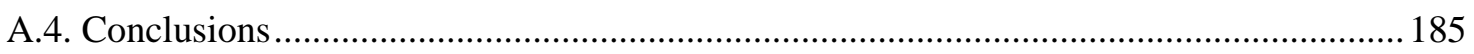

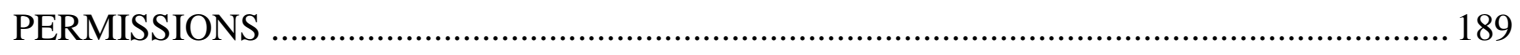

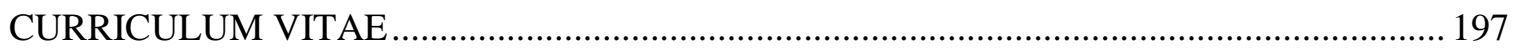




\section{LIST OF TABLES}

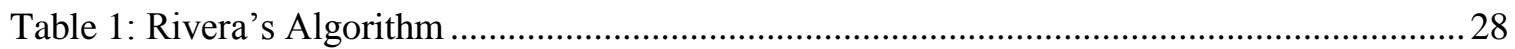

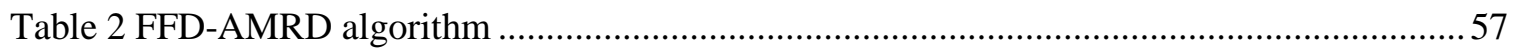

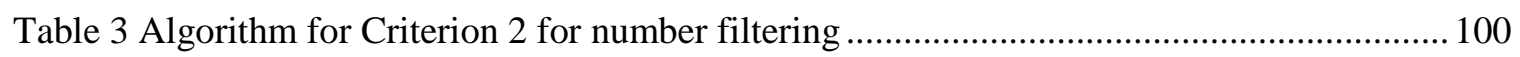

Table 4 Plastic deformation finite element algorithm with crystal plasticity based constitutive

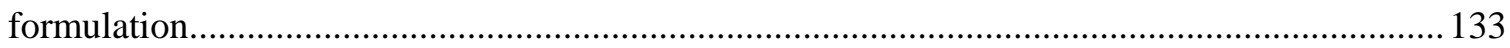




\section{LIST OF FIGURES}

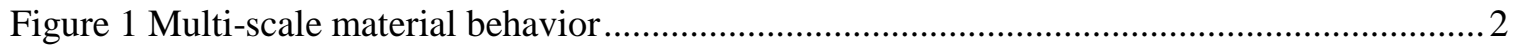

Figure 2 Schematic diagram showing heat transfer phenomena in an SLM process [30] ............ 13

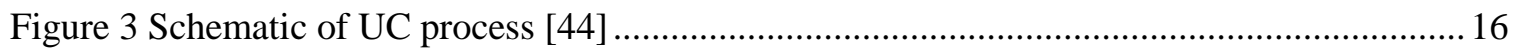

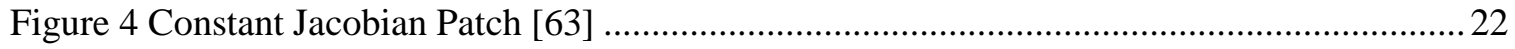

Figure 5 (a) Geometry and (b) data structure of a 3-level mesh tree. [84]..................................24

Figure 6 Mesh strategy for block structured refinement [67] ...................................................24

Figure 7 Adaptive Mesh with Mesh Distortion [65] ..............................................................25

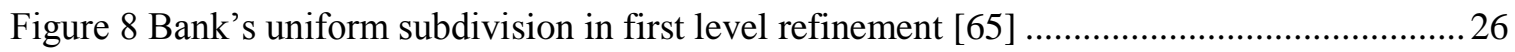

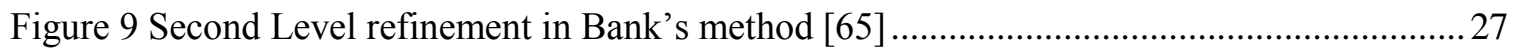

Figure 10 First two iterations of Rivera's Bisection of the longest side of a triangle [65] ...........27

Figure 11 Coarsening of a triangulation by Edge Collapsing [65] .............................................29

Figure 12 Example triangulation where collapsing a vertex can lead to an erroneous mesh [65].29

Figure 13 Global Coordinate System and Isoparametric Element ............................................. 31

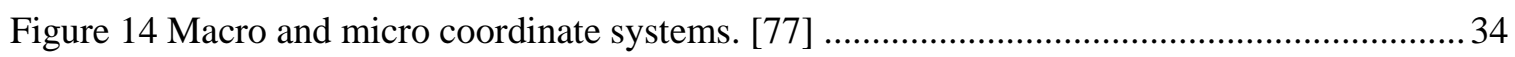

Figure 15 Definition of periodic boundary conditions: (a) initial unit cell and (b) deformed unit cell.

Figure 16: (a) Geometry and (b) data structure of a 3-level mesh tree [84]. ............................... 43

Figure 17 An example of a three-level, block-structured AMR hierarchy [86]........................... 43

Figure 18 Adaptive Mesh with Mesh Distortion .................................................................... 44

Figure 19 Schematic Diagram showing FFD-AMRD dynamic mesh ......................................49 
Figure 20 A brick element with 8 integration points. The $B$ integration scheme has been incorporated .50

Figure 21 Schematic showing old (blue elements) and next (red nodes) mesh configurations.....54

Figure 22 Target Element (old mesh configuration) for illustrated nodes (next mesh configuration).

$\mathrm{x}$ and $\mathrm{y}$ bounds are shown with local isoparametric target element axes $\zeta$ and $\varepsilon \ldots \ldots \ldots \ldots \ldots \ldots \ldots \ldots . . . . . . . . . . . . . .54$

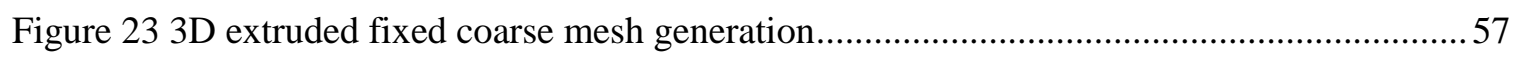

Figure $243 \mathrm{D}$ extruded independent fine mesh generation ....................................................5

Figure 25 FFD-AMRD mesh, stiffness and specific heat generation matrix ...............................59

Figure 26 Dynamic boundary condition of the one-dimensional problem .................................63

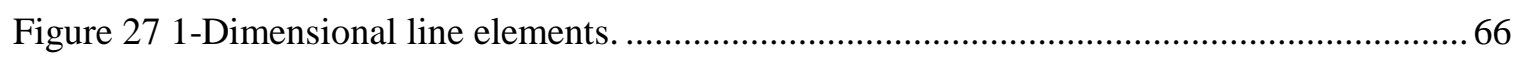

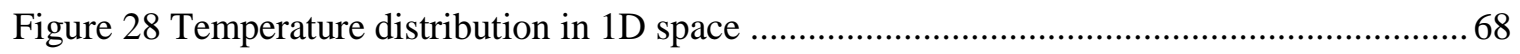

Figure 29 Thermal conductivity distribution for nonlinear and linear cases of the one dimensional problem at three different times plotted against nodes.

Figure 30 Volumetric heat capacity $(\rho c)$ for nonlinear and linear cases of the one dimensional problem at three different times plotted against nodes

Figure 31 Comparison of thermal contours. The melt pool diameter in (b) is $125 \mu \mathrm{m}$. 70 Figure 32 Optical microscopy image of microstructure showing grain boundaries along the melt pool boundary. .71

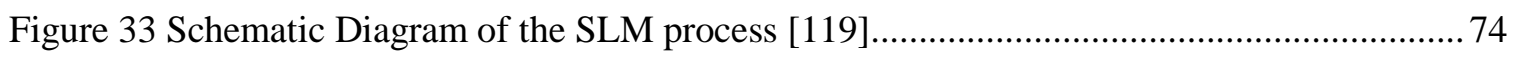

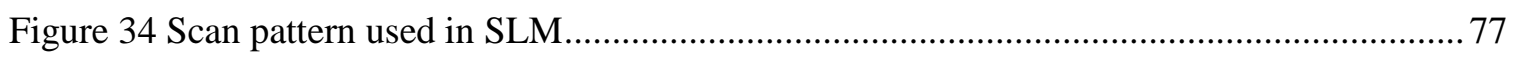

Figure 35 Multi-scale analysis framework for simulation of the SLM process............................78

Figure 36 Interaction between two scale models ................................................................ 81

Figure 37 : (a) Melt pool diameter variation during a subdomain hatch for the case with $750 \mathrm{~K}$ initial temperature. (b) Effect of initial global temperature on melt pool in SLM of stainless steel powder. 
Figure 38 Cooling rates at different initial powder bed temperatures

Figure 39 Powder bed cooling down through global mode of uniform average temperature. The zoomed in picture shows the local fast temporal variation of temperature due to laser beam inside fine to coarse model. 84

Figure 40 (a) Scan pattern used for two successive subdomains scan (b) Thermal profile at particular time in the prior subdomain scan. .86

Figure 41 Maximum Temperature comparison in two cases (with and without cooling time between successive scans) 87

Figure 42 Figure 10: Problem geometry and FEM mesh. 89

Figure 43 First four significant modes for of the powder bed case study c/s .92

Figure $445^{\text {th }}$ to $8^{\text {th }}$ significant modes for of the powder bed case study c/s .93

Figure 45: Algorithm to determine which values in the E matrix have information which are significant. 101

Figure 46: Problem geometry and boundary conditions 103

Figure 47: Comparison between standard Cholesky versus Intelligent Cholesky algorithm presented with criterion 2 .

Figure 48 (a) Cholesky matrix computed through the intelligent Cholesky algorithm. (b) Comparison of temperatures computed using intelligent Cholesky and Matlab Cholesky at a threshold of 80 105 Figure 49 (a) Cholesky matrix computed using the intelligent Cholesky algorithm and (b) Comparison of temperatures computed using intelligent Cholesky and Matlab Cholesky algorithms at a threshold of 160 . 106 Figure 50: Figure (20). Comparison between standard Cholesky versus Intelligent Cholesky algorithm with criterion 3 . 107 
Figure 51 A typical row of the Cholesky decomposition matrix. (b) Fourier transform of the typical

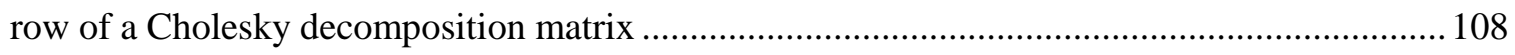

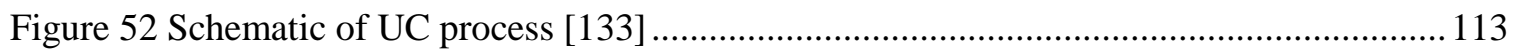

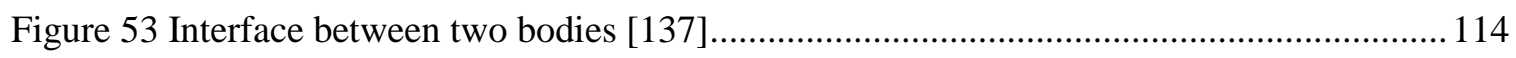

Figure 54 Schematic of the yield surface for normal and shear mechanisms [137].................. 116

Figure 55: Schematic diagram presenting the concept of an interfacial constitutive model for a

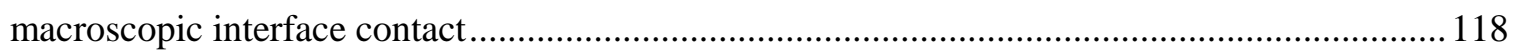

Figure 56 Schematic diagram representing a slave node constraint on a contact surface and its solution methodology by splitting slave node stiffness as per the constraint equation................ 121

Figure 57 Flow chart showing FEM algorithm used for contact simulation ............................. 122

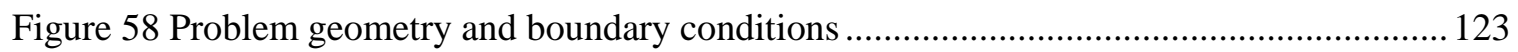

Figure 59: Macroscopic strain versus shear resistance plot ..................................................... 124

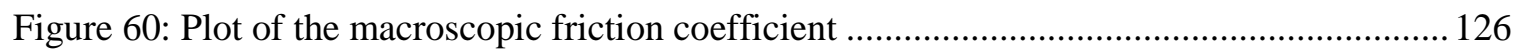

Figure 61: Deformed and un-deformed mesh at a macroscopic shear strain of $0.05 \ldots \ldots \ldots \ldots \ldots \ldots . . . .127$

Figure 62 Schematic representing (a) microstructure global axis and (b) the grain axis [140]... 130

Figure 63 Schematic diagram showing elastic loading followed by plastic unloading ............... 132

Figure 64 Plot of stress vs strain for the case of monotonic uniaxial tensile loading .................136

Figure 65 plot of $C 33$ (indicator of elastic modulus in the z-direction) vs time for the case of

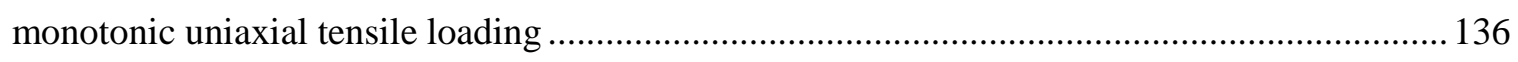

Figure 66 plot of Fp33 (Elastic modulus in the z-direction) vs time for the case of monotonic uniaxial tensile loading 137

Figure 67 Average stress vs time plot for the case of elastic-plastic loading followed by relaxation 137

Figure 68 plot of $C 33$ (indicator of elastic modulus in the z-direction) vs time for the case of elasticplastic loading followed by relaxation 138 
Figure 69 plot of Fp33 (Elastic modulus in the z-direction) vs time for the case of elastic-plastic

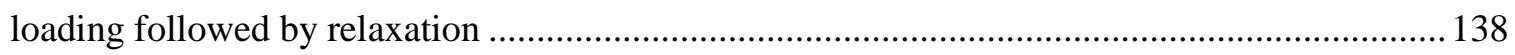

Figure 70 Plot of various quantities of interest in interface contact simulation with plasticity ... 139 


\section{CHAPTER 1 \\ RESEARCH MOTIVATION AND PROBLEM \\ STATEMENT}

\subsection{Introduction}

Natural phenomena have been scientifically classified into four fundamental interactions [1]: gravitation, electromagnetism, strong nuclear and weak nuclear. These interactions have different manifestations at different scales of a material. Every interaction has multi-scale effects if the body geometry has significant length available for its spatial evolution.

Fig 1 shows a multi-scale material structure for any crystalline material and relevant length scales. Atoms or molecules can be considered as the fundamental building blocks from the perspective of ability to tweak properties to engineer macroscopic material properties. Multiple atoms stack together in a particular pattern to form a crystal structure [2]. The manufacturing process has a decisive impact on the crystal structure of the fabricated part. These crystals demonstrate [3] certain point, line, area and volume defects when the crystallite attains a particular length scale. Point defects such as interstitial defects are important from the perspective of alloying while interacting with the perfect crystal and higher dimensional defects. Some examples of line, area and volume defects are 

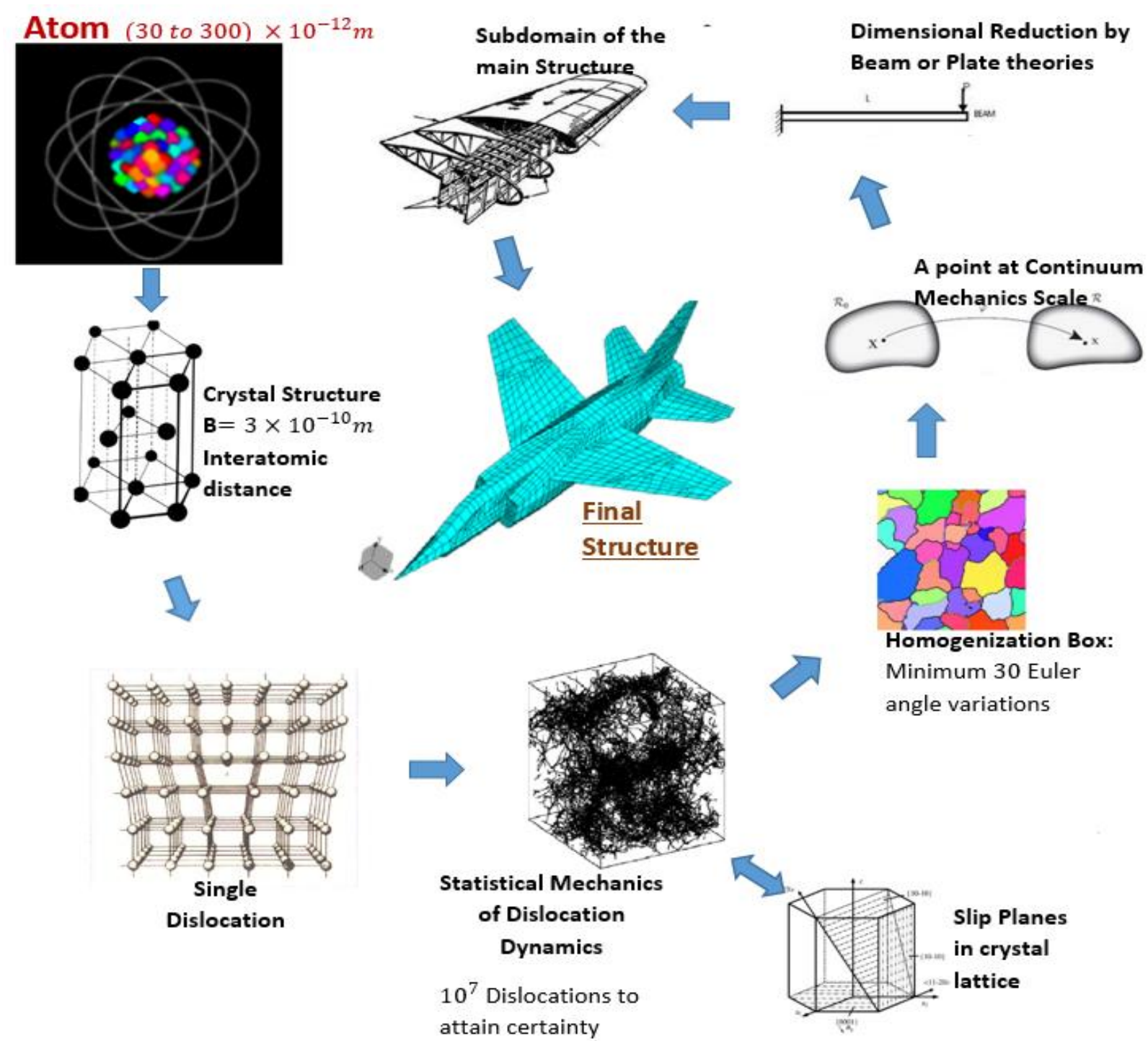

Figure 1 Multi-scale material behavior

dislocations, grain boundaries and precipitates respectively. These defects interact with each other leading to the determination of response to external stimuli. The vibrational uncertainty [4] at the atomic level has some implications for behavior of a single dislocation, and this can be quantified with molecular dynamics simulations for small time frames. These models are difficult to extend further in space and time considering the involved computational cost and numerical error propagation. 
The vibrational uncertainty associated with a dislocation is further augmented by configurational uncertainty [5] of dislocation interactions. The combined uncertainty at the scale of atomic and dislocation cluster interactions leads to multi-scale stochastic behavior of the underlying material and calls for remedies using statistical mechanics [6]. The constitutive model developed with the help of statistical mechanics can be used as a point behavior law for mesoscopic continuum mechanics simulations.

The mesoscopic continuum mechanics constitutive model developed for the framework described above needs a mathematical framework, for example homogenization [7], which can consider microstructure and phase information to numerically formulate a macroscopic material constitutive law. This constitutive law for each point along with an equilibrium equation [8] results in a governing partial differential equation (PDE). These PDEs can be solved analytically for very simple geometries and require use of numerical techniques like Finite Element Method (FEM) [9]. These numerical methods rely on discretization of geometry and reducing the PDE into sets of linear equations. One of the major limitations of these numerical methods is numerical cost when dealing with problems involving significant spatial-temporal variations along with complicated geometries and boundary conditions.

Material constitutive laws, geometry and time dependent boundary conditions can together determine the fabricated part performance in service. The multiscale material nature discussed above along with manufacturing freedom can be optimized together to produce a highly efficient product with good mechanical properties and serviceability. Various traditional manufacturing methods have limitation with respect to geometrical 
freedom. Considering this limitation, Additive Manufacturing [10] (AM) has emerged as a new technology with immense new possibilities for freeform fabrication.

There are many metal based Additive Manufacturing processes. Two categories [10] that will be explored in this work are laser based powder bed melting and solid state welding. Respective example processes [10] for these categories are Selective Laser Melting (SLM) and Ultrasonic Consolidation (UC). Simulation of these processes can help with better quantification of the physics involved, and this quantification can further be employed to better control machines for different material and part geometry scenarios. The present state of work in this direction has found a bottleneck at the level of solving PDEs at mesoscopic and macroscopic scales.

In this work numerical techniques are attempted for bridging mesoscopic and macroscopic scale variations involved in representative Additive Manufacturing processes. This work overcomes the present bottlenecks in simulation of these processes such as the computational cost needed to simulate high heating and cooling rates involved in the thermal simulation of SLM (spatio-temporally periodic phenomena) and the high strain rate and non-conservative energy dissipation mechanisms in UC.

SLM thermal behavior [11] is important from the perspective of predicting phases and microstructure in the built part based on cooling rates after melting using the laser heat source. The laser heat source moves over the powder bed at speeds on the order of $2 \mathrm{~m} / \mathrm{s}$ and cooling rates above $10^{5} \mathrm{C} / \mathrm{s}$. Simulation of this scenario with finite element methods requires very fine mesh refinement to capture spatial variations near the laser beam (mesh refinement must be much smaller than the laser beam diameter of $\sim 100 \mu \mathrm{m})$. This thermal 
behavior has multiple scales both in space and time and it is computationally very expensive to solve with traditional numerical methods.

In comparison with SLM, UC is a solid state process [5] in which two thin foils of metal are joined by applying ultrasonic vibration in addition to a normal force in order to break the oxide layers on foil surfaces and create an inter-atomic bond. In this process it has been seen that the quality of the bond and linear weld density are dependent on the amount of ultrasonic energy supplied to the interface to break the oxide layer and induce plastic deformation. The amount of energy that is transferred to the interface for this purpose is a function of the overall geometry of the partially built part and the inter-laminar defects present in the build. These defects cause energy dissipation and their exact quantification requires modeling the friction and plastic deformation on surfaces of both foils. This phenomenon has a smaller scale compared to macroscopic bulk deformation. Visco-plastic deformation, which is the reason for energy dissipation, is dependent on the microstructure. Microstructure dependence of the visco-plastic deformation constitutive law can be attained numerically using homogenization techniques.

If these two processes and their corresponding multi-scale simulation obstacles can be solved, 'manufacturing integrated' optimization of numerous day to day engineering products can result. Further this will lead to a better numerical framework for solving various multiscale phenomena discussed at the start of this chapter. 


\section{CHAPTER 2}

\section{RESEARCH OBJECTIVES AND SIGNIFICANCE OF}

\section{THE RESEARCH}

\subsection{Objectives and Significance}

A muliscale simulation framework for additive manufacturing processes has been attempted in the present work with an emphasis on development of numerical algorithms with reduced computational cost. The computational complexity has been related to a set of governing partial differential equations and the numerical algorithms employed to solve them for given boundary conditions. The present work has been focused at formulating new numerical algorithms taking advantage of process specific multiscale behavior involved in these processes. The generic or broader research objectives for this research work are as follows

1. Development of an efficient adaptive finite element framework for simulation of spatiotemporally periodic problems such as selective laser melting which will involve a dynamic fine mesh region in a course mesh to capture high gradients at the point of exposure or near the melt-pool. This algorithm should be efficient enough to reduce the computational cost by $10 \mathrm{X}$ to $100 \mathrm{X}$ depending on problem size and input processing parameters. 
2. Development of a new visco-plastic continuum constitutive model for energy dissipation mechanisms such as friction at mating interfaces in UC. This will involve homogenization of the micro-structural response using statistical mechanics based crystal plasticity constitutive models [5]. This algorithm is efficient enough to reduce the computational cost by $5 \mathrm{X}$ to $20 \mathrm{X}$ depending on problem size and input processing parameters.

3. Development of high efficiency methods for storage and solution of FEM matrices in problems involving prismatic structures, such as powder beds and part layers for thermal simulation of SLM.

The successful completion of this research project based on the above objectives will contribute significantly to the following research areas in multi-scale analysis and Additive Manufacturing

- It will result in efficient algorithms for adaptive refinement and derefinement of FEM meshes for applications in problems with spatiotemporally periodic boundary conditions or responses which have rapid variations in time and space. This will also be helpful for bridging multiple scales of deformation present within a continuum scale problem for cases of elliptical and parabolic partial differential equations.

- A new visco-plastic continuum constitutive model for interface friction and its energy dissipation mechanism in UC will be formulated. This novel interface constitutive law will reduce the computational cost of modeling interfaces while modeling bulk response with a significantly simplified 
mesh and reduced computational cost. Rolling and forming process simulations or friction based dampers are additional potential applications.

- An efficient bridge between mesoscopic scale statistical mechanics based crystal plasticity constitutive response and macro-scale visco-plastic deformation in metals will be developed. This will be useful for highfidelity analysis of metal parts for process analysis as well as life cycle performance analysis of manufactured product.

- Efficient methods for storing and solving FEM matrices in cases of prismatic structures, for example in powder beds and part layers for thermal simulation of SLM. This will be useful in analysis and design of various structures and processes involving simple geometry with layered structures (such as composite laminates).

The expected implications and impact of the present work will be as follows.

1. A multi scale simulation framework 10 to $100 \mathrm{x}$ faster than traditional simulation frameworks for simulating additive manufacturing processes along with reduced computational memory (random access memory) requirements.

2. Better process optimization through reduced computational complexity tools.

3. Prediction of part mechanical properties such as plastic softening or hardening, part mechanical strength and fatigue life. 


\subsection{Thesis Outline}

This thesis is written in the multi-paper format, where each chapter represents a standalone conference paper or journal article The available literature on relevant numerical methods are discussed in detail in Chapter 3, but Chapter 3 is not intended to be published as a stand-alone article outside this dissertation.

Chapter 4 involves development of a numerical Finite Element algorithm for thermal simulation of SLM. SLM simulation requires a multi scale FEM framework with spatiotemporal adaptive meshing where the fine mesh domain moves along with the laser scan path. This will also involve an intelligent algorithm which can refine and de-refine the fine mesh near the laser spot. The process of refinement and de-refinement requires renumbering and recalculation of the FEM matrices and this has been addressed with formulation of an efficient algorithm in order to harness the benefits of this spatiotemporal adaptive meshing strategy. The computational cost reduction through the two scale FEM model presented in this chapter needs further improvements for simulation of full powder bed simulations. This paper is in review with Journal of Manufacturing Science and Engineering.

Chapter 5 presents a three scale simulation framework with a novel Eigenmode based strategy coupled with FFDAMRD to simulate the full powder bed problem. Linear equation solvers are required for the computational algorithms used in FFDAMRD, Eigenmodes, and for general FEM simulations. This paper has been published in the Solid Freeform Fabrication Symposium for 2013. 
Chapter 6 presents a novel methodology to reduce the computational cost for solving positive definite simultaneous equations using Cholesky Decomposition. This paper is not yet published and will be submitted to the Finite Elements in Analysis and Design Journal.

Algorithms developed in Chapter 3 and 4 have been focused on fusion based processes. Solid state processes in additive manufacturing such as UC involve interfacial contact simulations. Chapter 7 includes formulation of an efficient algorithm to simulate contact problems along with a novel methodology to solve constraint equations with reduced computational cost. This algorithm formulates an interface contact constitutive relation for linear elastic materials. This paper has been published in the Solid Freeform Fabrication Symposium for 2013.

A more accurate material representation for development of the constitutive model developed in Chapter 7 is shown in Chapter 8. This chapter shows classical crystal plasticity simulations and has future potential for solving dislocation density based crystal plasticity along with spatial homogenization. This paper is not yet published and will be submitted to the Finite Elements in Analysis and Design Journal.

Appendix A shows a Nonlinear version of the FFD-AMRD algorithm presented in Chapter 4. The nonlinearity considered here has been due to temperature dependent thermal properties of solidified part, molten metal and powder. This paper has been submitted to the Journal of Manufacturing Science and Engineering and is currently under review. 


\section{CHAPTER 3}

\section{LITERATURE REVIEW}

\subsection{Additive manufacturing processes}

Additive Manufacturing (AM) processes are manufacturing processes in which complex 3-dimesional solid shapes based on a CAD model are manufactured layer by layer to the desired geometry. This technology has distinct advantages over traditional manufacturing in its capability to manufacture freeform shapes without any use of molds. AM has been called by many other names, including solid freeform fabrication (SFF), rapid prototyping (RP), layered manufacturing (LM) and direct digital manufacturing (DDM). The various processing technologies categories as per ASTM F2792 - 12a [12] include binder jetting, directed energy deposition, material extrusion, material jetting, powder bed fusion, sheet lamination, and vat photopolymerization. The common feature of these processes is the numerical decomposition of the CAD model using surface triangulation in the form of a STereoLithography (STL) file. STL files are further cut into slices and are used by machines to program layer deposition. The major differences between AM processes are: (1) materials used, and (2) part building technique [13-14]. AM technologies have been widely used for the fabrication of prototypes, as one of the design and development stages for product manufacture. Other applications include rapid tooling (RT) [15-16], repair of damaged mechanical components [17], medical implants, device 
fabrication [18-21], and for other end-use functional components. The abilities of AM to fabricate complex objects without the use of molds offer designers opportunities for novel designs that would otherwise have been impossible with traditional manufacturing techniques. High levels of geometrical complexities can now be designed for manufacture with little or no restrictions for most AM technologies. AM also offers a wide range of possibilities including fabrication of structures with spatial material heterogeneity, direct build of multi-component assemblies, and the fabrication of materially graded structures in density and composition [13-19]. There is the potential to place materials just where they are needed with AM technologies.

\subsection{Selective laser melting}

Laser Sintering (LS) is a process in which a moving high energy laser beam scans the surface of a powder bed (the powder can be metal, polymer or ceramics) and the melted powder solidifies to form the bulk part. Selective Laser Melting (SLM) is the term commonly applied to versions of laser sintering with utilize powdered metal as the build material. Other nomenclatures used by machine manufacturers for this technology are Laser Cusing and Direct Metal Laser Sintering (DMLS) [22]. Selective laser melting is very complicated because of its fast laser scan speeds and material transformations in a very short timeframe. The temperature field was found to be transient by many previous researchers [23-27]. Meanwhile, the temperature evolution history in laser sintering has significant effects on the quality of the final parts, such as density, dimensions, mechanical properties, microstructure, etc. For metals, large thermal gradients increase residual stresses and deformation, and may even lead to crack formation in the fabricated part. Thermal distortion of the fabricated part is one serious problem in SLM [28]. Therefore, 
understanding the process mechanisms and effects of process parameters are significant for the future development of SLM.

\subsubsection{SLM Process Parameters}

Like any processes or methods, theoretical and experimental tests are necessary to certify and qualify SLM technology. Some of the first experiments [29] with SLM included direct sintering of bronze powder without polymer binders or preheating. It was found that process parameters and material features such as laser beam power, scanning speed, hatch distance and particle size distribution have an important influence on the melting behavior.

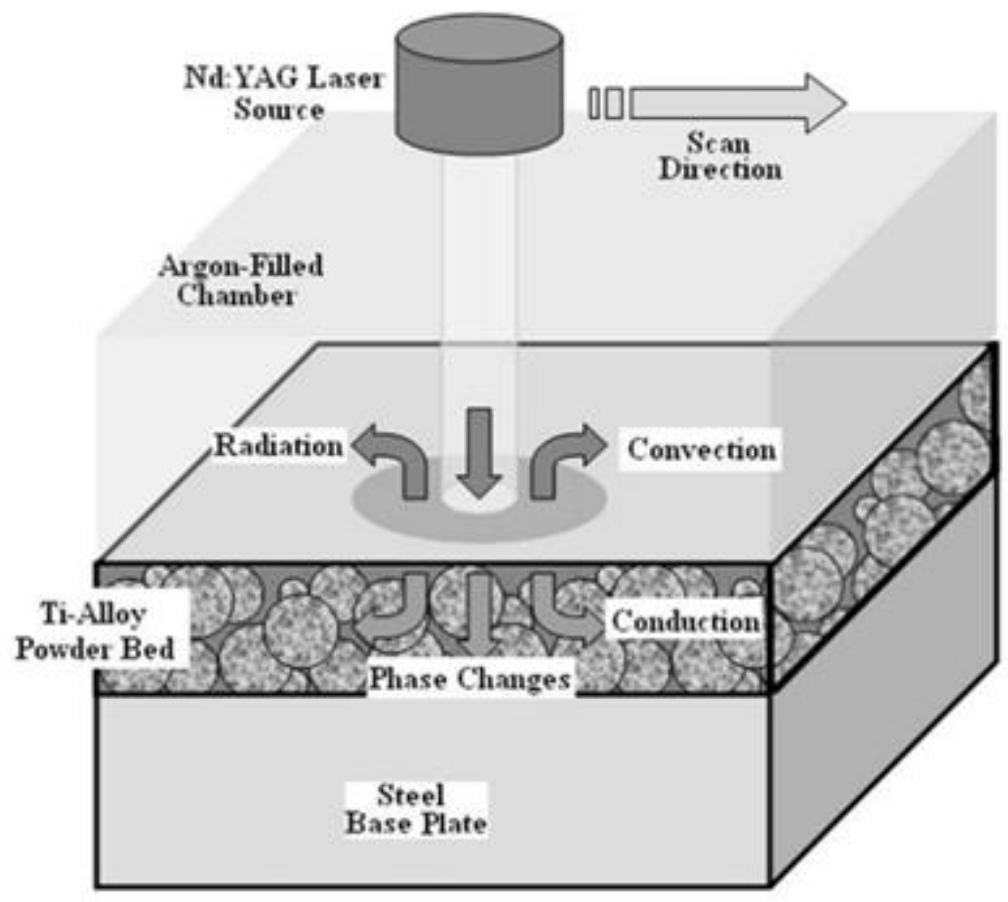

Figure 2 Schematic diagram showing heat transfer phenomena in an SLM process [30] 


\subsubsection{SLM Heat Transfer Phenomenon Description}

Figure 2 is a schematic representation of heat transfer in SLM [30]. The laser scans the top of the powder bed following a prescribed scan pattern. The heat transfer process in SLM consists of different phenomena, including:

- powder bed radiation,

- convection between the powder bed and environment,

- heat conduction inside the powder bed and between the powder bed and substrate,

- latent heat of fusion, and

- heat of vaporization.

The complexity brought about by the powder phase change and the corresponding variation of the thermal properties during SLM also complicates the heat transfer problem.

\subsubsection{SLM Thermal Simulations}

Since temperature distribution in SLM is important, many researchers have put considerable effort toward understanding the SLM process [31-36] and formulating models to describe SLM thermal evolution [37-42]. Simulation models proved beneficial for demonstrating the influence of various input processing parameters. Those models are the essential tools for identifying proper parameters without extensive testing [43].SLM models include a laser source,powder bed held over a base plate, a prescribed scan pattern and relevant the heat transfer mechanism. The latent heat of fusion is large in SLM. The complexity brought about by the powder phase change and the corresponding variation of the thermal properties during SLM also complicate the heat transfer problem. A detailed literature survey describing the heat transfer problem in SLM can be found in [45]. 


\subsection{Ultrasonic Consolidation}

Ultrasonic consolidation (UC) is a solid-state fabrication process that uses additive manufacturing principles to combine ultrasonic metal welding and milling to produce three dimensional freeform objects. The process uses the power of high frequency ultrasonic vibration at low amplitude to bond thin foils of materials to form solid objects. It combines normal and oscillating shear forces on mating foils on the one hand and the resulting friction forces between the materials to fracture and displace surface oxides from the materials. These atomically clean surfaces bond by direct contact under modest pressure and temperatures that are less than half of the melting point of the materials. The materials are thus metallurgically bonded. Fractured oxides and surface impurities in the materials are distributed in the bond zone. The process combines the layer-by-layer addition of foils with contour milling using the integrated 3-axis $\mathrm{CNC}$ machining facilities to produce desired component geometry. It is therefore both an additive and subtractive process. Fabrication involves the generic freeform fabrication process in which a solid CAD model is numerically sliced into thin horizontal layers which are sequentially sent to the fabricating machine to build the part from bottom up.

The UC machine consists of a welding horn, also known as a sonotrode, which exerts normal force and oscillatory high-frequency vibration on the materials to be welded. Welding takes place on a substrate fixed on a heated plate. The UC machine is designed for automatic foil material feed, but materials can also be fed manually. Figure 3 shows the schematic view of the ultrasonic consolidation process. 


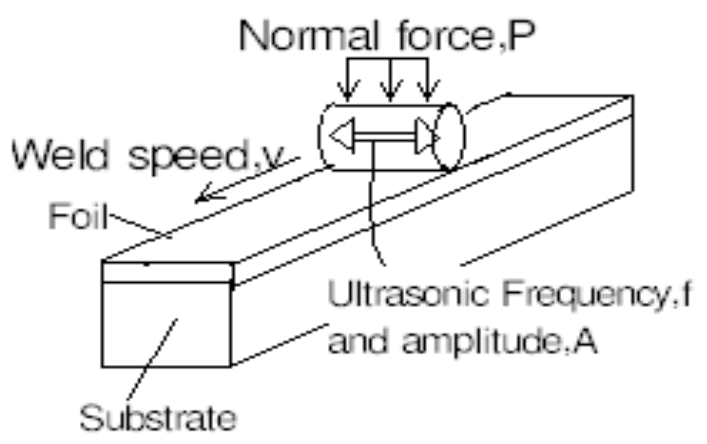

Figure 3 Schematic of UC process [44]

The primary process parameters in UC fabrications are vibration amplitude, temperature, welding speed, and normal force [45]. Other parameters that can affect weld quality include welding sonotrode surface roughness, foil surface finish [46], and how much "overlap" is used between adjacent foils when using the automated material feed system [47]. The optimum process parameters for $\mathrm{Al}$ alloys 3003 and 6061; stainless steel 316L; and $\mathrm{Al} / \mathrm{SiC}$ metal matrix composite have been experimentally determined in earlier work [48-56].

\subsection{Computational Methods}

Computational methods to solve partial differential equations have been classified into two main categories i.e. analytical and numerical solution techniques. Analytical solutions have been limited to simple geometries and material variations with energy conserving systems. A class of techniques such as Green's Function has both analytical and numerical components involved in analysis. Compared to these techniques Finite Element has been used more frequently due to its applicability for cases with complicated geometries and energy dissipative nonlinearities. 
The finite element method (FEM) has been a widely used numerical method for solving systems of partial differential equations (PDEs). A typical FEM algorithm involves discretizing a domain into a finite number of elements and formulating a weak form of the PDE. A weak form of differential equations can be decomposed into a system of linear equations with the help of weighted residuals and the principle of minimum energy. A standard finite element procedure is as follows.

\section{A standard Finite Element algorithm}

1. Domain discretization (breaking structure into small elements)

2. Derivation of a strain operator $[B]$ (linear or nonlinear based on the type of analysis) at the integration point. strain $=[B] q$ where $q$ is the element displacement vector.

3. First variation of element energy with respect to $q$ to determine element stiffness matrix.

4. Assembling the global stiffness matrix.

5. Displacement and traction boundary condition application.

6. Solution of the simultaneous equations generated in the previous steps.

7. Calculation of stress at the integration points.

\subsection{Adaptive Mesh Refinement}

\subsubsection{Introduction}

The computational cost when solving systems of equations derived in FEM and the accuracy of the solution depends on the extent of refinement. A strategy where mesh refinement is decided based on variations across a domain is called adaptive mesh 
refinement (AMR) [57]. AMR is useful for reducing the computational costs involved in problems with significant spatial variations or localized behavior.

\subsubsection{Adaptive Meshing:}

Adaptive mesh refinement was proposed by Berger and Oliger to solve wave equations with localized steep gradients [57-58]. AMR provides significant advantages in terms of reliability and computational costs for simulating systems with different characteristic spatial lengths. For example, wave propagation leads to local deformation which requires

refinement in that region to capture the localized phenomena. The multi-scale nature of AMR enables one to more efficiently solve problems where computational cost and time would be very high if the problem were to be solved with a high resolution mesh everywhere [58]. To apply AMR effectively, acceptable values of error bounds and estimated error are a consideration. The following sections explain different error estimation techniques and their formulation in depth.

\subsubsection{Error Estimates in FEM}

Traditionally, convergence of a finite element model is checked using mesh convergence. This method is computationally expensive for time dependent dynamic problems where adaptive meshing is required. General adaptive meshing software tools [59] employ error estimators to estimate accuracy of the solution obtained within a particular level of refinement. There are two types of error estimators available [60] namely apriori and posteriori estimators. Apriori error estimators are used to understand the asymptotic behavior of the errors but are not used to determine an actual error estimate. Posteriori error estimators work on the solution itself to obtain estimates of the actual 
solution errors. In the following sections both of these estimators are discussed in greater details.

\subsubsection{A Priori Error Estimates}

A priori error estimates [61] provide useful information on the asymptotic behavior of the approximation. The Lax Theorem [62] is the fundamental theorem for apriori error estimation for the numerical solution of PDEs. It starts with a generalized representation of a PDE in the following form where $L$ is a differential operator and $\emptyset$ is an actual solution of the differential equation.

$$
L \emptyset=f
$$

To understand the Lax Theorem assume a system where:

$$
L \varphi=\frac{\partial^{2} \emptyset}{\partial x^{2}}=f
$$

Taylor's expansion of the above equation using central difference approximation gives

$$
\frac{\partial^{2} \emptyset}{\partial x^{2}}=\frac{\emptyset(i+1) h-2 \emptyset(i h)+\emptyset(i-1) h}{h^{2}}+O\left(h^{2}\right)
$$

Assuming a discrete solution

$$
\varphi_{i}=\emptyset(i h)
$$

A discrete operator $L^{h}$ is defined as

$$
L^{h} \varphi=\frac{\varphi_{i+1}-2 \varphi_{i}+\varphi_{i-1}}{h^{2}}
$$


The final discretized system of equations will be

$$
\left(L^{h} \varphi\right)_{i}=f_{i}
$$

$$
\text { where } f_{i}=f(u=i h)
$$

Consistency equations can be further defined as

$$
\begin{gathered}
\emptyset_{i}=\emptyset(i h) \\
\left(L^{h} \varphi\right)_{i}-f_{i}=\tau_{i}=O\left(h^{2}\right)
\end{gathered}
$$

and the stability equation is bounded and independent of $h$.

$$
\left\|\left(L^{h^{-1}}\right)\right\|
$$

Thus error is defined as

$$
\begin{gathered}
\varepsilon=\emptyset-\varphi \\
L^{h} \varepsilon=\tau
\end{gathered}
$$

Convergence is then derived from stability and consistency

$$
\|\varepsilon\| \leq\left\|\left(L^{h^{-1}}\right) \tau\right\| \leq C\|\tau\|
$$

This theorem proves that the convergence is proportional to $h^{2}$ for the elliptic differential equation considered here. This is true with respect to linear FEM formulations with bilinear shape functions. 


\subsubsection{Posteriori Error Estimates}

In FEM, a domain is decomposed into small elements and a solution of the differential equation in each element is assumed to have the form

$$
\widehat{\emptyset}=N_{i}(\overrightarrow{\boldsymbol{x}}) q_{i}
$$

where $N_{i}$ are the shape functions and $q_{i}$ is an approximate solution of the differential equation at the $i^{\text {th }}$ node of the element. A posteriori error [63] in the approximate solution $\widehat{\emptyset}$ is defined as:

$$
e_{\varnothing}(x)=\emptyset(x)-\widehat{\emptyset}(x)
$$

Similarly, errors in integration point variables namely stress $(\sigma)$ and strain $(\varepsilon)$ are defined as:

$$
\begin{aligned}
& e_{\sigma}(x)=\sigma(x)-\hat{\sigma}(x) \\
& e_{\varepsilon}(x)=\varepsilon(x)-\hat{\varepsilon}(x)
\end{aligned}
$$

These error measures are a function of position vector $\vec{x}$ and can be positive or negative depending on the location of $\vec{x}$. A better error indicator should be a scalar so that error criteria can be checked and decisions can be made accordingly. For this purpose, different error norms are used. For example an $L_{2}$ norm is defined as

$$
\|\varnothing\|^{2}=\int_{\Omega} \emptyset(x) \emptyset(x) d \Omega
$$

The energy norm $L_{2}$ of the conjugate stress and strain spaces is one of the most widely applied norms in FEM error analysis of linear elastic structural problems. It is defined as 


$$
\begin{aligned}
\|\varnothing\|^{2}= & \int_{\Omega}(\varepsilon-\stackrel{\mathfrak{m}}{\varepsilon}) E(\varepsilon-\stackrel{\mathfrak{\varepsilon}}{\varepsilon}) d \Omega \\
= & \int_{\Omega} e_{\varepsilon}^{T} e_{\sigma} E d \Omega
\end{aligned}
$$

A relative percentage error norm is defined as

$$
n=100 \times \frac{\text { norm of the error }(e)}{\text { norm of the corresponding variable } \emptyset}
$$

The main challenge in the posteriori error estimate mentioned so far is to derive an exact solution for the differential equation $(\varnothing(x))$. It is not possible in most practical problems involving complex geometries to find an exact analytical solution. It has been shown that in continuous smoothened solutions for stress and strain it is more accurate compared to piecewise approximate solutions determined using FEM. Among various methods of smoothening FEM solutions, Super Convergent Patch (SCP) recovery [64] of the elements is one of the most accurate methods.

\subsubsection{Super Convergent Patch Recovery}
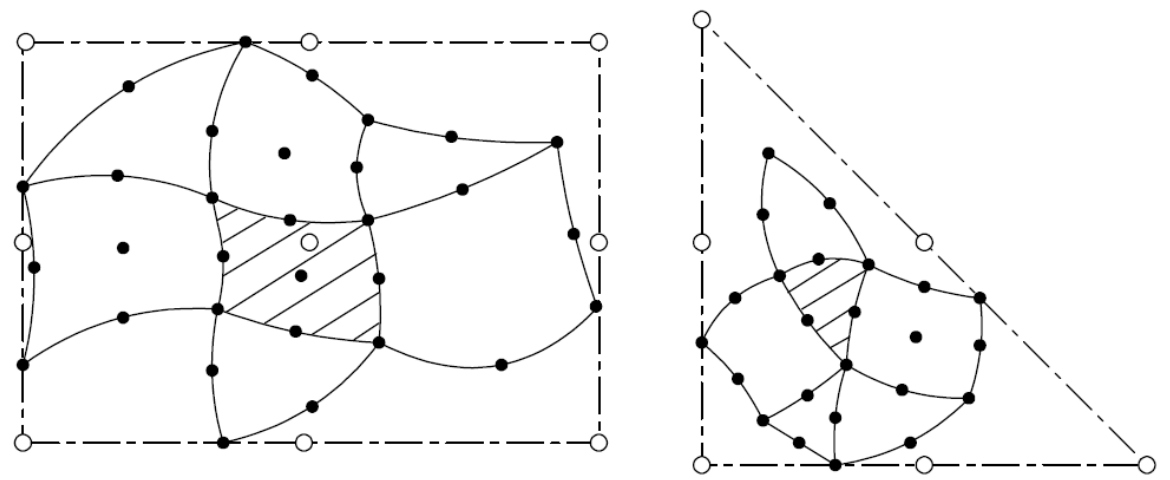

Figure 4 Constant Jacobian Patch [63]

It has been shown that among various smoothening techniques SCP gives the most accurate smoothened solution with respect to an analytical solution. The accuracy of 
recovery makes it an ideal candidate for error estimation. It can be used with nodal variable as well as Gauss Point variables which are functions of first or higher derivatives of the nodal variable. Considering type of variable, SCP can be classified into the following types:

- Node based patch: Adjacent elements in a group around a particular node of interest

- Element Based: All elements adjacent to a particular element of interest

- Face based patch: All elements sharing some common face with a particular element of interest

It is recommended that each element should use its own patch to recover a variable in its domain. Figure 4 shows an element based constant Jacobian patch.

SCP recovery is not a fixed process but it is more like a heuristic. It requires creating a database of points of interest in a patch attached to each element. In case of a nodal variable these interest points will be nodes inside a patch and for Gauss Point variables it will be Gauss Points of elements selected inside the patch. Once such a database of nodes and corresponding variable values are prepared, smoothening functions are calculated using least square interpolation based on the geometry of the patch and accuracy required. Generally polynomial or serendipity polynomial functions are used for this purpose. Unknowns involved in these polynomials are determined using least square fit for the information available at nodes in the database.

\subsubsection{Classification of Adaptive Meshes}

Based on the type of PDEs, complexity of the geometry, and the boundary conditions, different AMR strategies are used accordingly. These strategies can be classified into 
structured and unstructured mesh strategies based on the geometrical patterns of the elements and nodes.

\subsubsection{Structured Meshes}

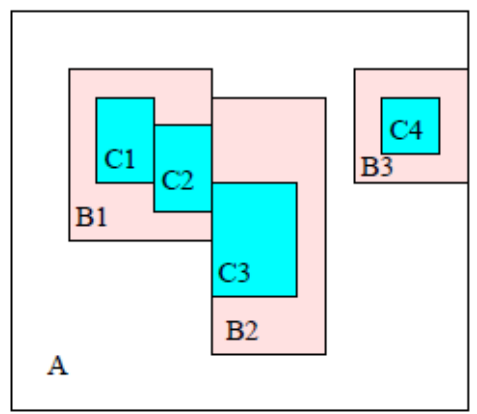

(a)

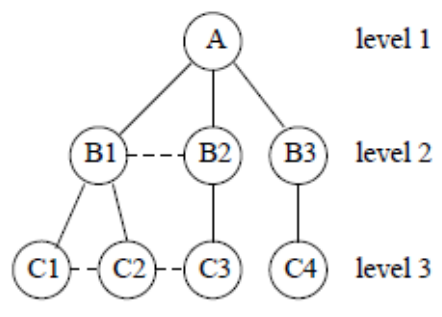

(b)

Figure 5 (a) Geometry and (b) data structure of a 3-level mesh tree. [84]

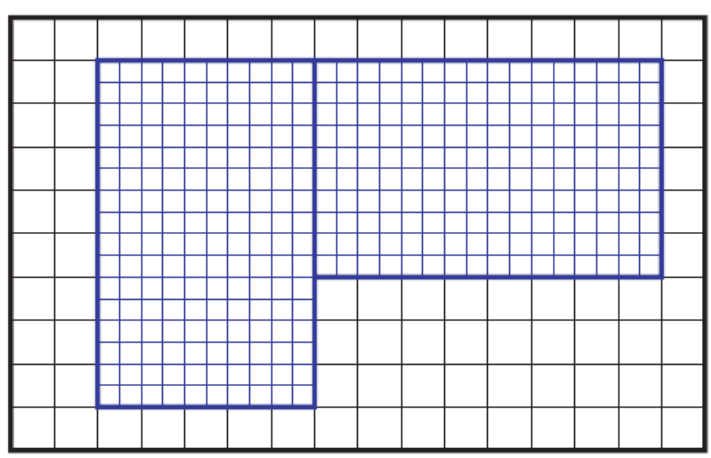

Figure 6 Mesh strategy for block structured refinement [67]

In structured adaptive meshes [65], the orthonormality of the grid is strictly maintained. The basis of the mesh can be regular Cartesian or curvilinear in nature. Block structured mesh refinement involves a mesh refinement of multiple sub domains such that two domains can share a boundary but no area can be shared. Each domain can be meshed independently and can be further refined to reach higher amounts of resolution. This strategy can be explained through a tree diagram as shown in figure 5. The refinement 
inside each subdomain creates a mismatch of mesh density at the interface between the coarse grid and the refined subdomain grid and can lead to erroneous results. This can be avoided with additional constraints at interfaces. The disadvantage of having additional constraints is that the solution may not reach its equilibrium distribution.

The irregular nodes in a block structured adaptive mesh as shown in Figure 6 can be avoided with an adaptive mesh generated with mesh distortion as shown in figure 7 . This method is relatively easy to implement but the unnecessary refinement of elements above and below the main refinement region along with bad aspect ratios in nearby regions restricts its usage compared to block structured mesh refinement.

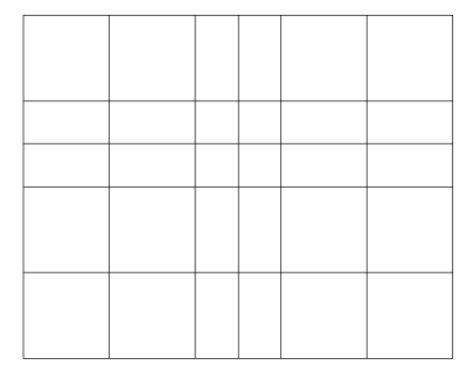

Figure 7 Adaptive Mesh with Mesh Distortion [65]

\subsubsection{Unstructured Meshes}

Triangulation is an easier way to discretize complicated geometries and for a few geometries it is the only method of discretization. Availability of automated triangulation algorithms such as Delaunay triangulation [68] makes it easy to implement for any general geometry. However adaptive meshing with triangulation poses unique challenges. In the 
following sub-sections, widely applied refinement and de-refinement methods used for unstructured triangular meshes are briefly discussed.

Bank's Uniform Bisection Method:

Bank [69] developed Piecewise Linear Triangular Multi-grid Finite Element Program software (PLTMG) for adaptive mesh refinement with unstructured meshes for solution of Elliptic Partial Differential Equations. The Refinement is achieved by uniform bisection of a triangular element into four small elements as shown in figure 8 . This will lead to three irregular nodes on all three sides of the parent triangle. To avoid these irregular nodes he incorporated new triangular elements into the neighboring parent triangular elements. These subdivisions including parent triangle subdivisions are shown with dotted lines.

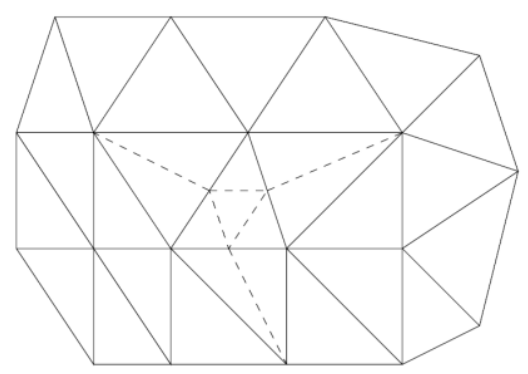

Figure 8 Bank's uniform subdivision in first level refinement [65]

Further refinement of the triangulation in the Bank's scheme is done with uniform subdivision of new triangles as shown in figure 9. Such subsequent subdivisions will avoid further subdivision of the resulting new triangles with bad shape. 


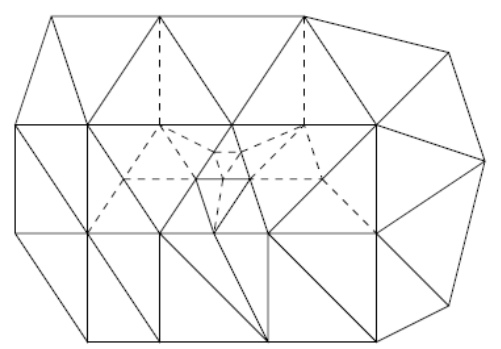

Figure 9 Second Level refinement in Bank's method [65]

\section{Riveras's Bisection Method}

Rivera [65] proved that iterative bisection of the longest side of a triangle will lead to a regular mesh without any irregular nodes. The first two iterations of this procedure are shown in figure 10. Further he showed that the lower bound of the smallest angle produced in this scheme will be equal to half of the smallest angle of the original triangulation. The algorithm used by Rivera is shown in Table 1 .
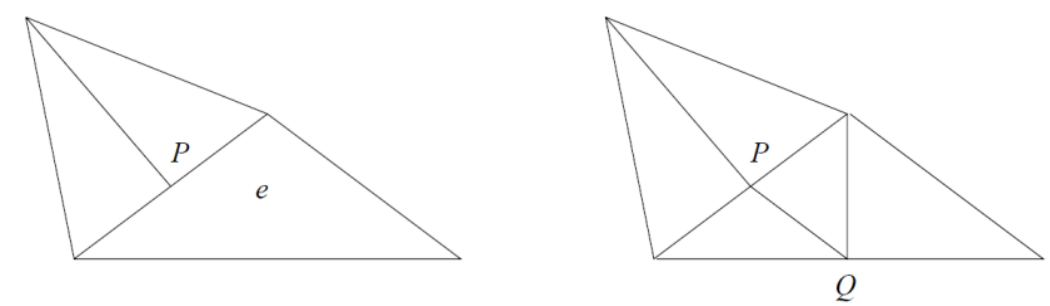

Figure 10 First two iterations of Rivera's Bisection of the longest side of a triangle [65]

De-refinement of Unstructured Meshes

Banks's and Rivera's refinement methods can be represented using tree structures. The same tree structure can be used for de-refinement purposes. The offspring elements at a particular level of a tree structure can be pruned to establish a higher order coarse mesh. Coarsening done without a tree structure can be achieved by edge collapsing as shown in 
figure 11. In [70] it has been shown that for a $2 \mathrm{D}$ problem, $V_{0}$ is collapsed into $V_{1}$, and $V_{1}, V_{2}, V_{3}, \ldots, V_{k}$ are the nodes of the elements connected with node $V_{0}$. The node $V_{0}$ is called the collapsing vertex and $V_{1}$ is called the target vertex. Collapsing a vertex needs a prior check on the geometry of the original mesh. An example where collapsing can lead to an incorrect mesh with a negative vertex angle at one triangle is shown in figure 12 .

Table 1: Rivera's Algorithm

Rivera's Algorithm [65]:

Obtain $\Delta_{h}^{1}$ by bisecting all triangles of $\delta$ by their longest edges

Let $\rho^{1}$ contain those elements of $\Delta_{h}^{1}$ having irregular nodes

$\mathrm{i}:=1$

while $\rho^{i} \neq \varnothing$ do

Let $e \in \rho^{i}$ have an irregular node $P$ and bisect $e$ by its longest edge

Let $\mathrm{Q}$ be the intersection point of this bisection

if $P \neq Q$ then

Join $P$ and $Q$

end if

Let $\Delta_{h}^{i+1}$ be the mesh created by this process

Let $\rho^{i+1}$ be the set of elements in $\Delta_{h}^{i+1}$ with irregular nodes

$\mathrm{i}:=\mathrm{i}+1$

end while

$\operatorname{return} \Delta_{h}^{i}$ 

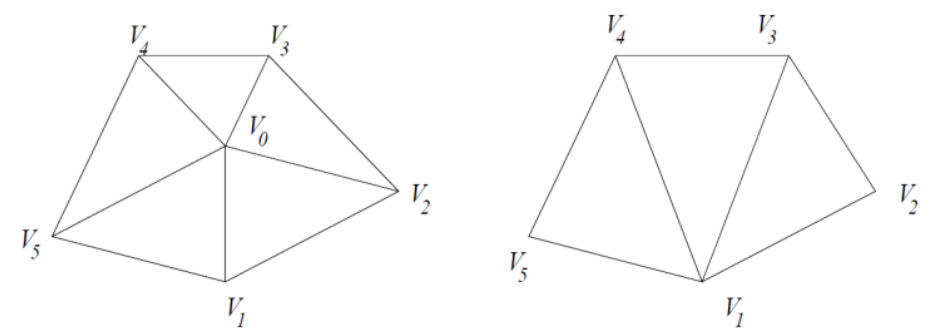

Figure 11 Coarsening of a triangulation by Edge Collapsing [65]
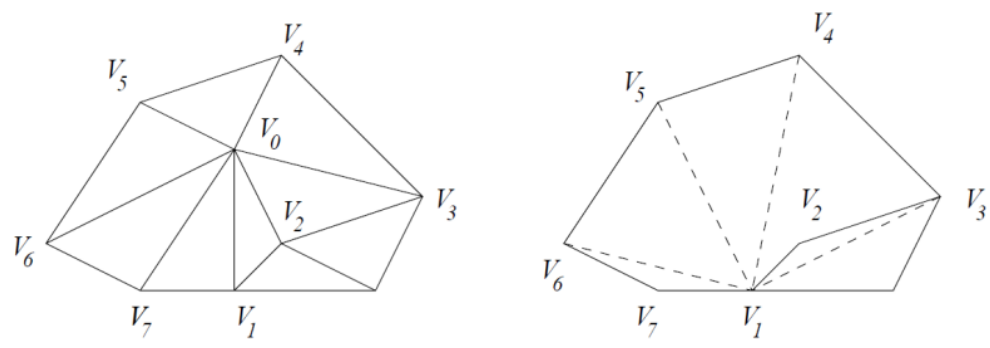

Figure 12 Example triangulation where collapsing a vertex can lead to an erroneous mesh

\subsubsection{Data transfer between non-matching meshes with matching domain}

Certain classes of problems such as large deformation or time dependent dynamic problems require adaptive dynamic mesh refinement where the mesh is dynamically adapted to new boundary conditions, evolving constitutive laws or geometry. This dynamic adaptivity of the mesh calls for data transfer from one mesh to the newly adapted mesh [71-72]. Various interpolation techniques are used for this purpose. In the following discussion two widely used interpolation techniques [72] namely the Radial Basis Function Method and the Inverse isoparametric method are discussed as examples of interpolation from one mesh to another where the boundary and domain of the two meshes match perfectly. 


\subsubsection{Radial basis function method:}

The radial basis function method has been found to produce high quality solutions to multivariate scattered data interpolation problems. Originally a part of the spline method [71], this method was proposed by Wendland [73] and Beckert [74] as being suitable for dealing with interpolation of variables between FEM meshes. If $s(x)$ is the actual function and has values $f_{i}=\left(f_{1}, f_{2}, f_{3}, \ldots, f_{N}\right)$ at points $\left(x_{1}, x_{2}, x_{3}, \ldots, x_{N}\right)$, then by assuming the radial basis approximation function as $s(x)$, we have

$$
s\left(x_{i}\right)=f_{i}
$$

This means that the radial basis interpolation function will provide the exact same value for input data points for which interpolation has been performed. Further the radial basis function has the following form.

$$
s(x)=p(x)+\sum_{i=1}^{N} \lambda_{i} \emptyset\left(\left\|x-x_{i}\right\|\right)
$$

where $\emptyset$ is a radially decaying function of radial Euclidian distance $\left\|x-x_{i}\right\|$ and $p(x)$ is a polynomial function. It can be seen from the above functional form that the radial basis function is the sum of radial symmetric functions for each data point with weight $\lambda_{i}$. Further, it is improved by adding a polynomial function. According to [75] a naive approach to the solution of interpolation equation (18) leads to a complexity of $\mathrm{O}\left(N^{3}\right)$ in time and $\mathrm{O}\left(N^{2}\right)$ in space where $N$ is the number of nodes. Due to the involved computational complexity, it is not suitable for moderate to large size problems compared to the inverse isoparametric method which is discussed hereafter. 


\subsubsection{Inverse Isoparametric Mapping Method}

Inverse isoparametric mapping is a map where an element is mapped from a global coordinate system to an isotropic coordinate system. Figure 13 shows a $2 \mathrm{D}$ four node element in the global coordinate system and the same element mapped into isoparametric coordinates. The advantage of using an isoparametric system in FEM simulations is that it removes the need for calculating the coefficients of shape functions which are required in the case of global coordinate systems.

Isoparametric mapping has the additional capability of interpolating global coordinates along with the nodal variables with the same shape function. Shape functions for the element considered in this discussion are:

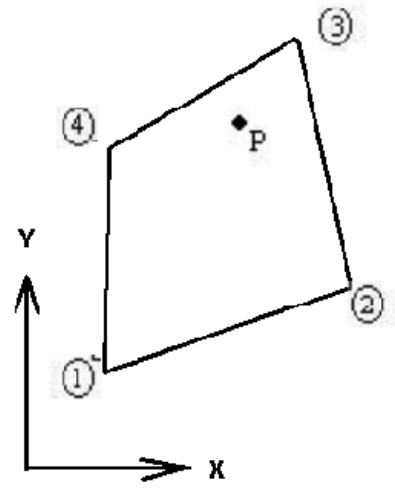

Global Coordinate System

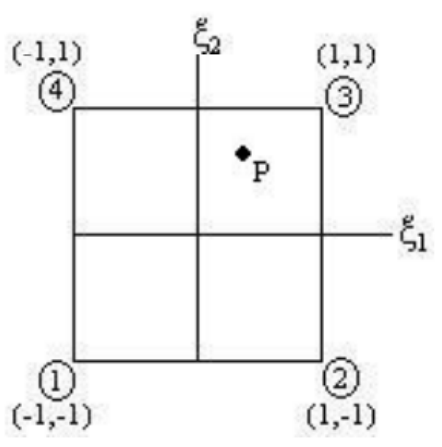

Isoparametric System

Figure 13 Global Coordinate System and Isoparametric Element

$$
\begin{aligned}
& N_{1}=\frac{1}{4}\left(1-\zeta_{1}\right)\left(1-\zeta_{2}\right) \\
& N_{2}=\frac{1}{4}\left(1+\zeta_{1}\right)\left(1-\zeta_{2}\right)
\end{aligned}
$$




$$
\begin{aligned}
& N_{3}=\frac{1}{4}\left(1+\zeta_{1}\right)\left(1+\zeta_{2}\right) \\
& N_{4}=\frac{1}{4}\left(1-\zeta_{1}\right)\left(1+\zeta_{2}\right)
\end{aligned}
$$

Using these shape functions, global coordinates and the variable $\varnothing$ will be mapped as follows where $x_{i}$ and $y_{i}$ are element node coordinates of the $i^{\text {th }}$ point and $\emptyset_{i}$ is the value of the variable at the $i^{\text {th }}$ node.

$$
\begin{aligned}
& x=\sum_{i=1}^{4} N_{i}(\zeta) x_{i} \\
& y=\sum_{i=1}^{4} N_{i}(\zeta) y_{i} \\
& \widehat{\emptyset}=\sum_{i=1}^{4} N_{i}(\zeta) \emptyset_{i}
\end{aligned}
$$

Given the isoparametric solution, global coordinates as well as $\widehat{\varnothing}$ can be obtained. For the interpolation of $\varnothing$ on new mesh nodes, the $\zeta$ coordinates of the new mesh points are required to be known apriori. This calls for solving the following equations derived from the above-mentioned equation for $\zeta$ [76].

$$
\begin{gathered}
x_{p}=a_{1}+a_{2} \zeta_{1}+a_{3} \zeta_{2}+a_{4} \zeta_{1} \zeta_{2} \\
y_{p}=b_{1}+b_{2} \zeta_{1}+b_{3} \zeta_{2}+b_{4} \zeta_{1} \zeta_{2} \\
\text { where } \\
a_{1}=\frac{1}{4}\left(x_{1}+x_{2}+x_{3}+x_{4}\right)
\end{gathered}
$$




$$
\begin{gathered}
a_{2}=\frac{1}{4}\left(-x_{1}+x_{2}+x_{3}-x_{4}\right) \\
a_{3}=\frac{1}{4}\left(-x_{1}-x_{2}+x_{3}+x_{4}\right) \\
a_{4}=\frac{1}{4}\left(-x_{1}+x_{2}-x_{3}+x_{4}\right) \\
b_{2}=\frac{1}{4}\left(-y_{1}+y_{2}+y_{3}-y_{4}\right) \\
b_{3}=\frac{1}{4}\left(-y_{1}-y_{2}+y_{3}+y_{4}\right) \\
b_{4}=\frac{1}{4}\left(-y_{1}+y_{2}-y_{3}+y_{4}\right)
\end{gathered}
$$

Simple substitutions can lead to a quadratic equation for solving $\zeta_{1}$ :

$$
A\left(\zeta^{1}\right)^{2}+B \zeta^{1}+C=0
$$

Where

$$
\begin{gathered}
\mathrm{A}=\mathrm{a}_{2} \mathrm{~b}_{4}-\mathrm{a}_{4} \mathrm{~b}_{2} \\
\mathrm{~B}=\mathrm{a}_{1} \mathrm{~b}_{4}+\mathrm{a}_{2} \mathrm{~b}_{3}-\mathrm{a}_{3} \mathrm{~b}_{2}-\mathrm{a}_{4} \mathrm{~b}_{1}+\mathrm{a}_{4} \mathrm{y}_{\mathrm{p}}-\mathrm{b}_{4} \mathrm{x}_{\mathrm{p}} \\
C=a_{1} b_{3}-a_{3} b_{1}+a_{3} y_{p}-b_{3} x_{p}
\end{gathered}
$$

$\zeta_{2}$ can be obtained by substituting $\zeta_{1}$ into one of the main equations. The advantage of this method is that it interpolates variables according to the basic assumptions of FEM and computationally it is less expensive compared to other interpolation methods. One of the 
main limitations of this method in the case of $3 \mathrm{D}$ formulation is that it needs a numerical solution for a system of three nonlinear equations.

\subsection{Spatial Homogenization:}

In classical mathematical homogenization theory [77], displacements are expanded as

$$
u_{i}^{\zeta}(X, t)=u_{i}^{0}(X, t)+\zeta u_{i}^{1}(X, Y, t)+\zeta^{2} u_{i}^{2}(X, Y, t)+o\left(\zeta^{3}\right)
$$

Where $\mathbf{X}$ and $\mathbf{Y}$ are defined as shown in figure 14. $\mathbf{Y}$ represents the material coordinate in the initial microscopic (unit cell) domain and $\mathbf{y}$ is the deformed coordinate in the unit cell domain. The two macro and micro scale coordinates are related by $\mathrm{Y} \equiv \mathrm{X} / \zeta$ with $0<\zeta \ll$ 1.

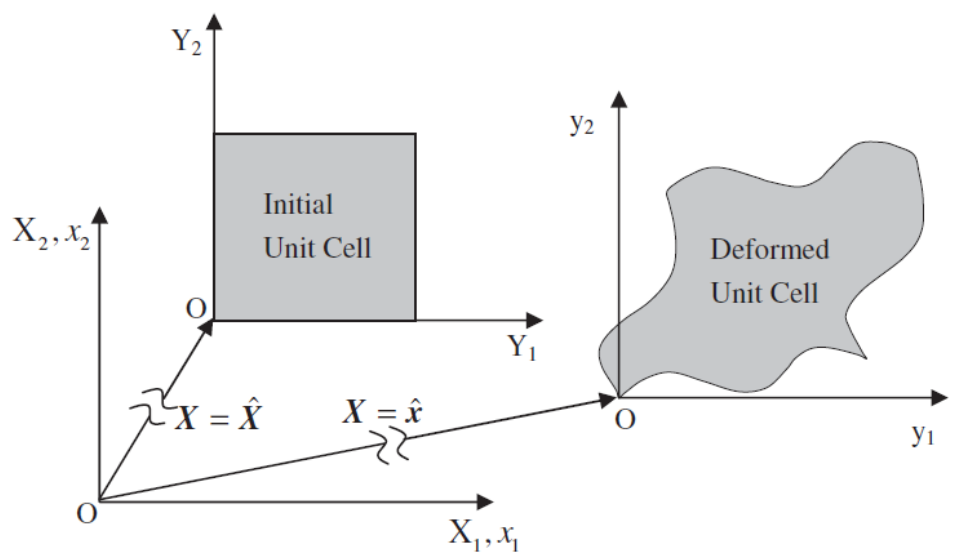

Figure 14 Macro and micro coordinate systems. [77]

Assumptions in this formulation are as following.

1. Two scales are separable.

2. Maximum displacement magnitudes of successive lower scales follow asymptotic convergence. 
3. Size of unit cell is much smaller compared to the macroscopic dimension of the body.

Based on the above assumptions, the overall (macro) deformation gradient is obtained from differentiation of the leading term in the asymptotic expansion as following.

$$
\overline{F_{i k}}(X, t)=\frac{1}{\left|\Theta_{Y}\right|} \int_{\Theta_{Y}} F_{i k}(X, Y, t) d \Theta_{Y}=\left[\delta_{i k}+\frac{\partial u_{i}^{0}(X, t)}{\partial X_{k}}\right]_{a t X=\widehat{X}}
$$

This equation assumes that the contribution of $\mathrm{u}_{\mathrm{i}}^{1}$ to the macroscopic deformation gradient is negligible. This leads to the periodic boundary conditions shown in figure 15. This can be explained mathematically as following.

$$
u_{i}^{1}(X, Y, t)=u_{i}^{1}(X, Y+k L, t)
$$

Where $\mathrm{L}$ is the characteristic size of the cell.

(a)

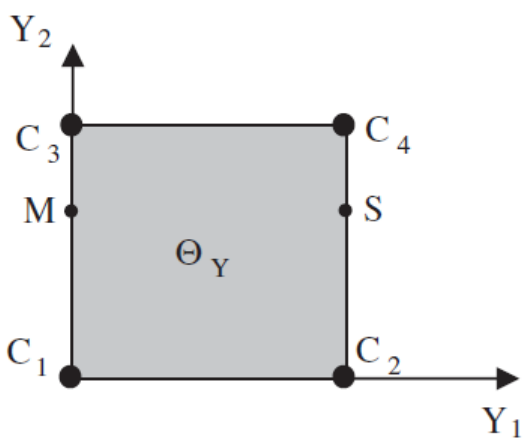

(b)

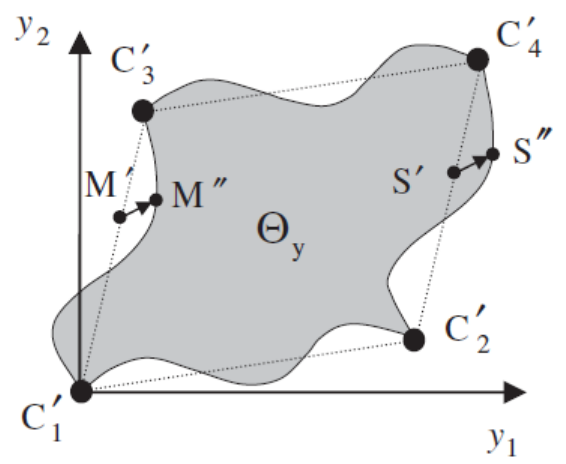

Figure 15 Definition of periodic boundary conditions: (a) initial unit cell and (b) deformed unit cell. [77] 


\title{
CHAPTER 4
}

\section{A GENERALIZED FEED FORWARD DYNAMIC}

\author{
ADAPTIVE MESH REFINEMENT AND DE-
}

REFINEMENT FINITE ELEMENT FRAMEWORK FOR

SPATIOTEMPORALLY PERIODIC LOCALIZED

\section{BOUNDARY CONDITIONS: APPLICATION TO METAL}

\section{LASER SINTERING}

\subsection{Introduction}

The finite element method (FEM) is a widely used numerical method for solving systems of partial differential equations (PDEs). A typical FEM algorithm involves discretizing a domain into a finite number of elements and formulating a weak form of the PDE. A weak form of differential equations can be decomposed into a system of linear equations with the help of weighted residuals or the principle of minimum energy. The computational cost when solving these systems of equations and the accuracy of the solution depends on the extent of refinement. A strategy where mesh refinement is decided based on variations across a domain is called adaptive mesh refinement (AMR)[78]. AMR 
is useful for reducing the computational costs involved in problems with significant spatial variations or localized behavior.

\subsubsection{Adaptive Meshing:}

Adaptive mesh refinement was proposed by Berger and Oliger to solve wave equations with localized steep gradients [78-79]. AMR provides significant advantages in terms of reliability and computational costs for simulating systems with different characteristic spatial lengths. For example, wave propagation leads to local deformation which requires refinement in that region to capture the localized phenomena. The multi-scale nature of AMR enables one to more efficiently solve problems where computational cost and time would be very high if the problem were to be solved with a high resolution everywhere [79].

To apply AMR effectively, acceptable values of error bounds and estimated error are a consideration. The following subsection explains different error estimation techniques and their formulation in depth.

\subsubsection{Error Estimates in FEM}

Traditionally, convergence of a finite element model is checked using mesh convergence. This method is computationally expensive for time dependent dynamic problems where adaptive meshing is required. General adaptive meshing software tools [80] employ error estimators to estimate accuracy of the solution obtained within a particular level of refinement. There are two types of error estimators available [81] namely apriori and posteriori estimators. Apriori error estimators are used to understand the asymptotic behavior of the errors but are not used to determine an actual error estimate. 
Posteriori error estimators work on the solution itself to obtain estimates of the actual solution errors. In the following sections both of these estimators are discussed in greater details.

\subsubsection{A Priori Error Estimates}

A priori error estimates provide useful information on the asymptotic behavior of the approximation. The Lax Theorem [82] is the fundamental theorem for apriori error estimation in the numerical solution of PDEs. It starts with a generalized representation of

a PDE in the following form where $L$ is a differential operator and $\emptyset$ is an actual solution of the differential equation.

$$
L \emptyset=f
$$

to understand the Lax Theorem assume a system where:

$$
L \varphi=\frac{\partial^{2} \emptyset}{\partial x^{2}}=f
$$

Taylor's expansion of the above equation using central difference approximation gives

$$
\frac{\partial^{2} \emptyset}{\partial x^{2}}=\frac{\emptyset(i+1) h-2 \emptyset(i h)+\emptyset(i-1) h}{h^{2}}+O\left(h^{2}\right)
$$

Assuming a discrete solution

$$
\varphi_{i}=\emptyset(i h)
$$

A discrete operator $L^{h}$ is defined as 


$$
L^{h} \varphi=\frac{\varphi_{i+1}-2 \varphi_{i}+\varphi_{i-1}}{h^{2}}
$$

The final discretized system of equations will be

$$
\left(L^{h} \varphi\right)_{i}=f_{i}
$$

where $f_{i}=f(u=i h)$

Consistency equations can be further defined as

$$
\begin{gathered}
\emptyset_{i}=\varnothing(i h) \\
\left(L^{h} \varphi\right)_{i}-f_{i}=\tau_{i}=O\left(h^{2}\right)
\end{gathered}
$$

and the stability equation

$$
\left\|\left(L^{h^{-1}}\right)\right\|
$$

is bounded and independent of $h$.

Thus error is defined as

$$
\begin{gathered}
\varepsilon=\emptyset-\varphi \\
L^{h} \varepsilon=\tau
\end{gathered}
$$

Convergence is then derived from stability and consistency

$$
\|\varepsilon\| \leq\left\|\left(L^{h^{-1}}\right) \tau\right\| \leq C\|\tau\|
$$


This theorem proves that the convergence is proportional to $h^{2}$ for the elliptic differential equation considered here. This is true with respect to linear FEM formulations with bilinear shape functions.

\subsubsection{Posteriori Error Estimates}

In FEM, a domain is decomposed into small elements and a solution of the differential equation in each element is assumed to have the form

$$
\widehat{\emptyset}=N_{i}(\overrightarrow{\boldsymbol{x}}) q_{i}
$$

where $N_{i}$ are the shape functions and $q_{i}$ is an approximate solution of the differential equation at the $i^{\text {th }}$ node of the element. A posteriori error [83] in the approximate solution $\widehat{\emptyset}$ is defined as:

$$
e_{\emptyset}(x)=\emptyset(x)-\widehat{\emptyset}(x)
$$

Similarly, errors in integration point variables namely stress $(\sigma)$ and strain $(\varepsilon)$ are defined as:

$$
\begin{aligned}
& e_{\sigma}(x)=\sigma(x)-\hat{\sigma}(x) \\
& e_{\varepsilon}(x)=\varepsilon(x)-\hat{\varepsilon}(x)
\end{aligned}
$$

These error measures are a function of position vector $\vec{x}$ and can be positive or negative depending on the location of $\vec{x}$. A better error indicator should be a scalar so that error criteria can be checked and decisions can be made accordingly. For this purpose, different error norms are used. For example an $L_{2}$ norm is defined as 


$$
\|\varnothing\|^{2}=\int_{\Omega} \emptyset(x) \emptyset(x) d \Omega
$$

The energy norm $L_{2}$ norm of the conjugate stress and strain spaces is one of the most widely applied norms in FEM error analysis of linear elastic structural problems. It is defined as

$$
\begin{aligned}
& \|\emptyset\|^{2}=\int_{\Omega}(\varepsilon-\tilde{\xi}) E(\varepsilon-\stackrel{\widetilde{\varepsilon}}{\tilde{\varepsilon}}) d \Omega \\
& =\int_{\Omega} e_{\varepsilon}^{T} e_{\sigma} E d \Omega
\end{aligned}
$$

A relative percentage error norm is defined as

$$
n=100 \times \frac{\text { norm of the error }(e)}{\text { norm of the corresponding variabe } \emptyset}
$$

The main challenge in the posteriori error estimate mentioned so far is to derive an exact solution $\emptyset(x)$ for the differential equation. It is not possible in most practical problems involving complex geometries to find an exact analytical solution. It has been shown that in continuous smoothened solutions for stress and strain it is more accurate compared to piecewise approximate solutions determined using FEM. Among various methods of smoothening FEM solutions, Super Convergent Patch (SCP) recovery [84] of the elements is one of the most accurate methods.

In order to reduce the error peaks due to localized variations and steep gradients in material response, various localized refinement methods have been proposed. The refinements are adaptive in nature where the refinement sizes are corrected inversely with error magnitudes. The process of correction of refinement sizes is iterative in nature where the refinements are corrected until the local error magnitudes are below a user-specified 
error-tolerance. Generally, the error-tolerances are kept as a very small fraction of the initial maximum response magnitude or initial maximum magnitude of the external stimulus or combinations of both depending on the definition of error calculation. When the error is out of the bound, the mesh is refined adaptively to bring it within the tolerance level. Various structured adaptive mesh refinement strategies are discussed in the next sub-section. Unstructured adaptive mesh refinement strategies have not been discussed here since they are not within the scope of the application.

\subsubsection{Structured Adaptive Meshes}

In structured adaptive meshes, the mapped orthonormality of the grid is strictly maintained or a particular motif is maintained. The basis of the mesh can be regular Cartesian or curvilinear in nature. Block structured mesh refinement [78-79] involves a mesh refinement of multiple sub domains such that two domains can share a boundary but no area can be shared. Each domain can be meshed independently and can be further refined to reach higher amounts of resolution. This strategy can be explained through a tree diagram as shown in figure 16 . The refinement inside each subdomain creates a mismatch of mesh density at the interface of the coarse grid and refined subdomain grid (Figure 17) and can lead to erroneous results. This can be avoided with additional constraints at interfaces. The disadvantage of having additional constraints is that the solution may not reach its equilibrium distribution. 


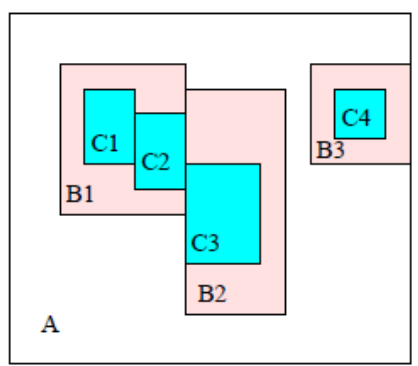

(a)

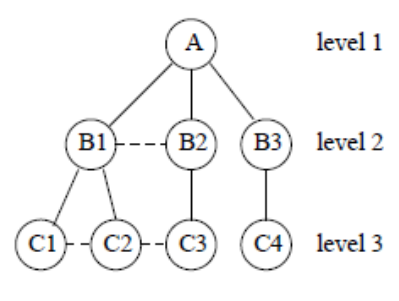

(b)

Figure 16: (a) Geometry and (b) data structure of a 3level mesh tree [84].
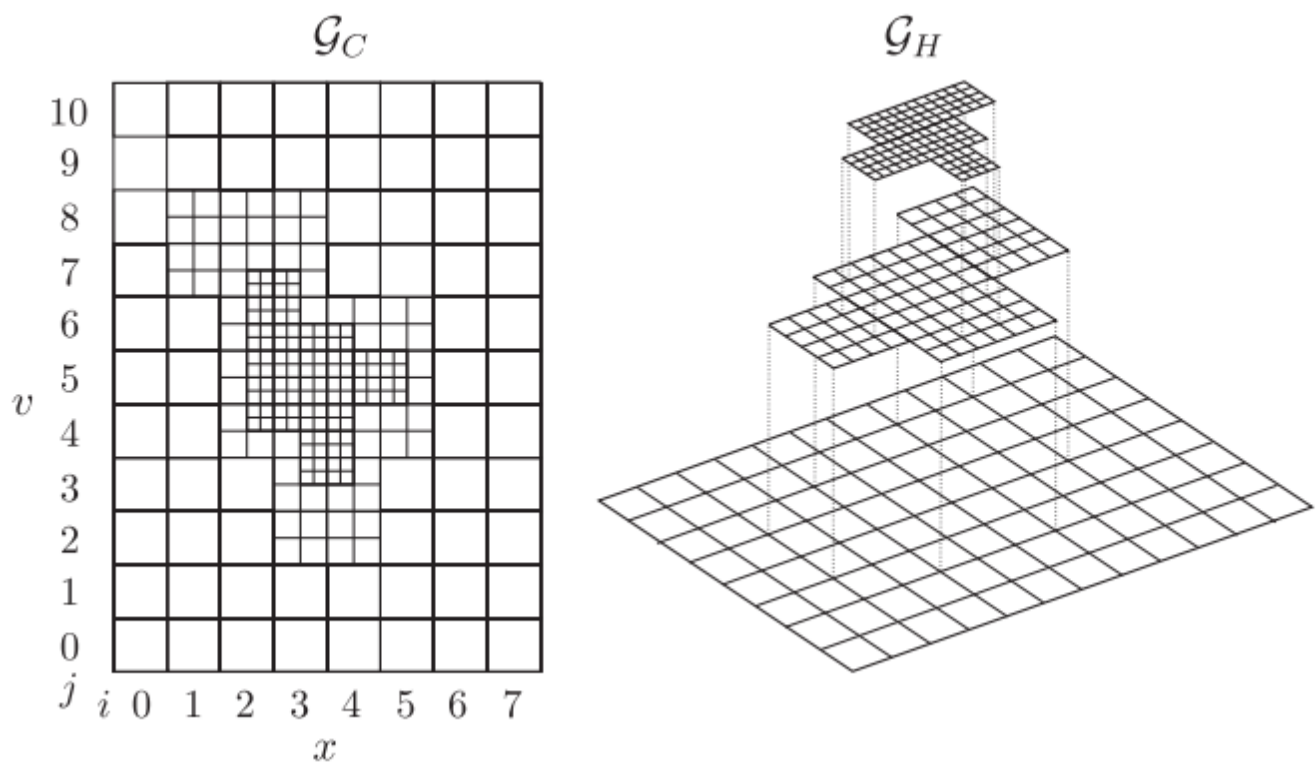

Figure 17 An example of a three-level, block-structured AMR hierarchy [86].

The irregular nodes in the block structured adaptive mesh can be avoided with an adaptive mesh generated with mesh distortion as shown in figure 18. This method has been used along with Multizone Adaptive Grid Generation (MAGG) [87] or Moving Meshes [88]. These methods require additional computations to compute the characteristics of a curvilinear mesh grid as shown in figure 18. 


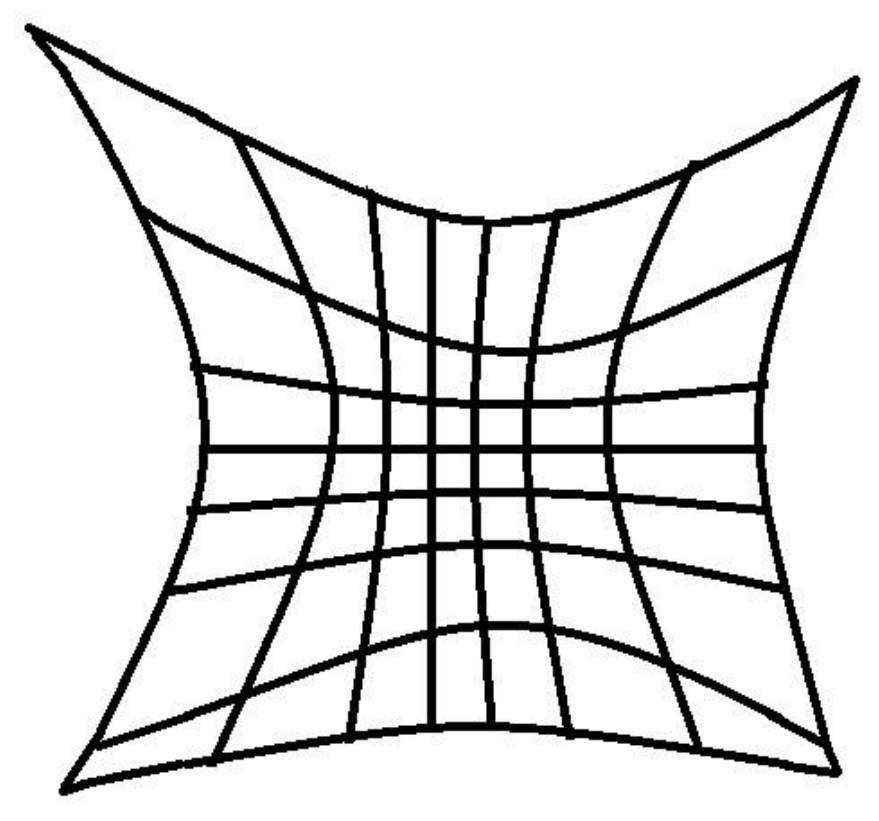

Figure 18 Adaptive Mesh with Mesh Distortion

\subsubsection{Adaptive Meshing in Dynamic Problems and Data Transfer between Meshes}

Certain classes of problems such as large deformation or time dependent dynamic problems require adaptive dynamic mesh refinement where the mesh is dynamically adapted to new boundary conditions, evolving constitutive laws or geometry. This dynamic adaptivity of the mesh calls for data transfer from the parent mesh to the newly adapted mesh [89,90]. Various interpolation techniques are used for this purpose. Two widely used interpolation techniques [89-95] are namely the Radial Basis Function Method and the Inverse Isoparametric Method. In the present work the Isoparametric interpolation method [95] is used considering its computational efficiency and compatibility with FEM discretization assumptions. The next subsection describes a special class of dynamic problems where refinement, de-refinement and data transfer is required frequently. 


\subsubsection{Literature on dynamic adaptive mesh generation}

"Dynamic adaptive meshing" has been used for various types of adaptive meshing strategies which involve meshing adaptively for dynamic boundary conditions or response evolution. A dynamically adaptive mesh [96] has been prepared with the help of a moving mesh strategy involving solution of Variational equations derived for moving mesh generation. Similar moving finite element method algorithms with various error criteria have been tried in the literature. An error based re-distribution [97] and adaptivity in moving mesh has been tried and implemented to solve localized dynamic problems. A level set method [98] has also been used for creating a dynamic moving mesh.

In the case of spatiotemporal problems, a moving mesh requires a large number of adaptations, which entail solving the moving mesh equations multiple times. The block structured refinement can provide the same computational reduction because it does not require solving moving mesh equations to generate the mesh. The disadvantage associated with this methodology is that large number local adaptations are required to follow a moving energy source. The error estimate calculations and graph generation can be avoided with intelligent schemes where patterns learned [99] from similar problems are used to develop intelligent schemes. Combining block structured refinement and intelligent adaptive meshing strategies can help reduce the time required to perform error estimates and matrix assembly up to certain extent but at every iteration stiffness matrices are required to be assembled again.

An easier methodology to solving these problems is to create a refined moving region which is upper bounded on refinement. This will avoid repeated error estimate calculations, 
mesh graph generation and the possibility of intelligent matrix reassembly. A similar moving refinement of the localized regions near a machine tool has been attempted with coded box cell (CBC) [100] excluding a de-refinement of the refined localized region. In the present work a new strategy is introduced to generate a moving finite element refinement zone with a cut-paste-solve-restore algorithm. Mesh graph generation (excludes stiffness matrix assembly) using a cut-paste strategy [101] has been attempted for triangulated meshes. The computational time required to generate stiffness matrices for a newly adapted mesh graph is significantly higher compared to the new mesh graph generation or modification. This work will also focus on efficient regeneration of the stiffness matrix for the new location of the moving refinement box.

\subsubsection{Literature on FEM Simulations in Metal Laser Sintering}

A two dimensional plain stress model [102] has been attempted to calculate thermal distribution and residual stresses using FEM analysis. An axisymmetric model [103] is attempted with the assumption that the powders transform into a sphere due to surface tension. A symmetric boundary condition model [104] with respect to a plane in the vertical direction of scan path has been attempted with consideration of laser light penetration and evaporation. Simulations with a global scale model [105] which averages out the scan pattern into an area heat source has been considered to reduce computational complexity. Such a model will calculate thermal evolution and stresses away from the scan pattern more accurately compared to evolution just near the laser spot. A single scale 3D finite element model [106] has been attempted to simulate thermal distribution and residual stresses along with temperature and porosity dependent thermal properties. 
In the simulation of Metal Laser Sintering, or more generally for spatiotemporal problems, multi scale simulation is necessary to account for multiple macroscopic scales involved in the analysis. A two scale model for subdomain refinement [107] has been attempted with temperature dependent thermal properties and phase transformations. An element birth and death process [108] has been implemented to simulate Metal Laser Sintering along with material nonlinearities in a 3D transient thermal finite element model. The simulation uses a two scale resolution model with tapering in the refinement transition zone. A similar mesh simulation [109] has been attempted for overhanging part build in Metal Laser Sintering with the help of ANSYS. Childs et al. [110] investigated the influence of process parameters on the mass of melted single layers in Metal Laser Sintering and found that melted mass increased with increasing scanning speed. A static two scale model [111] is prepared for predicting melt pool dimensions for a geometry of $6 \times 9 \mathrm{~mm}$ area.

\subsubsection{Spatio-temporal Periodic Problems and Metal Laser Sintering}

A spatio-temporal periodic function is defined as a function which repeats itself over time with rigid translation in space. It can be expressed in the following form.

$$
f(x, t)=f(x+v T, t+T)
$$

Where the $v$ represents the speed and $\mathrm{T}$ represents the time-period of the problem. An assumption for continuously spatio-temporal periodic problems in time is given in equation 45.

$$
\mathrm{T} \rightarrow 0
$$


Spatio-Temporal periodicity can be observed in various problems including metal laser sintering, wave propagations, thermodynamics of welding, various manufacturing processes like sheet metal rolling, and dynamic tire road surface contact. The present work is focused on simulation of metal laser sintering using a novel feed forward dynamic adaptive mesh refinement and de-refinement finite element (FFD-AMRD) algorithm. This algorithm involves a moving fine-mesh box which can capture the spatiotemporal phenomena shown in figure 19.

Metal laser sintering is an additive manufacturing process in which the surface of a powder bed is melted layer by layer to create a 3D part with complex geometry. Further detailed descriptions and literature related to our metal laser sintering thermo-mechanical formulation can be found in a related article [112]. The basic PDE and FEM formulation required to solve thermal behavior during metal laser sintering is given in the next subsection.

\subsubsection{Governing Equations and Finite Element Formulation in FFD-AMRD}

The governing heat transfer equation can be written as

$$
-\left(\frac{\partial q_{x}}{\partial x}+\frac{\partial q_{y}}{\partial y}+\frac{\partial q_{z}}{\partial z}\right)+Q=\rho c \frac{\partial T}{\partial t}
$$

Where $q_{x}, q_{y}, q_{z}$ are components of heat flow through unit area. According to Fourier's law 


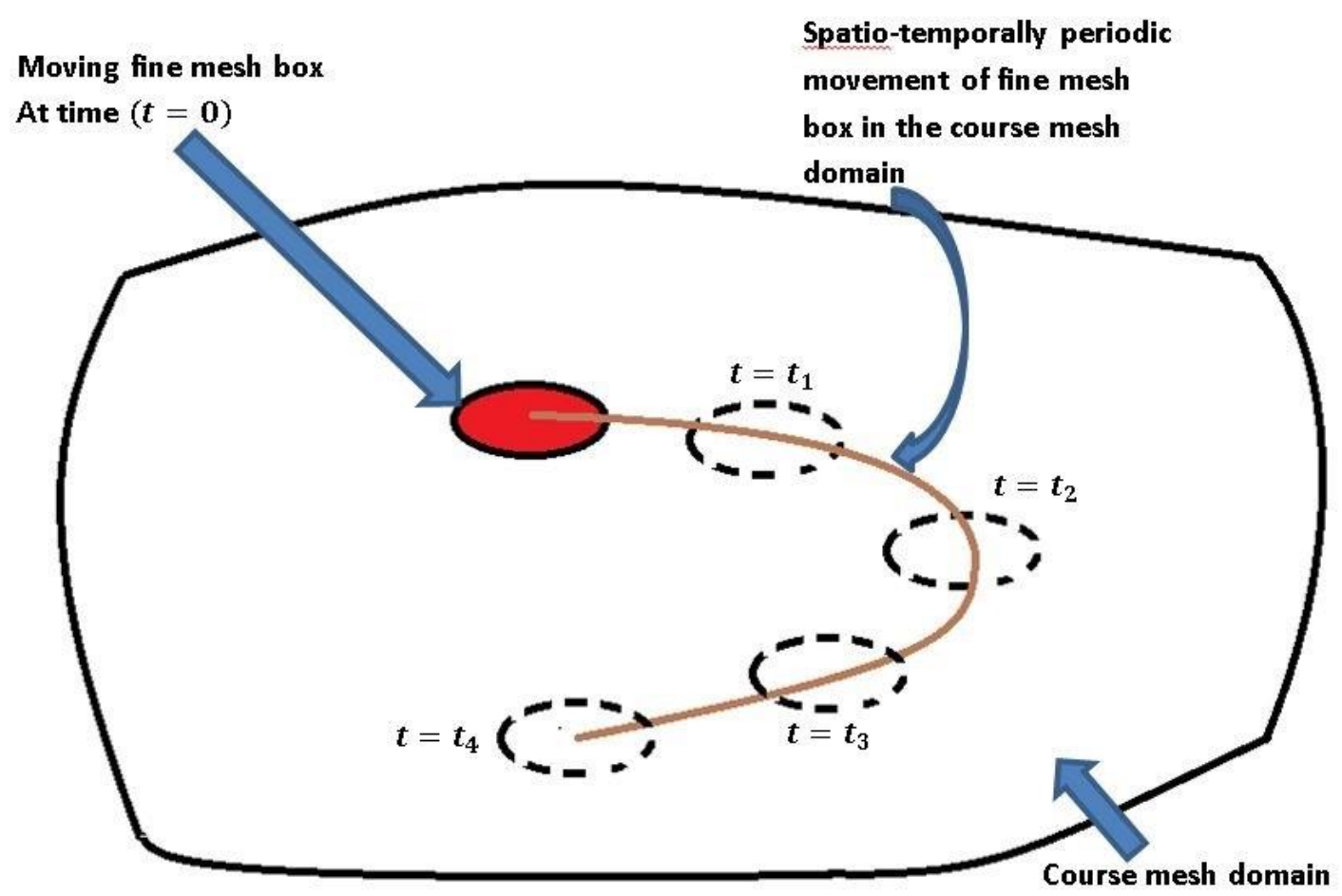

\section{De-refined course}

mesh from previous

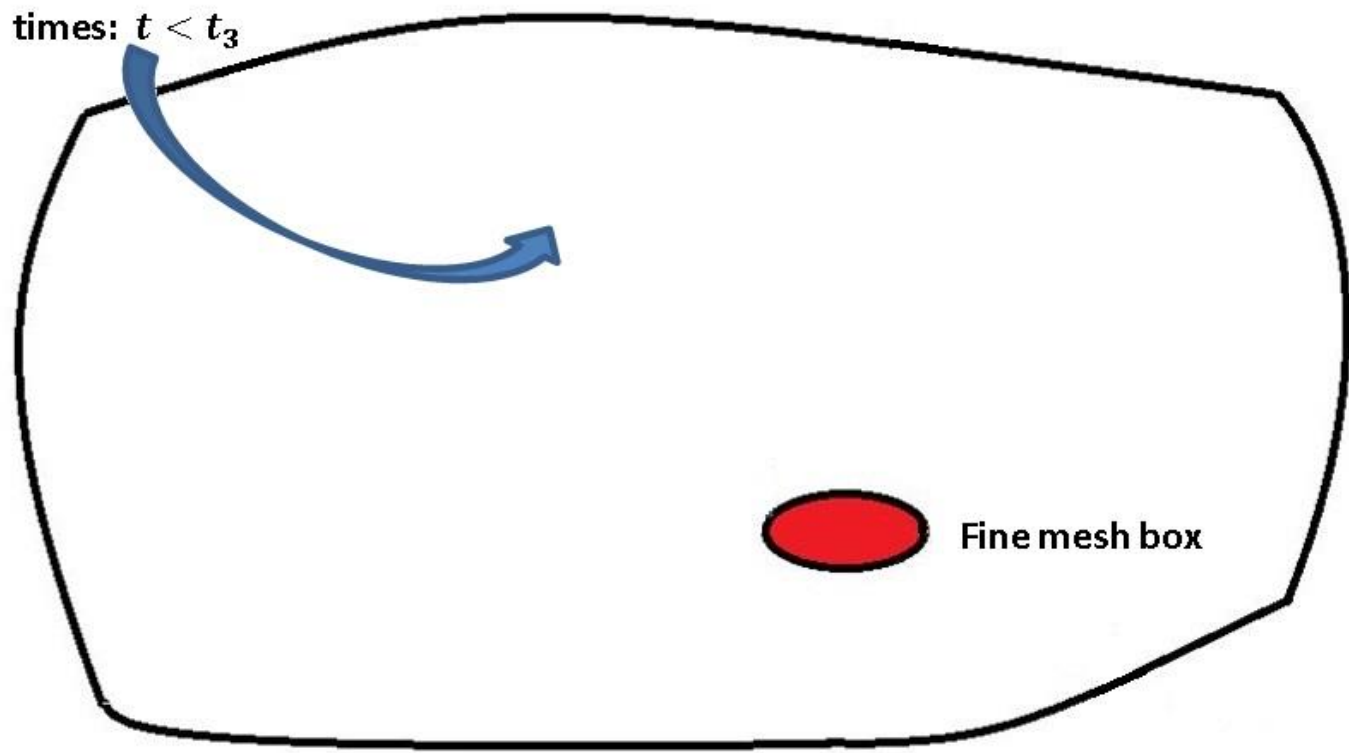

Mesh snapshot at

time $t=t_{3}$

Figure 19 Schematic Diagram showing FFD-AMRD dynamic mesh 


$$
\begin{aligned}
& q_{x}=-k \frac{\partial T}{\partial x} \\
& q_{y}=-k \frac{\partial T}{\partial y} \\
& q_{z}=-k \frac{\partial T}{\partial z}
\end{aligned}
$$

\section{Element Formulations of 3D thermal FEM}

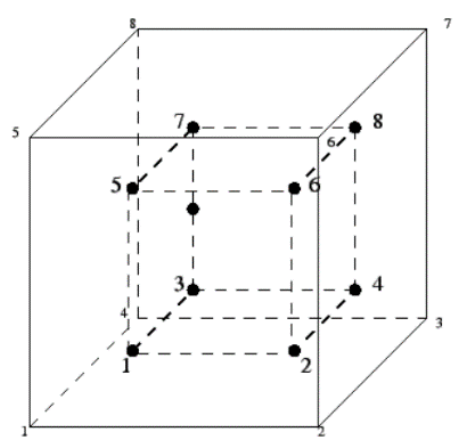

Figure $20 \mathrm{~A}$ brick element with 8 integration points. The $\bar{B}$ integration scheme has been incorporated

Equation 46 has been decomposed into a weak form [113] as equation 48.

$$
\left[C_{\text {thermal }}\right]\{\dot{T}\}+\left[K_{\text {Thermal }}\right]\{T\}=\left\{R_{Q}\right\}
$$

A description of the three matrices in equation 48 is given below.

(i) Thermal stiffness formulation

The thermal stiffness matrix $K_{\text {Thermal }}$ is expressed as follows:

$$
K_{\text {Thermal }}=\int_{V} B_{\text {Thermal }}^{T} \overrightarrow{\vec{k}} B_{\text {Thermal }} d V+\int_{S} h N^{T} N d S
$$


where $B_{\text {Thermal }}$ is known as the flux-temperature matrix. The size of this matrix is $3 \times 8$. For a brick element comprised of 8 integration points as shown in figure 20 , equation 49 is cumulatively repeated for all the integration points leading to $K_{\text {Thermal }}$ of size $8 \times 8 . d V$ denotes the volume of the element. The surface integral is valid when the bulk is exposed to a convection boundary condition. The surface integral is employed at the exposed surface with 2 dimensional shape functions [113] and then transformed to three dimensional space. $h$ in the above mentioned case is the convective heat transfer coefficient which has been assumed to be 12.5 $\mathrm{W} /\left(\mathrm{m}^{2} \mathrm{~K}\right)$ for Argon [114]. Argon fills the chamber atmosphere for reactive materials processed using metal laser sintering.

(ii) Thermal specific heat matrix formulation

The thermal specific heat matrix $C_{\text {thermal }}$ is expressed as follows:

$$
C_{\text {thermal }}=\int_{V} \rho N^{T} c N d V
$$

where $[N]=\left[\begin{array}{lllll}N_{1} & N_{2} & N_{3} & \ldots . . N_{8}\end{array}\right]$ are the 3 dimensional nodal shape functions of size $1 \times 8 . C_{\text {thermal }}$ is computed once and it has a size of $8 \times 8$.

(iii) Thermal flux vector formulation

The thermal flux vector $R_{Q}$ of size $8 \times 1$ is evaluated as follows:

$$
\left\{R_{Q}\right\}=\int_{S}(\vec{q}(\vec{r}, t) \cdot \hat{n}) N^{T} d S+\int_{S} h T_{e} N^{T} d S
$$

where $\vec{q}$ is the input heat flux. $d S$ denotes the surface area of the element. The second surface integral in equation (51) is valid only when the convection boundary 
conditions operate and hence should be employed on those boundaries only. In this scenario, $T_{e}$ denotes the temperature of the ambient environment.

Calculation of these matrices and solving equation 48 requires significant computational effort if uniform fine mesh density is employed. In the next section a proposed efficient numerical algorithm is presented with mathematical error estimation rationality for its development.

\subsection{FFD-AMRD framework for applications in spatio-temporally periodic localized boundary conditions}

In this section the FFD-AMRD algorithm is described and applied to metal laser sintering. First a mathematical rationality for using refined moving fine mesh is established. The thermal behavior in the case of metal laser sintering can be decomposed into local and global problems in terms of thermal curvature. It can be approximately estimated using a cylindrical coordinate system in $2 \mathrm{D}$. Here thermal flow is assumed to be 2-dimesional and the Gaussian heat flux from the laser is assumed to be an external heat input as $h(r)$.

$$
k \frac{d^{2} T}{d r^{2}}=\frac{d T}{d t}-k \frac{1}{r} \frac{d T}{d r}-h(r)
$$

The laser is centered at $r=0$ with Gaussian flux. The $\frac{d T}{d r}$ term in the above equation has a magnitude on the order of $\left(\frac{1}{r^{2}}\right)$ provided the integral of the radial flux is maintained constant. The assumption that laser flux is equal to direct heat input $h(r)$ with Gaussian distribution can be described as:

$$
h(r)=c e^{-\frac{r^{2}}{2 \sigma^{2}}}
$$


Substituting equation 53 into equation 52 and using an order of magnitude analysis along with equations 7 and 10 results in equation 54 .

$$
\vartheta\left(k \frac{d^{2} T}{d r^{2}}\right)=-\vartheta\left(\frac{1}{r^{2}}\right)-\vartheta\left(c e^{-\frac{r^{2}}{2 \sigma^{2}}}\right) \approx \vartheta\left(h^{2}\right)
$$

This asymptotic behavior can be further exploited to increase efficiency of otherwise computationally expensive spatiotemporally periodic problems. The algorithm for achieving the increased efficiency is described in the next two sub-sections. A nonlinear asymptotic expansion decomposition of this problem has been attempted [112].

\subsubsection{Fast Sorting Methodologies to Increase Data Transfer Between Meshes}

The algorithm for interpolation of DOF information from a node in a previous spatial mesh configuration to the next spatial mesh configuration depends on the implemented method. In the present work, an inverse isoparametric interpolation strategy has been used. This strategy searches a target element in the next spatial mesh configuration in close proximity to the nodes present in the previous spatial mesh configuration. This search is expensive to perform though most of the nodes in the coarse mesh have a one-to-one correspondence between the present spatial mesh configuration and the next one. Henceforth, the number of calls for searching a target element in the next mesh configuration is largely reduced. Moreover, the search is not exhaustive in nature. The element in the next mesh configuration has defined bounds and the nodes falling between those bounds are used for interpolation as shown in the schematic in Figure 21. 


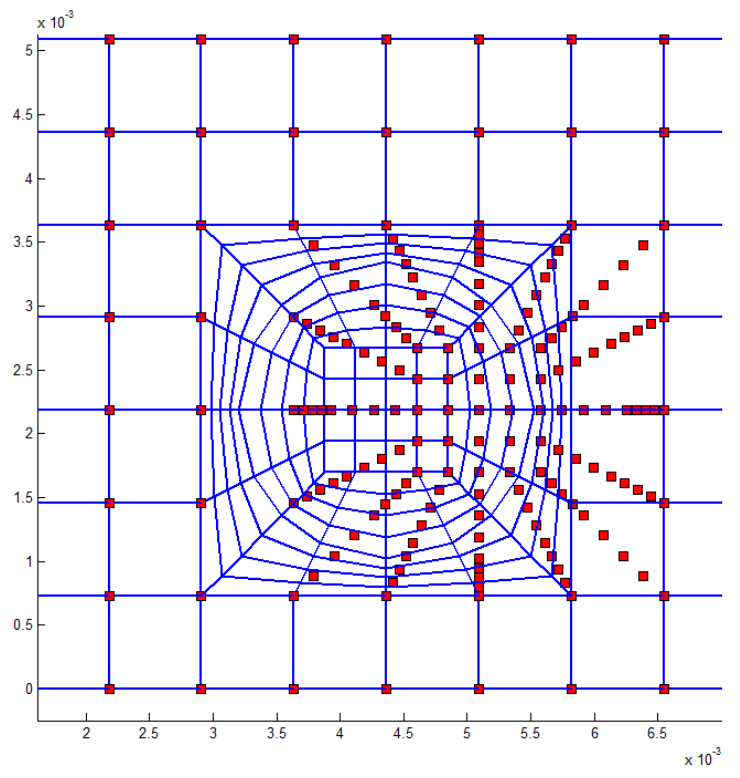

Figure 21 Schematic showing old (blue elements) and next (red nodes) mesh configurations.

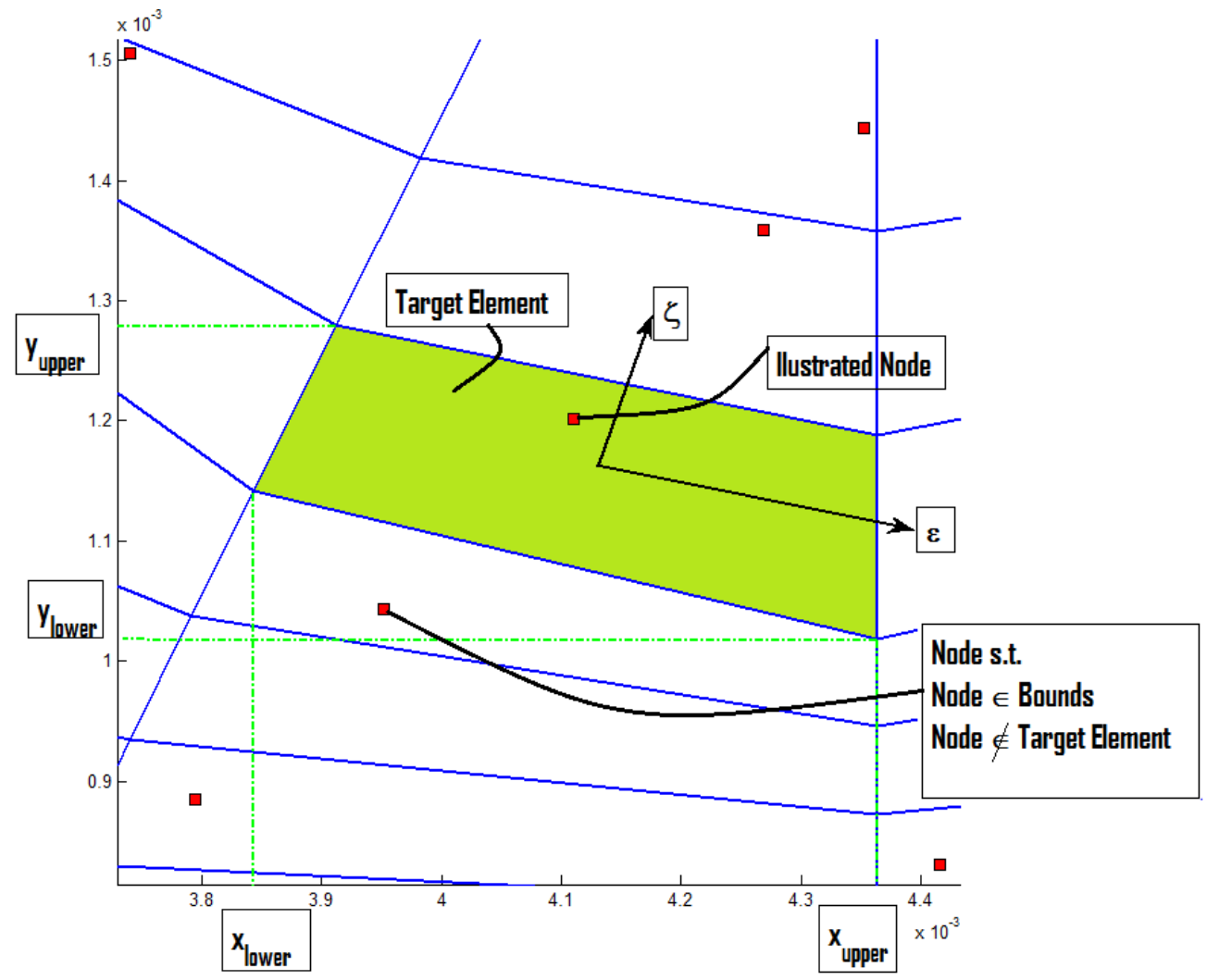

Figure 22 Target Element (old mesh configuration) for illustrated nodes (next mesh configuration). $\mathrm{x}$ and $\mathrm{y}$ bounds are shown with local isoparametric target element axes $\zeta$ and $\varepsilon$. 
The fast sorting strategy is described using set theory based mathematical equations from equations 28 to 37 .

$$
\begin{aligned}
& \operatorname{Mesh}_{o}=f\left(N_{o}, E_{o}\right) \\
& \operatorname{Mesh}_{N}=f\left(N_{N}, E_{N}\right)
\end{aligned}
$$

where subscript $\mathrm{O}$ denotes the previous (old) mesh configuration and subscript $\mathrm{N}$ denotes the next (new) mesh configuration. Figure 21 shows nodes in two different consecutive configurations. Mesh denotes the connectivity of node $(\mathrm{N})$ and element (E) respectively. Each component of the mesh array (Mesh) consists of 1 element number and 8 node numbers in a strictly positive triple product sequence.

$$
\operatorname{cord}(N)=\left[X_{N}, Y_{N}, Z_{N}\right]
$$

where the $\operatorname{cord}(N)$ function denotes the 3 dimensional location of a particular node $(\mathrm{N})$ consisting of a position vector triplet $\left[X_{N}, Y_{N}, Z_{N}\right]^{T}$.

$$
\begin{gathered}
\operatorname{cord}\left(N_{O}\right) \cap \operatorname{cord}\left(N_{N}\right)=G \\
N_{C}=\operatorname{cord}^{-1}(\mathrm{G})
\end{gathered}
$$

where node $\left(N_{C}\right)$ denotes the common nodes between the previous and next mesh configurations.

$$
f\left(N_{C}, E_{o}\right)=f\left(N_{C}, E_{N}\right)
$$

which solves for the elements $E_{C}$, common to both previous and next mesh configurations 


$$
E_{o}-E_{C}=E_{N C O}
$$

where element $\left(E_{N C O}\right)$ denotes the uncommon nodes present in the previous mesh configuration.

$$
N_{N}-N_{C}=N_{N C N}
$$

where node $\left(N_{N C N}\right)$ denotes the uncommon nodes present in the next mesh configuration.

$$
\begin{aligned}
& \quad \operatorname{Bound}\left(E_{N C O}\right)=\left[X_{\text {ini,NCO }}, X_{\text {ini,NCO }}, Y_{\text {ini,NCO }}, Y_{\text {ini,NCO }}, Z_{\text {ini,NCO }}, Z_{\text {ini,NCO }}\right] \\
& \forall \operatorname{cord}\left(N_{N C N}\right) \in \operatorname{Bound}\left(E_{N C O}\right): \text { as shown in (figure 22) }
\end{aligned}
$$

There exists a pair s.t.

$$
\operatorname{Pair}(N C N, N C O)=\left[N_{N C N}, T\left(N_{N C N}\right)\right]
$$

where

$$
T\left(N_{N C N}\right) \subset E_{N C O}
$$

\subsubsection{Efficient Stiffness Matrix Assembly}

The stiffness matrix of the dynamic moving mesh for a particular $i^{\text {th }}$ mesh configuration is generated from the intact course mesh and fine mesh stiffness matrices. This will reduce the actual computational time required to assemble stiffness matrices.

\subsubsection{FFD-AMRD thermal FEM algorithm}

In the FFD-AMRD algorithm, the refinement zone is expected to refine and de-refine itself as the external spatiotemporal stimulus or response moves. Table 2 explains the 
intelligent algorithm to efficiently perform such refinement and de-refinement in negligible time overhead for FEM mesh regeneration and renumbering along with recalculation of FEM matrices.

Step 1: Mesh Generation:

Table 2 FFD-AMRD algorithm

1. Fixed coarse mesh generation $\boldsymbol{a} \times \boldsymbol{b} \times \boldsymbol{c}$ size prism

- $\operatorname{div}_{x}, \operatorname{div}_{y}$ and $\operatorname{div}_{z} ; 2 \mathrm{D}$ element connectivity (Matrix: \# of Coarse Elements (Coarse_el_length) $\times$ 8)

- $\operatorname{node}_{x}$, node $_{y}$ and node $_{z}$

- 2D mesh for bottom surface -- 2D connectivity (elem) and coordinate (coord) arrays

- Extrude to make 3D mesh -- 3D elem and coord arrays (figure 23)

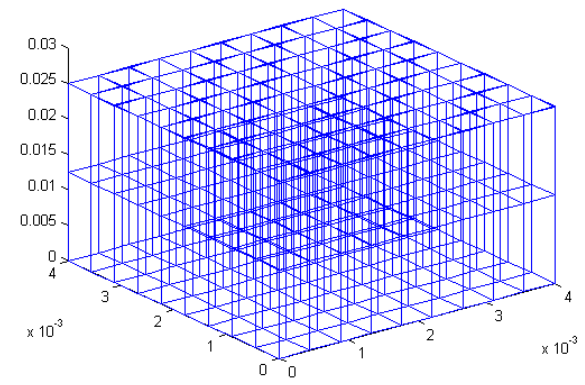

Figure 23 3D extruded fixed coarse mesh generation

2. Dynamic fine mesh block $\boldsymbol{S} \boldsymbol{d} \times \boldsymbol{S d}$ size prism

- Decide mesh divisions

- Generate bottom surface mesh : 

i. nodes for the central square box
ii. nodes for radial boxes
iii. element connectivity for each block

- Extrude in $z$ direction

- elem and coord (figure 24)

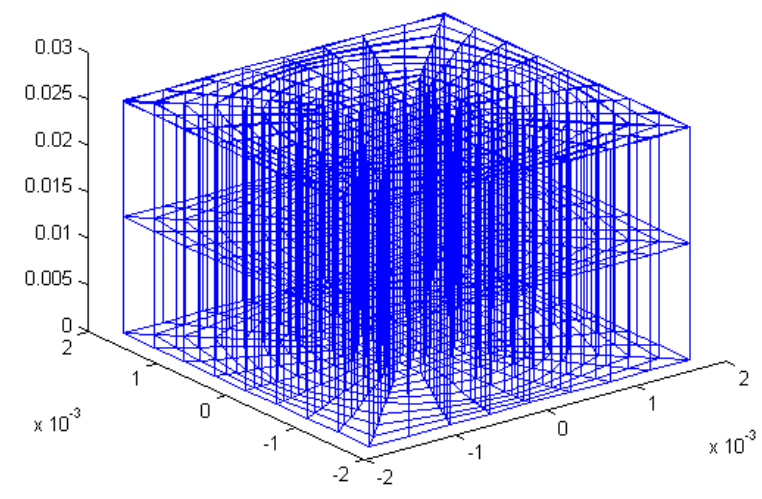

Figure 24 3D extruded independent fine mesh generation

3. Combined Adaptive Mesh for laser beam at location $\vec{r}=\vec{r}_{q}+\vec{v} t$

- Cut elements cut_list(Vector: Evac $\times 1$ ) from coarse mesh

- Paste finemesh

- GlobalConnectivity (Matrix: Numelem $\times$ 8) array (figure 25)

- GlobalCoord (Matrix: tlnodes $\times 3$ ) array (figure 25) 


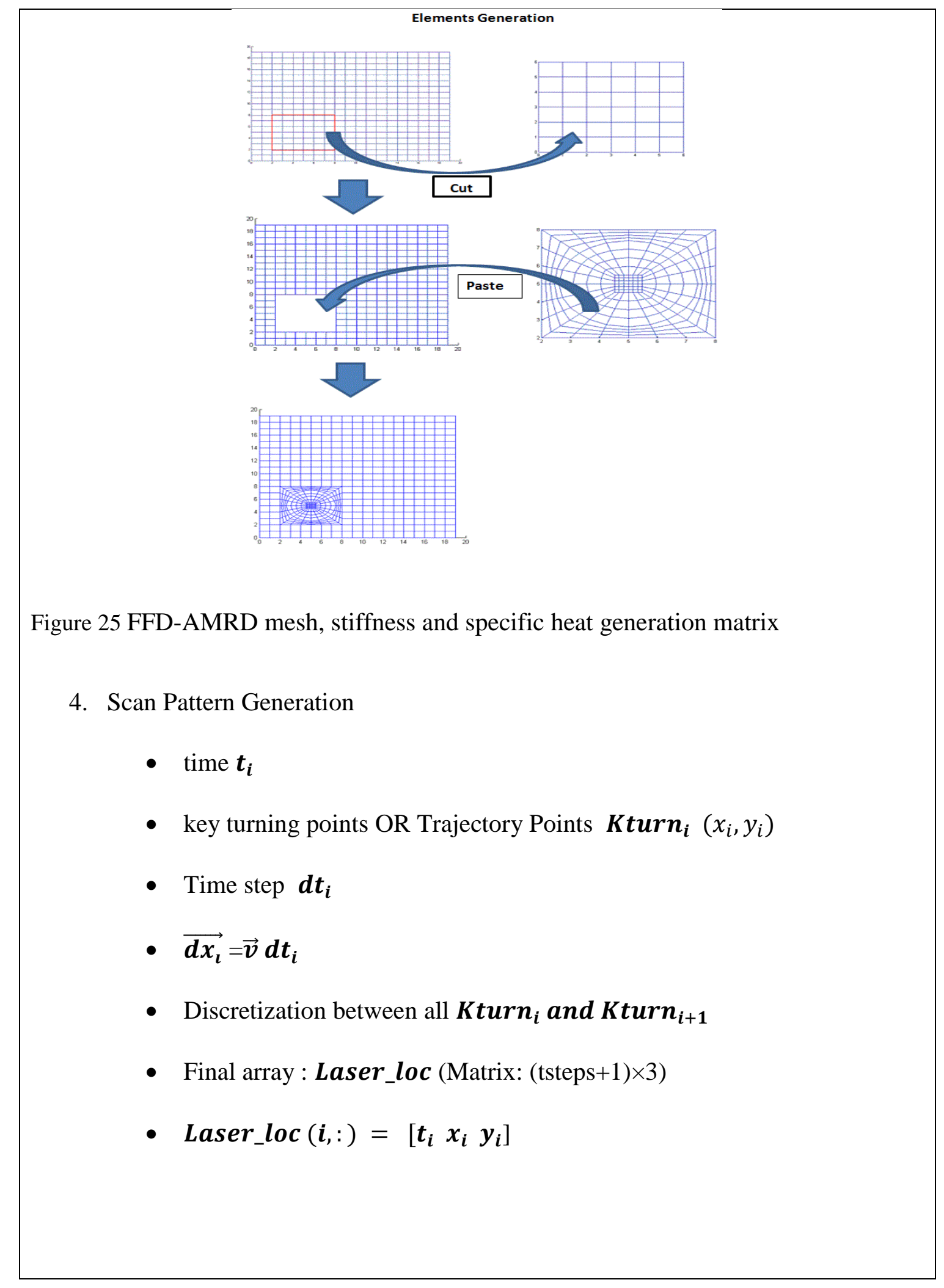


FOR layer $=1$ to \#layers (Number of layers)

Modify: elem and coord for added layer

Decide: dimensions of all arrays like $\mathbf{K}$ and $\mathbf{C}$

Generate $K_{\text {coarse }}, K_{\text {fine }}, C_{\text {coarse }}, C_{\text {fine }}$

Generate $K_{v a c}$ and $C_{v a c}$

FOR offset $=1$ to \#finemesh movements

from last mesh to new mesh: isoparametric interpolation

i. DOF Transfer

ii. State variable

FOR timestep: $t_{i}$ to $t_{i+n}$

Generate Nonlinear K and C matrices

$$
K_{\text {pred }}=f\left(K_{\text {coarse }}\right)+A\left[K_{\text {fine-norm }}\right] B+
$$

$g(T$, state variables $)$

Simplify in the form of $K_{\text {pred }}=K+\Delta K$ 


$$
\begin{gathered}
T_{(i+1) \text { pred }}=\text { cholesky }\left(K_{\text {pred }}\right) T_{i} \\
K_{\text {corrected }}=f\left(K_{\text {coarse }}\right)+A\left[K_{\text {fine-norm-corrected }}\right] B+ \\
g(T, \text { state variables }) \\
T_{(i+1) \text { correctd }}=f^{\prime}\left(K_{\text {corrected }}\right)
\end{gathered}
$$

Interpolation to simple regular base grid: For input to DDCP

FEM

\author{
DOF Transfer
}

State Variable Transfer

\title{
END timesteps
}

END offsets

FOR 1 to \# cooling time steps

Generate Nonlinear $\mathrm{K}$ and $\mathrm{C}$ matrices for the fine mesh region

$$
\begin{gathered}
K_{\text {pred }}=f\left(K_{\text {coarse }}\right)+A\left[K_{\text {fine }- \text { norm }}\right] B+g(T, \text { state variables }) \\
\text { Simplify in the form of } K_{\text {pred }}=K+\Delta K
\end{gathered}
$$




$$
\begin{gathered}
T_{(i+1) \text { pred }}=\text { cholesky }\left(K_{\text {pred }}\right) T_{i} \\
K_{\text {corrected }}=f\left(K_{\text {coarse }}\right)+A\left[K_{\text {fine-norm-correctd }}\right] B+ \\
g(T, \text { state variables }) \\
T_{(i+1) \text { correctd }}=f^{\prime}\left(K_{\text {corrected }}\right)
\end{gathered}
$$

END time steps

END Layers

\subsubsection{Comparison of FFD-AMRD algorithm with traditional FEM}

Figure 25 shows that fine mesh box divisions (Fd) of the course mesh are transitioned in to the same number of divisions. The zoom in ratio in both $\mathrm{x}$ and $\mathrm{y}$ directions $(\mathrm{Zr})$ will determine the computational complexity when compared with uniform mesh FEM analysis. The computational complexity [115] of Cholesky Decomposition for solving (n) number of simulation equations is $\left(n^{3} / 2\right)$. This implies that the time complexity comparison of a uniform mesh compared with FFD-AMRD will be exactly $\left(Z r^{6}\right)$. This is based on the fact that the ratio of number of nodes will be equal to $\left(Z r^{2}\right)$. In the present work since $\mathrm{Zr}$ equal to 3 is used, the computational complexity ratio between traditional FEM and FFD-AMRD will be 729.

\subsection{Problem geometry description and boundary conditions}

Two case studies were attempted. The first is a linear one dimensional FEM analysis approximation of spatiotemporally periodic heat input and is referred to as Case Study 1. 
This analysis will serve as a simplified case for developing a phenomenological understanding of various nonlinearities and for testing algorithms. The second case study involves a three dimensional FEM simulation using the FFD-AMRD algorithm to simulate metal laser sintering and is referred to as Case Study 2. In both case studies heat input (machine process) parameters are assumed as follows.

scan speed: $1200 \mathrm{~mm} / \mathrm{s}$,

beam diameter: $100 \mu m$

hatch spacing: $100 \mu \mathrm{m}$

Laser power: 180 watts

\subsubsection{Case Study 1}

The problem geometry considered here is a one dimensional bar. The assumption of a one dimensional bar is valid since the geometry considered is a straight bar with small cross-sectional dimensions compared to its length (1). Figure 26 shows the problem geometry.

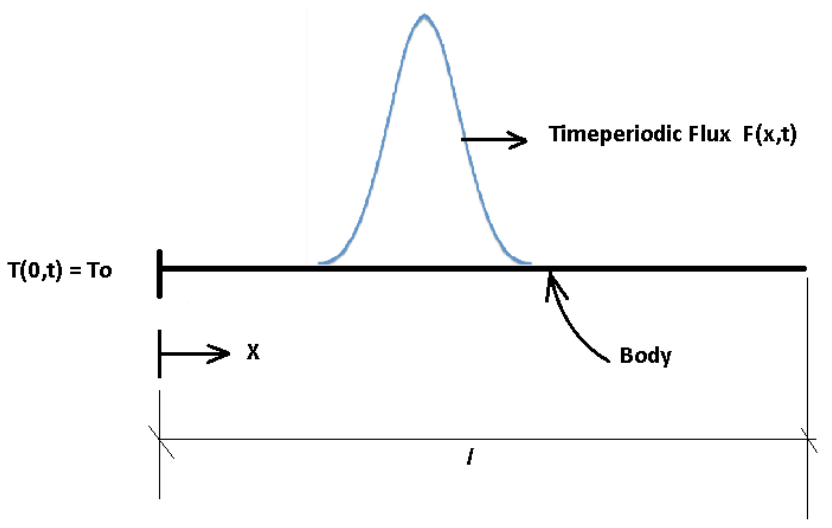

Figure 26 Dynamic boundary condition of the one-dimensional problem 
The basic equation of heat transfer is

$$
\frac{\partial T(x, t)}{\partial t}=\frac{k}{c \rho} \frac{\partial^{2} T(x, t)}{\partial x^{2}}+Q(x, t)
$$

where

$T(x, t)=$ Temperature at point $\mathrm{x}$ and time $\mathrm{t}$.

$k=$ One-dimensional material conductivity

$c=$ One-dimensional specific heat of the material

$\rho=$ Density of the bar for a unit cross-section

$Q(x, t)=$ Inner heat generation at point $\mathrm{x}$ and time $\mathrm{t}$.

Also, we define another variable to incorporate the flux boundary conditions as follows:

$$
q(x, t)=-k \frac{\partial T(x, t)}{\partial x}
$$

where $q(x, t)=$ Flux at point $\mathrm{x}$ and time $\mathrm{t}$.

Further, the spatio-temporal periodicity of the flux boundary is considered as:

$$
q(x+v(d t), t+d t)=q(x, t)
$$

The Dirichlet boundary condition considered here is constant temperature at the left end of the bar as shown in figure 26. The Dirichlet boundary condition is required so that the thermal stiffness matrices, namely the conduction and specific heat matrices, become nonsingular. 


$$
T(0, t)=T_{0}=353 \text { Kelvins }
$$

The time-periodic Neumann boundary condition considered here is the laser flux distribution in one dimension:

$$
q(x, t)=\left(\frac{2 P}{\pi r_{\text {laser }}^{2}}\right) \exp \left(\frac{-2(x-v t)^{2}}{r_{\text {laser }}^{2}}\right)
$$

where $P=$ Laser beam power $=180 \mathrm{~W}$

$r_{\text {laser }}=$ Laser beam spot size incident perpendicular to the length $(1=100 \mathrm{~mm})=100 \mu \mathrm{m}$ $v t=$ Distance travelled by the laser beam from the left end of the bar with a speed $(v=$ $1200 \mathrm{~mm} / \mathrm{s}$ ) at time instant $t$

The initial condition for the bar considered here is constant temperature $T_{0}$.

$$
T(x, 0)=T_{0}=353 \text { Kelvins }
$$

\subsubsection{FEM discretization and FEM formulation}

The bar is discretized into $p+1=2000$ nodes as shown in the figure 27 . All elements are of the same length so element matrices such as conduction and specific heat matrices involved in FEM will be the same for all elements. 


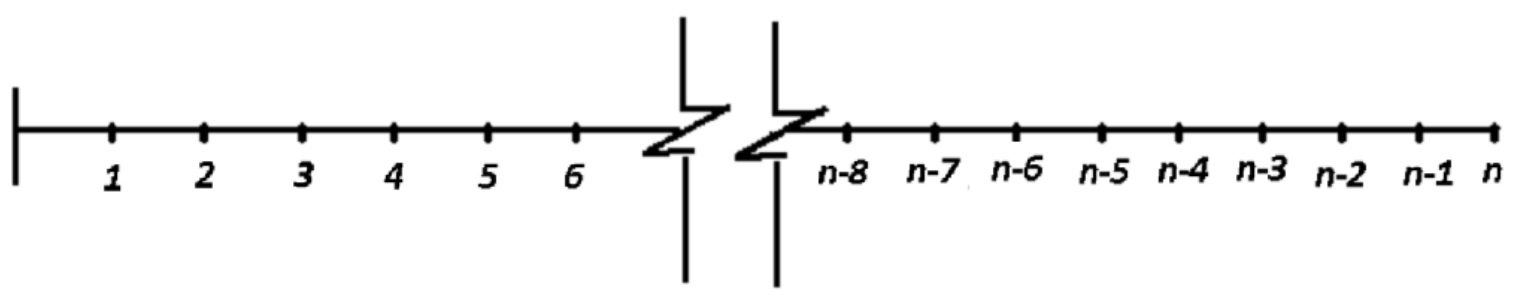

Figure 27 1-Dimensional line elements.

A typical $\mathrm{C}$ matrix of an element is as follows:

$$
C=\left[\begin{array}{cc}
a & -\operatorname{coeff} c * a \\
-\operatorname{coeffc} * a & a
\end{array}\right]
$$

A typical $\mathrm{K}$ matrix of an element is as follows:

$$
K=\left[\begin{array}{cc}
b & -\operatorname{coeff} k * b \\
-\operatorname{coeffk} * b & b
\end{array}\right]
$$

where $a$ is the specific heat capacity of a node when increasing its temperature at a rate of $1{ }^{\circ} \mathrm{C} / \mathrm{s}$ and $\operatorname{coeff} \mathrm{c}$ is the interaction specific heat capacity between the connecting nodes. Similarly, $b$ is the amount of flux flowing through a node when increasing its temperature gradient at a rate of $1{ }^{\circ} \mathrm{C} / \mathrm{m}$ and coeffk is the interaction flux between the connecting nodes. coeffc and coeffk are generally considered 0.5 for the one dimensional case since the probability of heat flow is equal on both sides of the node. Since a one-dimensional approximation of the three dimensional heat transfer mechanisms of the metal laser sintering process are considered in the one-dimensional bar problem, the above mentioned coefficients will show drastic reduction in their values. In the current problem, the coefficients have been reduced by homogenizing a probability distribution such that it allows more heat transfer in the bulk as compared to the surface. 


\subsubsection{Case Study 2}

Detailed descriptions of Case Study 2 have been provided in a prior publication [112]. A thermally linear, homogenous and isotropic material behavior has been assumed for the Ti6A14V material. The powder, molten metal inside melt pool and solidified regions have been assumed to be distinct homogenous phases. The geometry of the problem is a solid base plate of thickness $25.4 \mathrm{~mm}$ and a powder layer of thickness $30 \mu \mathrm{m}$. A subdomain of full powder bed of size $2 \mathrm{~mm} \times 2 \mathrm{~mm}$ has been considered for simulation.

\subsection{Results and Discussion}

\subsubsection{Case Study 1}

Two subcases were attempted with constant and temperature dependent thermal parameters. They have been described as follows:

Subcase 1: Thermal material parameters assumed to be constant

Subcase 2: Nonlinear temperature dependent thermal material parameters [112] are considered.

Temperature Distribution: Figure 28 shows the temperature distribution of the one dimensional bar considered here for both the subcases described above and at three equidistant time instances of $0.0025 \mathrm{~s}, 0.005 \mathrm{~s}$ and $0.0075 \mathrm{~s}$. All three time instances are plotted when the melt-pool distribution has achieved its steady state over time. Temperatures in all cases are normalized by dividing by the maximum temperature in the linear case over time. The rationale for doing this normalization is to make results more generalized and meaningful to compare the response between the linear and nonlinear simulations. 


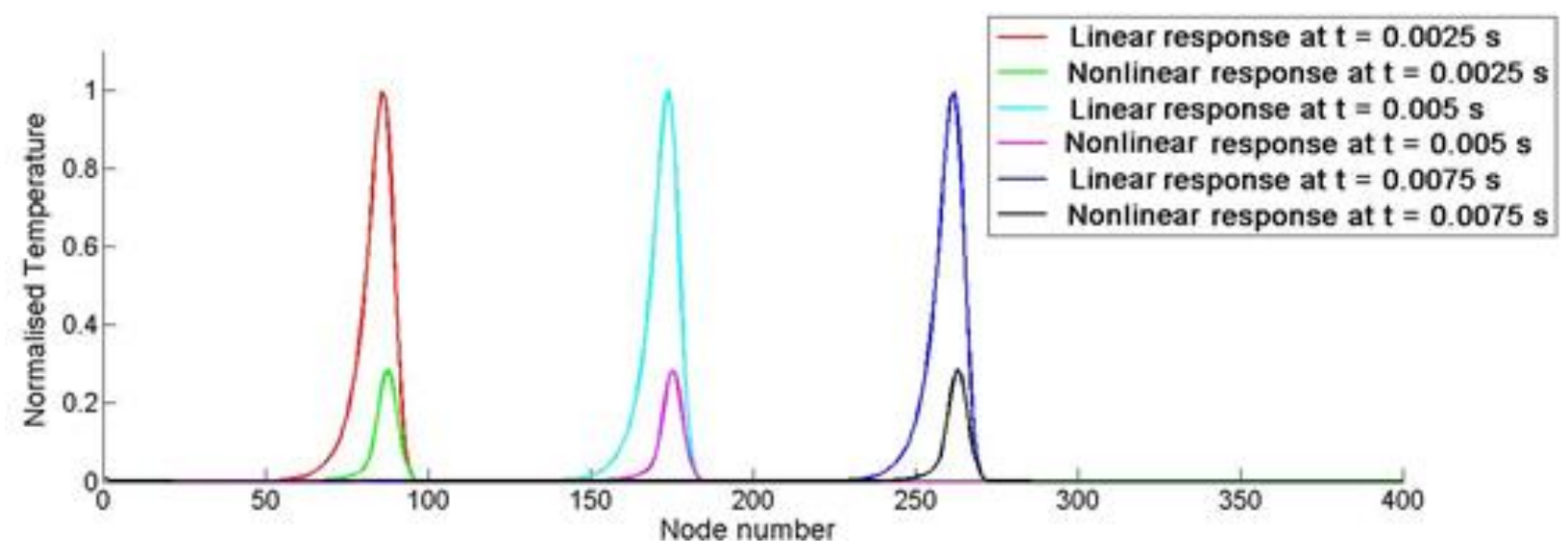

Figure 28 Temperature distribution in 1D space

It can be observed from figure 28 that the temperatures when using nonlinear parameters are significantly lower than when using linear parameters. The reason for this behavior can be attributed to increased conductivity and volumetric heat capacity near the laser beam spot as shown in figures 29 and 30, which leads to faster heat dissipation near the melt pool area. As a result, the melt pool diameter is comparatively smaller in subcase 2 .

Thermal Conductivity: The thermal conductivity remains constant as assumed over time and space in subcase 1 whereas in subcase 2 , it shows a top-hat distribution due to constant thermal conductivity at temperatures higher than $1923 \mathrm{~K}$ (the melting temperature) as shown in figure 29. 


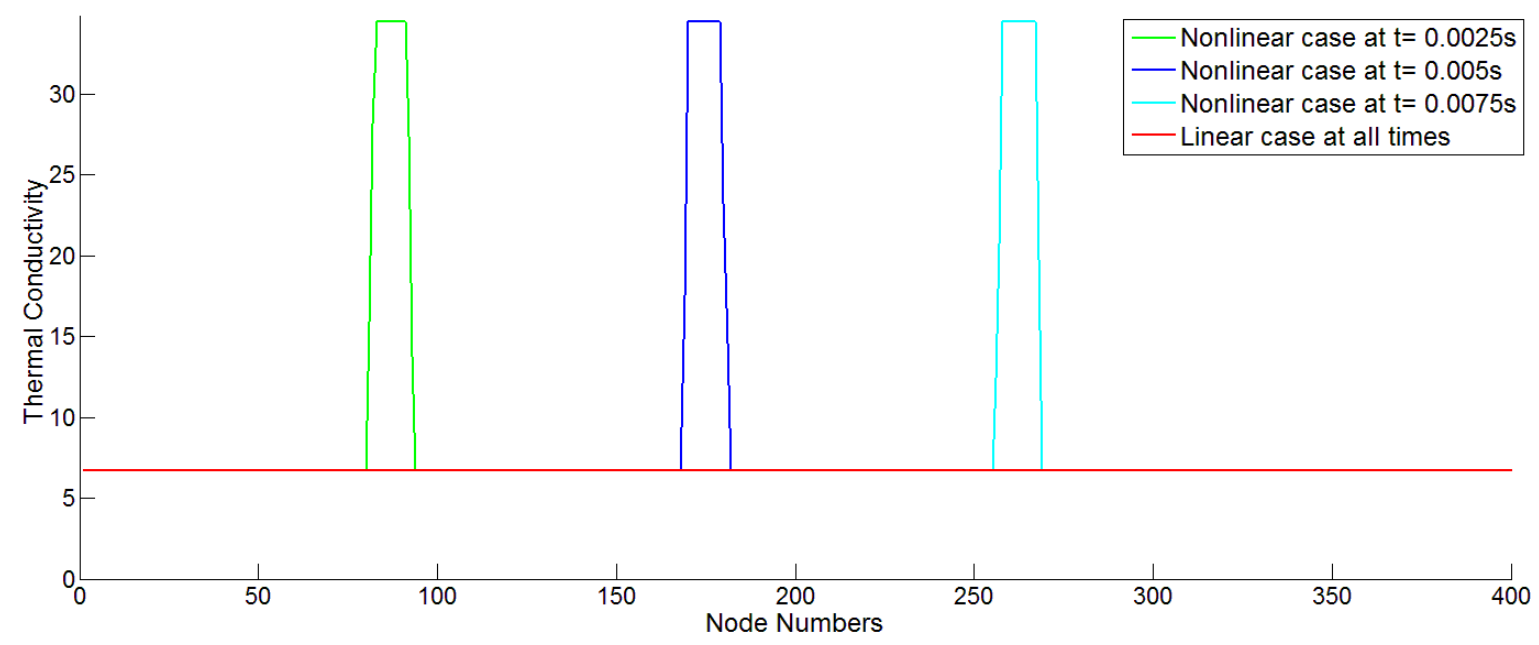

Figure 29 Thermal conductivity distribution for nonlinear and linear cases of the one dimensional problem at three different times plotted against nodes.

Volumetric heat capacity: The non-linear phenomenon in section 3.3 has led to a reverse top hat distribution near the melt-pool as shown in Figure 30.

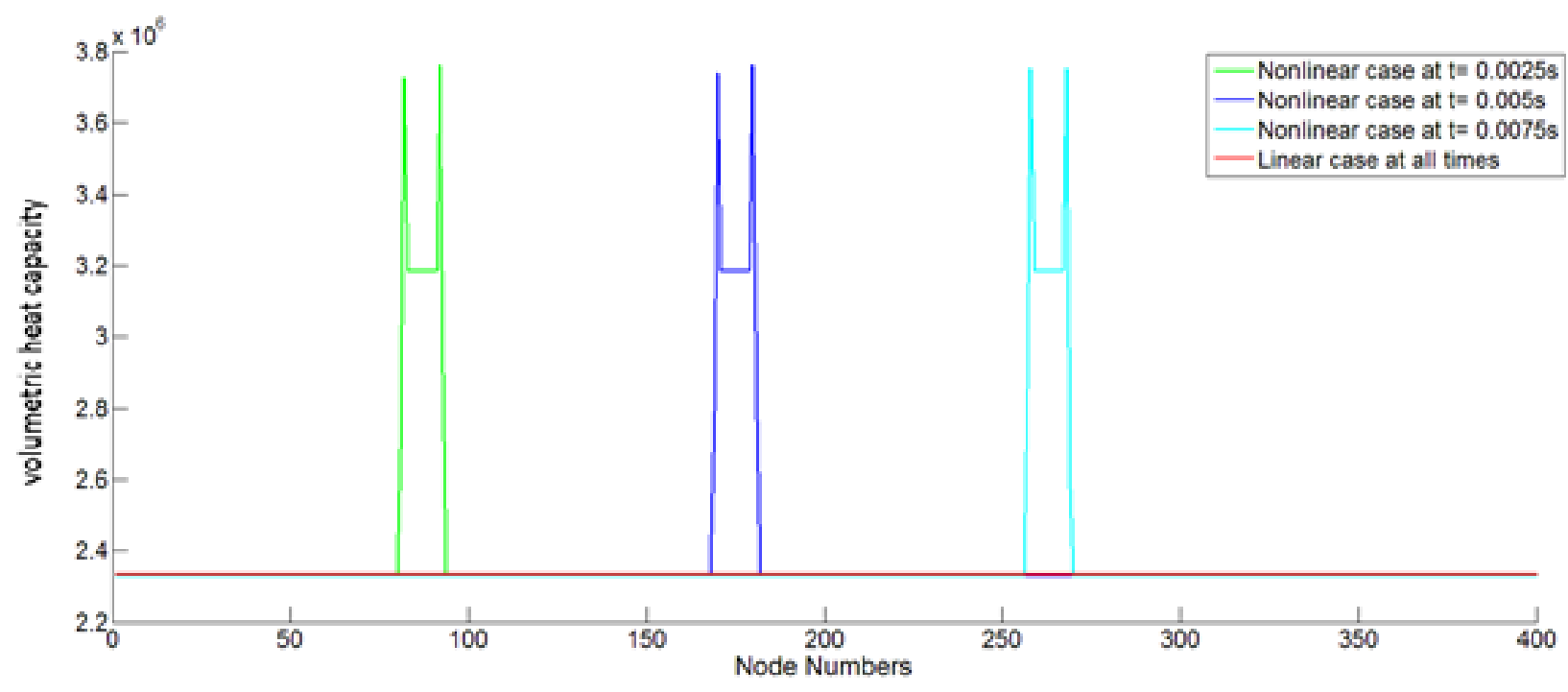

Figure 30 Volumetric heat capacity $(\rho c)$ for nonlinear and linear cases of the one dimensional problem at three different times plotted against nodes 
4.4.2 Three dimensional FEM description of spatio-temporally periodic metal laser sintering problem with Ti6ALAV material

A three-dimensional simulation using the FFD-AMRD algorithm is performed for case study 2. Figure 31 shows the surface temperature contours for the stabilized melt pool. It can be seen that melt pool diameter is $125 \mu \mathrm{m}$.

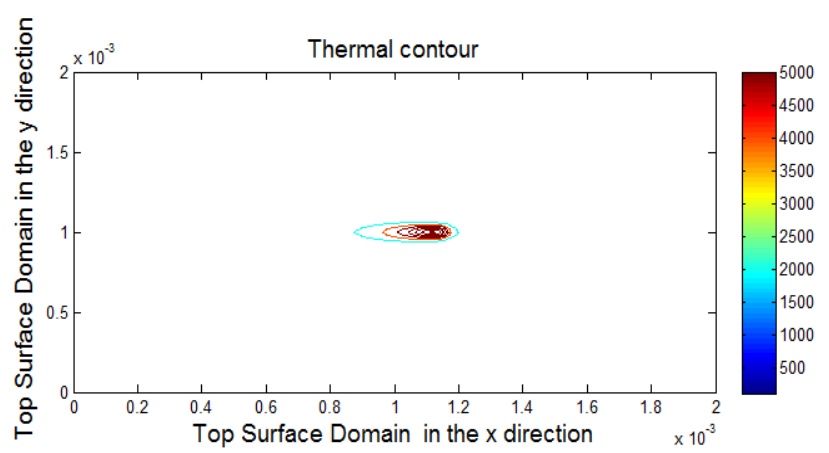

(a)

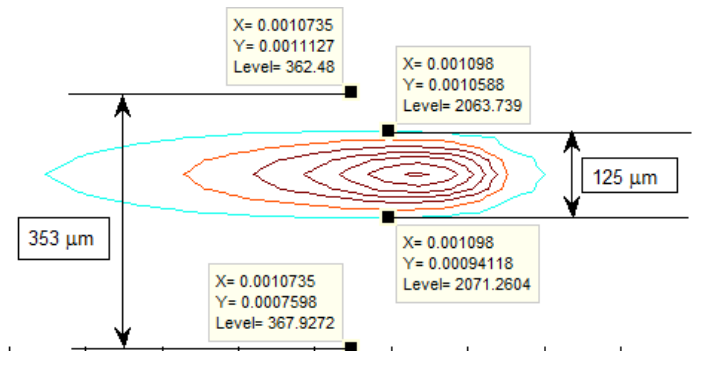

(b)

Figure 31 Comparison of thermal contours. The melt pool diameter in (b) is $125 \mu \mathrm{m}$.

\subsubsection{Microstructural validation}

Microstructural samples were created using a commercial EOS M270 metal laser sintering machine. These samples were fabricated using the same set of parameters used in the simulation and then cut to illuminate the transverse section. A set of cylindrical samples fabricated horizontally in the xy-plane of the machine (normal direction $+x$ ) were made. The prior beta grain size should be the lesser of the melt pool width or the hatch spacing, and the fact that it is equal to the hatch spacing indicates that the melt pool width is wider than the hatch spacing as predicted to be $125 \mu \mathrm{m}$. 


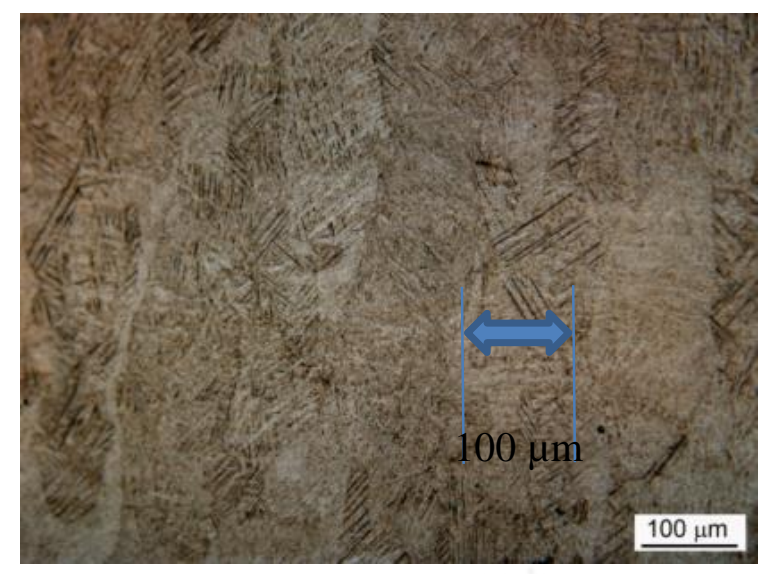

Figure 32 Optical microscopy image of microstructure showing grain boundaries along the melt pool boundary.

\subsection{Conclusion}

The developed numerical technique has shown significant potential for efficient and high resolution simulation of spatio-temporal problems with rapid time and spatial gradients. The magnitude of the temporal temperature gradients are on the order of $10^{5} \mathrm{C} / \mathrm{s}$. Since the temperature profile is approximately Gaussian near the melt pool and is moving at the speed of $1200 \mathrm{~mm} / \mathrm{s}$, traditional FEM analysis is found be 364.5 times slower compared to the FFD-AMRD algorithm derived in section 4.2.3. It is seen in the line problem approximation that linear approximation gives higher temperatures and melt pool diameters. The prior beta grain size should be the lesser of the melt pool width or the hatch spacing, and the fact that it is equal to the hatch spacing indicates that the melt pool width is wider than the hatch spacing as predicted to be $125 \mu \mathrm{m}$ in analysis. Further development of nonlinear FFD-AMRD will help give correct temperature distributions during processing. 


\title{
CHAPTER 5
}

\section{A NEW FINITE ELEMENT SOLVER USING}

\author{
NUMERICAL EIGEN MODES FOR FAST
}

\section{SIMULATION OF ADDITIVE MANUFACTURING}

\section{PROCESSES}

\subsection{Introduction}

Additive manufacturing has been on the forefront of innovations in engineering and has immense potential to revolutionize manufacturing. The major factors that are critical to any manufacturing technology are geometrical accuracy, mechanical properties of the produced part and process speed. These factors are important for additive manufacturing processes as well and improvement in any of these areas can lead to better optimized and reduced cost products. Process optimization based on these factors requires a good understanding of the physics and process behavior involved. The process behavior includes variation of mechanical and thermal variables across the part domain. Process behavior depends on the part geometry and machine parameters. Simulations can help achieve real time optimization of process parameters and closed loop control. However, real time optimization requires fast simulations to be able to work at the speed of the machine. In the present work a new fast and memory efficient numerical solver for the Finite Element 
Method (FEM) is presented for processes involving prismatic process domains including, for instance, the full powder bed in the case of Selective Laser Melting.

\subsection{Selective Laser Melting}

SLM is an important additive manufacturing process in which a laser beam selectively melts a powder bed layer-by-layer to build a complex three dimensional geometry (see Figure 33). SLM has found many applications in complex aerospace parts and biomedical implants. The accuracy and microstructure of the parts manufactured in the process can be improved with better control of machine input parameters. Input parameters for SLM include geometry, thermal boundary conditions, beam power, scan speed and hatch pattern. Optimization of beam power, scan speed and hatch pattern, in particular, affect the thermal behavior of the powder bed and the melt pool shape. The Melt pool shape has a significant impact on the final geometry of the built part. The thermal behavior of the full powder bed is also important from the perspective of thermal cooling rates during solidification, which determines the microstructures and phases in the final part. A detailed literature review of this area has been performed [116]. A recently developed multi-scale simulation framework known as Feed Forward Adaptive Mesh Refinement and De-refinement (FFDAMRD) $[117,118]$ is used in the present work for various simulations and is also used as a part of the proposed simulation framework. 


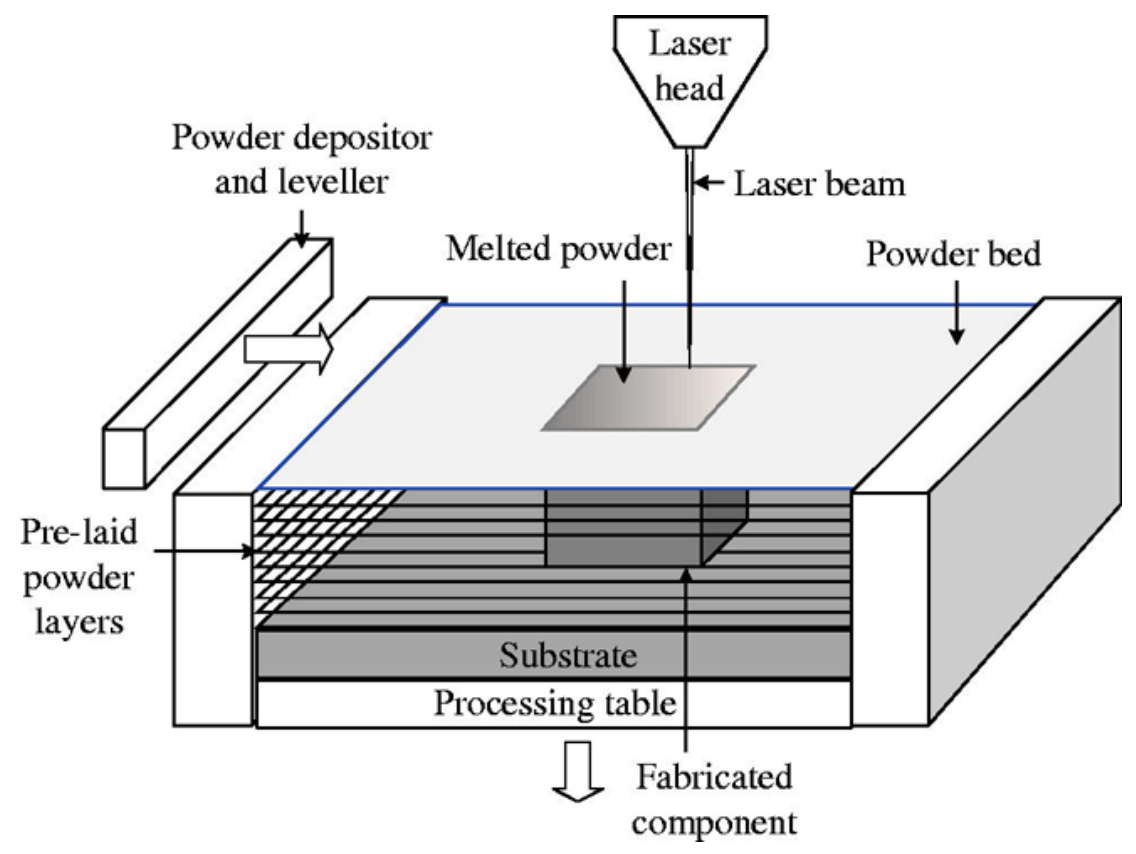

Figure 33 Schematic Diagram of the SLM process [119].

\subsection{Governing equations and boundary conditions}

The finite element formulation [120] for heat transfer in this work is presented in this section. Heat transfer in a material with isotropic thermal properties has the following governing equation,

$$
-\left(\frac{\partial q_{x}}{\partial x}+\frac{\partial q_{y}}{\partial y}+\frac{\partial q_{z}}{\partial z}\right)+Q=\rho c \frac{\partial T}{\partial t}
$$

where $\mathrm{q}_{\mathrm{x}}, \mathrm{q}_{\mathrm{y}}, \mathrm{q}_{\mathrm{z}}$ are components of heat flow through a unit area. According to Fourier's law

$$
\begin{aligned}
& \mathrm{q}_{\mathrm{x}}=-\mathrm{k} \frac{\partial \mathrm{T}}{\partial \mathrm{x}} \\
& q_{x}=-k \frac{\partial T}{\partial x}
\end{aligned}
$$




$$
q_{z}=-k \frac{\partial T}{\partial z}
$$

where:

$\mathrm{K}=$ thermal conductivity coefficient of media.

$\mathrm{Q}=\mathrm{Q}(\mathrm{x}, \mathrm{y}, \mathrm{x})$ inner heat generation rate per unit volume.

$\rho=$ material density

$c=$ heat capacity

\section{Boundary Conditions:}

Specified Temperature: $T_{\mathrm{s}}=\mathrm{T}_{1}(\mathrm{x}, \mathrm{y}, \mathrm{x})$ on area $\mathrm{S}_{1}$.

Specified Heat Flow: $q_{x} n_{x}+q_{y} n_{y}+q_{z} n_{z}=-q_{s}$

\section{Element Formulation:}

Temperature distribution inside an element is approximated using shape functions.

$$
\begin{gathered}
\mathrm{T}=[\mathrm{N}]\{\mathrm{T}\} \\
{[N]=\left[\begin{array}{lllll}
N_{1} & N_{2} & N_{3} & \ldots . . & N_{n}
\end{array}\right]} \\
\mathrm{T}=\left\{\begin{array}{lllll}
\mathrm{T}_{1} & \mathrm{~T}_{2} & \mathrm{~T}_{3} & \ldots & \mathrm{T}_{8}
\end{array}\right\}
\end{gathered}
$$

Where $\mathrm{T}_{\mathrm{i}}=$ temperature at the ith node of the element.

Temperature gradient can be written as 


$$
\left\{\begin{array}{l}
\frac{\partial \mathrm{T}}{\partial \mathrm{x}} \\
\frac{\partial \mathrm{T}}{\partial \mathrm{y}} \\
\frac{\partial \mathrm{T}}{\partial \mathrm{y}}
\end{array}\right\}=\left[\begin{array}{ccc}
\frac{\partial \mathrm{N}_{1}}{\partial \mathrm{x}} & \frac{\partial \mathrm{N}_{2}}{\partial \mathrm{x}} & \ldots \\
\frac{\partial \mathrm{N}_{1}}{\partial \mathrm{y}} & \frac{\partial \mathrm{N}_{2}}{\partial \mathrm{y}} & \ldots \\
\frac{\partial \mathrm{N}_{1}}{\partial \mathrm{z}} & \frac{\partial \mathrm{N}_{2}}{\partial \mathrm{z}} & \ldots
\end{array}\right]\{\mathrm{T}\}
$$

\section{Global FEM Equations:}

The global linear FEM equation for the transient linear problem is:

$$
[\mathrm{C}]\{\dot{\mathrm{T}}\}+\left[\mathrm{K}_{\mathrm{C}}\right]\{\mathrm{T}\}=\left\{\mathrm{R}_{\mathrm{Q}}\right\}
$$

where

$$
\begin{gathered}
C=\int_{V} \rho N^{T} c N d V \\
{\left[K_{c}\right]=\int_{V} B^{T} k B d V} \\
R_{Q}=\int_{V} Q N^{T} d V
\end{gathered}
$$

$\dot{\mathrm{T}}$ is a nodal vector of temperature derivatives with respect to time.

\section{Integration of Transient Thermal problem:}

The Crank Nicolson integration scheme [121] or generalized trapezoidal rule is used to integrate equation (69). This rule is:

$$
\left\{T_{n+1}\right\}=\left\{T_{n}\right\}+\Delta t\left\{(1-\beta)\left\{\dot{T}_{n}\right\}+\beta\left\{\dot{T}_{n+1}\right\}\right\}
$$

Where

$\beta=\frac{1}{2}$ is a integration parameter for the Crank Nicolson integration scheme $\Delta \mathrm{t}=\mathrm{t}_{\mathrm{n}+1}-\mathrm{t}_{\mathrm{n}}$ 
$\mathrm{T}_{\mathrm{n}}=$ known nodal temperature at time $\mathrm{t}_{\mathrm{n}}$

$T_{n+1}=$ temperature at time $t_{n+1}$

Substituting equation (75) into above equation (77) gives following equation:

$$
\left(\frac{1}{\beta \Delta t}[C]+[K]\right)\left\{T_{n+1}\right\}=R_{Q}+[C]\left(\frac{1}{\beta \Delta t}\left\{T_{n}\right\}+\frac{1-\beta}{\beta}\left\{f_{n+1}\right\}\right)
$$

\subsection{Phenomenological Multi-scale Formulation}

The SLM scan pattern generally has a block pattern or continuous scan pattern where the geometry is divided into square blocks and each block is scanned subsequently with serpentine or helical patterns. A representative scan pattern is shown in figure 34 .

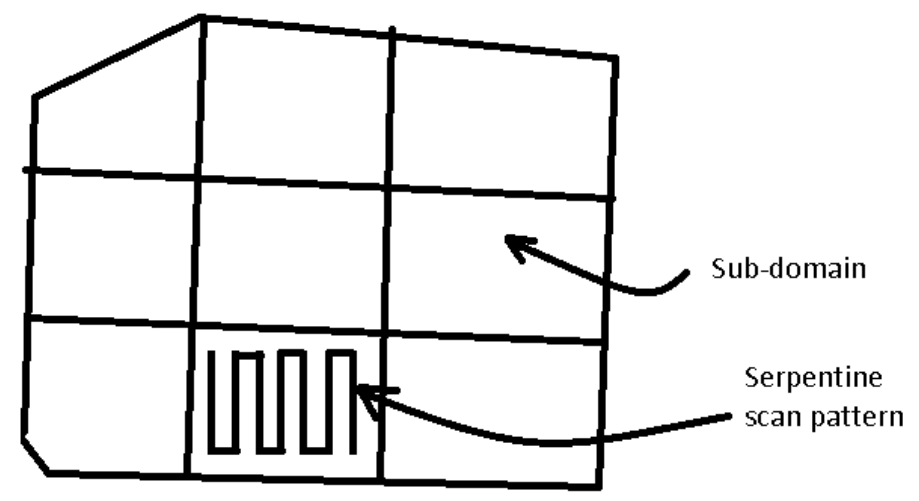

Figure 34 Scan pattern used in SLM

The temperature distributions away from the melt pool have much smaller gradients in time and space than near the melt pool. This is a multi-scale behavior where near the high gradient melt pool region, a higher mesh density is required compared to the rest of the 
powder bed. In the present work two separate simulations are proposed for full bed temperature variation and the sub-domain region as shown in the figure 35 . These two scales are connected by specifying boundary conditions from one scale to another iteratively. The difference in sub-modeling and the proposed two scale model is that it can consider the coupling between two scales. The two scales are described separately in the following discussion.

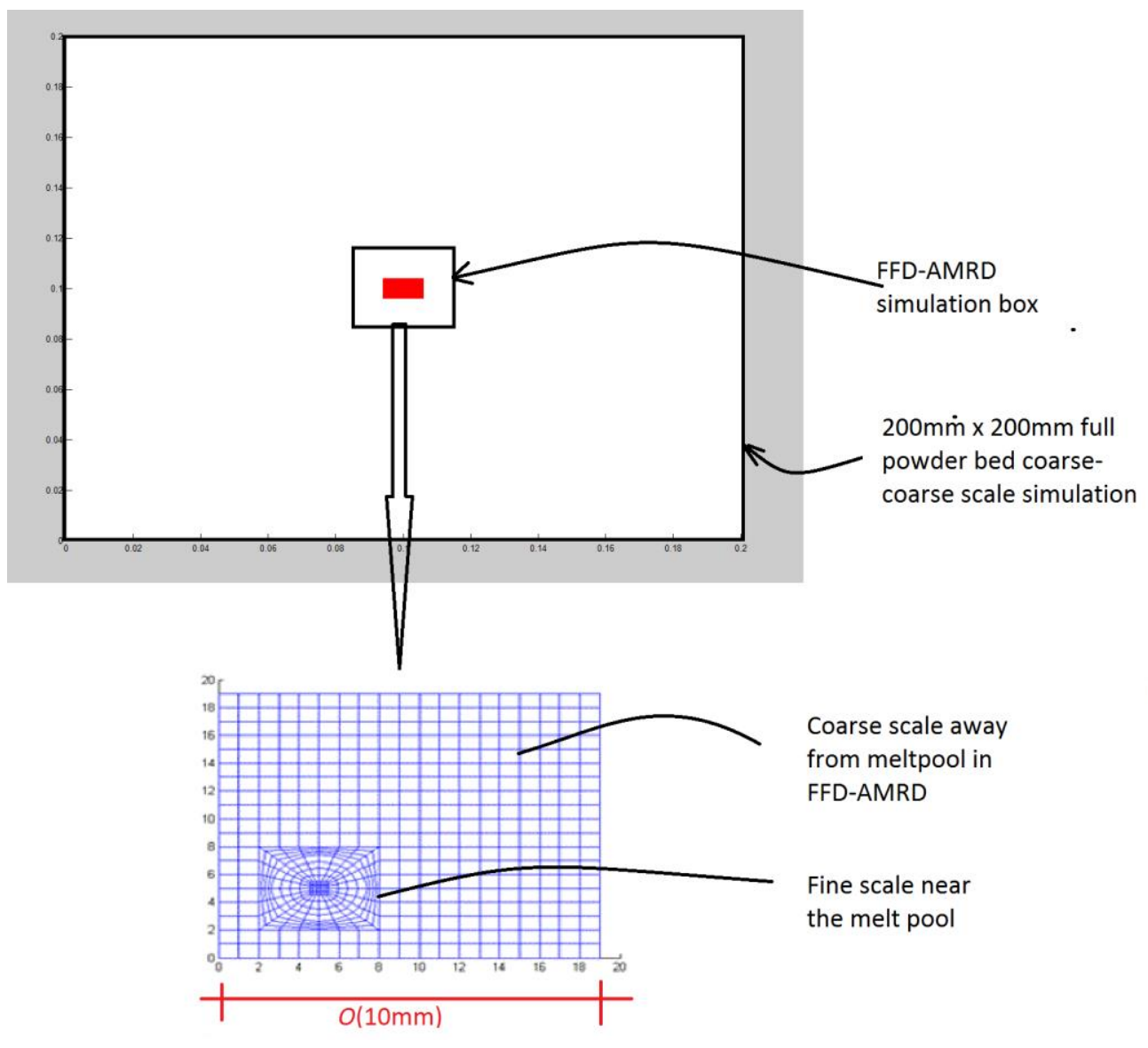

Figure 35 Multi-scale analysis framework for simulation of the SLM process 


\subsubsection{FFD-AMRD simulation covering fine-scale and fine to coarse scales:}

The detailed description of the FFD-AMRD simulation software and its application to SLM were previously described in the literature [117,118]. This model covers simulation of serpentine scans in one sub-domain based on the boundary conditions provided by the coarse scale model. This model further considers various nonlinearities involved such as temperature dependent thermal material properties.

This novel numerical tool has the capability to address multi-scale interactions involved in localized spatiotemporal behaviors, for example the thermal response in case of a moving weld spot or localized deformation in sheet metal rolling processes. Traditional finite element simulations require a very fine refinement to follow the moving localized spatiotemporal response with very high spatial and temporal derivatives. This can be attributed to the fact that the scale of the localized behavior could be $10^{n}$ smaller compared to the macroscopic scale response. These two scales are efficiently bridged with FFDAMRD software. This software has been compared to the best available commercial software and it is found to be 10 to 20 times faster and more memory efficient. The features that help this approach achieve this time and memory efficiency are intelligent element and node renumbering, efficient data transfer between different mesh domains and mesh reassembly techniques.

FFD-AMRD has the ability to model a general hatch pattern read from input files. The temperature dependent thermal properties and density data are read from input files. One of the challenges in the simulation of the SLM process had been simulation of temperature 
dependent material properties (for example latent heat of fusion and vaporization). In the present software these phenomena are taken care of with mathematically rigorous numerical formulations to avoid instability in time integration. The boundary conditions taken into consideration are surface convection and constant temperature boundary conditions at the base plate or on the sides, and radiation if required. The record of simulated thermal history over time leads to evolution of the solidified part geometry and phase information in the solidified part, un-melted powder that has seen a phase change, and virgin powder in its parent phase. These outputs can help when optimizing machine input parameters to achieve better cooling rates and microstructures, resulting in better part life with improved mechanical properties.

This model will be used to calculate total heat input in terms of the external flux from a laser beam and the heat absorbed by the material while changing phases, including the latent heat of vaporization and fusion.

\subsubsection{Macro scale simulation}

This simulation framework will deal with global temperature variations in the powder bed and will give temperature or flux boundary conditions to the FFD-AMRD simulation. This simulation will give the initial temperature of the subdomain and its boundary temperature as function of time. The amount of flux and its distribution as a boundary condition depends on the various energy consumptive nonlinear mechanisms involved at the fine scale behavior inside and near the melt pool. 


\subsubsection{Interaction algorithm between two scale models}

The two scales are connected by the amount of heat flux transferred from the finer scale to the macroscopic scale. Flux quantification is important from the perspective of amount of heat lost or gained in the material state or phase changes. The boundary conditions and initial temperatures given to the fine scale are a function of macroscopic model thermal evolution. The coupling and information transfer between two models is shown in figure 36.

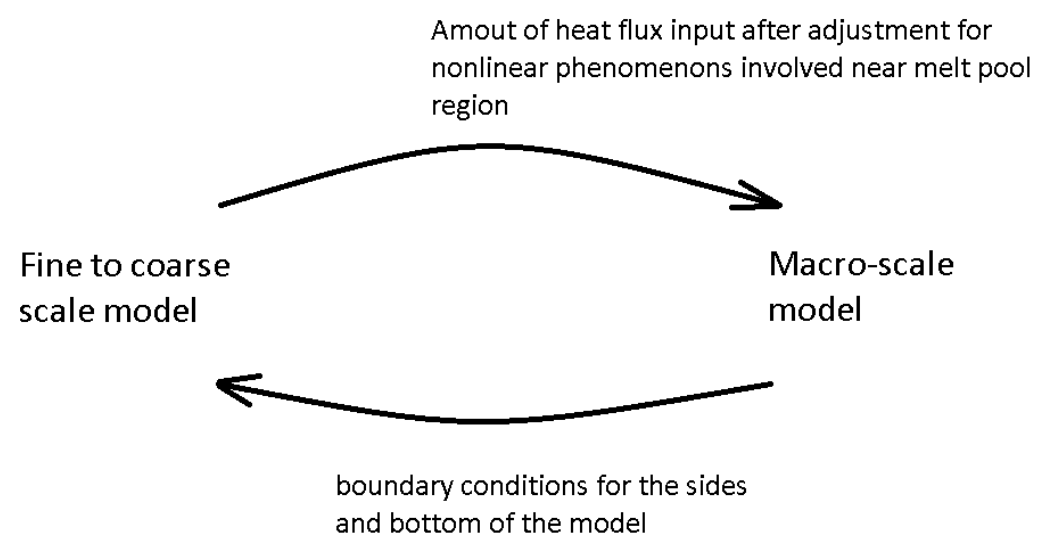

Figure 36 Interaction between two scale models

\subsection{Case Study for Coupling Evaluation between Two Separate Scale Models}

The coupling between two different scales is evaluated in this section with emphasis on the history dependence of the process. The history dependence is mainly due to the slowly evolving macroscopic thermal modes in the macro scale model. In the present work, only the effects of macro scale model temperatures on the fine model are evaluated. The vice versa effects of fine scale thermal effects on the macro scale model are saved for future work. 
The methodology adopted for this purpose is to give different initial macro scale temperatures in FFD-AMRD simulations and find their effect on various variables of interest. The macro scale temperatures have slow variations compared to fine scale thermal behavior. This slow evolution can be exploited to simplify the analysis as well as to evaluate the coupling between two models. The assumption here is that the macroscopic thermal distribution has an almost flat distribution at the lower scale. The material properties [122] of the stainless steel powder considered for this simulation are taken from literature.

\subsubsection{Melt pool Diameter}
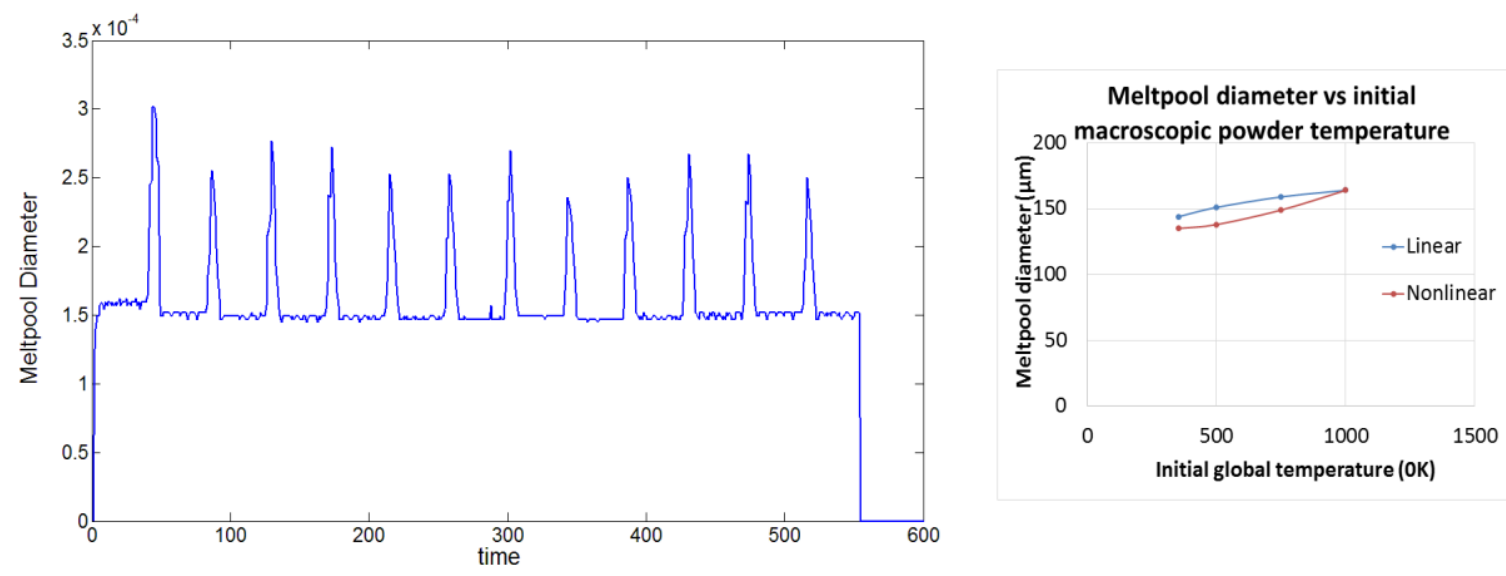

Figure 37 : (a) Melt pool diameter variation during a subdomain hatch for the case with $750 \mathrm{~K}$ initial temperature. (b) Effect of initial global temperature on melt pool in SLM of stainless steel powder.

Figure 37 shows the variation of the melt pool diameter in the $\mathrm{Y}$ direction. The melt pool diameter in the case of linear simulations is higher at lower preheating temperatures 
compared to the melt pool diameter in the nonlinear simulation. The reason behind the diameter decrease of about $30 \mu \mathrm{m}$ for a $650 \mathrm{~K}$ initial powder bed temperature for nonlinear simulation is due to the increased heat loss by heat of vaporization and fusion. These diameters are measured at the center of the scan hatch where melt pool diameters and profile are stabilized.

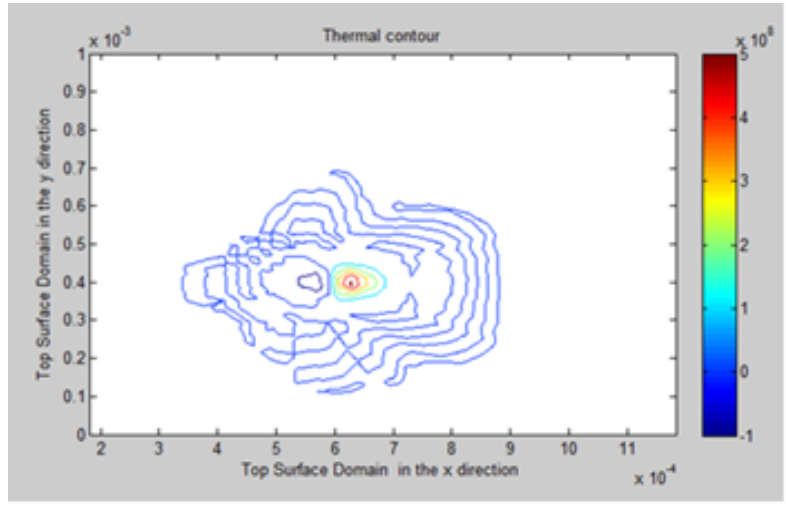

(a) $500 \mathrm{~K}$ initial temperature

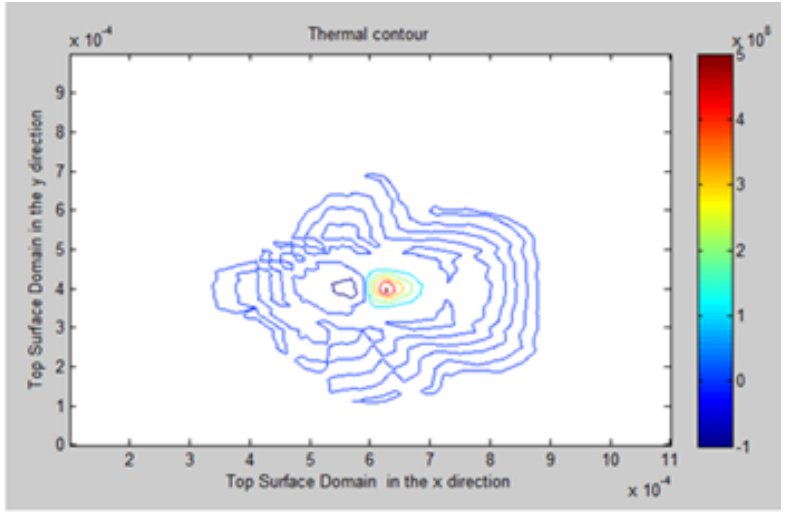

(b) $400 \mathrm{~K}$ initial temperature

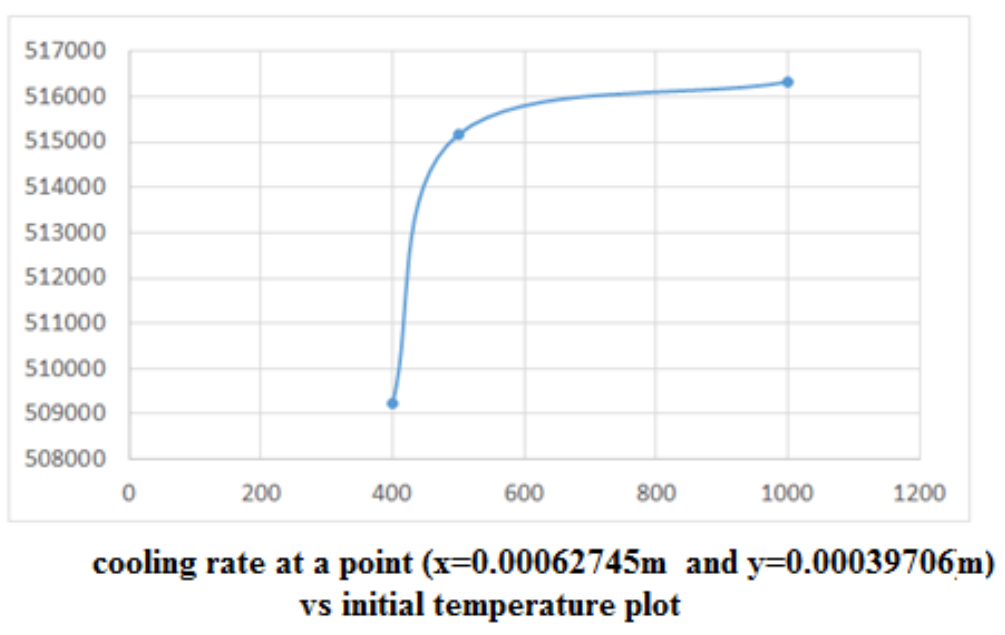

Figure 38 Cooling rates at different initial powder bed temperatures

\subsubsection{Cooling Rates}

Two sample cooling rate plots are shown in figure 38 ( $a$ and $b$ ) for powder initial temperature of $500 \mathrm{~K}$ and $400 \mathrm{~K}$. The cooling rates in both cases are not significantly 
different than $0.1 \%$ as it can be seen in the figure 38 (c). The effect of global heating is seen to be not very significant on the cooling rates. This leads to the conclusion that cooling rate is not a significantly coupled function of macroscopic temperature. In order to control the cooling rates, local laser characteristics such as laser power and scan speed have greater influence.

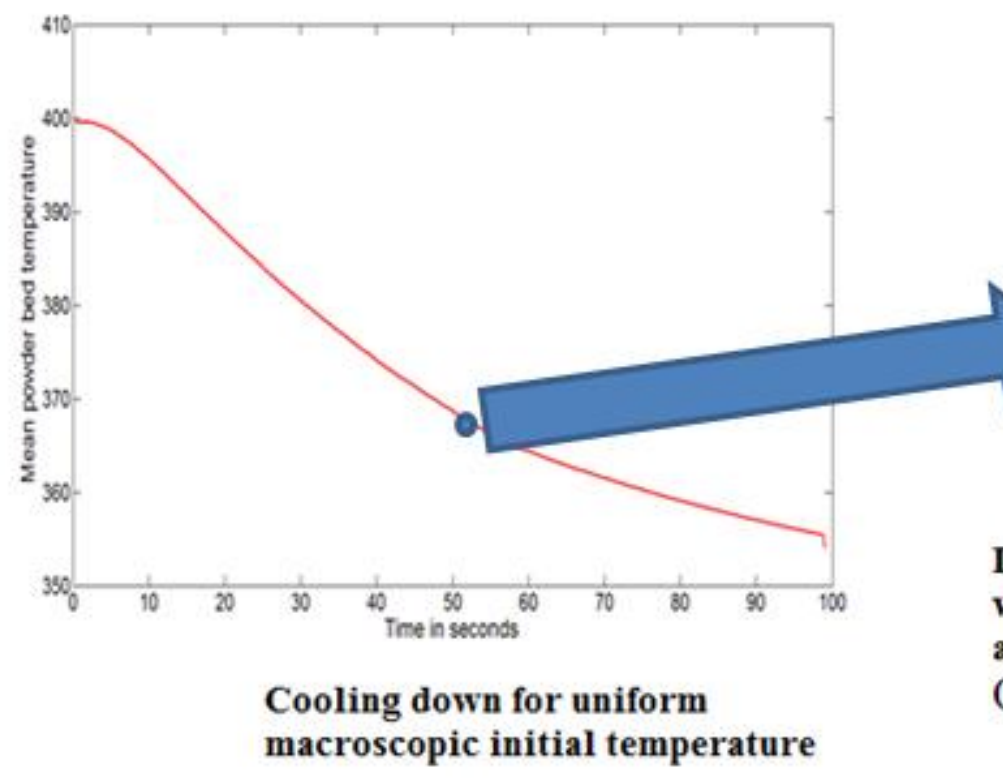

(a)

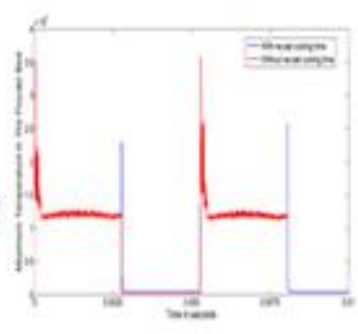

Local temperature variations with fast temporal scale activated by the laser beam (refer Figure 41 for details)

(b)

Figure 39 Powder bed cooling down through global mode of uniform average temperature. The zoomed in picture shows the local fast temporal variation of temperature due to laser beam inside fine to coarse model.

\subsubsection{Macroscopic powder bed cooling rates}

The powder bed of size $5 \mathrm{~mm}$ square is allowed to cool down from initial temperature of $400 \mathrm{~K}$. The purpose of this experiment is to compare cooling rates of the macroscopic scale compared to local thermal modes near melt pool thermal variations. Figure 39 shows 
a schematic concept and the actual quantitative results obtained through the numerical experiment.

The temperature variations across the full powder bed are seen to be changing very slowly as shown in figure 39 (a) or have low frequencies. Whereas the local modes (figure 39 (b)) activated due to the laser beam flux have high frequency and that makes them heat up very fast and cool down at a similarly fast rate. The local modes have the major contribution towards the near Gaussian temperature distribution in the melt pool. The global modes are associated with the full powder bed temperature evolution over time.

\subsection{Effect of subdomain scan pattern on thermal history}

To determine the effect of scan pattern and the time required to cool down the powder around the last melt pool, a numerical experiment has been attempted. This study explores the importance of hatch pattern for successive subdomains on the thermal history. Figure 40 (a) shows the simulated scan pattern. The subdomain on top is hatched first and then the subdomain at the bottom is hatched. The influence of first subdomain hatch is evaluated indirectly by allowing the powder bed a small time to cool down after its hatch is completed. The cool down will reduce the last melt pool spot temperatures towards the global average. This will indirectly quantify if there was any significant influence of prior neighboring subdomain scan. If significant cooling time is allowed then the effect of last melt pool spot will be almost nullified. 
The maximum temperature at a particular time instance is shown in figure 41 . In order to compare corresponding temperatures in two cases with respect to laser position, a plot with no cooling time is given a blank temperature offset from 0.00253 to 0.0053 second. This is to account for the difference in total simulation times between two cases. A cool down time of $0.0025 \mathrm{~s}$ is given between successive scans.

The effect of heating of the powder due to a previous nearby scan is seen to be almost negligible as the maximum and average temperature of the bed has identical variation in both cases. The bed almost cools down in a distance of $1 \mathrm{~mm}$ to the global powder bed temperature as shown in figure 40(b). This fast cooling down of the melt pool trace in the prior subdomain hatch leads to negligible thermal "cross-talk" in nearby subdomain hatches. The cooling times for the melt pool is approximately $0.0005 \mathrm{~s}$ as shown in figure 41.

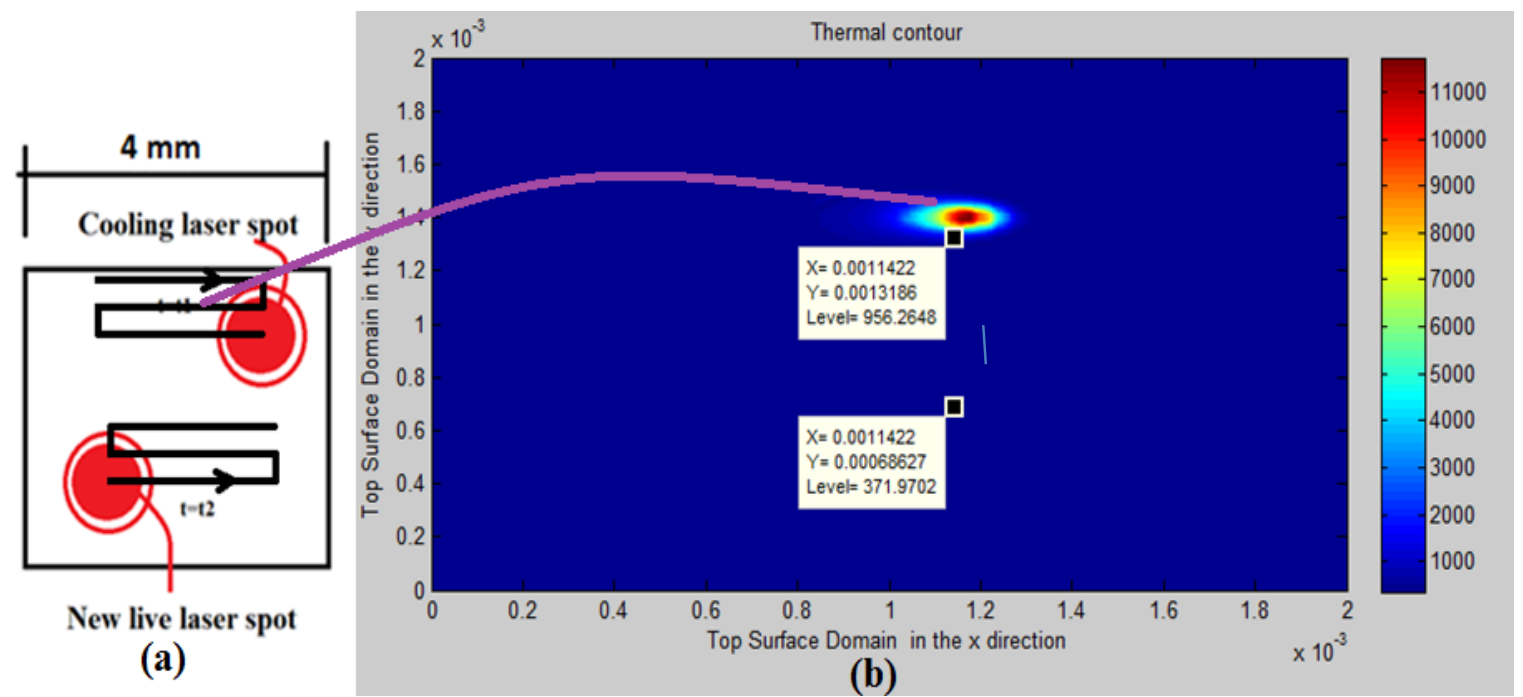

Figure 40 (a) Scan pattern used for two successive subdomains scan (b) Thermal profile at particular time in the prior subdomain scan. 


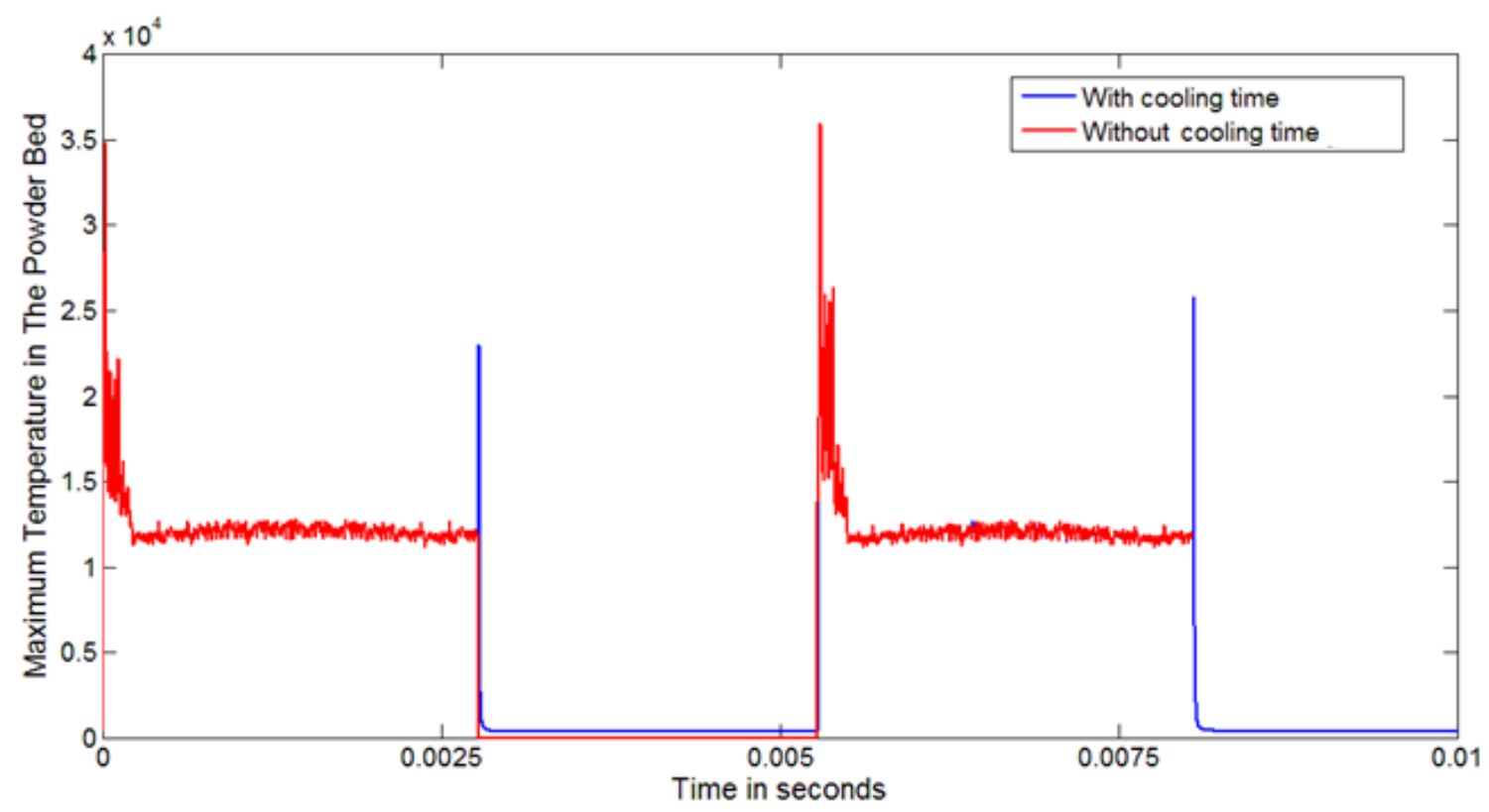

Figure 41 Maximum Temperature comparison in two cases (with and without cooling time between successive scans)

\subsection{Beam Theories and Dimensional Reduction}

Beam theories were developed over a long period of time, starting with the Euler Bernoulli beam theory in 1750 . Over time beam theories have evolved into more and more accurate formulations. Traditional beam theories have limitations for complicated geometries, in which case ad-hoc assumptions in the beam theories lead to incorrect structural response predictions.

A recent development in beam theories is the Variational Asymptotic Method [123] (VAM) for dimensional reduction. Dimensional reduction is a term used to describe a generalized physics based beam theory used for solving partial differential equations involved in various physical phenomena. The main limitation of VAM is the energy based 
formulation which limits its applications in problems involving nonlinear energy dissipative phenomena like plasticity. VAM starts with an order of magnitude analysis and order of each term energy expression, which is stress multiplied by strain. Each term is evaluated for the order of the magnitude. This helps in solving the equilibrium equation at different orders of magnitude and a solution at each order gives a particular mode of response in the dimensional reduction.

The accuracy of VAM solutions to Partial Differential Equations is limited by the fact that the order of magnitude analysis performed can be limited to a few global macroscopic modes which need not be orthogonal, particularly in the case of highly inhomogeneous cross-sections. This limitation can be attributed to the fact that in the case of inhomogeneous domains it is hard to predict variations based on superficial dimensions (order of magnitude analysis based on geometric small aspect ratio) of the prismatic structure. The local stress or strain concentrations for generalized cross-sectional geometries are hard to account for using this strategy.

\subsubsection{Dimensional Reduction for a Prismatic Powder Bed}

In the present case study a simple square cross-section with 10 times more conductive material on one side of the cross-section compared to the bulk as shown in figure 42 is considered. In order to simplify the case study a steady state problem is assumed though 
the mathematical formulation can be extended easily to unsteady problems specifically for SLM simulations.

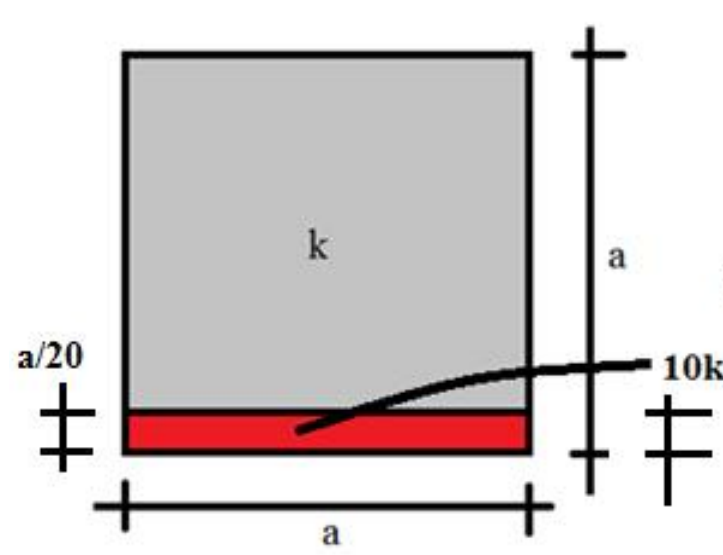

X-Y plane cross-section

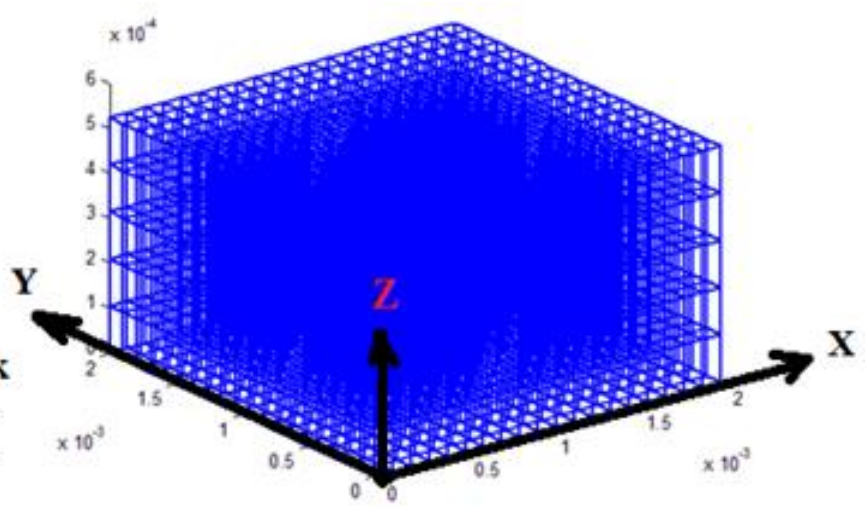

FEM mesh

Figure 42 Figure 10: Problem geometry and FEM mesh

\subsubsection{Modal Space based Beam Theories Formulation}

The stiffness matrix for the FEM discretization described in figure 42 will have a particular pattern of sparsity. It can be described as shown below with $A_{i}$ being a square matrix with a size corresponding to the degrees of freedom in one particular cross-section.

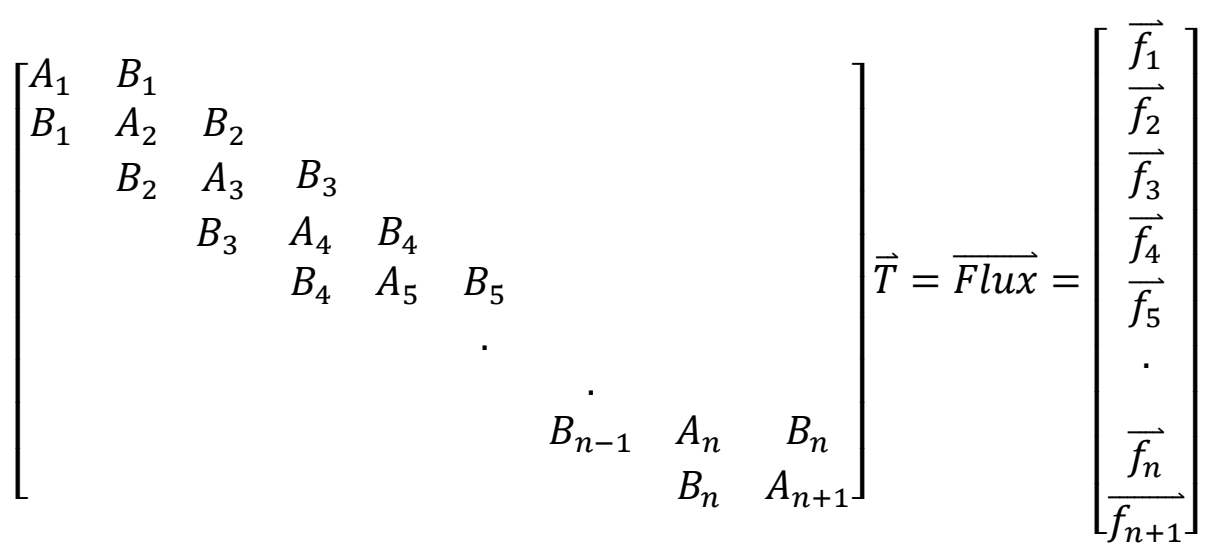


In the case of perfectly prismatic geometry and material like a perfect beam, $A_{i}$ and $B_{i}$ will follow the following relation except at the free end.

$$
\begin{gathered}
A_{i}=A \\
B_{i}=B \\
A_{1}=A / 2 \text { (Free edge stiffness) }
\end{gathered}
$$

Permutations to make the matrix upper triangle will be

$$
\begin{gathered}
P_{1}=B(A / 2)^{-1} \\
P_{2}=B\left(A-P_{1} B\right)^{-1} \\
P_{i}=B\left(A-P_{i-1} B\right)^{-1}
\end{gathered}
$$

These permutations will be operated on both sides of the equation and will create a forward wave in the right hand side and an upper triangulation on the left hand side.

$$
\vec{f}_{i+1}=\vec{f}_{i+1}-P_{i} \vec{f}_{i}
$$

These equations have no closed form explicit solution or any straight forward modal space solution. This difficulty can be overcome by pre-multiplying the whole equation by $B^{-1}$. The permutations for the modified system of equations will look like 


$$
\begin{gathered}
P m_{1}=\left(B^{-1} A / 2\right)^{-1} \\
P m_{2}=\left(B^{-1} A-P_{1}\right)^{-1} \\
P m_{i}=\left(B^{-1} A-P_{i-1}\right)^{-1}
\end{gathered}
$$

This series of permutations have the same modes for any $P m_{i}$. These modes are the same as of the modes of $B^{-1} A$. This is based on the property of Eigen modes that is

- The inverse of a matrix has the same Eigen Modes as that of the original matrix

- Linear equations of matrices with the same vectors have the same Eigen Vectors but different eigenvalues.

Writing $P m_{i}$ and $B^{-1} A$ in the form of modes will lead to following equations.

$$
\begin{gathered}
P m_{i}=\emptyset^{T} D_{i} \emptyset \\
B^{-1} A=\emptyset^{T} K \emptyset \\
P m_{i+1}=\emptyset^{T} D_{i+1} \emptyset \\
\emptyset^{T} D_{i+1} \emptyset=\left(\emptyset^{T} K \emptyset-\emptyset^{T} D_{i} \emptyset\right)^{-1} \\
\emptyset^{T} D_{i+1} \emptyset=\left(\emptyset^{T}\left(K-D_{i}\right) \emptyset\right)^{-1} \\
D_{i+1}=\left(K-D_{i}\right)^{-1}
\end{gathered}
$$

The last equation describes the evolution of Eigen values for the cross-section modes. 


\subsubsection{Converged Eigen values in the bulk}

The Eigen values will converge asymptotically at some particular value as the permutation wave moves towards the bulk. Converged Eigen values will have the following governing equation.

$$
\begin{gathered}
D_{c o v}=\left(K-D_{c o v}\right)^{-1} \\
D_{c o v}{ }^{2}-K D_{c o v}+I=[0]
\end{gathered}
$$

This equation has an explicit solution because each matrix in it is diagonal.
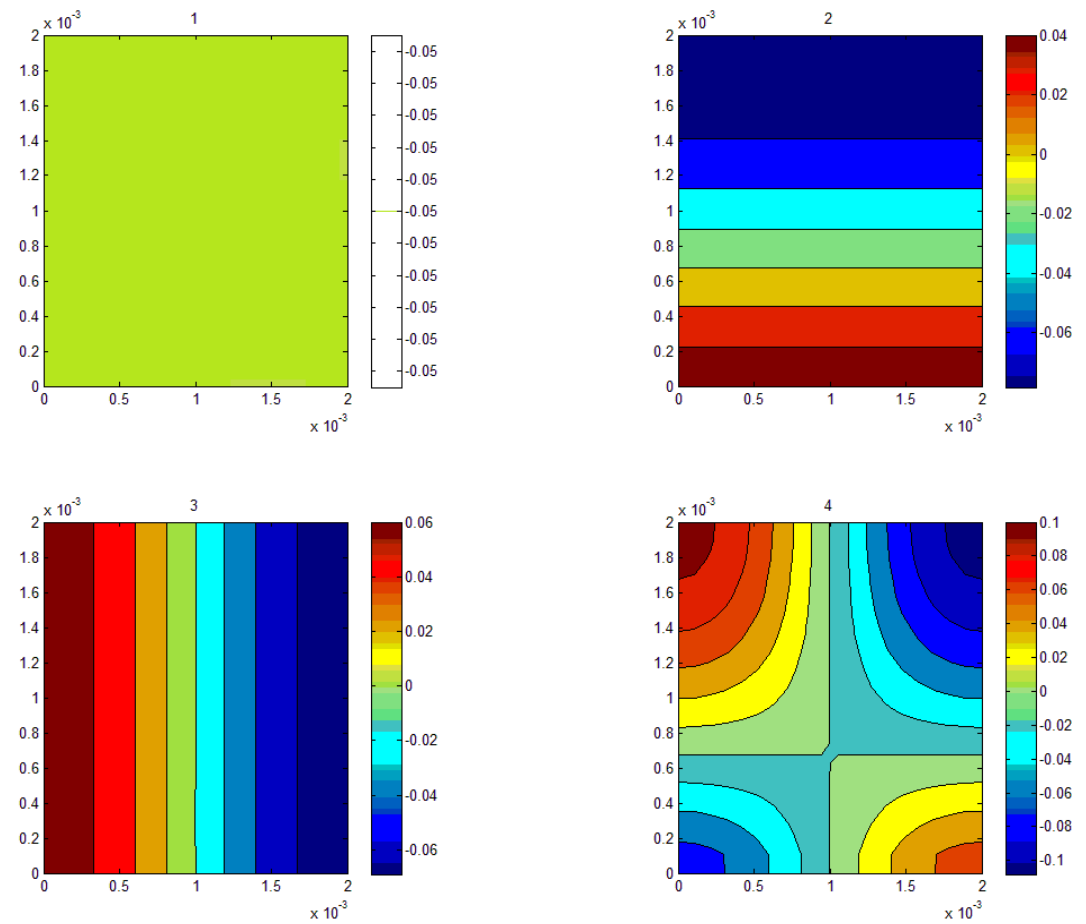

Figure 43 First four significant modes for of the powder bed case study c/s 

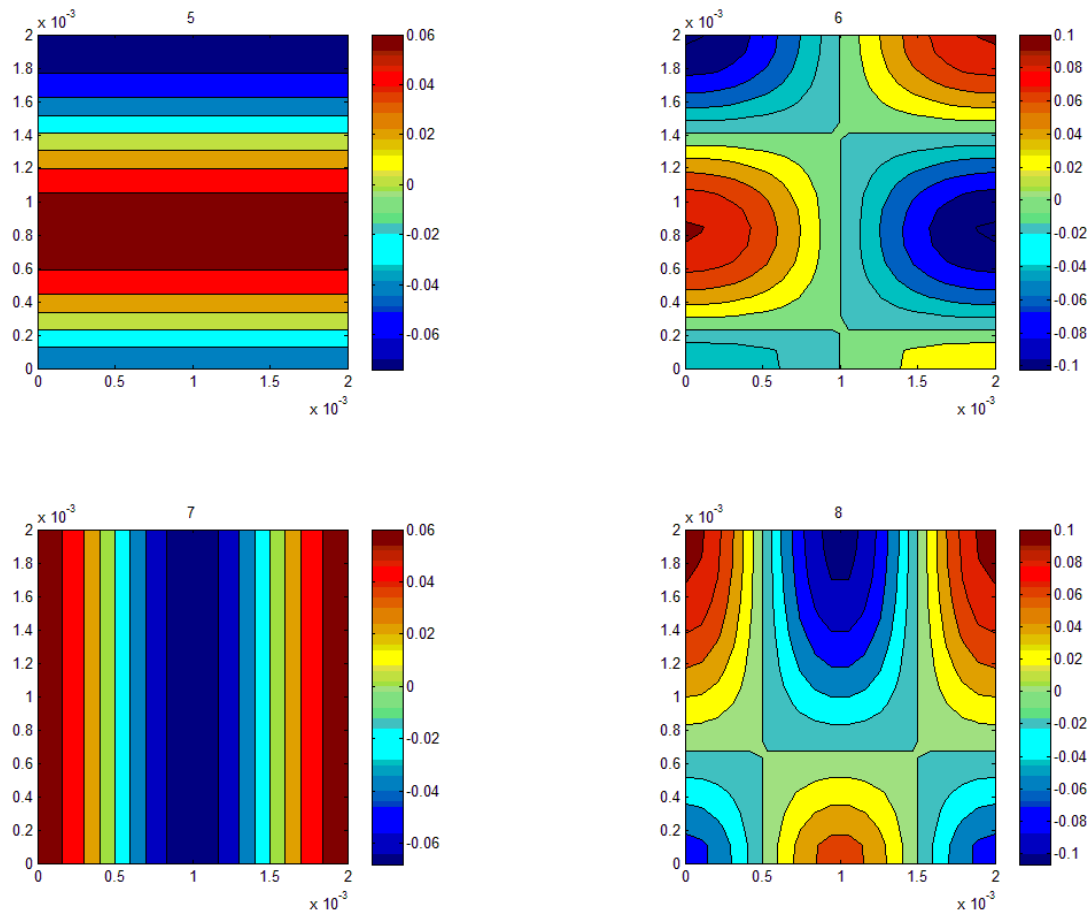

Figure $445^{\text {th }}$ to $8^{\text {th }}$ significant modes for of the powder bed case study $\mathrm{c} / \mathrm{s}$

$$
D_{c o v}=\frac{\left(K \pm \sqrt{K^{2}-4}\right)}{2}
$$

This proves that the minimum Eigen value for a real solution is equal to 1 . This is also supported by the physics of the problem.

\subsubsection{Eigen Modes for the prismatic powder bed: case study}

The Eigen modes $\emptyset^{T}$ of the prismatic geometry shown at the start of this section are derived based on the above formulation. The first 8 significant modes are shown in figures 43 and 44. The first mode has an Eigen value equal to 1.00 . 


\subsection{Extension of the present mathematical formulation to geometry with dissimilar layers}

The assumption in the above formulation was that successive layers have material property variation across a cross-section. This assumption can be easily removed by evaluation of coupling between successive layers. Considering the number of modes that are of interest and the weak coupling between distant modes in the spectrum leads to small sized and sparse coupling matrices which need to be solved.

\subsection{Conclusions}

- Two separate scales of models for simulation of the SLM process are proposed in order to reduce computational complexity involved in simulations.

- It is seen in the numerical experiments that the two scale model can help in developing simple laws for various parameters of interest near the melt pool region. These experiments also give a phenomenological understanding of the coupling between two scales.

- The new mathematical formulation for dimensional reduction developed in this paper has significant potential for reducing the computational complexity involved in simulations involving large number of completed build layers in the analysis.

The phenomenological understanding developed through this work is as following.

1. Cooling rates are not sensitive to the global mode preheating temperatures or initial temperatures. This also means that the cooling rates can be controlled only through laser power control. 
2. The melt pool spot cool down time to near average bed temperatures is approximately found to be $0.0005 \mathrm{~s}$.

3. Local modes can be more efficiently influenced by energy input at the local level (which will have fast temporal scale and high frequencies). This provides an opportunity for localized preheating or post heating in order to control cooling rates or residual stresses.

4. Meltpool diameters in the case of nonlinear thermal simulation are lower compared to the diameters simulated through linear thermal simulations. 


\section{CHAPTER 6}

\section{INTELLIGENT CHOLESKY DECOMPOSITION FOR}

\section{EXCLUDING THE COMPUTATIONAL OVERHEAD OF}

\section{INSIGNIFICANT MATRIX MULTIPLICATIONS}

\subsection{Introduction}

The Finite Element Method (FEM) is one of the most well-known numerical techniques used for solving partial differential equations involved in simulation of many types of physical phenomena at a continuum scale. A numerical construct for Gauss point integration used during the determination of unbalanced forces makes this technique good for solving nonlinear thermomechanical problems. Nonlinear simulations using this technique require frequent and iterative updates of the stiffness matrix. For quasi-static problems, the stiffness matrix in each iteration of a particular time step may have different values but the element connectivity remains the same unless an adaptive scheme is used for simulation. Various algorithms used for solving the corresponding simultaneous linear equations are Super LU [124], Cholesky decomposition [125] or wavefront solvers [126]. In general, solvers can be classified into two classes. The first class is based on stiffness matrix decomposition and the second is based on successive iterations. The iterative solvers work well with the availability of an initially good solution approximation whereas the 
decomposition based solvers are more reasonable when prior simulation history is not available or solutions quickly evolve with time such that iterative solvers result in erroneous or slow convergence.

The decomposition of a parent stiffness matrix results in matrices, such as a lower triangulated matrix during Cholesky Decomposition. This matrix involves significant changes in its sparsity compared to the parent stiffness matrix. In literature, attempts have been made [127] where numbers were either included or ignored in analysis based on their floating point accuracies; but a more generalized or accurate pseudo-asymptotic analysis has not been developed. In the presented work, a new algorithm has been developed to evaluate the significance of numbers and/or numerical operations involved during Cholesky decomposition. In this work, the insignificant values in the corresponding lower triangulated matrix are neglected resulting in increased sparsity. The change in sparsity of this matrix w.r.t. to the parent stiffness matrix results in reduced FLOating Point operationS (FLOPS) required for its computation. The need for an initial lower triangulated Cholesky matrix for optimization of future computations using insignificant value filters can be eliminated if a certain threshold on the neighborhood of the degrees of freedom involved in the problem is prescribed, leading to reduced computations required for computing the Cholesky decomposition.

\subsubsection{Variants of Cholesky Decomposition in Literature}

Modifications to Cholesky [128] has been attempted in past to solve symmetric but not positive definite matrices. Computational complexity of Cholesky decomposition has been reduced with an algorithm named as incomplete Cholesky factorization [129]. This 
algorithm works similar to other preconditioning methodologies used in literature for solving simultaneous equations. Different strategies of Cholesky decomposition with left looking or right looking algorithms [130] have been considered to improve computation speeds. These algorithms are significant from the perspective of multi-rank updates and for improving well known interior point linear programming algorithm by Karmarkar [131]. A study on number rounding and perturbation bounds [132] has been performed to evaluate numerical error in factorization.

\subsection{Modified Cholesky Factorization Procedure}

The Cholesky decomposition has the standard form shown in equation

( 88 ).

$$
A=L L^{T}
$$

Values in L are determined using the formula shown in ( 89 ).

$$
L_{i j}=\frac{1}{L_{j j}}\left(A_{i j}-\sum_{m=1}^{j-1} L_{i m} L_{j m}\right) \quad \text { for } i>j
$$

The additional criterion on $L_{i m} L_{j m}$ multiplication or significance of $L_{i m}$ or $L_{j m}$ helps reduce unnecessary computational FLOPS. This indirectly leads to many addresses in the lower triangulated Cholesky matrix attaining a value equal to zero. Detailed algorithms and various alternate insignificant value filtering criteria are listed in the following subsections. In addition to insignificant value based numerical filters, a conservative neighborhood criterion employed at each nodal degree of freedom in the stiffness matrix has also been used for this purpose. 


\subsubsection{Algorithm}

A general algorithm for the proposed intelligent Cholesky algorithm is provided below. This algorithm is quite general and could be used for most of the criteria to filter insignificant values and numerical operations.

1. Calculate Stiffness matrix for initial material properties.

2. Decide an approximate initial tolerance based on existing values in the stiffness matrix.

3. Use the algorithm provided in Figure 45: Algorithm to determine which values in the E matrix have information which are significant to calculate the $\mathrm{E}$ matrix. Use different criteria listed in the next section to compute each successive value in the Cholesky matrix. A matrix $\mathrm{E}$ will be computed here which has information of which addresses in $L$ are significant to compute in the binary format.

4. Record numerical operations which are insignificant to perform. This will require an efficient way to store and retrieve the information about successive significant or insignificant numerical operations.

5. Record the number of FLOPS required with the tolerance used.

6. Change tolerance and repeat the above-mentioned procedure 1-5 until the error is within an acceptable limit in addition to the total number of FLOPS which is at its optimally lowest value.

7. Use the information in the $\boldsymbol{E}$ matrix and the information stored about significant numerical operations for the next iteration for the same or different stiffness matrices for the same mesh with different material properties in case of any nonlinearity.

\subsubsection{Variations of the present procedure}

Different criteria which can be used to calculate the $\boldsymbol{E}$ matrix and significant numerical operations involved in these computations have been stated below. 
Criterion 1: Calculate the exact Cholesky decomposition and filter numbers for a particular tolerance to determine significant values in the $\boldsymbol{L}$ matrix.

Criterion 2: Neighborhood heuristics where $L_{i m} L_{j m}$ multiplications for a particular value of $m$ in equation (89) are computed only if $m$ is within a particular distance away from non-zero values in the corresponding row of the stiffness matrix. Application of these criteria has been described with an algorithm in Table 3.

Table 3 Algorithm for Criterion 2 for number filtering

$$
\begin{aligned}
& \text { For } \mathbf{i}=\mathbf{1} \text { to DOFs } \\
& \text { For } \mathbf{j}=\mathbf{1} \text { to } \mathbf{i}-\mathbf{1} \\
& \text { For } m=1 \text { to } j-1 \\
& \qquad \begin{array}{c}
\text { Sum }=0 \\
\text { Var }=\text { minimum (index distance between non }- \text { zero value of } i^{\text {th }} \\
\text { If }(\text { Var }<\text { tolerance }) \\
\text { sum }=\text { sum }+L_{\text {im }} L_{j m}
\end{array}
\end{aligned}
$$

\section{End}

\section{End}

$$
L_{i j}=\frac{1}{L_{j j}}\left(A_{i j}-\text { sum }\right) \quad \text { for } i>j
$$

End

$$
L_{i i}=\left(K_{i i}-\sum_{m=1}^{i-1} L_{i m} L_{j m}\right)^{1 / 2} \quad(\text { similar criterions can be used here as well) }
$$

\section{End}

Table 1: Algorithm for Criterion 2 for number filtering 


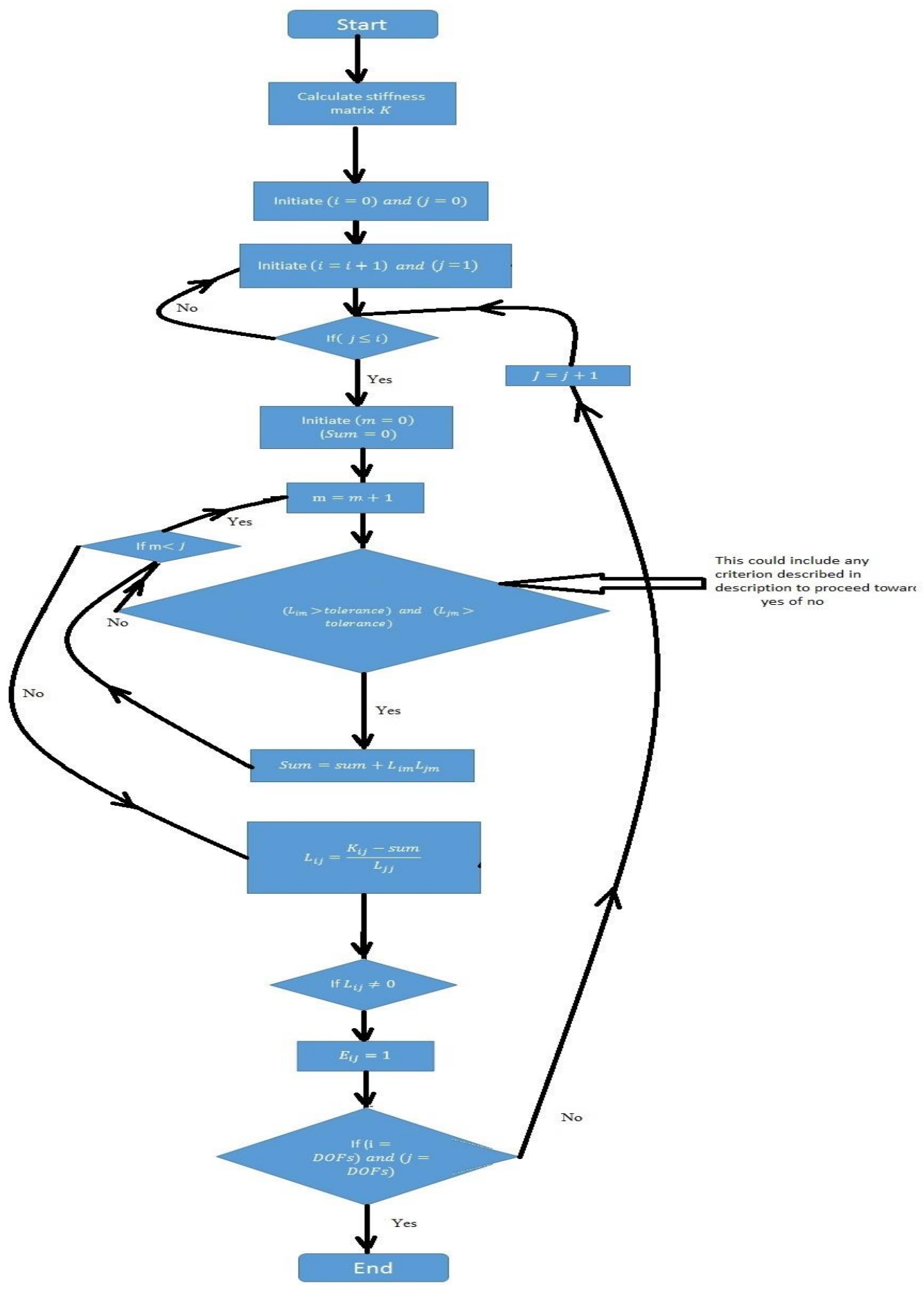

Figure 45: Algorithm to determine which values in the E matrix have information which are significant 
Criterion 3: Multiplication criterion: $L_{i m} L_{j m}$ multiplications in ( 89 )are computed only if the absolute value of the multiplication is above a certain tolerance value. Variation of this criterion includes following the criterion on numerical computations in ( 88 )in order to consider a particular $L_{i m} L_{j m}$ multiplication.

$$
\frac{L_{i m} L_{j m}}{L_{j j}}>\text { tolerance }
$$

Criterion 4: $L_{i m} L_{j m}$ multiplications in equation 1 are computed only if the individual values $L_{i m}$ and $L_{j m}$ are both above a certain tolerance.

Criterion 5: Neighborhood heuristics where $L_{i m} L_{j m}$ multiplications for a particular value of $m$ in (Equation ( 89 ) are computed only if $m$ is within a particular distance from any non-zero values in the $i^{\text {th }}$ or $j^{\text {th }}$ row of the stiffness matrix.

\subsection{Case Study: Steady state thermal analysis with intelligent Cholesky algorithm}

In this study, a prismatic geometry subjected to boundary conditions on two opposite faces in the $\mathrm{z}$ direction is considered. The problem geometry and the boundary conditions are shown in Figure 46: Problem geometry and boundary conditions. The dimensions of the prismatic geometry are provided below. All units used in this case study are in SI units.

$$
\begin{gathered}
x_{-} l=0.0005 \text { unit } \\
y_{l}=0.0005 \text { unit } \\
z_{-} l=\left(1.7241 \times 10^{-5}\right) \text { unit }
\end{gathered}
$$

The constrained boundary condition applied on the bottom surface is as follows: 
$T(x, y)=0$

A uniform flux boundary condition has been applied on the top surface in the $+\mathrm{z}$ direction. The total magnitude of the integrated flux on this surface equals 1 unit. The thermal conductivity has been assumed to be $k=6.7$ units.

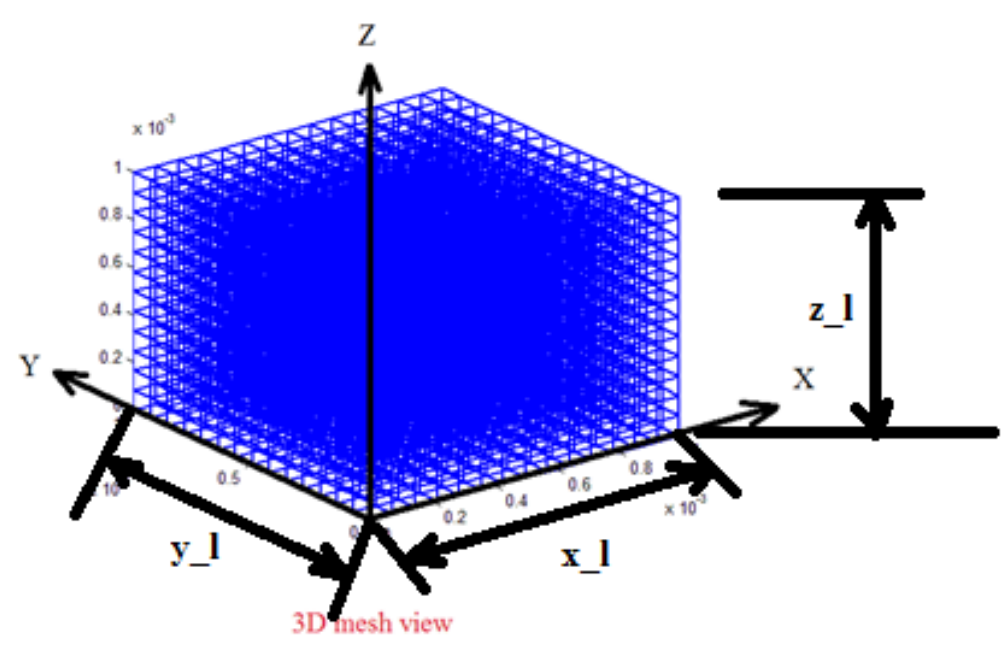

Figure 46: Problem geometry and boundary conditions

The mesh discretizations used in this case study are as follows:

$$
\begin{aligned}
& \text { Xnode }=30 \\
& \text { Ynode }=30 \\
& \text { Znode }=4
\end{aligned}
$$

The problem posed here has been attempted using criteria 2 and 3 as mentioned in section 6.2.2. The results obtained using Matlab Cholesky and intelligent Cholesky algorithms have been presented in the following subsections. 


\subsubsection{Results with Criterion 2}

In this subsection, criterion 2 has been used for the computation of the lower triangulated Cholesky matrix with different tolerance levels between 20 and 160. In Figure 47, the ratio of maximum error at any DOF, number of FLOPS and total non-zero values appearing in the Cholesky matrix in comparison with Standard or Matlab Cholesky algorithms are plotted. In Figure 48 and Figure 49, thermal nodal degrees of freedom are compared between the neighborhood and insignificant value filtering Cholesky decomposition algorithms.

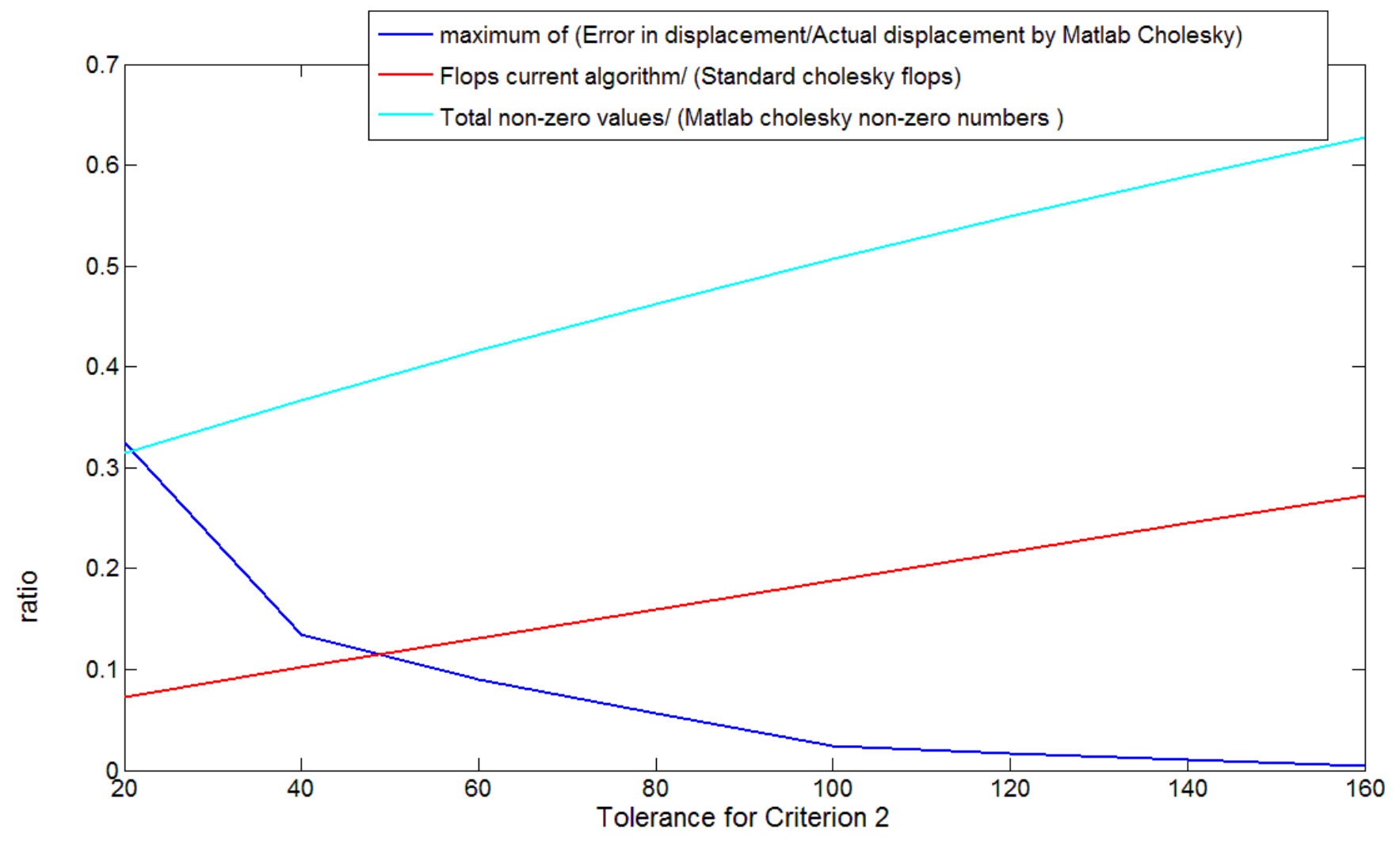

Figure 47: Comparison between standard Cholesky versus Intelligent Cholesky algorithm presented with criterion 2 . 


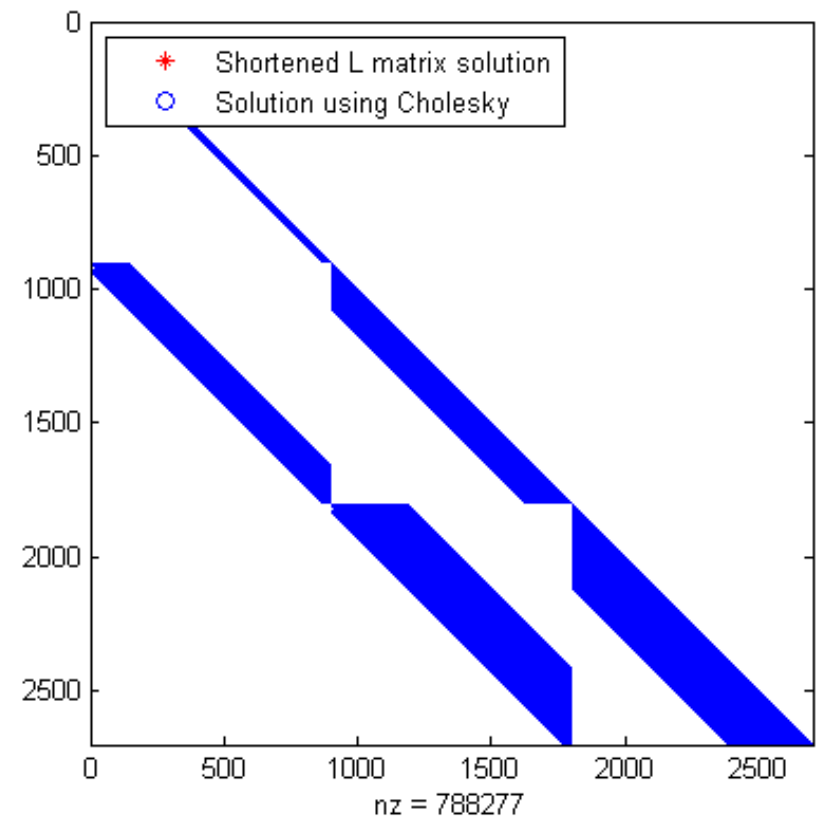

(a)

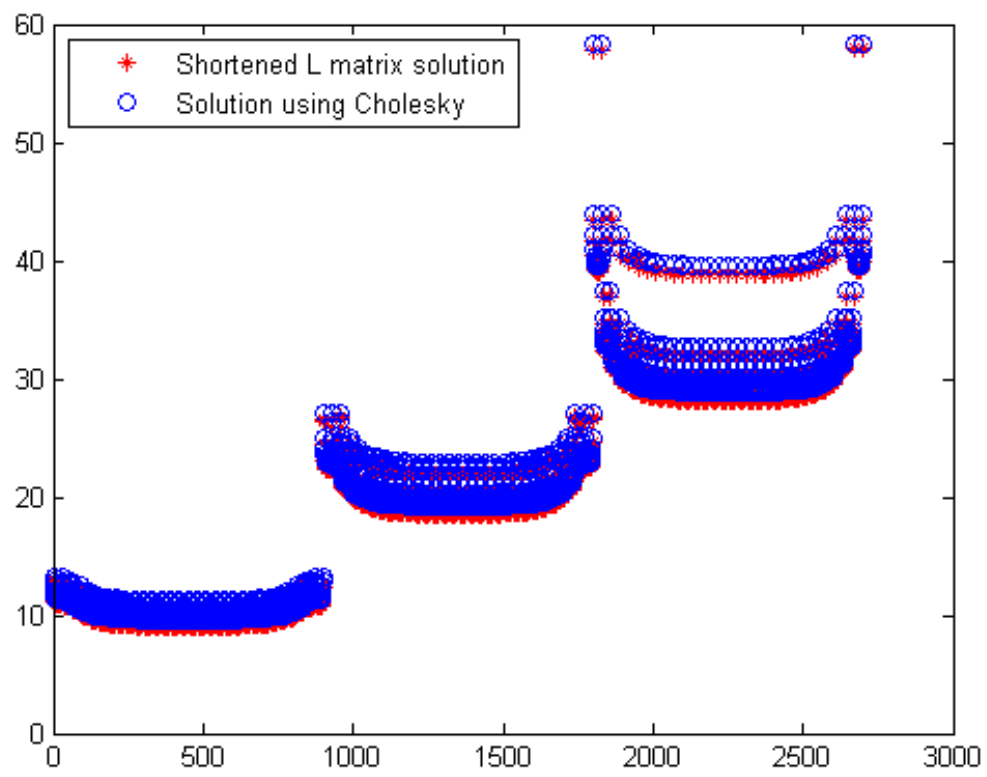

(b)

Figure 48 (a) Cholesky matrix computed through the intelligent Cholesky algorithm. (b) Comparison of temperatures computed using intelligent Cholesky and Matlab Cholesky at a threshold of 80 

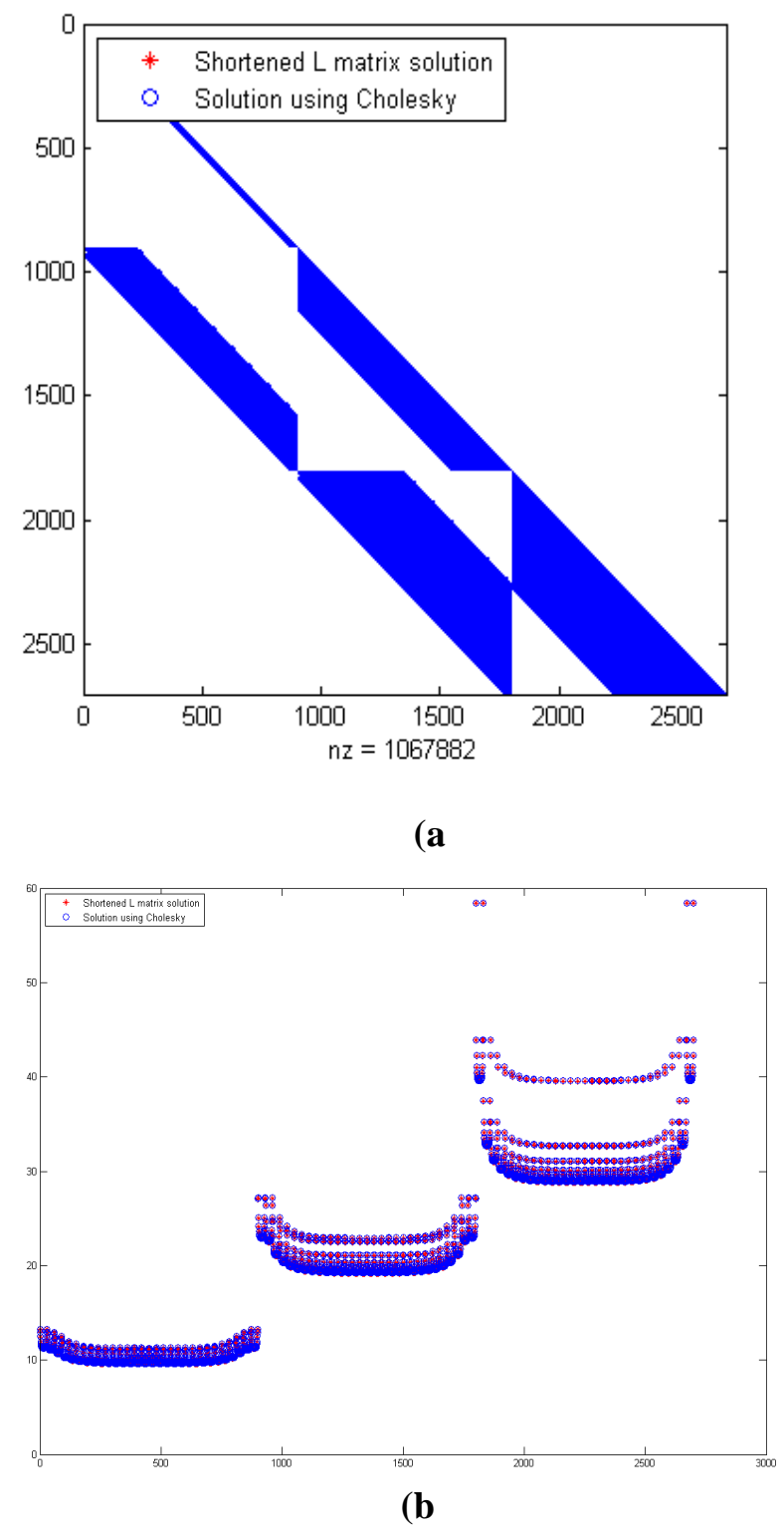

Figure 49 (a) Cholesky matrix computed using the intelligent Cholesky algorithm and (b) Comparison of temperatures computed using intelligent Cholesky and Matlab Cholesky algorithms at a threshold of 160. 


\subsubsection{Results with Criterion 3}

In this subsection, criterion 3 has been used for the computation of the lower triangulated Cholesky matrix with different tolerance levels between $10^{-8}$ and $10^{-3.5}$. In Figure 50, the ratio of maximum error at any DOF, number of FLOPS and total non-zero values appearing in the Cholesky matrix in comparison with Standard or Matlab Cholesky algorithms are plotted. It has been found that the number of FLOPS has been reduced to less than 5\% compared to the results obtained using the Standard Cholesky algorithm. The inaccuracy between the computed thermal nodal degrees of freedom using this algorithm at around 5\% FLOPS and the standard Cholesky algorithm is $\leq 0.1 \%$.

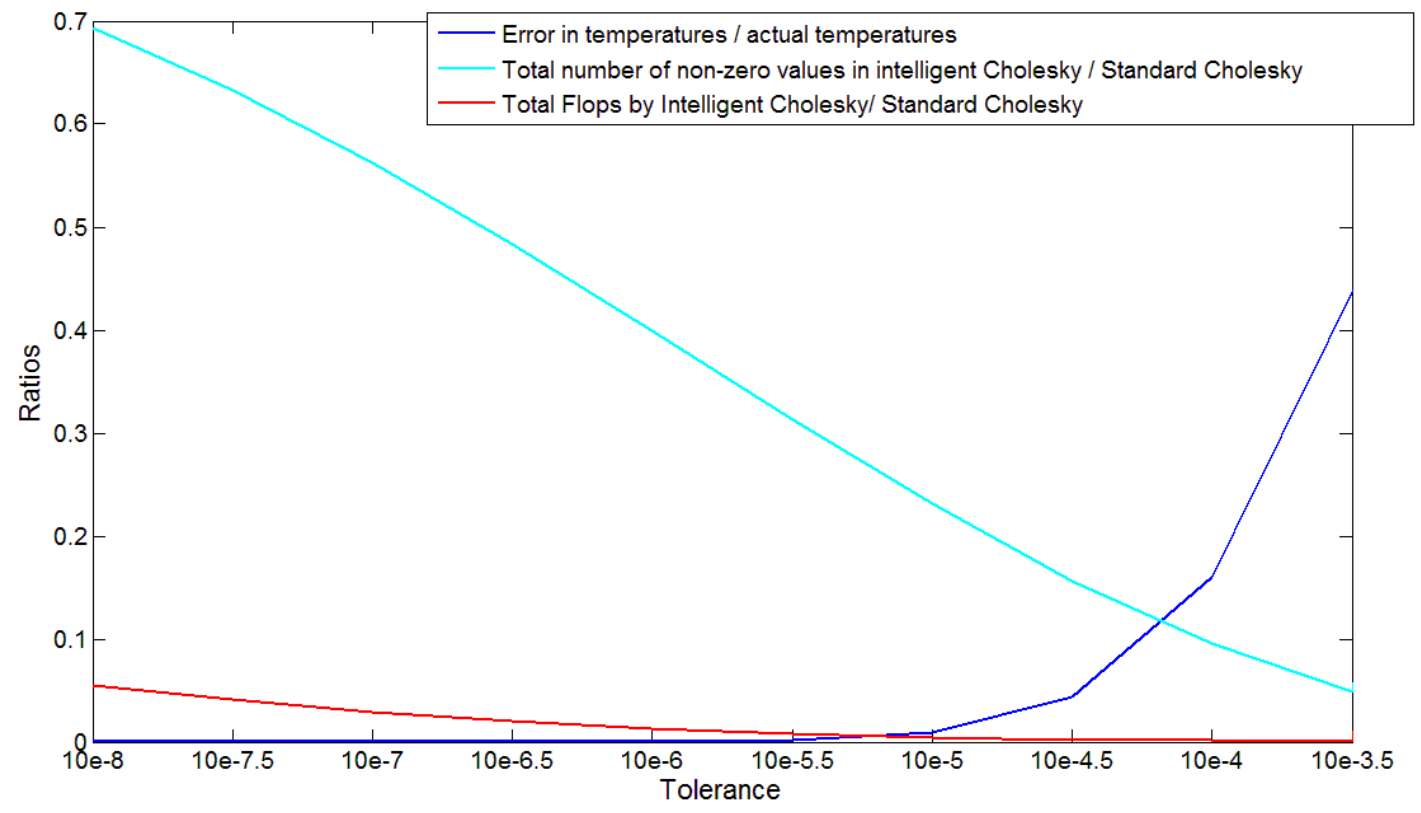

Figure 50: Figure (20). Comparison between standard Cholesky versus Intelligent Cholesky algorithm with criterion 3 . 


\subsection{Proposed Transform based Cholesky Decomposition}

A sample Fourier transform of a typical row of a Cholesky decomposition matrix has been shown in Figure 51. The Fourier transform of other rows is found to follow a strikingly similar pattern. In the proposed work, such a pattern could be used for matrix data storage along with fast and computationally efficient inner products of any two rows of the lower triangulated Cholesky matrix. This can be further extended to row and column operations involved in general matrix algebra computations. This will involve numerical experiments with various transforms such as wavelet decomposition (involves localizations in both frequency and time domains) along with traditional Fourier transforms (involves localizations explicitly in the frequency domain).

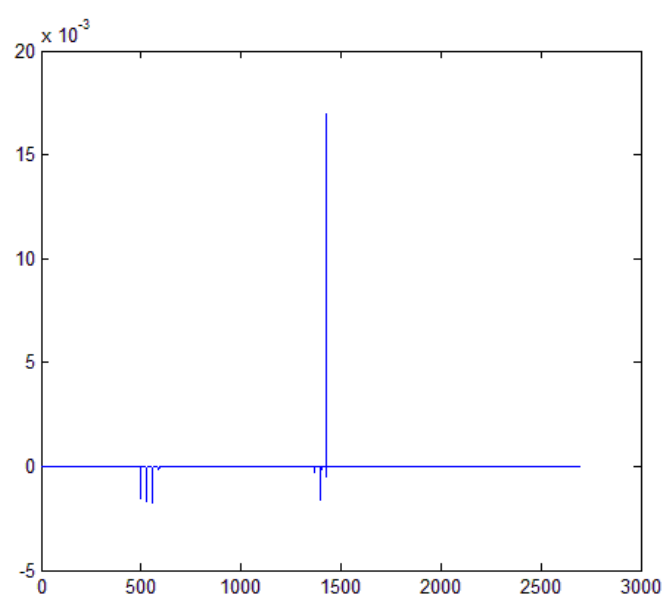

(a)

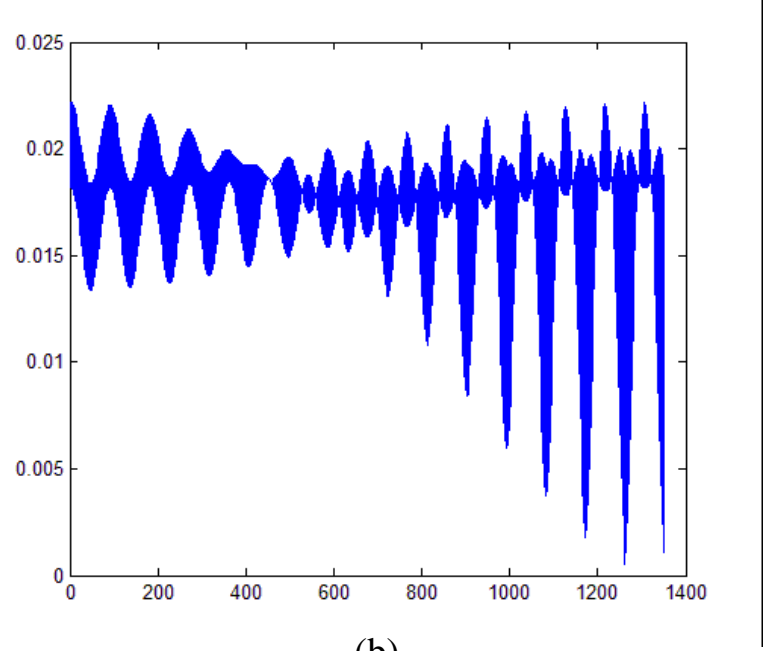

(b)

Figure 51 A typical row of the Cholesky decomposition matrix. (b) Fourier transform of the typical row of a Cholesky decomposition matrix 


\subsection{Conclusions}

A new modified Cholesky Decomposition algorithm has been developed. The computational cost to compute Cholesky decomposition is found to be reduced with the proposed Intelligent Cholesky Decomposition involving filtering of small values and numerical operations below a certain numerical threshold. Some observations about the Intelligent Cholesky decomposition are as follows:

- Boundary conditions have significant effects on the number of FLOPS which can be reduced using the proposed algorithms

- For dynamic problems, criterion 3 is ideal since it enables filtering of insignificant values arriving from matrix multiplications. The number of FLOPS can be reduced to less than $5 \%$ of the total FLOPS.

- For cases involving quasi-static problem descriptions in which iterative solution schemes are seldom implemented, a neighborhood based criterion such as criterion 2 is ideal. The number of FLOPS could be reduced to $20 \%$ of the total FLOPS required in standard Cholesky Decomposition. Improvement to this criterion is possible (such as criterion 5) by checking the distance in connectivity for both rows which will be involved in an inner product with each other.

- A Fourier or wavelet transform will be further used to improve the speed of computations as well as data storage efficiency for these matrices.

General applications of the new algorithm are as follows:

- Fast simulation of linear and nonlinear problems with sparse symmetric positive definite matrices. 
- Fast simulation of transient dynamic problems involving repeated inversion and multiplication of matrices.

- Efficient storage and retrieval of decomposition matrices or corresponding inverses.

- Efficient algorithms for Eigen decompositions of matrices such as $B^{-1} A$ where $B$ is a symmetric positive definite matrix and where $A$ does not share the positive definite property of its matrix structure with $B$. 


\section{CHAPTER 7}

AN ENERGY DISSIPATIVE CONSTITUTIVE MODEL FOR MULTI-SURFACE INTERFACES AT WELD

\section{DEFECT SITES IN ULTRASONIC CONSOLIDATION}

\subsection{Introduction}

Metal based additive manufacturing processes include both thermally-induced fusion processes and solid state processes. Some of the benefits of a solid state process lie in unique microstructures made possible because of near room temperature processing and the avoidance of solidification cooling induced residual stresses in the built part. These benefits can be further exploited if closed loop control of these processes becomes possible. The major hurdle in this is the computational complexity of the contact simulations, especially at the defect sites and the layer interfaces, involved in solid state processes like Ultrasonic Consolidation (UC). The present work is focused on development of a novel framework for interface simulations with the capability to include friction and various large deformation, nonlocal and geometrical nonlinearities involved in metal deformation.

\subsection{Ultrasonic Consolidation}

Ultrasonic consolidation (UC) is a solid-state additive manufacturing process which combines ultrasonic metal welding and milling to produce three dimensional objects. The 
process uses the high frequency ultrasonic shearing vibration at low amplitude and a normal force to break the oxide layers between two foils and then bond them by bringing together two nascent foil surfaces. Figure 52 shows a schematic of the UC process. The weld quality achieved in the process is sensitive to machine parameters. This sensitivity calls for better optimization of machine parameters through well informed computer simulations.

The UC process involves nonlinear plasticity which is inherently multi scale in space as well as time. The nonlinear and nonlocal plasticity in the process is dependent on the rate of deformation as well as size effects. The phenomenon of acoustic softening during UC is governed by these complicated material behaviors along with rate dependent dislocation dynamics. Continuum plasticity can be used to model the average phenomenon involved but it cannot account for the grain fragmentations or grain structure evolutions, which are at the core of the process if the quality of the weld is in question. A computational framework involving dislocation density based crystal plasticity $[133,134]$ has been developed. The computational cost involved in these simulations can be high and thus further work is in order to reduce it by an order of magnitude in order to make it fast enough to work with machines for online process control.

In the present work a novel framework for developing interfacial constitutive models is presented to evaluate the macroscopic response of interfaces for various surface roughness values and material properties along with homogenization of dislocation dynamics near interfacial regions. 


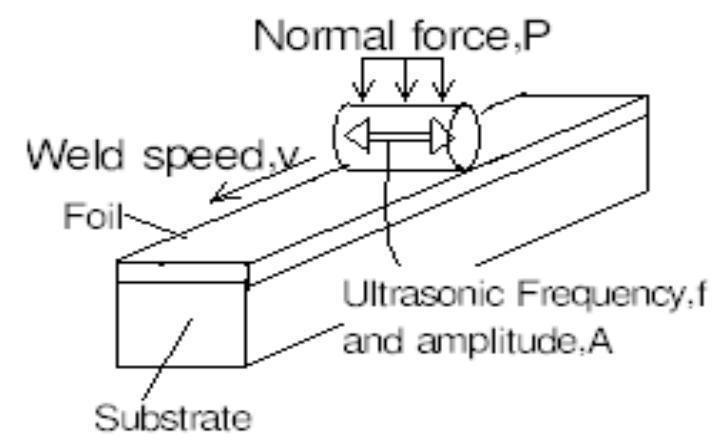

Figure 52 Schematic of UC process [133]

\subsection{Interfacial Constitutive Model}

The concept of an interfacial constitutive model has been researched for various applications like crack growth and propagation. The cohesive zone model $[135,136]$ is considered one of the pioneering interfacial constitutive models. The concept of a cohesive zone model can be further improved to simulate continuum level constitutive modeling of interfacial friction and sliding behavior [137]. Interfacial constitutive model literature is discussed in this subsection because it is closely related to the new formulation attempted in this work. The Extended Finite Element (XFEM) [138] methodology recently employed for multi-material and discontinuity simulations is another important development which has the capability to deal with various jump conditions involved at material interfaces. XFEM deals with discontinuities by decoupling a macroscopic average problem from the discontinuity problem at the FEM point integration level in order to calculate its effect on the stiffness matrices and macroscopic unbalanced forces. The decoupling of two problems rely on the functional evaluation of the coupling between the average macroscopic problem and the discontinuous behavior which could be a multi-scale phenomenon. 


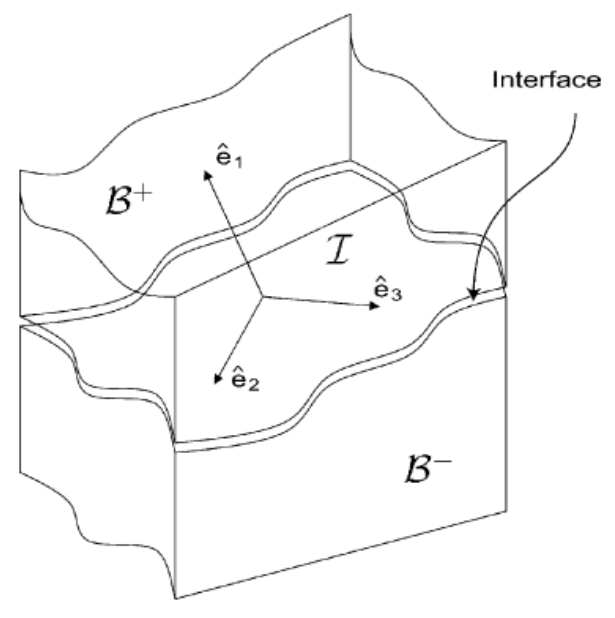

Figure 53 Interface between two bodies [137]

A cohesive interface between two bodies as shown in Figure 53 can be described as a contact interface between two bodies with or without any bonding mechanisms present at the interface. A constitutive model [137] for such an interface was developed by Su et. al and is discussed here to consider its potential application to Ultrasonic Consolidation. Consider two bodies $\beta^{+}$and $\beta^{-}$separated by an interface $\mathfrak{J}$. Assume $\left\{\hat{\boldsymbol{e}}_{1}, \hat{\boldsymbol{e}}_{2}, \hat{\boldsymbol{e}}_{3}\right\}$ is an orthonormal triad. The basis $\hat{e}_{1}$ is aligned with the normal $\boldsymbol{n}$ to the interface, and $\left\{\hat{\boldsymbol{e}}_{2}, \hat{\boldsymbol{e}}_{3}\right\}$ are in the tangent plane at any point $\overrightarrow{\boldsymbol{x}}$ in the plane.

Let $\delta$ denote the displacement jump across the cohesive surface, and $\boldsymbol{t}$ the power conjugate traction, such that $\boldsymbol{t} \dot{\boldsymbol{\delta}}$ gives the power per unit of the interface in the reference configuration. The displacement jump can be decomposed into plastic and elastic deformation as follows.

$$
\delta=\delta_{e}+\delta_{p}
$$

The applied power per unit area of the interface can be denoted by 


$$
t \dot{\delta}=t \dot{\delta}_{e}+t \dot{\delta}_{p}
$$

Let $\varphi$ denotes the free energy per unit surface area in the reference configuration. The dissipation per unit area can be written as follows.

$$
\Gamma=t \dot{\delta}_{e}+t \dot{\delta}_{p}-\dot{\varphi}
$$

Plastic deformation produces no stresses. This leads to

$$
t=K\left(\delta-\delta_{p}\right)
$$

The yield surface for the interface point can be described as the intersection of two convex surfaces corresponding to normal mechanism $\left(\emptyset_{1}\right)$ and shear mechanism $\left(\emptyset_{2}\right)$ respectively as shown in Figure 54.

$$
\begin{gathered}
\emptyset_{1}=t_{N}-s^{1} \leq 0 \\
\emptyset_{2}=\bar{\tau}+\mu t_{N}-s^{2} \leq 0
\end{gathered}
$$

The evolution of plastic deformation is described mathematically as

$$
\begin{gathered}
\dot{\delta}_{p}=\sum_{i=1}^{2} v^{i} \boldsymbol{m}^{i} \\
\boldsymbol{m}^{1}=\boldsymbol{n}
\end{gathered}
$$




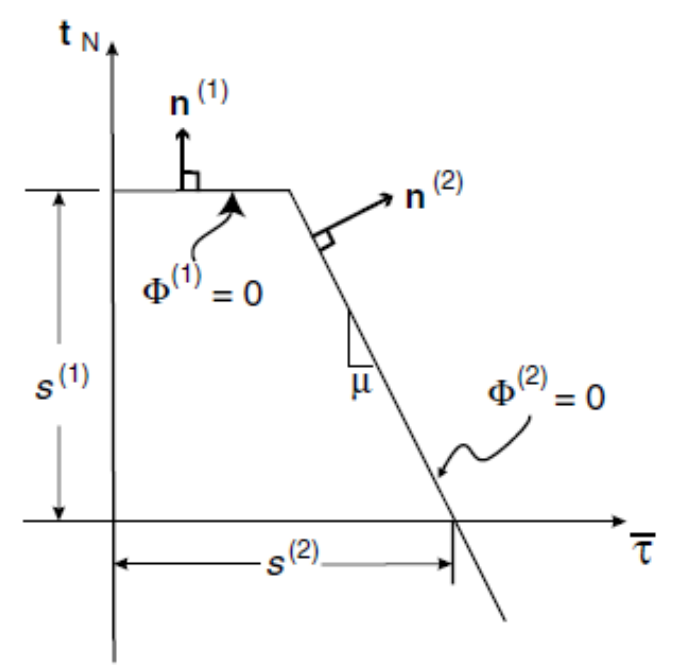

Figure 54 Schematic of the yield surface for normal and shear mechanisms [137].

$$
\boldsymbol{m}^{2}=\frac{\boldsymbol{t}_{\boldsymbol{T}}}{\bar{\tau}}
$$

This is a non-normal flow rule for the shear response. Finally the required consistency condition for plastic deformation is as follows.

$$
v^{i} \emptyset_{i}=0 \text {, when } \emptyset_{i}=0
$$

The consistency equation helps identify the possibility of plastic deformation. The magnitude of plastic deformation has to be further determined based on the evolution of $s^{i}$ as a function of time. The evolution mechanisms for $s^{i}$ are in general described as hardening and softening and their evolution is a function of dislocation dynamics; and a detailed crystal plasticity simulation is required in order to capture them for general loading and geometry scenarios with given macrostructure information on both sides of the interface. 


\subsection{Applications of the interfacial constitutive models}

This constitutive law will help in simulation of the process at the macroscopic scale, which will result in reduced computational complexity. Other applications for such a model include engineering problems such as cohesive modeling of crack interfaces during their propagation. The contact problem is also significant from the perspective of bonded or unbonded joints in metals. These energy dissipation laws will be also helpful in developing constitutive models and new interfacial elements for macroscopic vibration simulations.

The application of such an interfacial model in additive manufacturing other than the UC process will include support snapping simulation for Selective Laser Melting (SLM) where an initial crack in the lattice will grow and cause support structures failure. In case of SLM delamination the possibility of two successive layers built with significant recoat time can be also studied in depth. The friction stir surfacing process can be also modeled efficiently with the proposed simulation framework.

\subsection{Mathematical Formulation}

The schematic problem description is given in Figure 55. Simple 3D brick elements are used for the FEM simulation. 


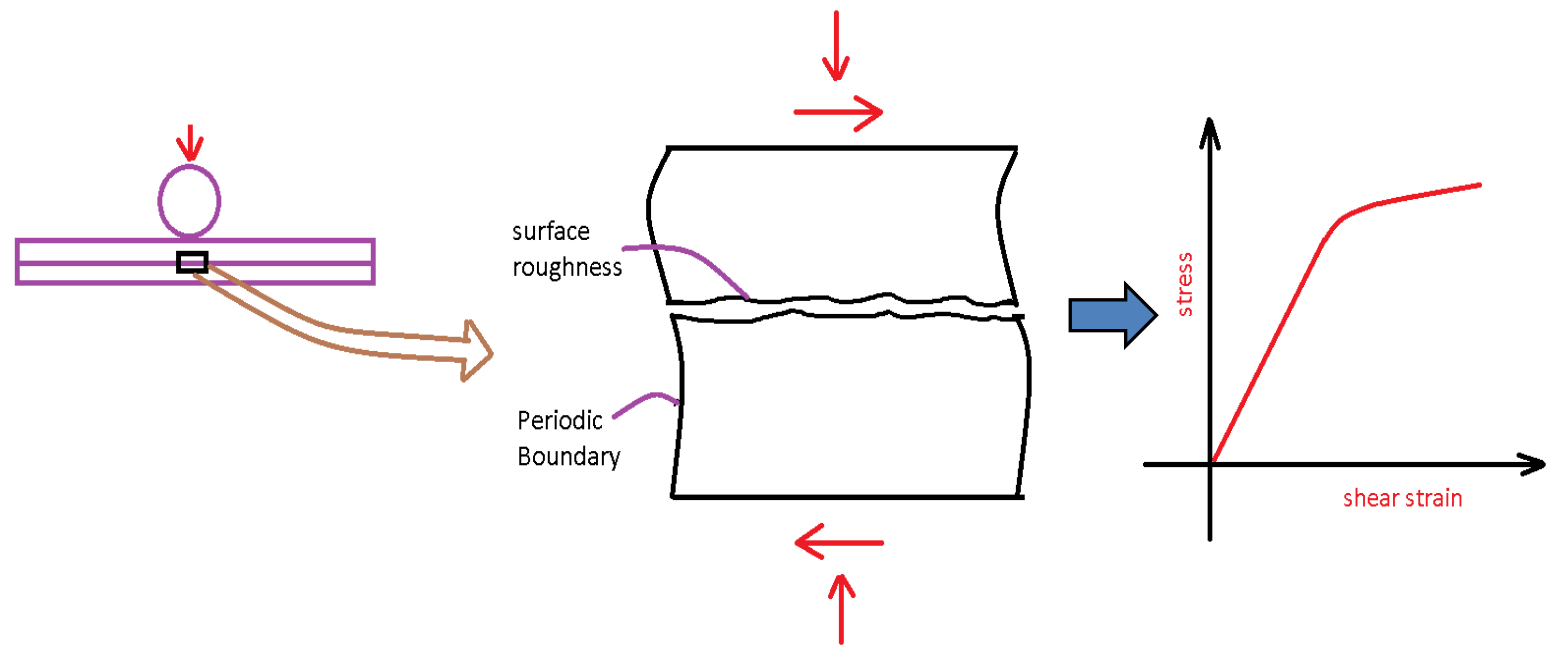

Figure 55: Schematic diagram presenting the concept of an interfacial constitutive model for a macroscopic interface contact

The virtual work equation is written in virtual work formulation as

$$
\begin{gathered}
\delta V=\int(\delta \varepsilon) C(\varepsilon) d v+\delta W(\delta u)=0 \\
\varepsilon=[B] \boldsymbol{q}
\end{gathered}
$$

The $\boldsymbol{q}$ vector is the nodal displacement vector. The equation above leads to the element stiffness equation which is

$$
K_{\text {elem }}=B^{T} C B
$$

The nodal equilibrium in the global coordinate system will lead to a final finite element equation which is

$$
\left[K_{\text {global }}\right] \boldsymbol{u}=\boldsymbol{F}_{\text {global }}
$$


The traditional approach to assemble a stiffness matrix from local to global coordinate systems is of individually substituting each value in the $K_{\text {elem }}$ into $K_{\text {global }}$. The present work includes development of a new mapping from local to global coordinate systems which will simplify the FEM assembly process in the case of contact problems where connectivity is updated as per the updated contact surface configuration.

$$
\left[K_{\text {global }}\right]=\operatorname{Perm}\left[K_{\text {Local }}\right] \operatorname{Perm}^{T}
$$

In case of changing contact surface configurations this can be modified as follows,

$$
\left[K_{\text {updated }}\right]=\operatorname{Perm}\left[K_{0}\right] \operatorname{Perm}^{T}
$$

where $\left[K_{0}\right]=($ Stiffness Matrix $)$ without any contact constraints, Perm $=($ Transformation matrix) which maps one configuration to another and has the following mathematical form, where $R$ is a mathematical relation or a second order tensor:

$$
\text { Perm }=A R B
$$

where $\mathrm{A}$ is a set of all nodes in the initial configuration and $\mathrm{B}$ a set of nodes in the final configuration.

The forces also follow a similar map as follows.

$$
\left[F_{\text {updated }}\right]=\operatorname{Perm}\left[F_{0}\right]
$$




\subsubsection{Contact Surface Constraints and solution of constrained simultaneous equations}

\subsection{Slave Nodes}

The contact surface constraints are based on the gap function to evaluate contact which is defined as

$$
\boldsymbol{g}(x, y)=u_{t 3}(x, y)-u_{b 3}(x, y)
$$

where $u_{t 3}(x, y)$ is the $\mathrm{z}$-direction displacement of the top surface at point $(\mathrm{x}, \mathrm{y})$. Similarly $b$ in $u_{t 3}(x, y)$ stands for the bottom surface. If the $\boldsymbol{g}(x, y)=0$ and shear is less than the friction resistance, then the top surface node is treated as a slave to the bottom surface. This is achieved by providing a constraint based on the shape functions of the bottom surface element as follows:

$$
q_{t}=N_{1} q_{1}+N_{2} q_{2}+N_{3} q_{3}+N_{4} q_{4}
$$

where $q_{t}$ is any $\mathrm{x}, \mathrm{y}$ or $\mathrm{z}$ displacement of a node on the top surface and $q_{i}$ are corresponding displacements on the bottom target element.

The constraint equations are solved by splitting the slave node stiffness matrix row into different rows. Each split row will be added to the master Degree of Freedom (DOF) row based on the $N_{i}$ in the constraint equation for the master DOF. This methodology is explained in Figure 56. 

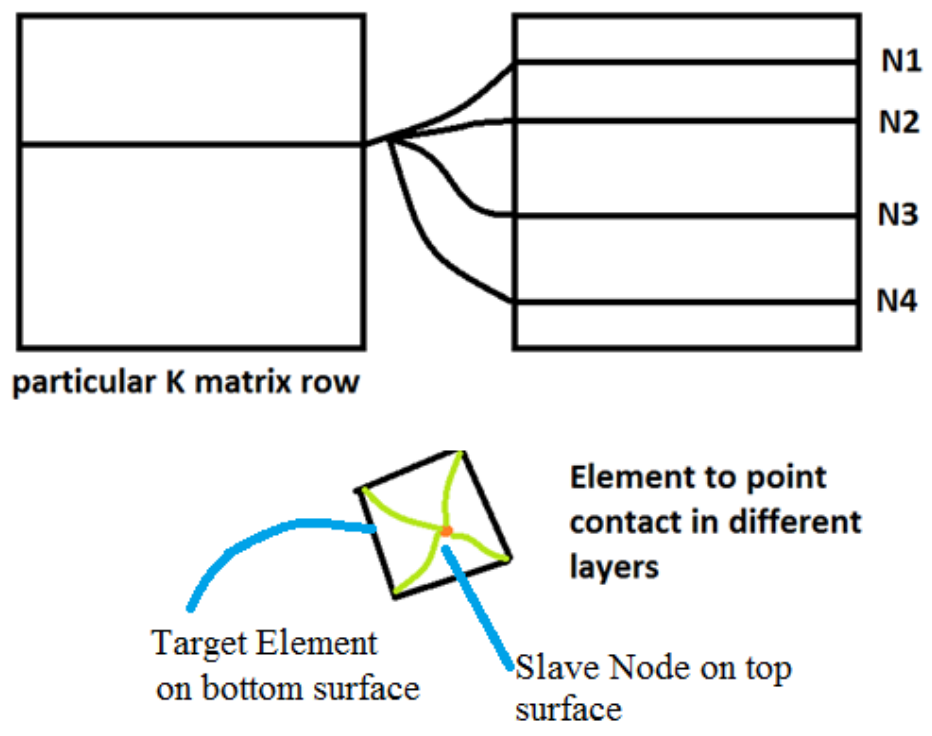

Figure 56 Schematic diagram representing a slave node constraint on a contact surface and its solution methodology by splitting slave node stiffness as per the constraint equation.

\subsection{Free Nodes}

A node is set free if it is sliding or has lost contact with the bottom surface.

\section{$3.0(\boldsymbol{g}(x, y) \geq 0)$ condition}

To simplify the algorithm, nodes that are impinging are first allowed to impinge and then are forced back to the other surface iteratively.

\subsection{Contact Simulation Algorithm}

The contact simulation algorithm is based on minimizing unbalanced force iteratively along with updated contact constraints. First contact constraints are updated which will induce some unbalance forces due to shear slip and change in stiffness. These unbalanced forces are solved in the new configuration which can further change the gap function and 
can change the contact constraints. The algorithm is presented in a flow chart format in Figure 57.

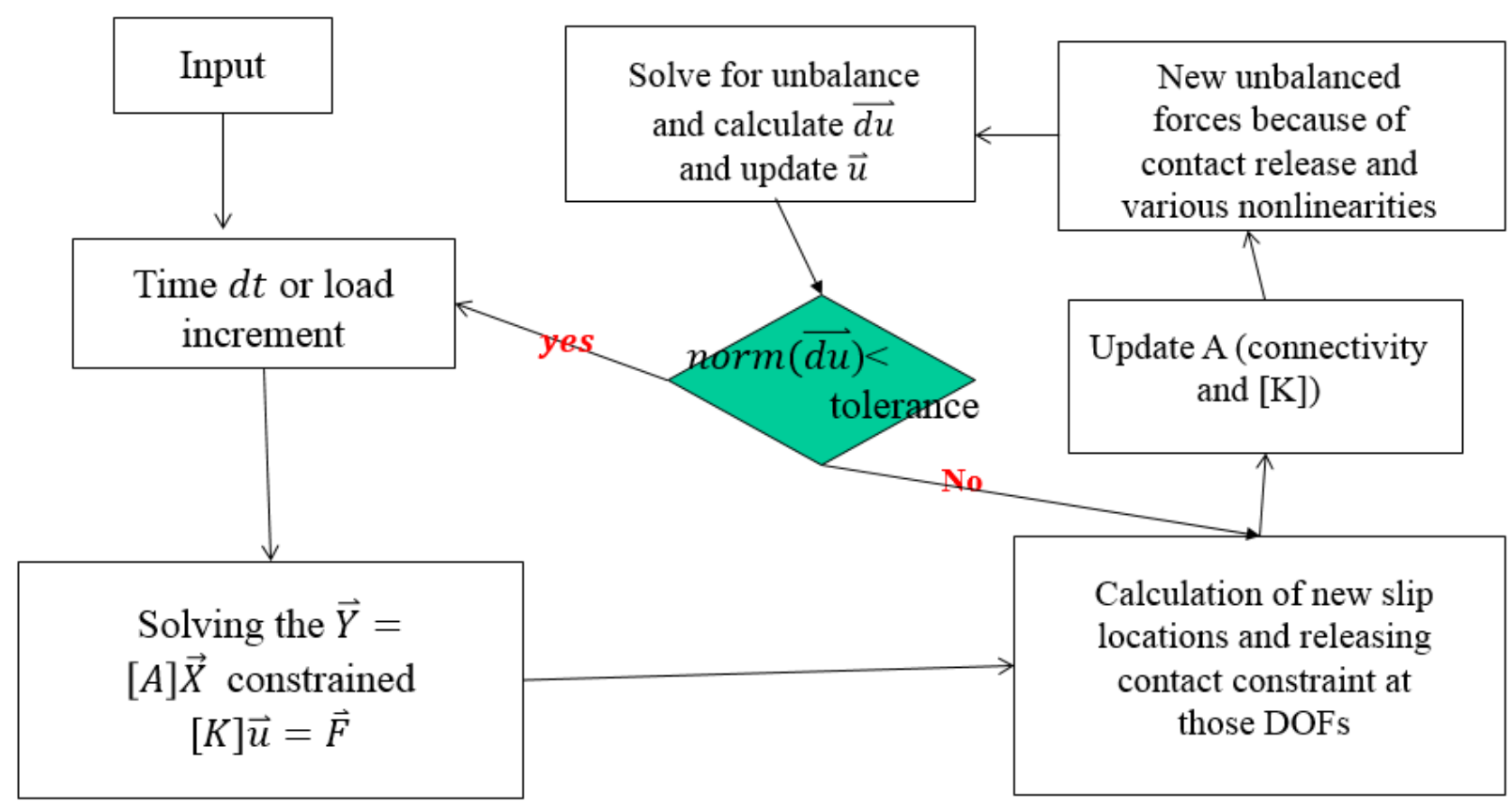

Figure 57 Flow chart showing FEM algorithm used for contact simulation

\subsection{Case Study}

A simple case of surface to surface contact is considered here. Two prismatic blocks of size $0.001 \times 0.001 \times 0.0005 \mathrm{~m}^{3}$ are in contact with each other as shown in Figure 58 . The z-direction height of each block is $0.0005 \mathrm{~m}$. The boundary conditions include a bottom surface fixed in all degrees of freedom and a top surface with slip and normal compressive displacement boundary conditions as shown in Figure 58. The details of the boundary conditions and material properties are as follows.

\section{Top Surface:}

$u_{1}(x, y)=\operatorname{slip}(t)$ 
$u_{2}(x, y)=0$

$u_{3}(x, y)=-0.00001 m$

\section{Bottom Surface:}

$u_{1}(x, y)=0$

$u_{2}(x, y)=0$

$u_{3}(x, y)=0$

Sides: No constraint

Material Properties: Linear elastic

$$
\begin{aligned}
& E=2 \times 10^{11} \mathrm{~N} / \mathrm{m}^{2} \\
& \mu=\text { poisons ratio }=0.3
\end{aligned}
$$

Friction coefficient $=0.3$
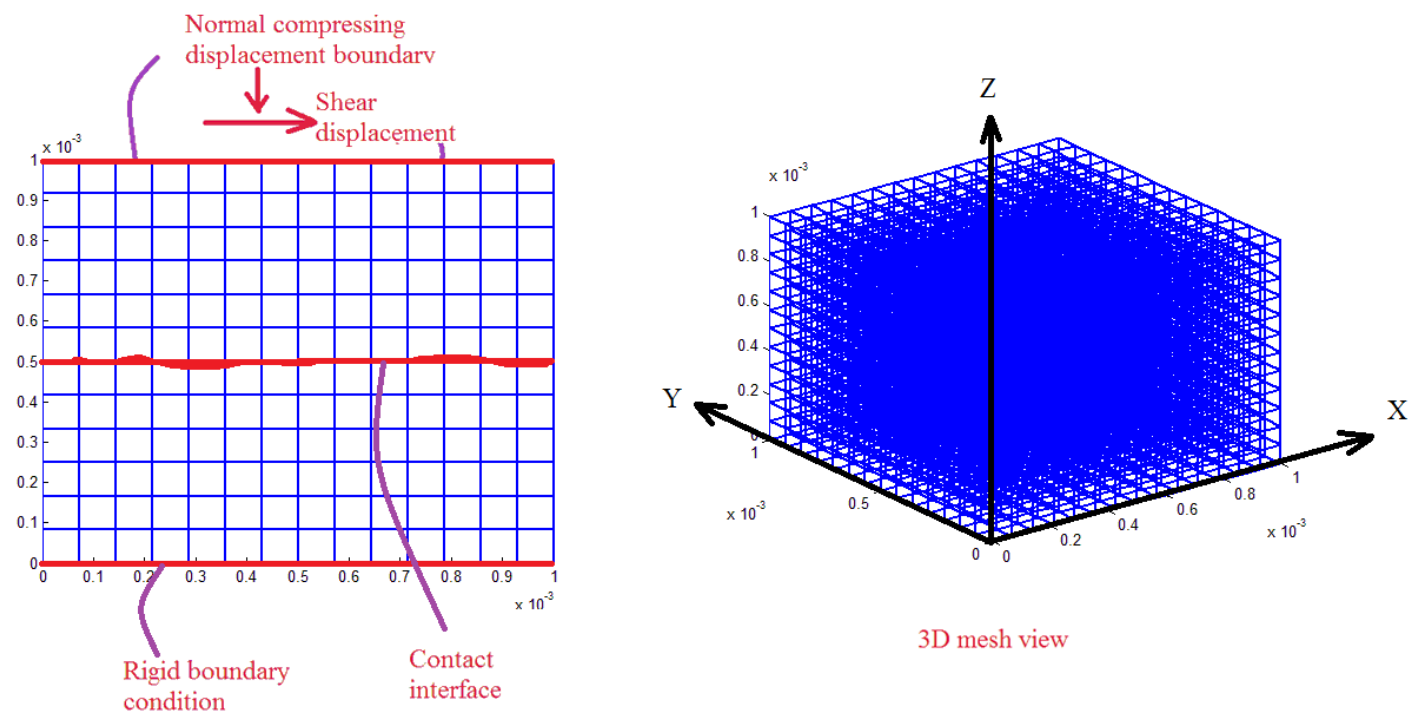

3D mesh view

Figure 58 Problem geometry and boundary conditions 


\subsection{Results and Discussion}

The present work is a small case study to verify whether future work deriving interfacial constitutive models is merited. The correct boundary conditions for extracting constitutive models are outside the focus of this work, as this work focused on formulating a FEM framework which can consider different boundary conditions and give high fidelity contact simulations. The parameters of interest in this case study are the macroscopic aggregate shear resistance and macroscopic strain.

The shear resistance of the contact surface is defined below. Considering the prismatic geometry of the model the total shear resistance for any cross-section will come out to be the same.

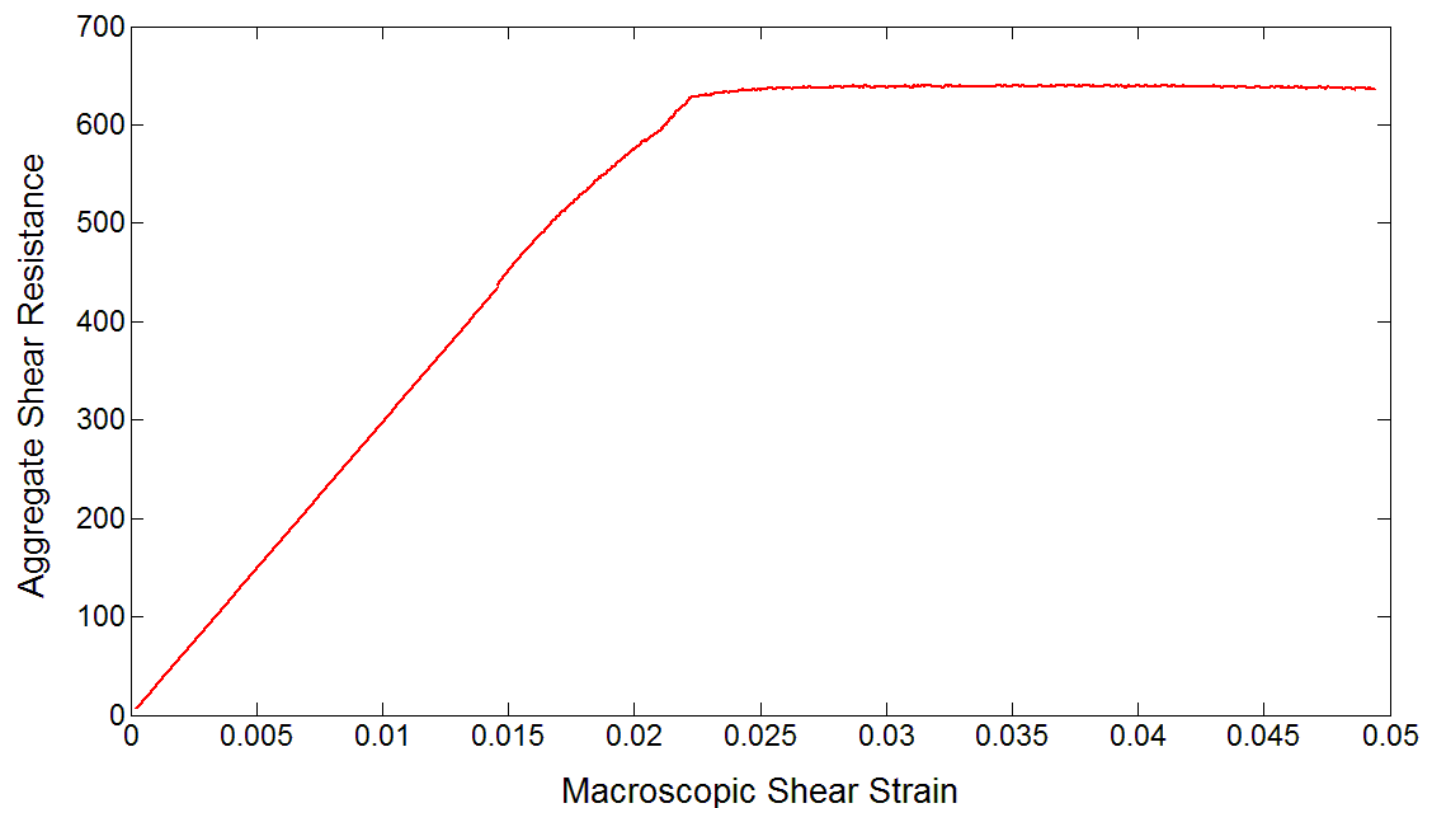

Figure 59: Macroscopic strain versus shear resistance plot 


$$
\begin{gathered}
\text { Aggregate Shear Force }=S=\int^{c / s \text { area }}\left(\sigma_{13}\right) d s \\
\text { Aggregate Normal Force }=N=\int^{c / s \text { area }}\left(\sigma_{33}\right) d s
\end{gathered}
$$

The plot of S versus slip given at the top surface is shown in Figure 59. The reason behind the fall of the shear resistance with increase in slip is due to contact being lost at the extreme left or right points due to the overhanging nodes at the end. Figure 60 shows a plot of macroscopic friction coefficient calculated as a ratio of $\mathrm{S}$ and $\mathrm{N}$ at each time instant during slip loading in the simulation. The reason for a macroscopic friction coefficient less than 0.3 of that of the actual interface can be attributed to the fact that the shear resistance at each point in the cross-section is not utilized completely in one particular configuration or point in time. Another way to explain it is that the 0.3 is the maximum upper bound that can be achieved.

The shear resistance will be totally lost at the overhangs because there is no material available on either side for contact friction as seen in the deformed shape shown in Figure 61. 


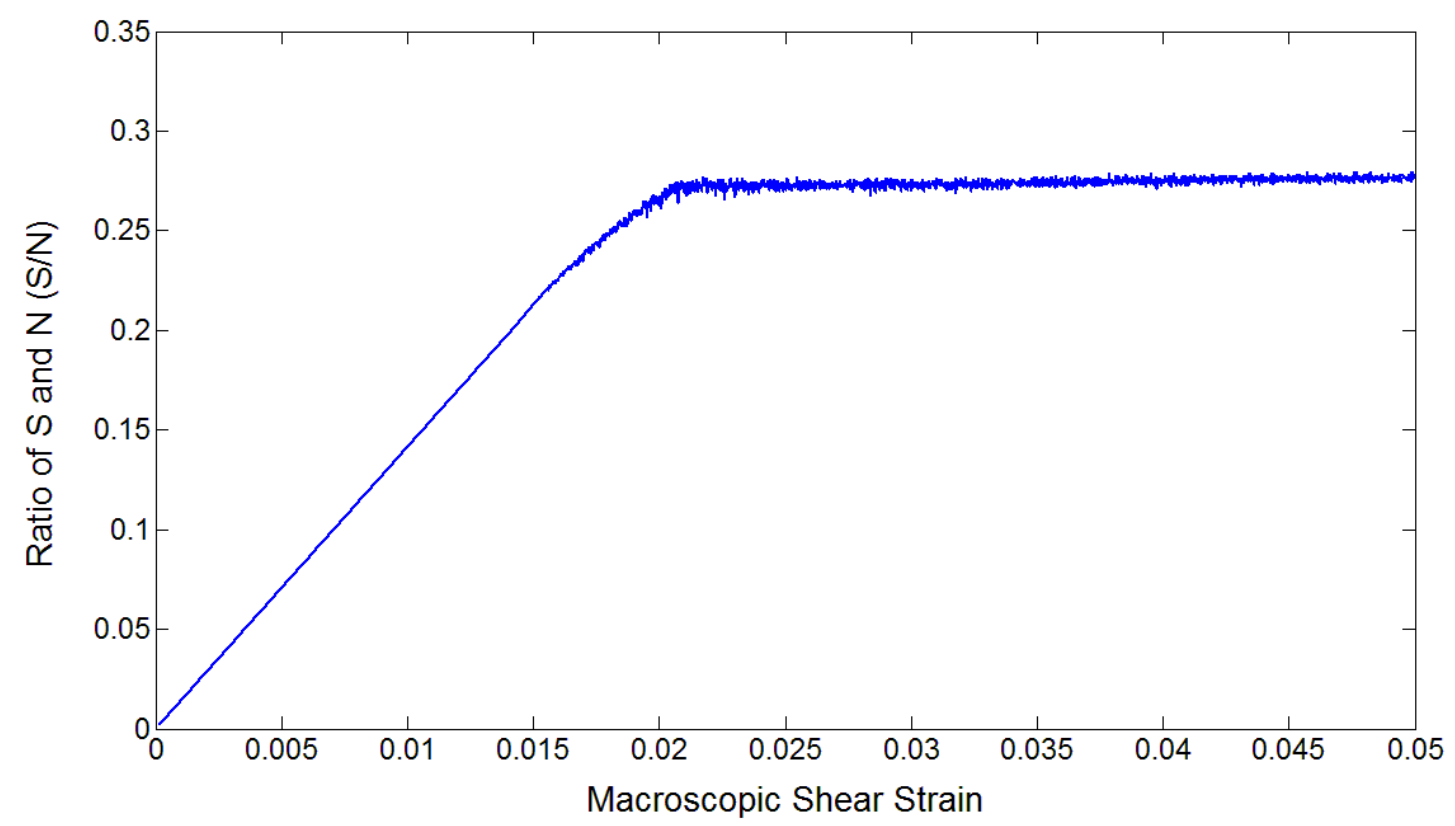

Figure 60: Plot of the macroscopic friction coefficient

\subsection{Conclusions and future work}

A novel framework for an interfacial friction model has been developed in the present work. The developed contact simulation framework shows good behavior at the contact interface. The novel features of this framework include:

- Mathematical framework for efficient assembly of FEM matrices

- Solution of constraint equations exactly without any additional computational cost

- No addition of penalty springs which can introduce spurious stiffening behavior at interface

The future work in this problem will include addition of the following features to the present framework in order to develop interfacial constitutive models or laws: 
- Nonlinear simulations with crystal plasticity based material constitutive models for metals.

- Inclusion of tangent stiffness at the current configuration (Geometric nonlinearity)

- Periodic boundary condition considering the case of deriving a constitutive model for a point on an interfacial surface.

- Inclusion of surface roughness

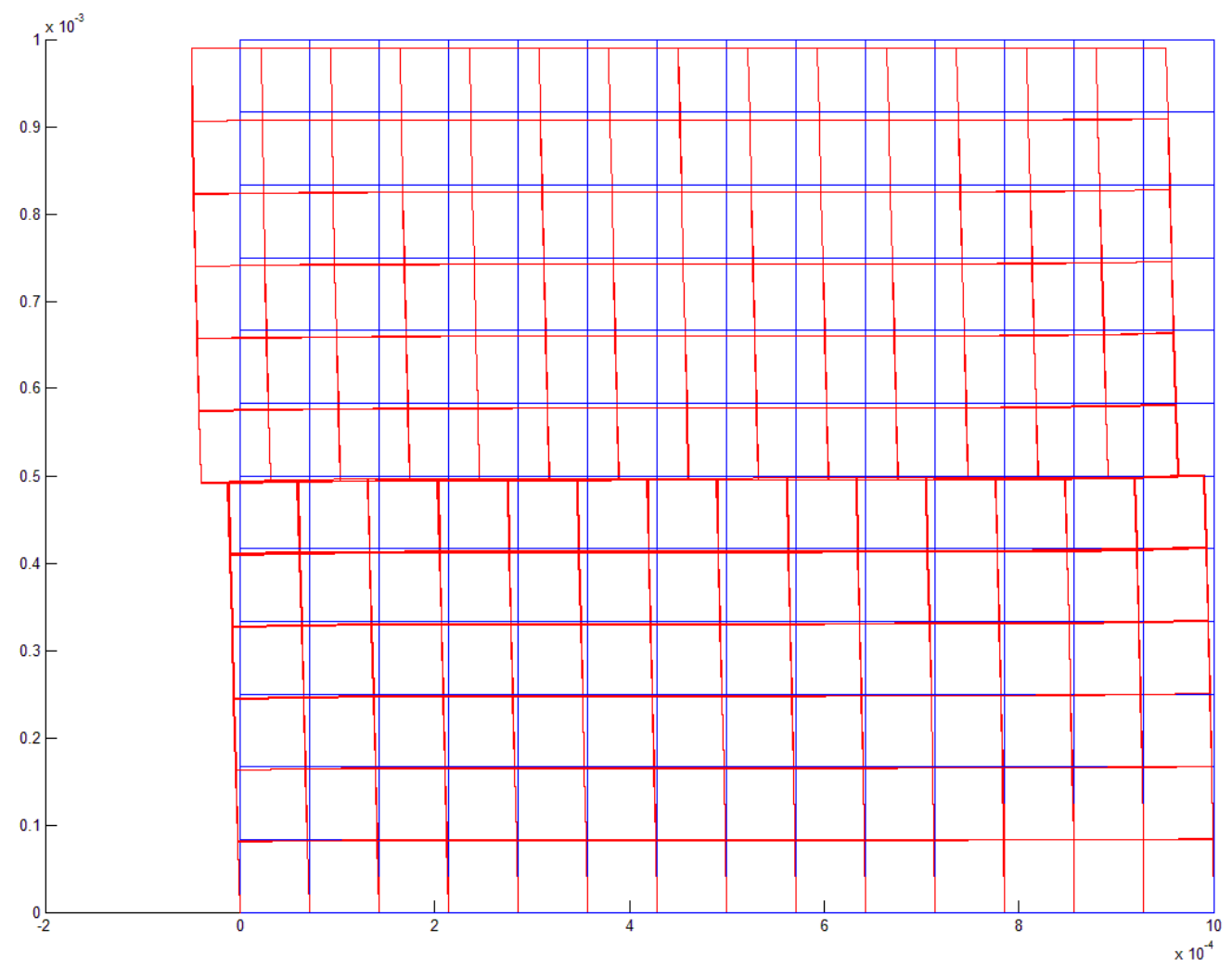

Figure 61: Deformed and un-deformed mesh at a macroscopic shear strain of 0.05 


\section{CHAPTER 8}

\section{A NOVEL CRYSTAL PLASTICITY AND SURFACE TO SURFACE CONTACT SIMULATION FRAMEWORK FOR SIMULATION OF ADDITIVE MANUFACTURING \\ PROCESSES}

\subsection{Introduction}

Surface to surface contact simulations are significant from the perspective of tribology and for solid state metal bonding processes such as those based on additive manufacturing. A detailed literature discussion of contact analysis and a novel energy dissipative constitutive model has been previously introduced [139]. In this paper, application of this contact model in conjunction with a detailed material deformation model for metal plasticity is introduced.

Metal plasticity can be simulated as continuum plasticity with yield functions. One of the well-known yield functions is a von-Mises surface. The disadvantage of using continuum plasticity has been in simulating the rate and texture dependence of the deformation. The yield functions have a scalar dimension and lack information of slip system orientation with respect to external loading along with detailed computation of 
lattice rotation as a function of grain orientation. The limitations of yield function based continuum plasticity can be overcome with Crystal Plasticity based constitutive modeling along with finite element modeling. The following sections include discussion of nonlinear finite element algorithms that include crystal plasticity constitutive model formulations introduced in the present work.

\subsection{Plastic deformation and FEM implementation}

The plastic deformation gradient can be decomposed into the following form:

$$
\mathrm{F}=\mathrm{F}_{\mathrm{e}} \mathrm{F}_{\mathrm{p}}
$$

where $F_{e}$ is elastic deformation and $F_{p}$ the plastic deformation gradient. This elastic plastic decomposition implies that plastic deformation occurs before the elastic deformation can occur. The elastic 2 nd Piola-Kirchhoff stress tensor induced by $F$ is:

$$
P K_{2}=C\left(F_{e}^{T} F_{e}-I\right)
$$

where $C$ is the stress strain relation between elastic stress and strain for a point inside the lattice. The above equation also has implications such that the plastic deformation gradient does not induce any stress. The $P K_{2}$ stress has its traction directions and area in the original reference configuration. This can be corrected by converting $P K_{2}$ into Cauchy stress as follows:

$$
\sigma=\frac{1}{\operatorname{det}\left(F_{e}\right)}\left(F_{e} \sigma F_{e}{ }^{T}\right)
$$

The Cauchy stress when resolved on each slip system of the crystal governs plastic deformation rate $(L p)$. The stress resolution has been performed in order as follows. 
1. Stress resolves along the grain lattice orientation using Euler angle information available as shown in Figure 62.

2. Stress resolved in the lattice orientation has been further resolved in the slip plane normal direction to determine traction vector on the slip plane.

3. The traction has been resolved in normal and in the slip direction to determine normal stress on slip plane and the resolved shear stress (RSS) for the slip direction.
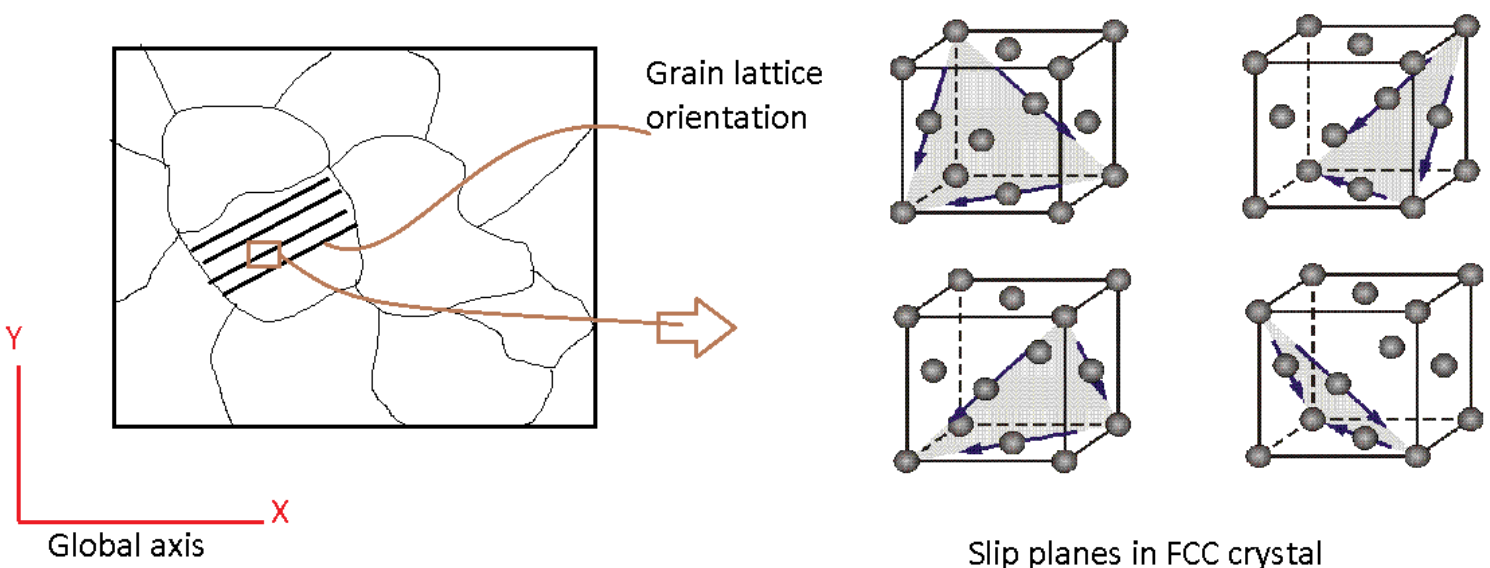

Slip planes in FCC crystal

Figure 62 Schematic representing (a) microstructure global axis and (b) the grain axis [140].

The plastic deformation rate is determined using the following relationship:

$$
L p=\dot{F}_{p} F_{p}^{-1}=\sum_{\alpha=1}^{N} \dot{\gamma}^{\alpha}\left(m_{\alpha} \otimes n_{\alpha}\right)
$$

where

$m_{\alpha}:$ slip orientation

$n_{\alpha}:$ slip plane normal 
$N$ : Total number of slip directions

$\dot{\gamma}:$ Slip velocity

The slip velocity is governed by a flow rule as follows:

$$
\dot{\gamma}^{\alpha}=\dot{\gamma}_{0} \operatorname{sign}\left(\tau^{\alpha}\right)\left(\frac{\tau^{\alpha}}{g^{\alpha}}\right)^{m}
$$

where $\gamma_{0}$ and $\mathrm{m}$ are slip system material parameters. $g^{\alpha}$ has been defined as the Critical Resolved Shear Strength (CRSS) of the slip plane. The $\dot{\gamma}^{\alpha}$ determines $L p$ which has been used to determine evolution of $F_{p}$ and the hardening or softening of the point as a function of evolution of stress at the same point. These evolutions have been used to calculate the stress strain relation tensor as derived in the following section.

\subsection{Stress Jacobian Derivation}

The stress Jacobian Derivation is conceptually similar to other work in the literature [142]. The approach used in the present work is based on tensorial derivatives, in contrast to variational approaches used in the literature. A point is subjected to a deformation gradient increment from $F(t)$ to $F(t+d t)$. The deformation increment is first applied elastically, resulting in complete elastic loading, followed by plastic relaxation as shown in Figure 63. 


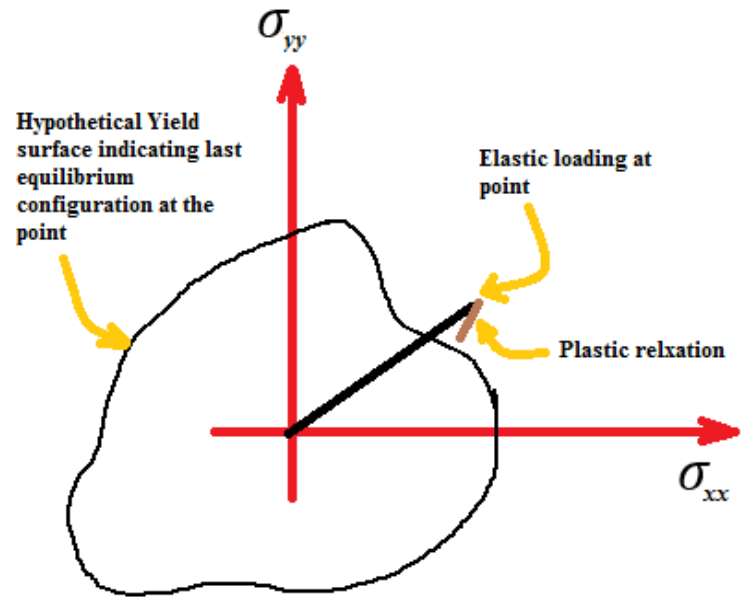

Figure 63 Schematic diagram showing elastic loading followed by plastic unloading The loading and unloading is represented by a single equation:

$$
\sigma(\tau)=\frac{1}{\operatorname{det}\left(F_{e}\right)}\left(F_{e} \sigma_{t r} F_{e}^{T}\right)
$$

where

$$
\begin{gathered}
\sigma_{t r}=\frac{1}{2} C\left(F_{e}{ }^{T} F_{e}-I\right)-\sum_{\alpha=1}^{N} C^{\alpha} \gamma d t \\
C^{\alpha}=\frac{1}{2} C\left(A S^{\alpha}+S^{\alpha T} A\right) \\
A=F_{e}{ }^{T} F_{e}
\end{gathered}
$$

The Jacobian has been derived using tensorial derivatives as follows.

$$
J=\frac{\partial \sigma}{\partial \varepsilon}=\frac{\partial \sigma}{\partial F} \frac{\partial F}{\partial \varepsilon}=\frac{\partial \sigma}{\partial F}\left(\frac{\partial \varepsilon}{\partial F}\right)^{-1}
$$

The first term in the above chain of derivatives is calculated as follows. 
Table 4 Plastic deformation finite element algorithm with crystal plasticity based constitutive formulation

For step $=1$ to number of time steps

Apply external boundary increment:

$$
\delta F=\text { Increment in external boundary loading }
$$

Forload_unload_cycle $=1$ to till convergence

a) overall geometry Elastic Loading

- Retrieve Jacobian information off all integration points in all elements.

- Calculate stiffness matrix $[\mathrm{K}]$ with BBAR integration procedure inside element.

- $\quad$ Solve $[K] \delta u=\delta F$

- $u=u+\delta u$

b) Plastic relaxation

- Retrieve displacement vector for each element

- Determine $\boldsymbol{F}$ at each integration point

- Calculate Jacobian at integration points with increments in each $F_{k l}$ given $C_{i j k l}$.

- Calculate stress at each integration point using finite strain definition.

- Calculate internal force in each element using $F_{\text {internal }}=\int_{v} B^{T} \sigma d v$

- Assemble internal forces and determine unbalanced force vector and save it as $\delta F$.

- $\quad$ Determine the $L p$ and update $F_{p}=(I+d t(L p)) F p$

end

end 


$$
P \delta F=\frac{\partial \sigma}{\partial F} \delta F
$$

Here the $\delta F$ is the direction or perturbation required in tensorial derivatives.

$$
\begin{gathered}
\frac{\partial \sigma}{\partial F} \delta F=-\operatorname{det}\left(F_{e}\right)^{-1} F_{e}^{-1}(\delta F) F_{e}\left(\sigma_{t r}\right) F_{e}^{T}+\operatorname{det}\left(F_{e}\right)^{-1}(\delta F) F_{p}^{-1} \\
\left(\sigma_{t r}\right) F_{e}^{T}+\operatorname{det}\left(F_{e}\right)^{-1} F_{e}\left(\frac{\partial \sigma_{t r}}{\partial F} \delta F\right) F_{e}{ }^{T}+\operatorname{det}\left(F_{e}\right)^{-1} F_{e}\left(\sigma_{t r}\right) F_{p}^{-T}(\delta F) \\
\frac{\partial \sigma_{t r}}{\partial F} \operatorname{del} F=F_{p}^{-1}(\delta F) F_{e}+F_{e}{ }^{T} F_{p}^{-1}(\delta F)-\sum_{\alpha=1}^{N} C^{\alpha} \frac{\partial \dot{\gamma} d t}{\partial \sigma} \frac{\partial \sigma}{\partial F} \delta F-\sum_{\alpha=1}^{N} \dot{\gamma} d t \frac{\partial C^{\alpha}}{\partial F}
\end{gathered}
$$

The $\frac{\partial \sigma}{\partial F} \delta F$ calculated in the above equations is a function of the $\delta F$ perturbation tensor. In order to overcome this difficulty $d e l F$ is incremented at each $F_{i j}$ separately and then combined together to determine the complete $\frac{\partial \sigma}{\partial F}$ as a fourth order tensor.

\subsection{Nonlinear Finite Element Algorithm}

The nonlinear finite element algorithm is based on the concept of global loading using the Jacobian at each point and global stiffness matrix. This step is followed by unloading at each point. This algorithm is outlined in Table 4.

\subsection{Case Study}

The formulated viscoplastic constitutive model has been used to simulate a uniaxial tensile test. The objective of this study is to demonstrate conceptual accuracy of the mechanics and physics involved in the present constitutive model formulation along with a nonlinear plasticity algorithm. Single crystal copper material properties [143] used in the simulation are as follows. 


\begin{tabular}{|c|c|c|c|c|c|c|}
\hline$\tilde{y}_{0}$ & $\mathrm{~m}$ & $g_{0}$ & $g_{s}$ & $h_{0}$ & $h_{s}$ & $q$ \\
\hline $0.001 s^{-1}$ & 15 & $16 \mathrm{MPa}$ & $70.4 \mathrm{MPa}$ & $132 \mathrm{MPa}$ & $8 \mathrm{MPa}$ & 1.4 \\
\hline
\end{tabular}

This simulation framework has been further combined with the interfacial contact simulation algorithm previously mentioned [139]. Contact simulations have been conducted with problem description same as that of given in Section 7.7. Dimensions of the individual block have been assumed to be $0.1 \mathrm{~m} \times 0.1 \mathrm{~m} \times 0.05 \mathrm{~m}$.

\subsection{Results and Discussions}

Nonlinear plastic deformation simulations often have trouble finding convergence and stability. It has been observed that the algorithm presented here is quite stable and has desired convergence characteristics.

The uniaxial tensile testing simulation has been conducted without hardening in order to check solution stability and convergence. The average stress vs macroscopic strain is shown in Figure 64. The absence of a hardening mechanism has resulted in plastic yield followed by linear variation between average stress and applied macroscopic strain. This phenomenon has been attributed to evolution of the Stress Jacobian. The variation of the $C_{33}$ component of the Stress Jacobian with applied macroscopic strain is presented in Figure 65 . The initial value of $2.22 \times 10^{5}$ represents the elastic region followed by yielding which results in a new stabilized value at $2.135 \times 10^{5}$. The plastic deformation increment for each time step becomes steady as shown in Figure 66. Similar sets of plots are shown for a case of axial displacement controlled loading followed by relaxation in Figure 67, 
Figure 68 and Figure 69. The simulation here has shown characteristics of viscoelastic behavior with a crystal plasticity based constitutive model.

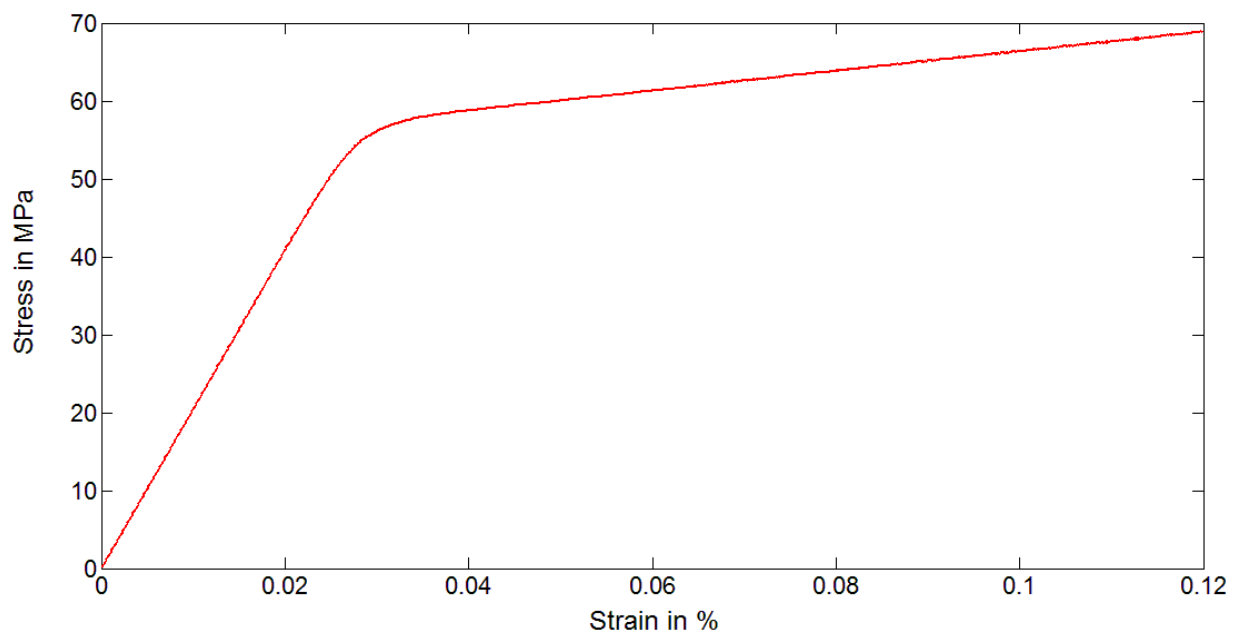

Figure 64 Plot of stress vs strain for the case of monotonic uniaxial tensile loading

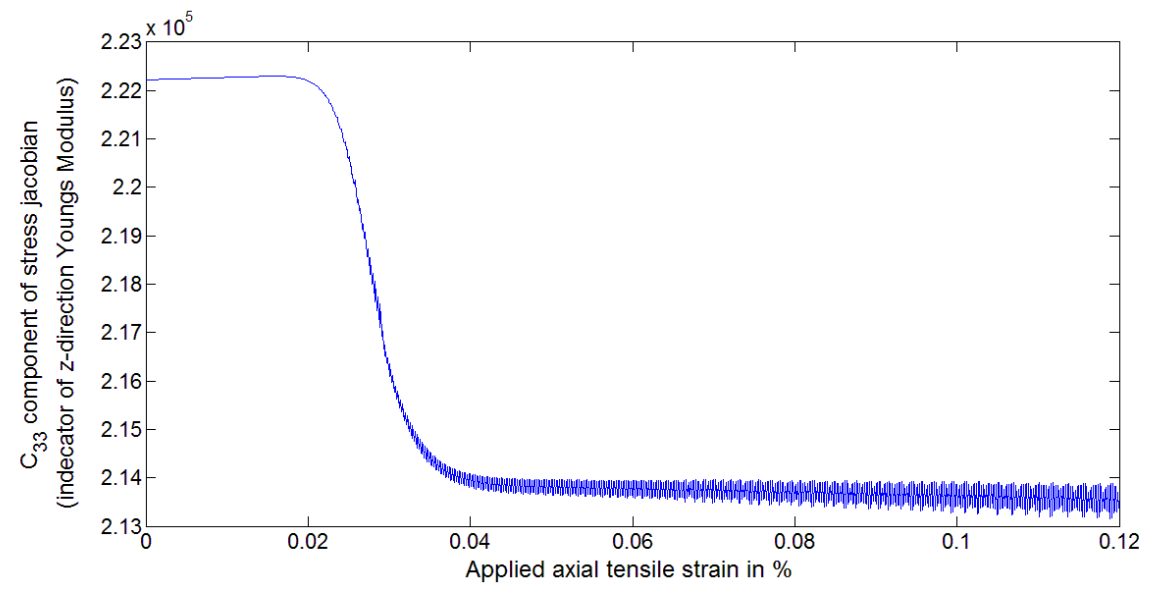

Figure 65 plot of $C_{33}$ (indicator of elastic modulus in the z-direction) vs time for the case of monotonic uniaxial tensile loading 


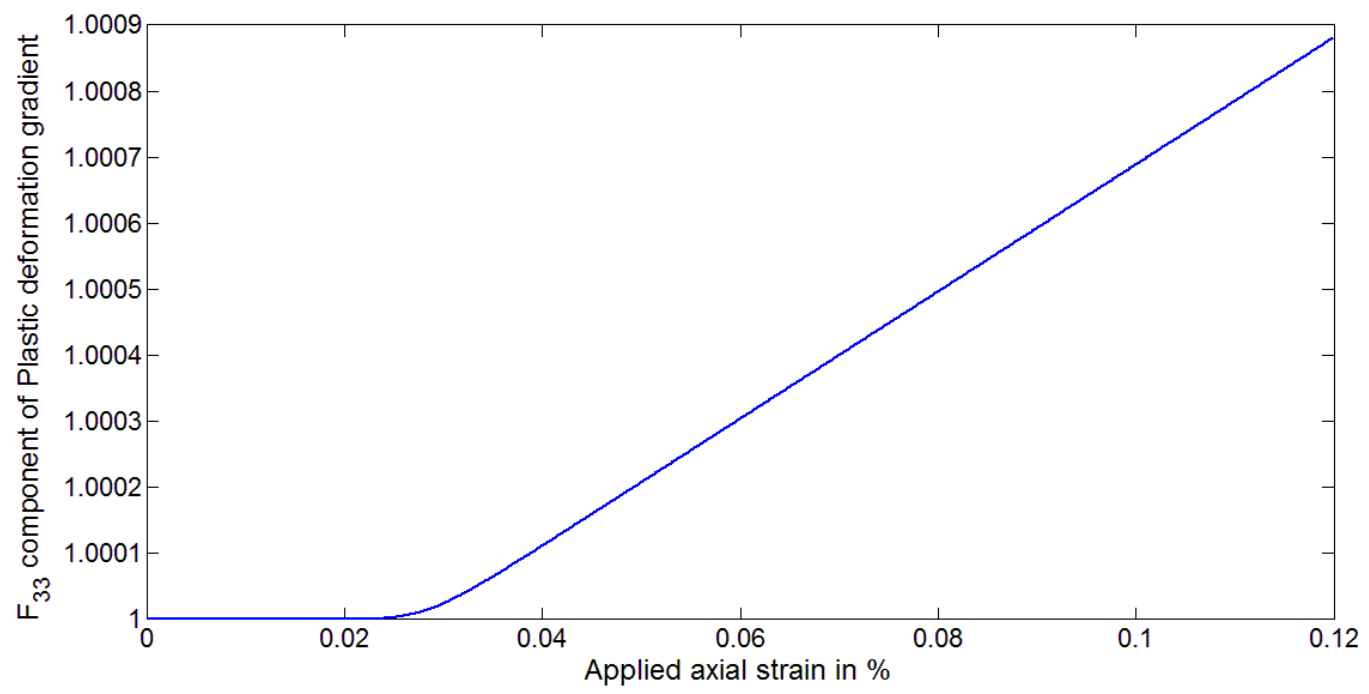

Figure 66 plot of $F_{p_{33}}$ vs time for the case of monotonic uniaxial tensile loading

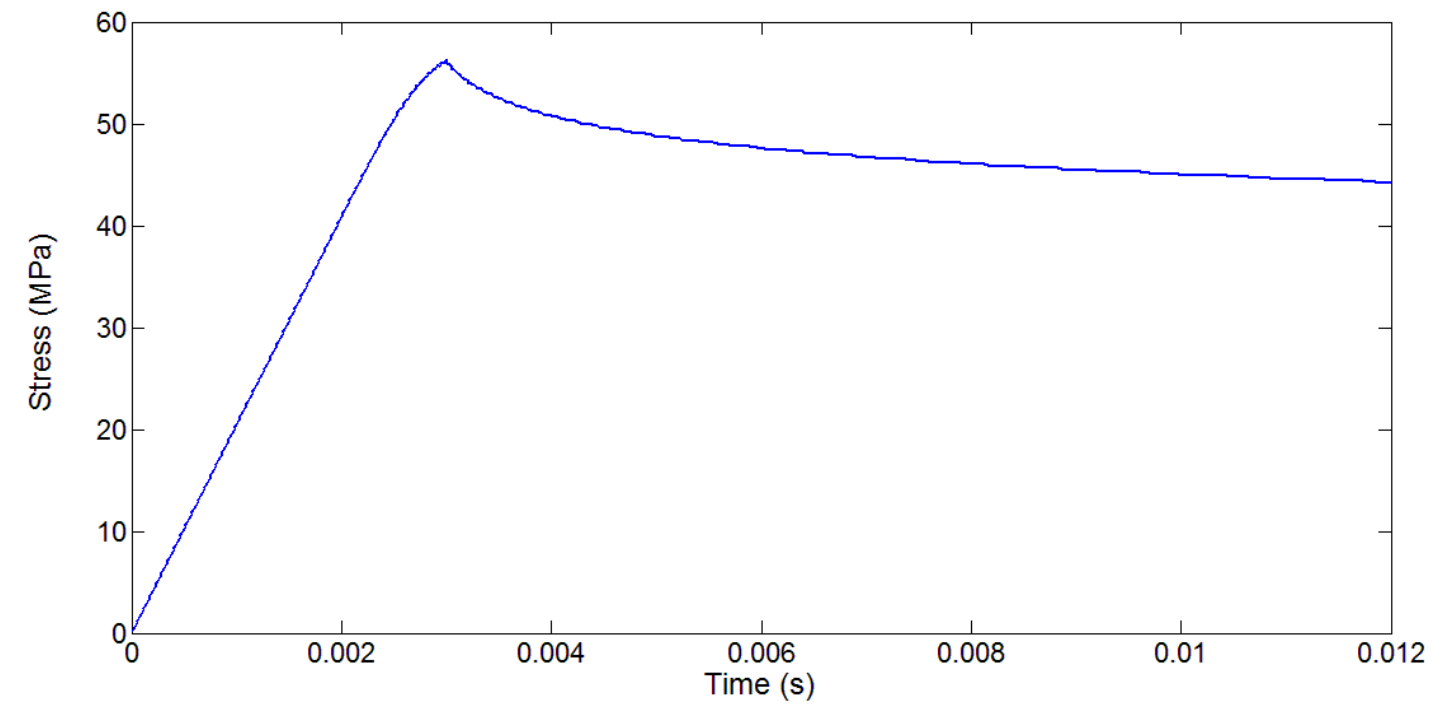

Figure 67 Average stress vs time plot for the case of elastic-plastic loading followed by relaxation 


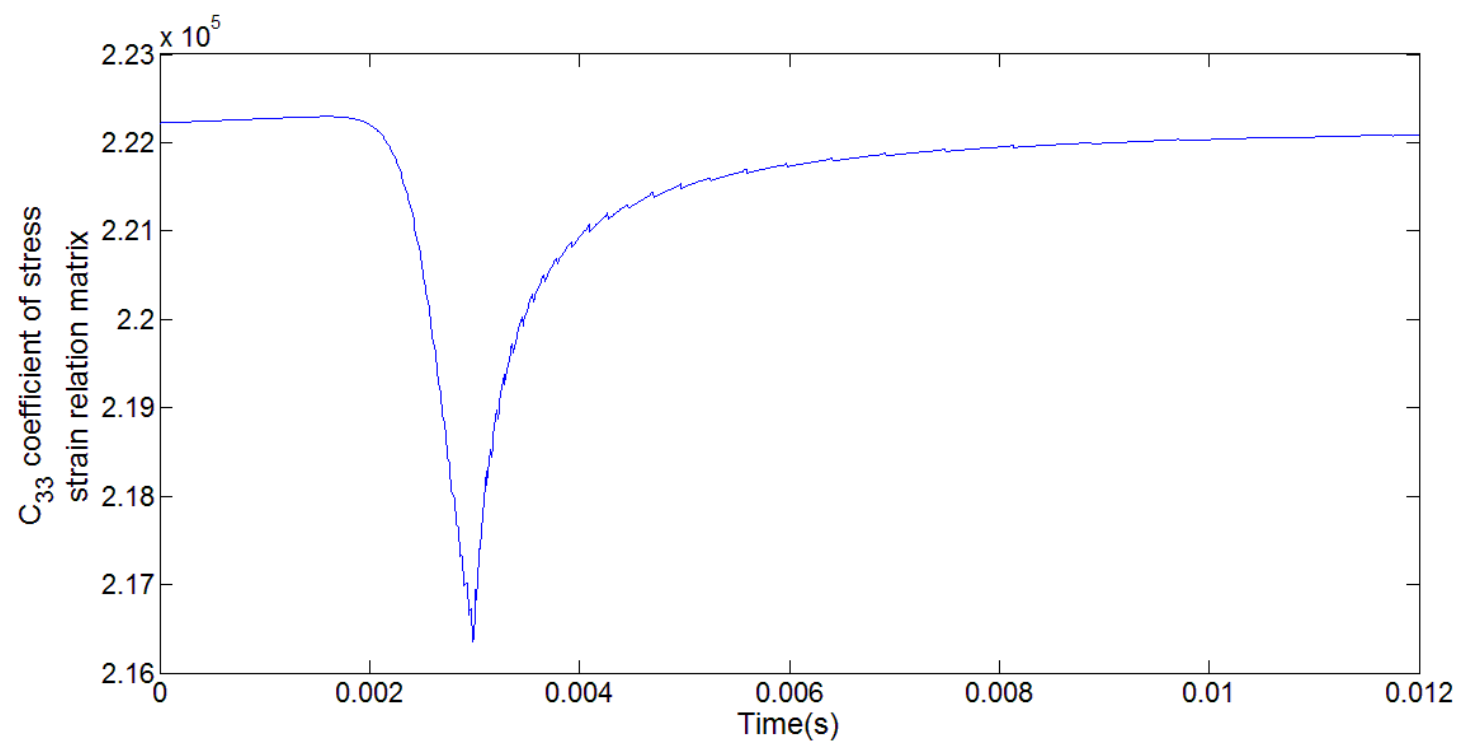

Figure 68 plot of $C_{33}$ (indicator of elastic modulus in the z-direction) vs time for the case of elastic-plastic loading followed by relaxation

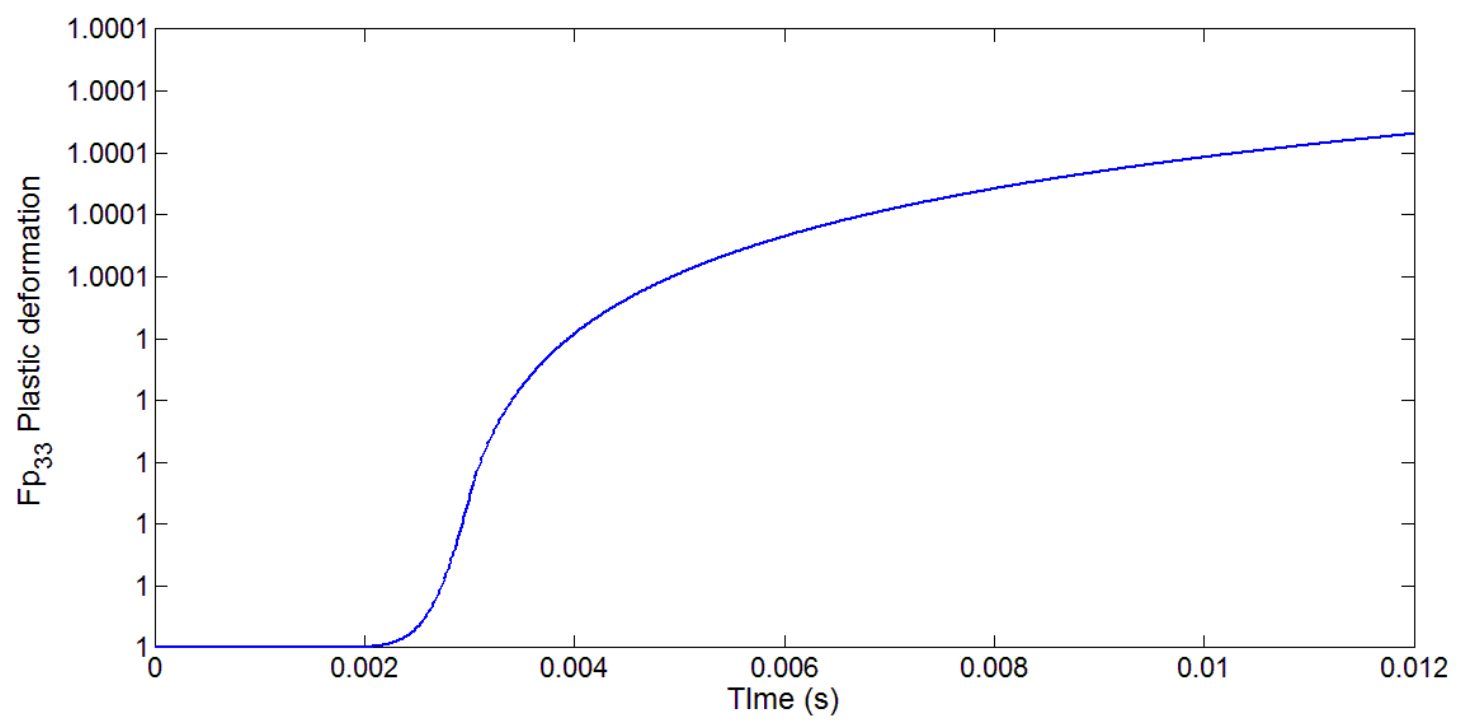

Figure 69 plot of $F_{p_{33}}$ vs time for the case of elastic-plastic loading followed by relaxation 

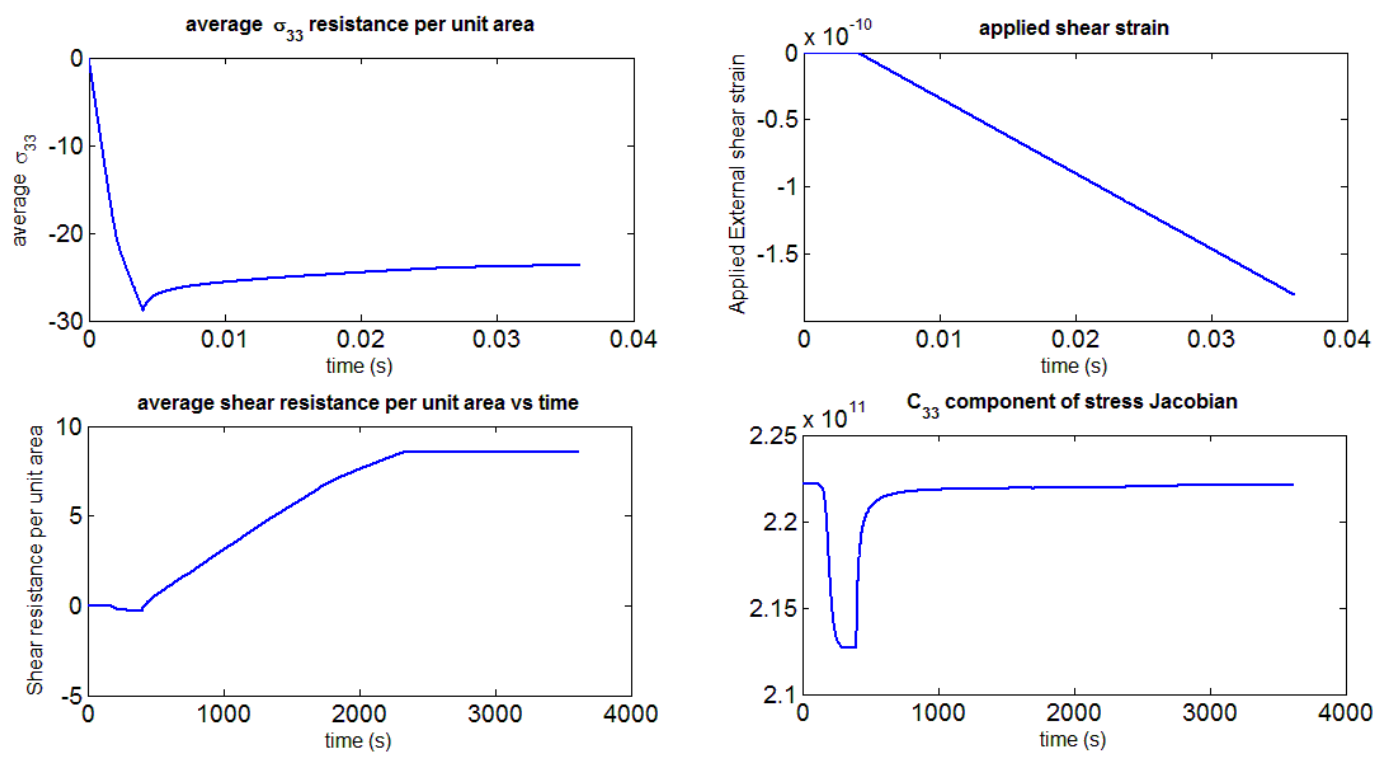

Figure 70 Plot of various quantities of interest in interface contact simulation with plasticity

A plot of various quantities of interest for interfacial contact simulation has been given in Figure 70.

\subsection{Conclusion}

A crystal plasticity constitutive model has been formulated. Simple case studies have been used to demonstrate the conceptual accuracy of the mechanics and physics involved in the present constitutive model formulation when combined with a nonlinear plasticity algorithm. It has been observed that the presented constitutive model and nonlinear plastic deformation algorithm are capable of simulating viscoplastic deformation of metals. Further the viscoplasticity has been incorporated in an interface friction simulation. For future work, addition of dislocation dynamics to the present model along with realistic boundary conditions will make it possible to simulate solid state manufacturing processes accurately. 


\section{CHAPTER 9}

\section{SUMMARY, CONCLUSIONS, AND FUTURE WORK}

\subsection{Summary}

The present work provides efficient algorithms with reduced computational complexity for simulating two classes of additive manufacturing processes: fusion based processes and solid state processes. These algorithms are validated with appropriate comparisons to established numerical algorithms such as the linear solver library of MATLAB and validated against various literature to determine their accuracy. A short summary of the present work for each class of processes is as follows.

\subsubsection{Fusion based processing simulations}

- The FFDAMRD numerical technique with adaptive meshing has significant potential for efficient and high resolution simulation of spatio-temporal problems with rapid time and spatial gradients. It has been established that traditional finite element approaches are 364.5times slower compared to the FFD-AMRD algorithm developed in this research for multi-scale moving mesh problems. This simulation algorithm can provide useful insights such as the temperature gradients shown in the case study, which are on the order of $10^{5} \mathrm{C} / \mathrm{s}$ at a laser speed of $1200 \mathrm{~mm} / \mathrm{s}$.

- The Eigenmodes formulation includes derivation of novel FEM based beam theories for thermal analysis of a general cross-section. This formulation offers 
multiple orders of magnitude speed improvement depending upon number of layers $(N)$ involved in the simulation. It has been established that for nearly identical cross-sections the computational speed improves by order of $(N)$. In the case of non-identical cross-sections this methodology works with at least the same complexity as that of FEM algorithms.

- The Cholesky decomposition algorithm has been modified based on observations that a major portion of Cholesky matrices involved in FEM are comprised of insignificantly small values. These small values are multiple orders of magnitude lower than computationally significant numbers. This algorithm reduces number of flops to between $5 \%$ and $30 \%$ of the flops that would typically be required, dependent on the boundary conditions involved.

- The work in Appendix A shows a nonlinear version of the FFD-AMRD algorithm presented in the present text. This algorithm has the ability to simulate temperature dependent material properties, latent heat and phase change effects for powder bed thermal behavior. The computational complexity of the Appendix A work and Chapter 4 work is the same since both are based on same algorithm except for the nonlinear material behavior being assumed linear in the Chapter 4 work.

\subsubsection{Solid state processing simulations}

- A new surface to surface contact simulation framework has been developed which solves constraint equations exactly without additional computational overhead. This formulation avoids usage of fictitious penalty springs which can induce spurious deformation modes in an analysis. It also includes an intelligent stiffness matrix assembly algorithm based on mapping points into their respective final 
location in the mesh. This formulation is capable of performing accurate contact simulations with the same or less computational cost than traditional contact algorithms.

- A crystal plasticity constitutive law has been developed with a tensorial analytical derivative of stress with respect to strain. A nonlinear plastic deformation algorithm works with the analytical deformation along with the novel contact simulation framework. This algorithm inherently simulates the viscoplastic deformation behavior of metals. This work provides a novel interface constitutive law which will reduce the computational cost of modeling interfaces along with bulk responses, resulting in a significantly simplified mesh and reduced computational cost.

\subsection{Conclusion}

In the present work new numerical algorithms have been formulated and implemented for simple additive manufacturing process simulation case studies. Each of these algorithms are at least an order of magnitude faster than traditional formulations and algorithms for FEM simulation. Conclusions derived from the present work are as follows.

- The developed dynamic meshing algorithm provides significant speed improvement for simulation of moving point or line energy sources such as in AM processes. It has been observed that these processes have multiple scales of response (greater than two) in space and time. 
- The modified Cholesky algorithm takes advantage of insight into asymptotics embedded in the Cholesky matrices with signficant potential for improved computational speed and data storage.

- An interfacial constitutive model formulation has been demonstrated that illustrates an efficient strategy to simulate energy dissipative mechanisms at interface between two layers in solid state bonding processes.

- The combination of the presented algorithms with various modal decomposition based techniques such as the beam theories developed in the present work has the potential to increase the computational speed by multiple orders of magnitude, making it possible to simulate AM processes in real time.

\subsection{Future work}

Algorithms in the present work offer a significant advantage over traditional ones used in FEM simulations. These algorithms can be further improved or augmented with other existing algorithms to increase computational speed and reduce computational complexity. Further work possible in this paradigm include:

- Incorporation of Eigenmode based beam theories with FFD-AMRD to simulate full powder bed problems. An alternate approach to this problem could be to couple analytical full powder bed solutions with FFDAMRD as discussed in a recent publication [144].

- To develop an analytical governing equation for Eigenmode based beam theories to be able to improve its applicability. 
- Addition of a dislocation density based constitutive model to the developed crystal plasticity simulation framework along with contact simulation. This will enable the simulation of any solid state AM process with significantly increased speeds with a physics based material constitutive model. This algorithm will be able to simulate texture and microstructural evolution during solid state processing. 


\section{REFERENCES}

[1] http://en.wikipedia.org/wiki/Fundamental_interaction

[2]Callister, W. D., \& Rethwisch, D. G. (2012). Fundamentals of materials science and engineering: an integrated approach. John Wiley \& Sons.

[3] https://en.wikipedia.org/wiki/Crystallographic_defect

[4] http://en.wikipedia.org/wiki/Quantum_mechanics

[5]Pal, D., Dislocation Density-Based Finite Element Method Modeling of Ultrasonic Consolidation

[6]El-Azab, A. "Statistical mechanics treatment of the evolution of dislocation distributions in single crystals." Physical Review B 61.18 (2000): 11956.

[7]Fish, Jacob, and Rong Fan. "Mathematical homogenization of nonperiodic heterogeneous media subjected to large deformation transient loading." International Journal for numerical methods in engineering 76, no. 7 (2008): 1044-1064.

[8]Chadwick, P. (1999). Continuum mechanics: concise theory and problems. Courier Dover Publications.

[9] Hughes, T. J. (2012). The finite element method: linear static and dynamic finite element analysis. Courier Dover Publications.

[10] Gibson, I., Rosen, D. W., \& Stucker, B. (2010). Additive manufacturing technologies: rapid prototyping to direct digital manufacturing. Springerverlag US. 
[11] Beeler D, Stucker B, Pal D, Patil N, Zeng K. 2012 AFRL report \# AFRL-rX-WP-TR2012-0510.

[12] ASTM. (2013), ASTM F2792 - 12a, Standard terminology for additive manufacturing technologies, Retrieved from www.ASTM.org/Standards/F2792.htm.

[13] Dutta D. , Prinz F.B., Rosen D., and Weiss L., (2000), Layered Manufacturing: Current Status and Future Trends, Journal of Computing and Information Science in Engineering (ASME), Volume 1, pp.60-71

[14] Santos E. C., Shiomi M., Osakada K., and Laoui T., (2006),Rapid Manufacturing of Metal Components by Laser Forming, International Journal of Machine Tool and Manufacture, Volume 46, pp.1459-1468

[15] Levy G.N., Schindel R., and Kruth J.P., (2003), Rapid Manufacturing and Rapid Tooling with Layer Manufacturing (LM) Technologies, State of the Art and future Perspectives, CIRP Annals - Manufacturing Technology, Volume 52, pp.589-609

[16] Karapatis N.P., Van Griethuysen J.P.S., Glardon R., (1995), Direct Rapid Tooling: A Review of Current Research, Rapid Prototyping Journal, Volume 4, pp.77-89.

[17] Bohrer M., Basalka H., Birner W., Emiljanow K., Goede M., and Czerner S., Turbine Blade Repair With Laser Fusion Welding and Shape Recognition, Proceedings of the 2002 International Conference on Metal Powder Deposition for Rapid Manufacturing.

[18] Stevens B., Yang Y., Mohandas A., Stucker B.E., and Nguyen K., (2007), A Review of Materials, Fabrication Methods, and Strategies Used to Enhance Bone Regeneration Engineered Bone Tissues, Journal of Biomedical Materials Research: Part B - Applied Biomaterials, Volume 85, pp.573-582. 
[19] Cohen D.L., Malone E., Lipson H., andBonassar L.J., (2006), Direct Freeform Fabrication of Seeded Hydrogels in Arbitrary Geometries, Tissue Engineering, Volume 12, pp.1325-1335.

[20] Wu B.M., Borland S.W., Giordano R.A., (1996), Cima L.G., Sachs E.M., and Cima M.J., Solid free-form fabrication of drug delivery devices, Journal of Controlled Release, Volume 40, pp.77-87.

[21] Hong S.B., Eliaz N., Sachs E.M., Allen S.M., and Latanisan R.M., (2001). Corrosion behavior of advanced titanium-based alloys made by three-dimensional printing (3DPTM) for biomedical applications, Corrosion Science, Volume 43, pp.1781-1791

[22] Gibson, L., Rosen, D., Stucker, B. (2009), "Additive manufacturing technologies: rapid prototyping to direct digital manufacturing", Springer, New York, NY

[23] X. C. Wang, T. Laoui, et al. (2002), "Direct Selective Laser Sintering of Hard Metal Powders:Experimental Study and Simulation", Int J AdvManufTechnol, Vol.19 pp.351357

[24] J.P. Kruth, X. Wang, et al. (2003),"Lasers and materials in selective laser sintering",Assembly Automation, Vol. 23 Iss: 4 pp. 357 - 371

[25] Simchi, A. (2006). "Direct laser sintering of metal powders: Mechanism, kinetics and microstructural features.", Materials Science and Engineering: A 428, Vol. 1 iss:2 pp.148158.

[26] Kolossov, S., E. Boillat, et al. (2004). "3D FE simulation for temperature evolution in the selective laser sintering process." International Journal of Machine Tools and Manufacture, Vol. 44 iss: 2-3 pp.117-123. 
[27] Zhang, D. Q., Q. Z. Cai, et al. (2010). "Select laser melting of W-Ni-Fe powders: simulation and experimental study." The International Journal of Advanced Manufacturing Technology, Vol. 51 iss:5-8 pp. 649-658.

[28] Contuzzi,N.,Campanelli,S.L., et al. (2011), "3d Finite element analysis in the selective laser

melting process",Int j simul model, Vol. 10 iss:3 pp.113-121

[29] Song, Y. (1997). Experimental Study of the Basic Process Mechanism for Direct Selective Laser Sintering of Low-Melting Metallic Powder. CIRP Annals-Manufacturing Technology, 46(1), 127-130

[30] Zeng, K., Pal, D., \& Stucker, B. A review of thermal analysis methods in Laser Sintering and Selective Laser Melting.

[31] Kruth, J.P., Levy, G. et al., (2007), Consolidation phenomena in laser and powder-bed based layered manufacturing, Original Research Article CIRP Annals - Manufacturing Technology 2007;56:730.

[32] Levy, G.N., Schindel, R., et al., (2003), Rapid manufacturing and rapid tooling with layer manufacturing $(\mathrm{lm})$ technologies, state of the art and future perspectives, CIRP Annals - Manufacturing Technology 2003;52:589.

[33] Miani F. Recent Developments Of Direct Metal Selective Laser Sintering", available at: http://www.ics.trieste.it/media/133856/df143.pdf (accessed 2 August 2012).

[34] J.P. Kruth, B. Vandenbroucke, et al, "Benchmarking Of Different Sls/Slm Processes As Rapid Manufacturing Techniques", In Int. Conf. Polymers \&Moulds Innovations (PMI), Gent, Belgium, April 20-23, 2005 
[35] Sanjay Kumar(2003), "Selective Laser Sintering:A Qualitative and Objective Approach", JOM Journal of the Minerals, Vol.55, iss:10 pp.43-47

[36] J.P. Kruth, X. Wang, et al. (2003), "Lasers and materials in selective laser sintering", Assembly Automation, Vol. 23 Iss: 4, pp.357 - 371

[37] Nikolay K. Tolochko, et al. (2003),"Mechanisms of selective laser sintering and heat transfer in Ti powder", Rapid Prototyping Journal, Vol. 9 Iss: 5 pp. 314-326.

[38] X. C. Wang, T. Laoui, et al. (2002), "Direct Selective Laser Sintering of Hard Metal

[39] Simchi, A. (2006). "Direct laser sintering of metal powders: Mechanism, kinetics and microstructural features.", Materials Science and Engineering: A 428, Vol. 1 iss:2 pp.148158.

[40] Roberts IA, Wang CJ et al. International Journal of Machine Tools and Manufacture 2009;49:916.

[41] Fischer, P., V. Romano, et al. (2003). "Sintering of commercially pure titanium powder with a Nd:YAG laser source." ActaMaterialia, Vol. 51 iss:6 pp. 1651-1662.

[42] Singh, S. S., D. Roy, et al. (2009). "Studies on laser sintering of mechanically alloyed Al50Ti40Si10 composite." Materials Science and Engineering,Vol.501 iss:1 pp. 242-247

[43] J.P. Kruth, X. Wang, et al. (2003),"Lasers and materials in selective laser sintering",Assembly Automation, Vol. 23 Iss: 4 pp. 357 - 371

[44] Pal, D., (2011), Dislocation Density-Based Finite Element Method Modeling of Ultrasonic Consolidation, PhD thesis, Utah State University, Logan, Utah.

[45] Kong C.Y., Soar R.C., Dickens P.M., (2004), Optimum Process Parameters for Consolidation of 3003 Aluminum, Journal of Materials Processing Technology, Volume 146, pp.181-187. 
[46] Janaki Ram G.D., Yang and Stucker B.E., (2006), Effects of Process Parameters on Bond Formation During Ultrasonic Consolidation of Aluminum Alloy 3003, Journal of Manufacturing Systems, Volume 25, pp.221-238.

[47] Obielodan J.O., Janaki Ram G.D., Stucker B.E. and Taggart D.G., (2009), Minimizing Defects Between Adjacent Foils in Ultrasonically Consolidated Parts, Journal of Engineering Materials and Technology, Volume 132, pp.011006.1-8

[48] Kong C.Y., Soar R.C, Dickens P.M., (2004). Ultrasonic Consolidation for Embedding SMA fibers within Aluminium Matrices, Composite Structures, Volume 66, pp.421-427. [49] Kong C.Y., and Soar R.C., (2005), Fabrication of Metal-Matrix Composites and Adaptive Composites Using Ultrasonic Consolidation process, Materials Science and Engineering A, Volume 412, pp.12-18.

[50] Yang Y., Janaki Ram G.D., and Stucker B.E., (2007), An Experimental Determination of Optimum Processing Parameters for $\mathrm{Al} / \mathrm{SiC}$ Metal Matrix Composites Made Using Ultrasonic Consolidation, Journal of Engineering Materials and Technology, Volume 129, pp.538-549.

[51] Yang Y., Janaki Ram G.D., and Stucker B.E., (2009), Bond Formation and Fiber Embedment during Ultrasonic Consolidation, Journal of Materials Processing Technology, Volume 209, pp.4915-4924.

[52] Obielodan J.O. and Stucker B.E., (2009), Effects of Post Processing Heat Treatments on the Bond Quality and Mechanical Strength of Ti/Al 3003 Dual Materials Fabricated using Ultrasonic Consolidation, Proceedings of Solid Freeform fabrication Symposium, Austin, TX, USA, Aug. 2009. 
[53] Janaki Ram G.D., Yang and Stucker B.E., (2006), Effects of Process Parameters on Bond Formation During Ultrasonic Consolidation of Aluminum Alloy 3003, Journal of Manufacturing Systems, Volume 25, pp.221-238.

[54] Obielodan J.O., Janaki Ram G.D., Stucker B.E. and Taggart D.G., (2009), Minimizing Defects Between Adjacent Foils in Ultrasonically Consolidated Parts, Journal of Engineering Materials and Technology, Volume 132, pp.011006.1-8

[55] Kong C.Y., Soars R.C., and Dickens P.M., (2003), Characterization of aluminium alloy 6061 for the ultrasonic consolidation process, Materials Science and Engineering A, Volume 363, pp.99-106.

[56] Gonzalez, R., and Brent Stucker. Experimental Determination of Optimum Parameters for 316/316L Stainless Steel Ultrasonic Consolidation, Solid Freeform Fabrication Symposium, Austin, TX, USA, August 2009.

[57] Berger M, Oliger J., (1984), Adaptive mesh refinement for hyperbolic partial differential wquations, J. Comput. Phys, vol 53, 484-512.

[58] Berger MJ, Colella P., (1989), Local Adaptive Mesh Refinement for Shock Hydrodynamics, ournal of Computational Physics, vol 82, 64-84.

[59] Carey G. Computer Methods in Applied Mechanics and Engineering2006;195:214.

[60] T. Grätsch and K.J. Bathe, (2005), "A posteriori Error Estimation Techniques in Practical Finite Element Analysis", Computers \& Structures, 83, 235-265, 2005 [61] http://www.cs.berkeley.edu/ demmel/cs267_Spr06/Lectures/Lecture24/Lecture_24_AM $\underline{\text { R_Colella_06.pdf }}$ 
[62] Lax, P. D.; Richtmyer, R.D., (1956), Survey of the stability of linear finite difference equations. Comm. Pure Appl. Math. 9 (1956), 267-293

[63] Akin, J.K., Finite Element analysis with error estimates, Academic Pr; 1994.

[64] Zienkiewicz, O.C., Zhu, J.Z., (1992), Thesuperconvergent patch recovery and a posteriori error estimates - Part 1: the technical recovery" - Int. Newspaper for Num. Puts. in Eng., flight $33.1331-1364$.

[65] www.cs.rpi.edu/ flaherje/pdf/fea8.pdf, Chapter 8 Adaptive Finite Element Refinement

[66] Y. Liu, C. D. Sarris, Dynamically Adaptive Mesh Refinement FDTD: A stable and efficient technique for time-domain simulations, Proc. 12th International Symposium on Antenna Technology and Applied Electromagnetics and URSI/CNC Conference, , QC, Canada, 2006, pp. 621-624.

[67] Ann S, Almgren, CCSE Berkley Presentation Introduction to Block-Structured Adaptive Mesh Refinement (AMR) hipacc.ucsc.edu/.../AlmgrenHIPACC_July2011.pdf [68] B. Delaunay: Sur la sphère vide, IzvestiaAkademiiNauk SSSR, OtdelenieMatematicheskikhiEstestvennykhNauk, 7:793-800, 1934

[69] Bank, R.E., Sherman, A.H., Weiser, A., (1983), Refinement algorithms and data structures for regular local mesh renement, IMACS Transactions on Scientific Computing 1983;1:3.

[70] Aiffa, M., Adaptive hpRefinement Methods for SingularlyPerturbed Elliptic and Parabolic Systems. PhD thesis, Rensselaer Polytechnic Institute, Troy. 
[71] Smith MJ, Hodges DH, Cesnik CES. An Evaluation of Computational Algorithms to Interface Between CFD and CSD Methodologies, WL-TR- 96-3055. Technical report, Flight Dynamics Directorate, Wright Laboratory, Wright-Patterson Air Force Base 1995. [72] Ahmed F. Analysis of Data Transfer Methods Between Non-Matching Meshes In Multiphysics Simulations, Universität Erlangen-Nürnberg.

[73] Wendland H. J. Approx. Theory : Wavelets, Splines, and Applications. 2002; pp 473; [74] Beckert A, Wendland H., (2001), Multivariate Interpolation for FluidStructureInteraction Problems Using Radial Basis Function, Aerosp. Sci. Technolvol 5, 111.

[75] Wendland, H., (2005), Computational Aspects of Radial Basis Functions Approximation. In Jetter $\mathrm{K}$ et al., editor, Topics in Multivariate Approximation and Interpolations, pages 1-25. Elsevier.

[76] Murti, V., and Valliappan, S., (1986), Numerical Inverse Isoparametric Mapping inRemeshing and Nodal Quantity Contouring. Computers and Structures, 22(6):10111021.

[77] Fish, Jacob, and Rong Fan. "Mathematical homogenization of nonperiodic heterogeneous media subjected to large deformation transient loading." International Journal for numerical methods in engineering 76, no. 7 (2008): 1044-1064.

[78] M. Berger, J. Oliger, Adaptive mesh refinement for hyperbolic partial differential equations, Journal of Computational Physics 53 (3) (1984) 484-512.

[79] M. Berger, P. Colella, Local Adaptive Mesh Refinement for Shock Hydrodynamics, Journal of Computational Physics 82 (1) (1989) 64-84. 
[80] G. Carey, A perspective on adaptive modeling and meshing (AM\&M), Computer Methods in Applied Mechanics and Engineering 195 (4-6) (2006) 214-235.

[81] T. Grätsch, J. Bathe, A posteriori Error Estimation Techniques in Practical Finite Element Analysis, Computers \& Structures 83, 235 (2005).

[82] P. Lax, A. Milgram, "Parabolic equations". Contributions to the theory of partial differential equations. Annals of Mathematics Studies, Annals of Mathematics Studies 33 (1954) 167-190.

[83] J. E. Akin, Finite Finite element analysis with error estimators: An introduction to the FEM and adaptive error analysis for engineering students, Butterworth-Heinemann, 2005, pp 127-143.

[84] O. C. Zienkiewicz, J. Z. Zhu, The superconvergent patch recovery and a posteriori error estimates - Part 1: the technical recovery, International Journal for Numerical Methods in Engineering 33 (7) (1992) 1331-1364.

[85] Y. Liu, C. D. Sarris, Dynamically Adaptive Mesh Refinement FDTD: A stable and efficient technique for time-domain simulations, Proc. 12th International Symposium on Antenna Technology and Applied Electromagnetics and URSI/CNC Conference, , QC, Canada, 2006, pp. 621-624.

[86] J. A. F. Hittinger, J. W. Banks, Block-structured adaptive mesh refinement algorithms for Vlasov simulation, Journal of Computational Physics 241 (1) (2013) 118-140.

[87] H. Zhang, V. Prasad, M. K. Moallemi, Numerical algorithm using multizone adaptive grid generation for multiphase transport processes with moving and free boundaries, Numerical Heat Transfer, Part B: Fundamentals: An International Journal of Computation and Methodology, 29 (4) (1996) 399-421. 
[88] W. Huang, Y. Ren, R. Russell, Moving Mesh Partial Differential Equations (MMPDES) Based on the Equidistribution Principle, Siam Journal of Numerical Analysis 31 (3) (1994) 709-730.

[89] M. Smith, D. Hodges, C. Cesnik , An evaluation of computational algorithms to interface between CFD and, CSD methodologies, Wright-Patterson Air Force Base Report No. WL-TR- 96-3055, 1995, pp. 1-45.

[90] F. Ahmed, Analysis of Data Transfer Methods Between Non-Matching Meshes In Multiphysics Simulations, M.S. Thesis, Universität Erlangen-Nürnberg, ErlangenNürnberg, 2006.

[91] H. Wendland, Fast Evaluation of Radial Basis Functions: Methods Based on Partition of Unity, Approximations Theory X: Wavelets, Splines, and Applications. Vanderbilt University Press, 2002, pp. 473-483.

[92] A. Beckert, H. Wendland, Multivariate Interpolation for Fluid-Structure Interaction Problems Using Radial Basis Function, Aerospace Science and Technology, 5 (2) (2001) $125-134$.

[93] H. Wendland., Computational Aspects of Radial Basis Function Approximation, Studies in Computational Mathematics 12 (2006) 231-256.

[94] T. Chandrupatla, A. Belegundu, Introduction to finite elements in engineering, (NJ: Prentice-Hall, 1991.

[95] V. Murti, S. Valliappan, Numerical Inverse Isoparametric Mapping inRemeshing and Nodal Quantity Contouring, Comput. Struct. 22 (6) (1986) 1011-1021.

[96] Ceniceros, H. D., \& Hou, T. Y. (2001). An efficient dynamically adaptive mesh for potentially singular solutions. Journal of Computational Physics, 172(2), 609-639 
[97] Adjerid, S., \& Flaherty, J. E. (1986). A moving finite element method with error estimation and refinement for one-dimensional time dependent partial differential equations. SIAM Journal on Numerical Analysis, 23(4), 778-796.

[98] Liao, G., Liu, F., De la Pena, G. C., Peng, D., \& Osher, S. (2000). Level-set-based deformation methods for adaptive grids. Journal of Computational Physics, 159(1), 103122.

[99] Hyman, J. M., \& Li, S. (1998). Interactive and dynamic control of adaptive mesh refinement with nested hierarchical grids. Rea port, (5462).

[100] Wang, L., \& Moriwaki, T. (2003). A novel meshing algorithm for dynamic finite element analysis. Precision engineering, 27(3), 245-257.

[101] Boussetta, R., Coupez, T., \& Fourment, L. (2006). Adaptive remeshing based on a posteriori error estimation for forging simulation. Computer methods in applied mechanics and engineering, 195(48), 6626-6645.

[102] Matsumoto, M., Shiomi, M., Osakada, K., \& Abe, F. (2002). Finite element analysis of single layer forming on metallic powder bed in rapid prototyping by selective laser processing. International Journal of Machine Tools and Manufacture, 42(1), 61-67.

[103] Shiomi, M., Yoshidome, A., Abe, F., \& Osakada, K. (1999). Finite element analysis of melting and solidifying processes in laser rapid prototyping of metallic powders. International Journal of Machine Tools and Manufacture,39(2), 237-252.

[104] Verhaeghe, F., Craeghs, T., Heulens, J., \& Pandelaers, L. (2009). A pragmatic model for selective laser melting with evaporation. Acta Materialia, 57(20), 6006-6012.

[105] Zaeh, M. F., \& Branner, G. (2010). Investigations on residual stresses and deformations in selective laser melting. Production Engineering, 4(1), 35-45. 
[106] Dai, K., \& Shaw, L. (2004). Thermal and mechanical finite element modeling of laser forming from metal and ceramic powders. Acta Materialia, 52(1), 69-80.

[107] Kolossov, S., Boillat, E., Glardon, R., Fischer, P., \& Locher, M. (2004). 3D FE simulation for temperature evolution in the selective laser sintering process.International Journal of Machine Tools and Manufacture, 44(2), 117-123.

[108] Roberts, I. A., Wang, C. J., Esterlein, R., Stanford, M., \& Mynors, D. J. (2009). A three-dimensional finite element analysis of the temperature field during laser melting of metal powders in additive layer manufacturing. International Journal of Machine Tools and Manufacture, 49(12), 916-923.

[109] Hussein, A., Hao, L., Yan, C., \& Everson, R. (2013). Finite element simulation of the temperature and stress fields in single layers built without-support in selective laser melting. Materials \& Design.

[110] Childs, T. H. C., Hauser, C., \& Badrossamay, M. (2005). Selective laser sintering (melting) of stainless and tool steel powders: experiments and modelling. Proceedings of the Institution of Mechanical Engineers, Part B: Journal of Engineering Manufacture, 219(4), 339-357.

[111] Kolossov, S., Boillat, E., Glardon, R., Fischer, P., \& Locher, M. (2004). 3D FE simulation for temperature evolution in the selective laser sintering process.International Journal of Machine Tools and Manufacture, 44(2), 117-123.

[112] D. Pal, N. Patil, K. Rafi, K. Zeng, A. Moreland, A. Hicks, D. Beeler, B. Stucker, A Feed Forward Dynamic Adaptive Mesh Refinement and De-refinement (FFD-AMRD) strategy for problems with non-linear spatiotemporally periodic localized boundary conditions, Journal of Applied Physics. xxx, xx (2013) xx-xx. 
[113] G. P. Nikishkov, Programming Finite Elements in JavaTM, Springer, 2010, pp. 1319.

[114] D. Beeler, B. Stucker, D. Pal, N. Patil, K. Zeng, Computational Modeling of Ti6Al4V alloy processed using Direct Metal Laser Sintering Process, Air Force Research Laboratory Report No. AFRL-rX-WP-TR-2012-0510, 2012.

[115] L. Trefethen, D. Bau, Numerical linear algebra (Society for Industrial and Applied Mathematics, 1997, pp. 175.

[116] Zeng, K., Pal, D., \& Stucker, B. A review of thermal analysis methods in Laser Sintering and Selective Laser Melting, Solid Freeform Fabrication Symposium 2012, Texas Austin.

[117] Patil, N., Pal, D., Rafi, K., Zeng, K., Beeler, D., \& Stucker, B., “A generalized feed forward dynamic adaptive mesh refinement and de-refinement finite element framework for spatiotemporally periodic localized boundary conditions: Application to Metal Laser Sintering", International Journal of Thermal Sciences, $\operatorname{xxx}, \operatorname{xxxxxx}$ (2013). (in communication).

[118] Pal, D., Patil, N., Rafi, K., Zeng, K., Beeler, D., \& Stucker, B., “A Feed Forward Dynamic Adaptive Mesh Refinement and De-refinement (FFD-AMRD) strategy for problems with non-linear spatiotemporally periodic localized boundary conditions", J. Journal of Applied Physics, xxx, xxxxxx (2013). (in review).

[119] Mumtaz, K., \& Hopkinson, N. (2010). Selective laser melting of Inconel 625 using pulse shaping. Rapid Prototyping Journal, 16(4), 248-257.

[120] Nikishkov, G.P., Programming Finite Elements in JavaTM, Springer, 2010, pp. 1319. 
[121] Crank, J. \& Nicolson, P. (1947). "A practical method for numerical evaluation of solutions of partial differential equations of the heat conduction type". Proc. Camb. Phil. Soc. 43 (1): 50-67.

[122] Rafi, H., Pal, D., Patil,N., Starr, T., \& Stucker, B., Microstructure and mechanical behavior of 17-4 precipitation hardenable stainless steel processed by Selective Laser Melting, (In preparation).

[123] Berdichevsky, V. L., "Variational-Asymptotic Method of Constructing a Theory of Shells," PMM , Vol. 43, No. 4, 1979, pp. $664-687$.

[124] Li, Xiaoye S. "An overview of SuperLU: Algorithms, implementation, and user interface." ACM Transactions on Mathematical Software (TOMS) 31.3 (2005): 302-325. [125] Golub, Gene H., and Charles F. Van Loan. "Matrix computations. 1996." Johns Hopkins University, Press, Baltimore, MD, USA (1983).

[126] Berry, K. J. "An efficient C-based wavefront solver for PC finite element applications." Computers \& Structures 39.3 (1991): 303-315.

[127] Ikram, Muhammad Umer. Application Specific Precision Analysis of Cholesky Decomposition in MMSE MIMO Receiver Systems. Diss. 2010.

[128] Gill, P. E., \& Murray, W. (1974). Newton-type methods for unconstrained and linearly constrained optimization. Mathematical Programming, 7(1), 311-350.

[129] Jones, M. T., \& Plassmann, P. E. (1995). An improved incomplete Cholesky factorization. ACM Transactions on Mathematical Software (TOMS), 21(1), 5-17.

[130] Menon, V., \& Pingali, K. (2004). Look left, look right, look left again: An application of fractal symbolic analysis to linear algebra code restructuring.International Journal of Parallel Programming, 32(6), 501-523. 
[131] Karmarkar, N. (1990). An interior \{point approach to NP \{complete problems| part I. Contemporary Mathematics, 114, 297-308.

[132] Sun, J. G. (1992). Rounding-error and perturbation bounds for the Cholesky and $\mathrm{LDL}^{\mathrm{T}}$ factorizations. Linear algebra and its applications, 173, 77-97.

[133] Pal, D., (2011), Dislocation Density-Based Finite Element Method Modeling of Ultrasonic Consolidation, PhD thesis, Utah State University, Logan, Utah.

[134] Pal, D., Patil, N., \& Stucker, B. (2013). A study of subgrain formation in Al 3003 H18 foils undergoing ultrasonic additive manufacturing using a dislocation density based crystal plasticity finite element framework. Journal of Applied Physics,113, 203517.

[135] Barenblatt, G. I., (1962). The mathematical theory of equilibrium cracks in brittle fracture, Advances in Applied Mechanics, 7(1), 55-129.

[136] Dugdale, D. (1960). Yielding of steel sheets containing slits. Journal of the Mechanics and Physics of Solids, 8(2), 100-104.

[137] Su, C., Wei, Y.J., \& Anand, L., (2004), An elastic-plastic interface constitutive model: application to adhesive joints, International Journal of Plasticity 20(12), 20632081.

[138] Dolbow, J. O. H. N., \& Belytschko, T. (1999). A finite element method for crack growth without remeshing. Int. J. Numer. Meth. Engng, 46, 131-150.

[139] Patil, N., Pal, D., \& Stucker, B. E. (2013). An Energy Dissipative Constitutive Model for Multi-Surface Interfaces at Weld Defect Sites in Ultrasonic Consolidation. Proceedings of the Solid Freeform Fabrication Symposium.

[140] http://www.kochmann.caltech.edu/research_finiteplasticity.html [141] http://www.kochmann.caltech.edu/research_finiteplasticity.html 
[142]Balasubramanian, S. (1998). Polycrystalline plasticity: application to deformation processing of lightweight metals (Doctoral dissertation, Massachusetts Institute of Technology).

[143] Wu, P. D., Neale, K. W., \& Van der Giessen, E. (1996). Simulation of the behaviour of FCC polycrystals during reversed torsion. International Journal of Plasticity, 12(9), 1199-1219.

[144] Pal, D., Patil, N., Nikoukar, M., Zeng, K., Kutty, K. H., \& Stucker, B. E. An Integrated Approach to Cyber-Enabled Additive Manufacturing using Physics based, Coupled Multi-scale Process Modeling. 


\title{
APPENDIX A
}

\section{A GENERALIZED FEED FORWARD DYNAMIC}

\author{
ADAPTIVE MESH REFINEMENT AND DE-
}

REFINEMENT FINITE ELEMENT FRAMEWORK FOR

\section{METAL LASER SINTERING: PART II (NON-LINEAR}

\section{THERMAL SIMULATIONS AND VALIDATIONS)}

Deepankar Pal ${ }^{1, \mathrm{a}}$, Nachiket Patil ${ }^{\mathrm{a}}$, Khalid Rafi ${ }^{\mathrm{a}}$, Kai Zeng ${ }^{\mathrm{a}}$, Alleyce Moreland ${ }^{\mathrm{b}}$, Adam Hicks $^{\mathrm{b}}$, David Beeler ${ }^{\mathrm{b}}$ and Brent E. Stucker ${ }^{\mathrm{a}}$

${ }^{a}$ Department of Industrial Engineering, University of Louisville, Louisville, KY 40292.

${ }^{\mathrm{b}}$ Mound Laser and Photonics Center, Kettering, OH 45420.

\section{A.1 Introduction}

Metal laser sintering is a layer-by-layer additive manufacturing process that uses 3D CAD data in the form of a stl file as a digital information source and energy in the

\footnotetext{
${ }^{1}$ Corresponding author at:

Department of Industrial Engineering, University of Louisville, Louisville, KY 40292, USA

Email address: d0pal001@ louisville.edu

Phone: 502-852-2542
} 
form of a high powered laser beam to create three-dimensional metal parts by fusing fine metallic powders together. A common setup is comprised of a fiber laser heat source and a powder bed held over a base plate. The laser scans on top of the powder bed, following a prescribed scan pattern, to melt a layer of powdered material based upon the .stl file.

The layer-by-layer addition of material and selective laser exposure of the powder bed leads to fabrication of individual parts with complex geometries. One of the major advantages of metal laser melting is the elimination of part-specific tooling and preproduction costs. The process is also versatile in nature since a variety of metals and their constituent alloys such as steels, aluminum, titanium and nickel based superalloys can be used for fabrication. Due to its ability to fully melt the exposed regions of the powder bed, close to full density-parts are generally fabricated using metal laser sintering [145].

Parts fabricated using the metal laser sintering process possess salient features such as complex geometries and structures with thin walls and hidden voids or channels. For instance, fully dense solid and porous lattice structures can be produced in the same part to enable complex biomedical implants such as a hip stem or a knee cup to be produced. Apart from the biomedical arena, other areas of application are in fabrication of lightweight parts for aerospace applications where manufacturing constraints such as tooling, or cutting tool access for material removal and machining leads to significant limitations in design [146].

Any manufacturing process requires its physics to be understood in order to optimize it. Since metal laser sintering involves melting and fusion of fine metallic powders, understanding thermal evolution during the process is of great interest: thermal history drives microstructural evolution, and microstructure and phase morphology 163 
influence in-service performance and longevity of the fabricated part. Many researchers have put efforts toward understanding the process [147-151] and formulating models to describe thermal evolution [152-157] accurately. Simulation models proved beneficial for demonstrating the influence of various input processing parameters. These models provide essential tools for identifying optimal parameter combinations without extensive testing [157]. The heat transfer mechanisms consist of powder bed radiation, convection between the powder bed and environment, and heat conduction inside the powder bed and between the powder bed and substrate (or prior layers). The heat transfer mechanisms also involve a huge latent heat of fusion since the process involves full melting of portions of the powder bed. Complex metallurgical phase changes that occur during processing complicate the heat transfer problem as thermophysical properties have a complex, non-linear relationship with temperature [158].

\section{A.2. Problem Description}

\section{A.2.1 Metal laser sintering of Ti6Al4V}

The three dimensional spatio-temperally periodic geometry, boundary conditions and material behavior described below represent a mathematical description of the metal laser sintering problem.

\section{A.2.1.1 Reference Geometry and Material Description}

The reference geometry considered here is shown in Figure A1. A thermally nonlinear, homogenous and isotropic material behavior has been assumed for the Ti6Al4V material during processing. The powder and solidified regions have been assumed to be

distinct homogenous entities. The geometry of the problem is a solid base plate of 
thickness $25.4 \mathrm{~mm}$ and a powder layer of thickness $30 \mu \mathrm{m}$. The temperature dependent non-linearity in thermal parameters is the same as that of a bulk Ti6Al4V material [159] undergoing metal laser sintering.

\section{A.2.1.2 Run-time Geometry and Material Updates}

During the course of a simulation, the geometry and material is updated as a function of boundary conditions and subsequent solidification. In the case of the laser exposing the $n^{\text {th }}$ layer, $(n-1)$ layers have been solidified at the regions of laser exposure in the previous layers with an $\boldsymbol{\alpha}^{\prime}$ microstructure whereas the $n^{\text {th }}$ layer is partially solidified from the laser initiation location to its current location based on its melt pool formation and solidification history. This has been done in order to dynamically capture the physical variables such as thermal histories, cooling rates and phase evolutions in the part along with deviation of the part dimensions from its desired design geometry.

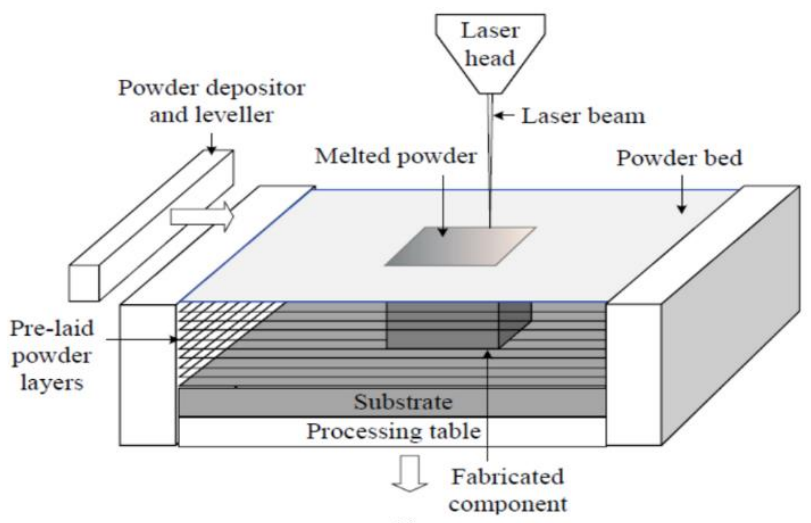

(a)

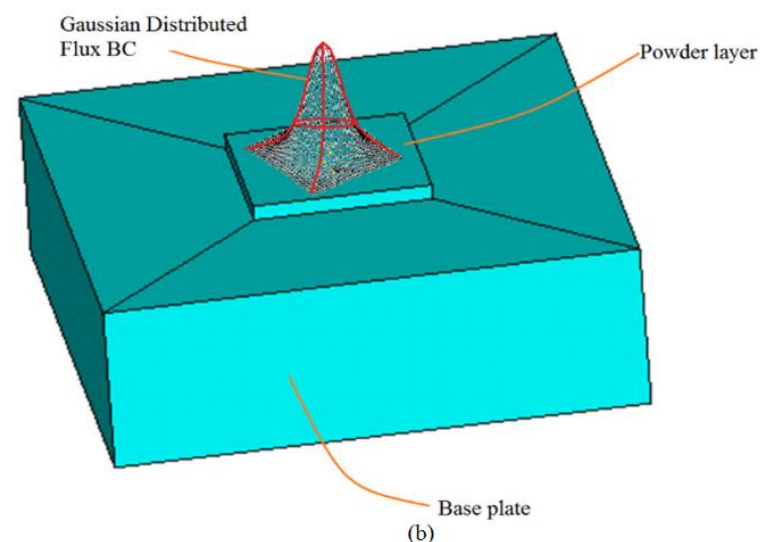

(a)

(b)

Figure A1: (a) Geometry and (b) boundary conditions for metal laser sintering of Ti6Al4V material comprised of a base plate, powder layer and Gaussian laser beam. Forced argon 
in the chamber causes convection which is also considered a boundary condition for the problem, though not shown here.

\section{A.2.1.3 Governing Equations and Boundary Conditions}

The 3 dimensional counterpart of the governing equation is generalized as follows:

$$
\frac{\partial T(\vec{r}, t)}{\partial t}=\frac{1}{c \rho}(\overrightarrow{\vec{k}} \vec{\nabla}) \cdot(\vec{\nabla} T(\vec{r}, t))+Q(\vec{r}, t)
$$

where

$T(\vec{r}, t)=$ Temperature at a position vector $\vec{r}$ in 3 dimensional space and time t.

$\overrightarrow{\vec{k}}=$ Three dimensional material conductivity tensor of order 2 and rank 3 at position vector $\vec{r}$

$c=$ Three dimensional specific heat of the material at position vector $\vec{r}$

$\rho=$ Density of the powder bed at position vector $\vec{r}$

$Q(\vec{r}, t)=$ Inner heat generation at point $\mathrm{x}$ and time $\mathrm{t}$.

Similarly, the three dimensional counterpart of flux is modified as

$$
\vec{q}(\vec{r}, t)=-\overrightarrow{\vec{k}}(\vec{\nabla} T(\vec{r}, t))
$$

where

$\vec{q}(\vec{r}, t)=$ Flux at position vector $\vec{r}$ and time t. 
The spatio-temporal periodicity of the flux boundary condition in 3 dimensions is modified as:

$$
\vec{q}(\vec{r}+\vec{v}(d t), t+d t)=\vec{q}(\vec{r}, t)
$$

The Dirichlet boundary condition considered here is constant temperature at the bottom surface of the base plate as shown in Figure A.2.

$T(0, t)=T_{0}=353 K$

The time-periodic Neumann boundary condition considered here is the laser flux distribution in one dimension:

$$
\vec{q}\left(\left.\vec{r}\right|_{\vec{r} \cdot \vec{e}_{z}=\text { zmax }}, t\right)=\left(\frac{2 P}{\pi r_{\text {laser }}^{2}}\right) \exp \left(\frac{-2\left|\left(\vec{r}-\vec{v} t-\vec{r}_{0}\right)\right|_{\vec{r} \cdot \vec{e}_{z}=z \max } \mid}{r_{\text {laser }}{ }^{2}}\right)
$$

where $P=$ Laser beam power $=180 \mathrm{~W}$

$r_{\text {laser }}=$ Laser beam spot size incident perpendicular to the length $(l=100 \mathrm{~mm})=100 \mu \mathrm{m}$

$\vec{v} t=$ Displacement by the laser beam from the left end of the bar with a speed $(v=$ $1200 \mathrm{~mm} / \mathrm{s}$ ) at time instant $t$

$\vec{r}_{0}=$ Initial position vector of the laser spot on the exposed powder surface

$\left.\right|_{\vec{r} . \vec{e}_{z}=z \max }=$ Condition for the laser flux to always hit the top surface of the powder bed

| $\mid=2^{\text {nd }}$ norm of the included vector

The initial condition for the bar considered here is constant temperature $T_{0}$.

$T(\vec{r}, 0)=T_{0}=353$ Kelvins 


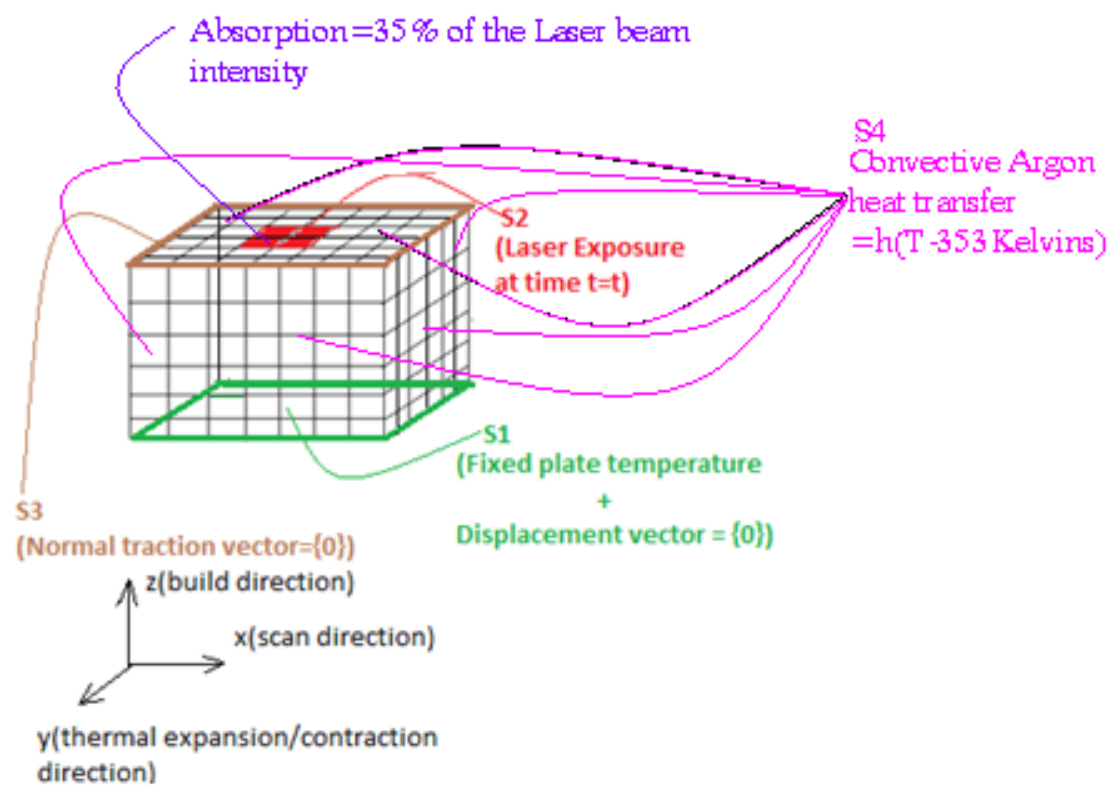

Figure A.2: Surface boundary conditions for the metal laser sintering problem which includes convection, laser flux and fixed temperature boundary conditions

\section{A.2.1.4 Element Formulations}

Traditional thermal solid brick finite element formulations are provided in the literature [160-162]. Latent heats have been added to the brick element as follows:

Metallic alloys such as Ti6Al4V have a range of temperatures at which the solid to liquid transition takes place. These temperatures are in the range of 1877K to 1933K [159]. Hence, the global thermal flux vector $\left\{R_{Q}\right\}_{p}$ is modified in the range of these temperatures:

$$
\left\{R_{Q}\right\}_{p}=\left\{R_{Q}\right\}_{p}-\rho L_{\text {fusion }} \frac{\{M\}_{p}}{\Delta t} * \frac{\left(\{T\}_{p}-T_{s}\right)}{\left(T_{L}-T_{S}\right)}
$$

where 
$\{T\}_{p}=$ Temperature vector for 'p' DOFs

$T_{S}=$ Solidus temperature of the material $=1877 \mathrm{~K}$

$T_{L}=$ Liquidus temperature of the material $=1923 \mathrm{~K}$

$\{M\}_{p}=\operatorname{sign}\left(\operatorname{sign}\left(\frac{\left.\{T\}_{p}-T_{S}\right)}{\left(T_{L}-T_{S}\right)}\right)+\operatorname{sign}\left(\frac{\left(T_{L}-\{T\}_{p}\right)}{\left(T_{L}-T_{S}\right)}\right)\right)$ where sign denotes the signum

function such that

$$
\operatorname{sign}(x)=\left\{\begin{array}{c}
1 \text { for } x>0 \\
0 \text { for } x=0 \\
-1 \text { for } x<0
\end{array}\right.
$$

.* denotes the direct product (one to one product) vector of two vectors of equal length.

$L_{\text {fusion }}$ denotes the latent heat of fusion and $\Delta t$ denotes the time increment per time step.

Vaporization occurs at a temperature of $3533 \mathrm{~K}$ and is very significant in modeling situations where the energy intensity of the laser is high and the speed of the beam motion is low (causing a huge amount of energy to be pumped into the beam focal spot). The energy is large enough to cause the material to vaporize but not large enough to cause a breakdown in thermal continua. $L_{\text {fusion }}$ in equation 6 is replaced by $L_{v a p o r i z a t i o n}$ in this scenario. Density $(\rho)$ has been used as the density of the liquid at the vaporization temperature.

A.2.2 Asymptotic approximation for localized spatio-temporally periodic boundary condition problems 
The generalized finite element discretized equation is as follows:

$$
\left[C_{\text {thermal }}(T)\right]_{p x p}\{\dot{T}\}_{p}+\left[K_{\text {Thermal }}(T)\right]_{p x p}\{T\}_{p}=\left\{R_{Q}\right\}_{p}
$$

The thermal parameters (specific heat, c, density, $\rho$ and thermal conductivity, $k$ ) are piecewise linear functions of temperature. The matrices $\left[C_{\text {thermal }}(T)\right]_{p x p}$ and $\left[K_{\text {Thermal }}(T)\right]_{p x p}$ can be expanded in the convergent Taylor series as:

$$
\begin{aligned}
{\left[C_{\text {thermal }}\right]_{p x p} } & =\left(\left[C_{\text {thermal }}\right]_{p x p}\right)_{T=353 \text { Kelvins }}+\frac{d\left[C_{\text {thermal }}\right]_{p x p}}{d\{T\}}\left(\{T\}_{p}-353\right) \\
& +\frac{d^{2}\left[C_{\text {thermal }}\right]_{p x p}}{2(d\{T\})^{2}}\left(\{T\}_{p}-353\right)^{2} \\
& +\mathcal{O}\left(\frac{d^{3}\left[C_{\text {thermal }}\right]_{p x p}}{6(d\{T\})^{3}}\left(\{T\}_{p}-353\right)^{3}\right) \\
{\left[K_{\text {thermal }}\right]_{p x p} } & =\left(\left[K_{\text {thermal }}\right]_{p x p}\right)_{T=353 \text { Kelvins }}+\frac{d\left[K_{\text {thermal }}\right]_{p x p}}{d\{T\}}\left(\{T\}_{p}-353\right) \\
+ & \mathcal{O}\left(\frac{d^{2}\left[K_{\text {thermal }}\right]_{p x p}}{2(d\{T\})^{2}}\left(\{T\}_{p}-353\right)^{2}\right)
\end{aligned}
$$

where

$$
\begin{gathered}
\mathcal{O}\left(\frac{d^{3}\left[C_{\text {thermal }}\right]_{p x p}}{6(d\{T\})^{3}}\left(\{T\}_{p}-353\right)^{3}\right) \rightarrow 0 \\
\mathcal{O}\left(\frac{d^{2}\left[K_{\text {thermal }}\right]_{p x p}}{2(d\{T\})^{2}}\left(\{T\}_{p}-353\right)^{2}\right) \rightarrow 0
\end{gathered}
$$

Back substituting equations (8-10) in (7) we get 


$$
\begin{aligned}
\left(\left[C_{\text {thermal }}\right]_{p x p}\right)_{T} & =353 \text { Kelvins } \\
& \{\dot{T}\}_{p}+\frac{d\left[C_{\text {thermal }}\right]_{p x p}}{d\{T\}}\left(\{T\}_{p}-353\right)\{\dot{T}\}_{p} \\
& +\frac{d^{2}\left[C_{\text {thermal }}\right]_{p x p}}{2(d\{T\})^{2}}\left(\{T\}_{p}-353\right)^{2}\{\dot{T}\}_{p} \\
& +\left(\left[K_{\text {thermal }}\right]_{p x p}\right)_{T=353 \text { Kelvins }}\{T\}_{p} \\
& +\frac{d\left[K_{\text {thermal }}\right]_{p x p}}{d\{T\}}\left(\{T\}_{p}-353\right)\{T\}_{p}=\left\{R_{Q}\right\}_{p}
\end{aligned}
$$

The prescribed flux vector is:

$$
\left\{R_{Q}\right\}_{p}=\left\{R_{Q}\right\}_{p} \delta\left(\frac{r_{\text {laser }}}{\sqrt{2}}\right)\left(\vec{r}-\vec{r}_{q}-\vec{v} t\right)
$$

Equation (11) can be further grouped for zeroth and higher order terms as follows:

$$
\begin{gathered}
\left(\left(\left[C_{\text {thermal }}\right]_{p x p}\right)_{T=353 \text { Kelvins }}\{\dot{T}\}_{p}+\left(\left[K_{\text {thermal }}\right]_{p x p}\right)_{T=353 \text { Kelvins }}\{T\}_{p}\right) \\
+\left(\frac{d\left[C_{\text {thermal }}\right]_{\text {pxp }}}{d\{T\}}\{\dot{T}\}_{p}+\frac{d^{2}\left[C_{\text {thermal }}\right]_{p x p}}{2(d\{T\})^{2}}\left(\{T\}_{p}-353\right)\{\dot{T}\}_{p}\right. \\
\left.+\frac{d\left[K_{\text {thermal }}\right]_{p x p}}{d\{T\}}\{T\}_{p}\right)\left(\{T\}_{p}-353\right)-\left\{R_{Q}\right\}_{p}=\{0\}_{p}
\end{gathered}
$$

The zeroth order problem can be first solved for the entire domain as follows:

$$
\left(\left[C_{\text {thermal }}\right]_{p x p}\right)_{T=353 \text { Kelvins }}\{\dot{T}\}_{p}+\left(\left[K_{\text {thermal }}\right]_{p x p}\right)_{T=353 \text { Kelvins }}\{T\}_{p}=\left\{R_{Q}\right\}_{p}
$$

Once the temperature vector $\{T\}_{p}$ is obtained then the problem is re-solved with a higher order corrector for selective $\{T\}_{p} \gg 353$ since $\left(\{T\}_{p}-353\right) \rightarrow\{0\}_{p}$ for those $\{T\}_{p} \rightarrow 353$ K. 
It has been observed using the one dimensional analog of the problem that the nonlinear thermal behavior is constricted to the region $\vec{r}=\vec{r}_{q}+\vec{v} t+4 r_{\text {laser }} \hat{r}$ where $\hat{r}$ is the superficial arbitrary radial unit vector with origin at the center of the laser spot. Henceforth, a FFD-AMRD strategy has been followed with refinement enwrapping the region in the near neighborhood of the laser spot centered at $\vec{r}=\vec{r}_{q}+\vec{v} t$.

\section{A.2.3 Locally enriched Dynamic Mesh (FFD-AMRD framework)[162]}

The locally enriched dynamic mesh has been demonstrated as shown in figures A.3 and A.4. This meshing strategy provides significant benefits for solving the metal laser 


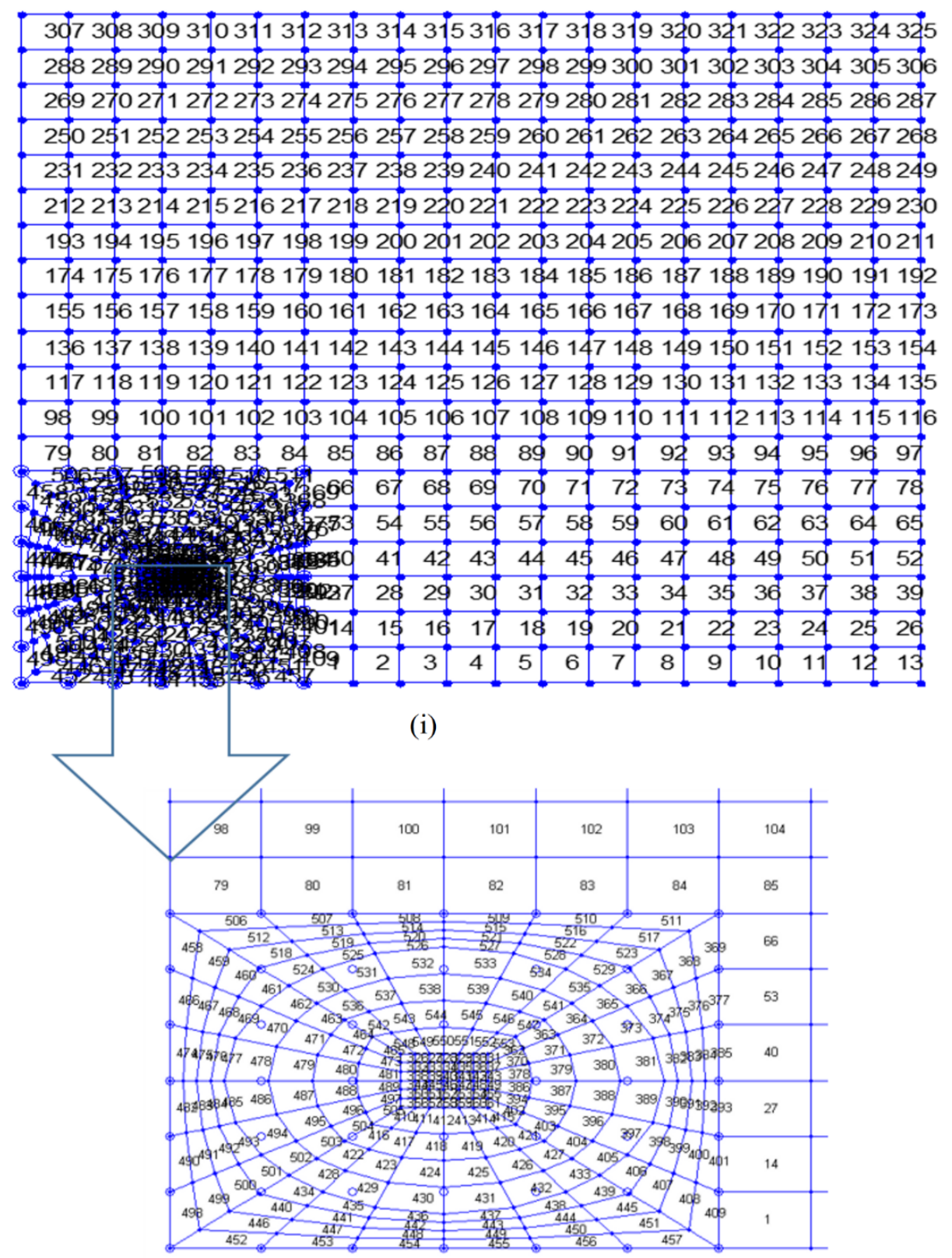

(ii)

Figure A.3: (i) Dynamically enriched mesh at an arbitrary initiation co-ordinate $(3,3)$ with its blown-up fine mesh in (ii). The element and node numbers can be automatically assigned to the mesh in the developed mesh generation tool. Only the $\mathrm{x}-\mathrm{y}$ plane is shown for clarity. The mesh is 3 dimensional in nature. 


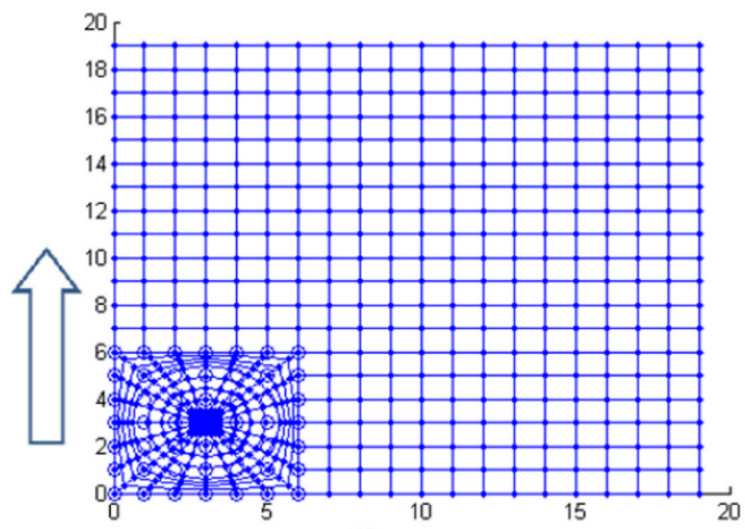

(a)
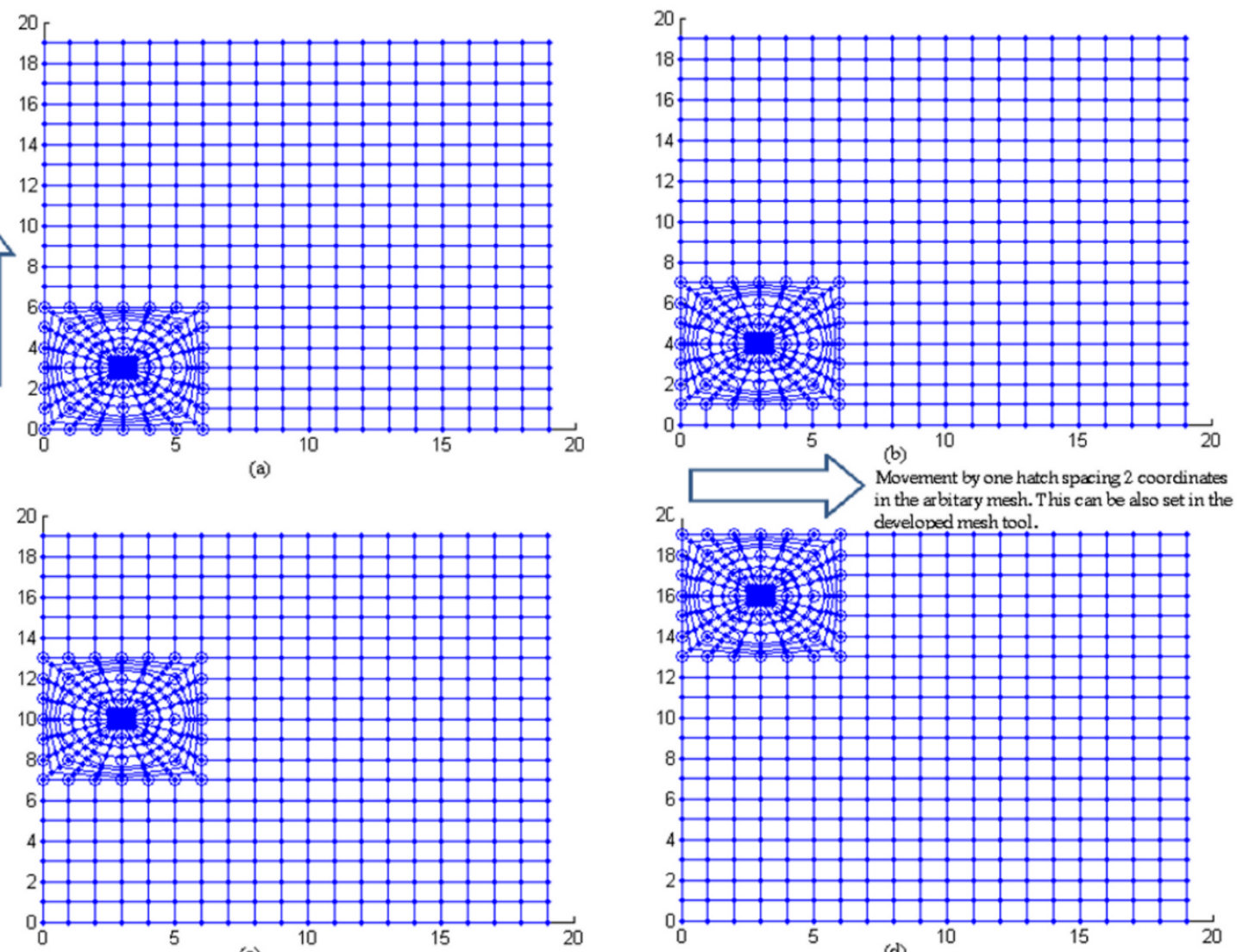

(c)
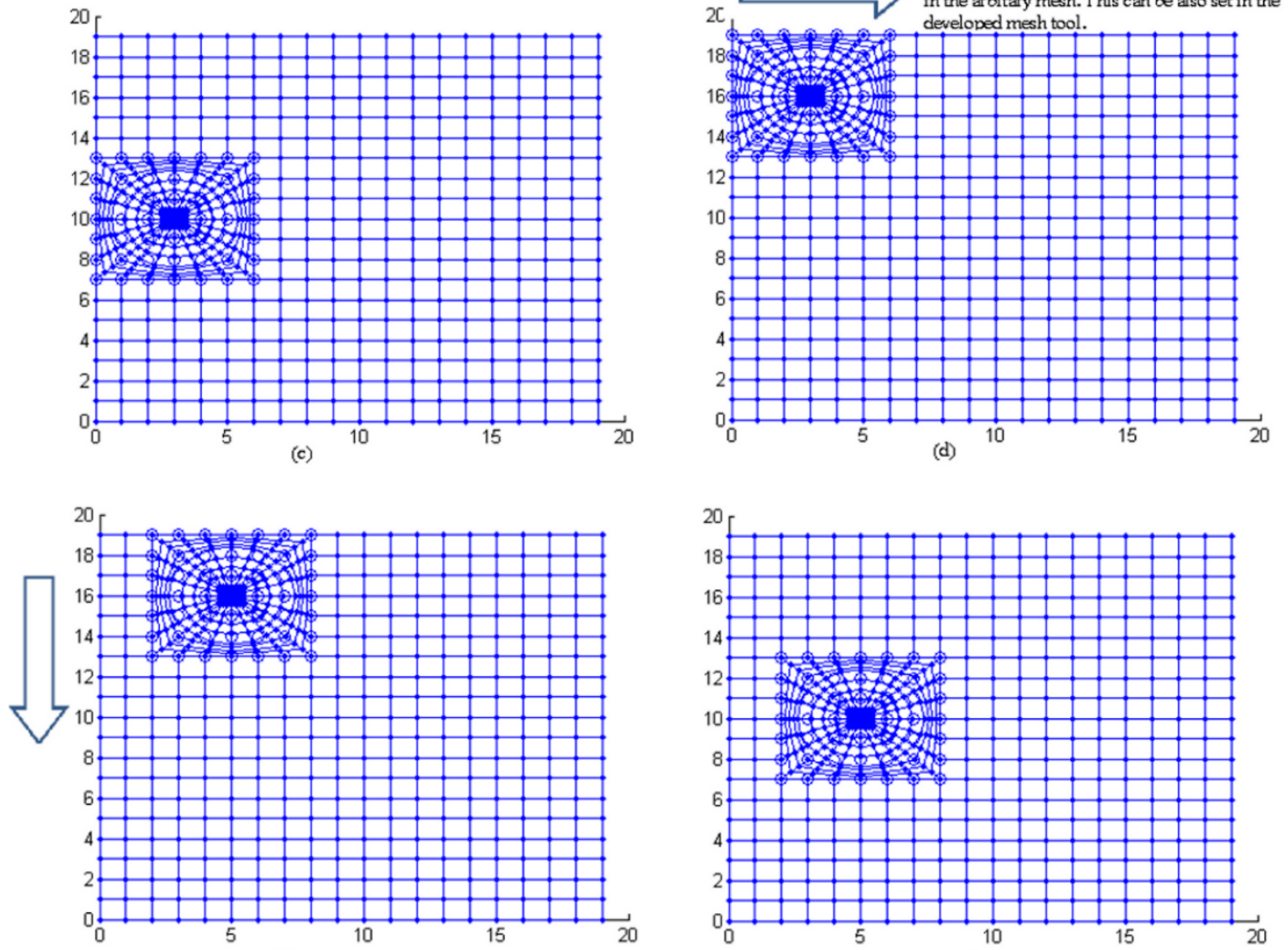

(e)

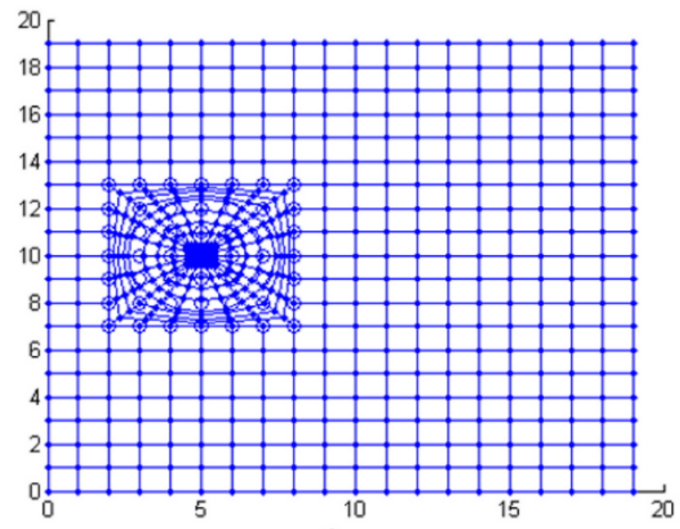

(f)

Figure A.4: (a-f) shows the movement of the dynamic mesh with the laser heat source in the $x-y$ plane of the metal laser sintering machine. The arrow shows the direction of heat source motion. 
sintering thermal problem with increased accuracy in the vicinity of laser exposure where high gradients are anticipated and with required fidelity as radial distance increases away from the point of laser exposure. Figure A.3 shows the multi-scale nature of the mesh and figure A.4 illustrates the dynamics of mesh movement.

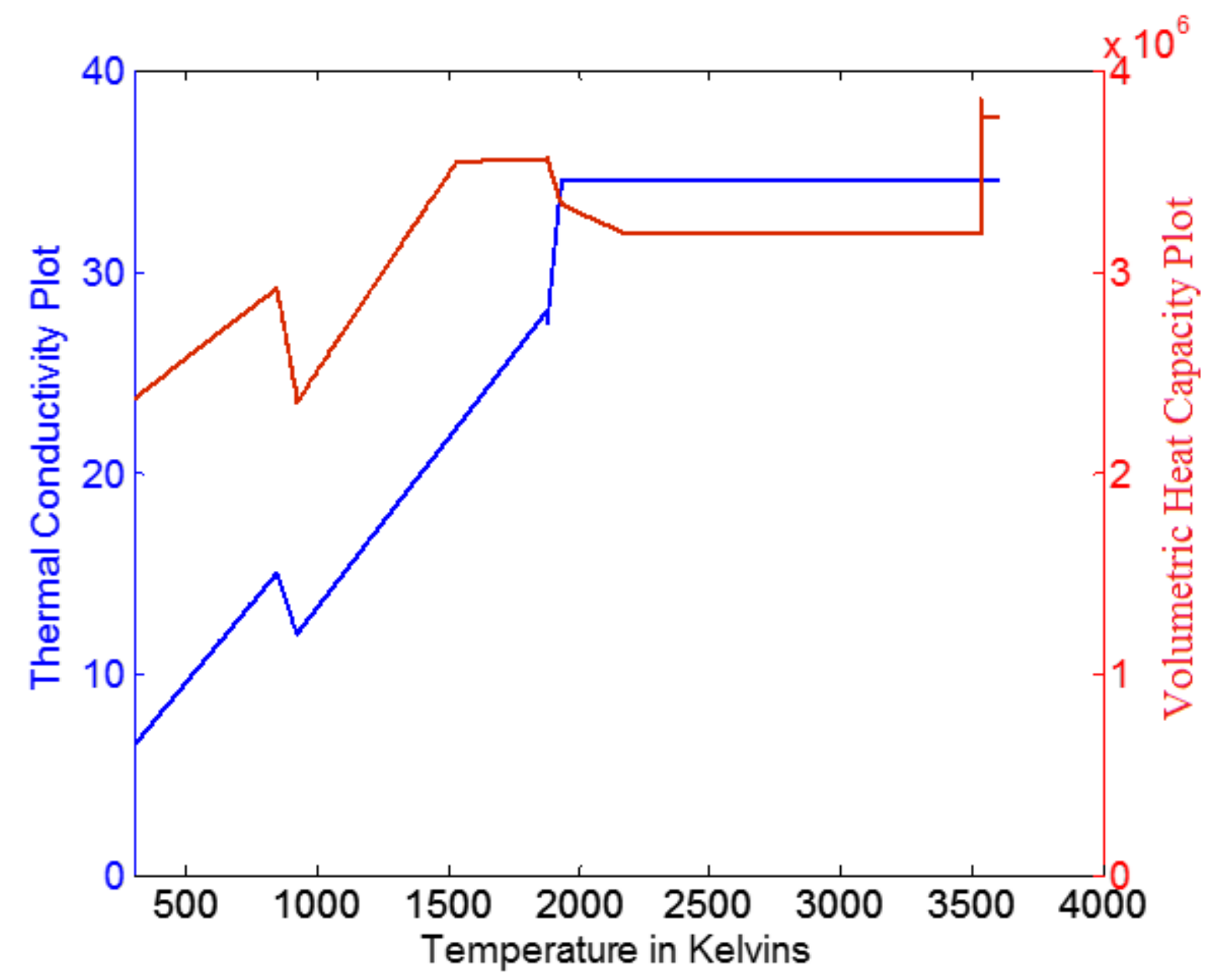

Figure A.5: Temperature dependent conductivity $(k)$ and volumetric heat capacity $(\rho c)$ for Ti6A14V in SI units plotted against temperature in Kelvins. 


\section{A.2.4 Non-linear thermal variables}

Scalar bulk thermal conductivity $(k)$ and volumetric heat capacity $(\rho c)$ vary as a function of temperature [163] as shown in figure A.5. Volumetric heat capacity $(\rho c)$ increases initially with temperature and then goes through a discontinuity in between 900$1100 \mathrm{~K}\left(\alpha^{\prime} \rightarrow \alpha \rightarrow \beta\right.$ phase transformation) and becomes flat at temperatures in the 1500 $1850 \mathrm{~K}$ region. The thermal conductivity also exhibits a similar behavior near $900 \mathrm{~K}$ though it increases until the material reaches its melting point and thereafter becomes constant. Appropriate ratios [163] have been maintained between the bulk and powder thermal characteristics. The powder assumes bulk thermal properties after melting at a temperature higher than $1923 \mathrm{~K}$ (liquidus temperature of Ti6A14V)

\section{A.3. Results and Discussion}

\section{A.3.1 Three dimensional spatio-temporally periodic FEM results}

The thermal contours for linear and non-linear thermal parameters during metal laser sintering of Ti6Al4V are compared in Figure A.6 during a straight pass. It can be clearly seen that the melt pool diameter in the linear scenario overestimates the melt pool diameter compared to the more accurate non-linear solution. In the non-linear scenario, the melt pool diameter is close to the beam diameter $(\sim 100 \mu \mathrm{m})$. In Figure A.7, stable and unstable thermal contours are plotted at various locations within a scan. It can be observed that the thermal contours are unstable near turns as the hatch spacing is less than the length of the melt pool. 


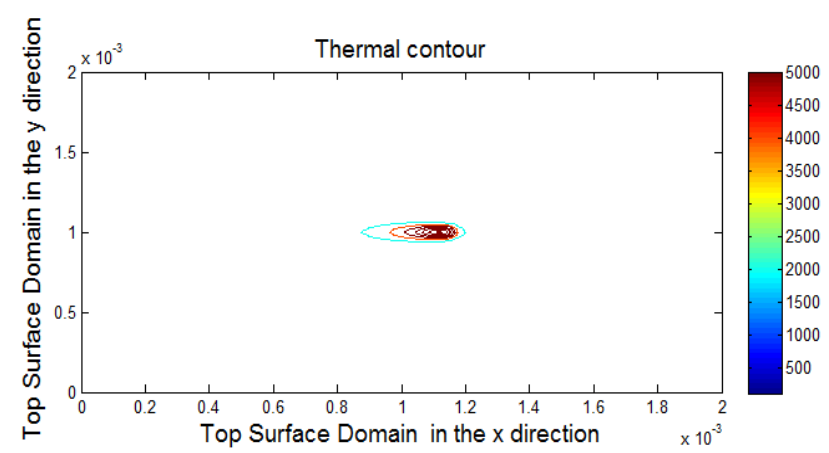

(a)

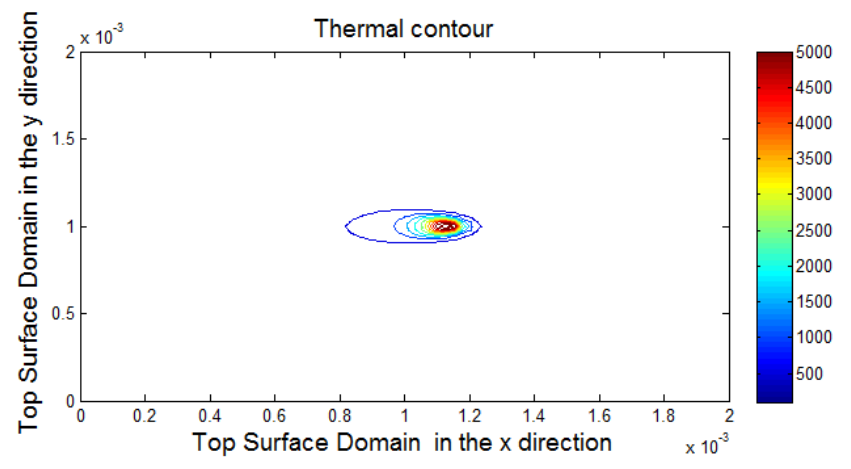

(c)

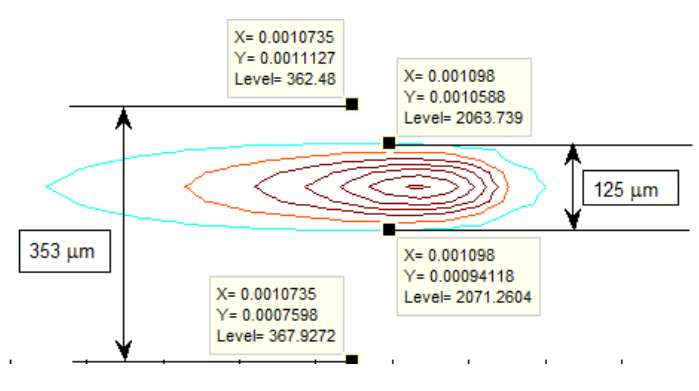

(b)

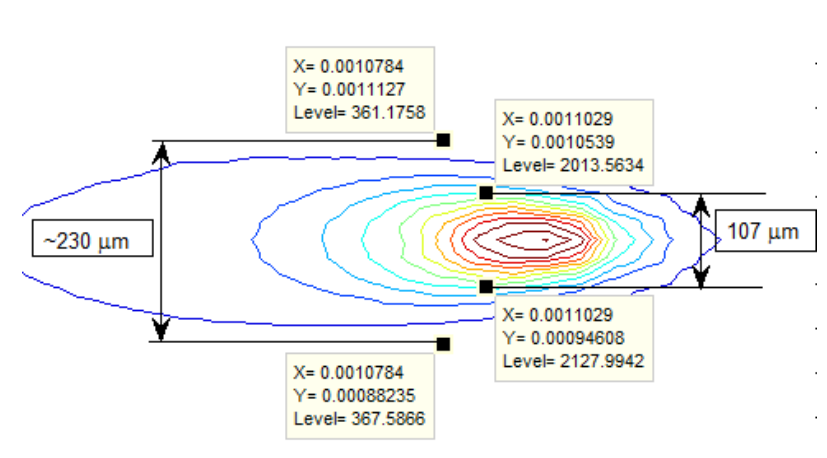

(d)

Figure A.6: Comparison of thermal contours between linear $(a, b)$ and non-linear $(c, d)$ scenarios. The melt pool diameter in (b) is $125 \mu \mathrm{m}$ whereas in (d) it is $107 \mu \mathrm{m}$. The spatial gradient due to laser spot decays steeply in the y direction is $\sim 230 \mu \mathrm{m}$ for the non-linear case compared to $\sim 353 \mu \mathrm{m}$ for the linear case. 


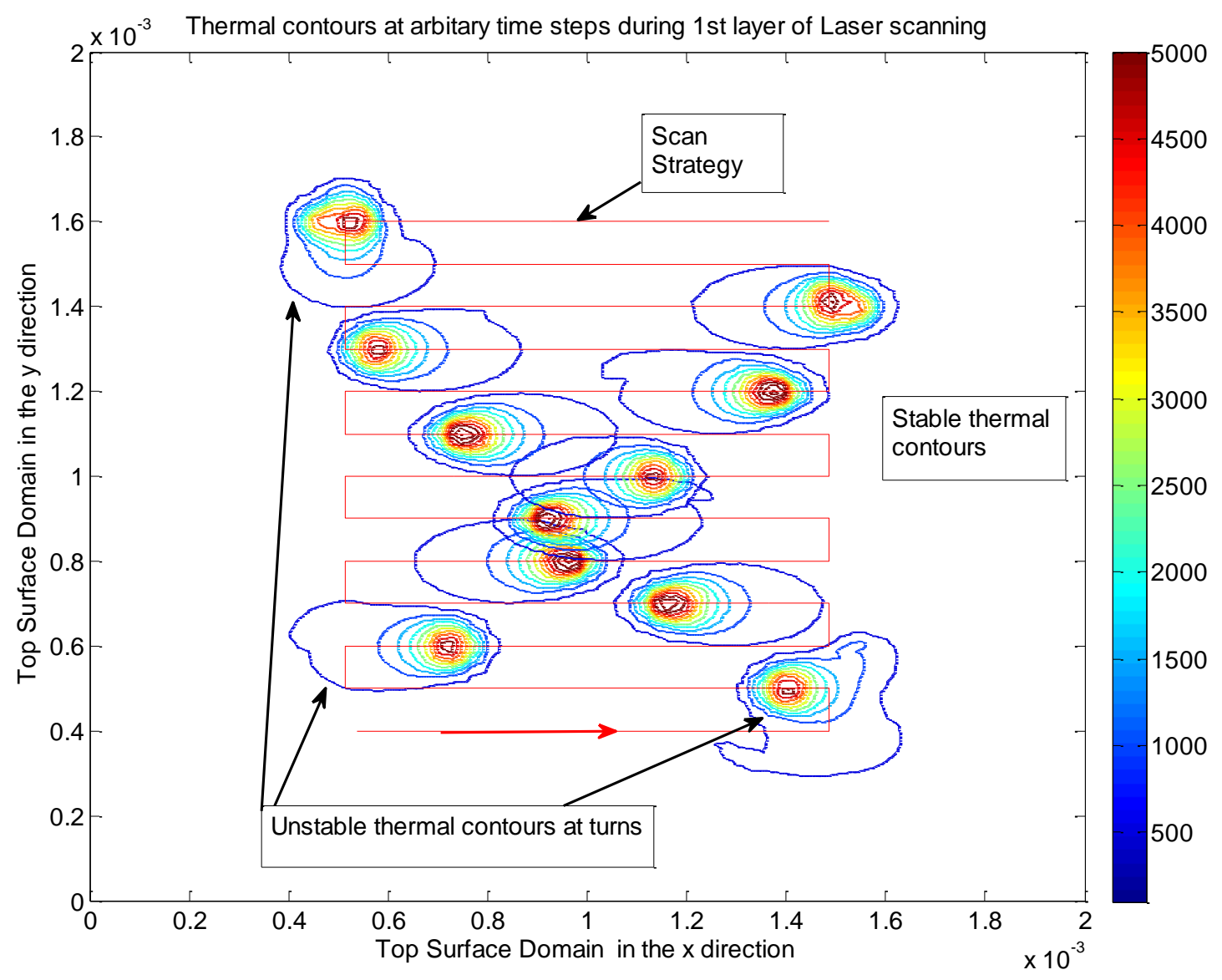

Figure A.7: Stable and Unstable Thermal contours as a function of scan location.

The martensitic $\alpha^{\prime}$ and solidified volume fractions have also been captured as shown in figure A.8. In figure A.8(a), the thermal contours have been plotted as a function of location in the powder bed. Figures A.8(b) and (d) illustrate the region transformed from initial $\alpha$ phase of the powder to $\alpha^{\prime}$ phase and total bulk solidified area respectively. In figures A.8(c) and (e), the evolution of $\alpha^{\prime}$ phase and bulk solidified area as a fraction of total domain area are shown. It can be observed from figure A.8(c) and (e) that the area fraction of martensitic $\alpha^{\prime}$ is slightly greater than the bulk solidified area fraction since the 
powders at the periphery of the part will be transformed to $\alpha$ ' without fully melting, leading to thermally modified but still unmelted powder particles at the periphery of the build slice.
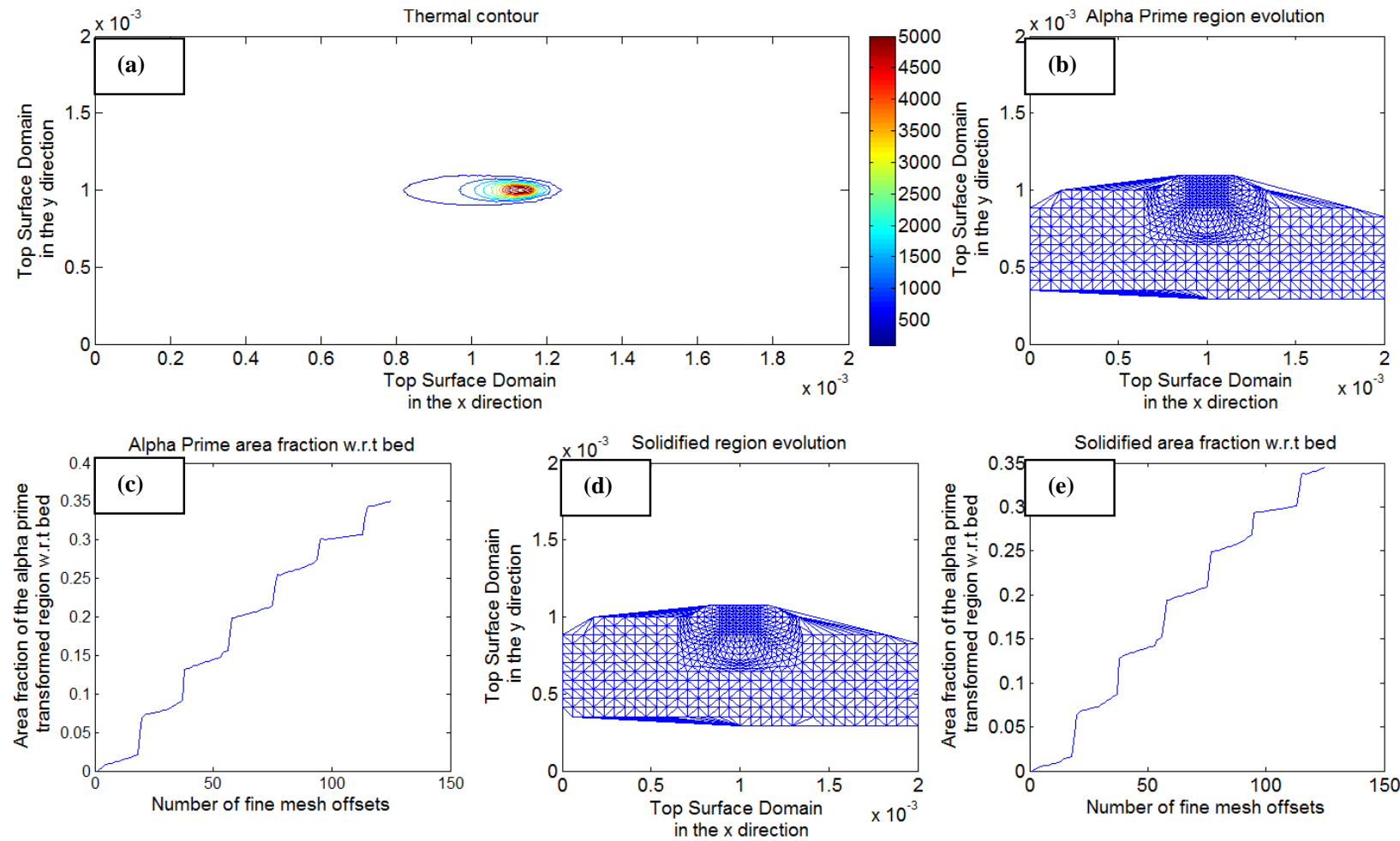

Figure A.8: Plots showing (a) the non-linear thermal distribution, (b) martensitic $\alpha$ ' region evolution, (c) martensitic $\alpha$ ' area phase fraction with respect to the total area of the inset, (d) solidified region evolution, and (e) solidified area phase fraction with respect to the total area of the inset. It can be observed that the solidified area fraction (34.8\%) is slightly smaller than that of the martensitic $\alpha^{\prime}$ area fraction (35.2\%). (Note: the mesh distribution in (b) and (d) are a function of the plotting algorithm used and do have a physical meaning for the plots shown.)

In order to illustrate the effect of unmelted particles at the periphery of a build slice, internal designed defects have been introduced in a continuously tapered cylindrical build as shown 
in figure A.9(a). Figure A.9(b) clearly shows the unmelted particles at the interior periphery of the designed defect. The external periphery of the build was accessible for surface preparation and hence it has been sand blasted to remove loosely bound unmelted particles. The sand blasted external surface is shown in Figure A.9(c).

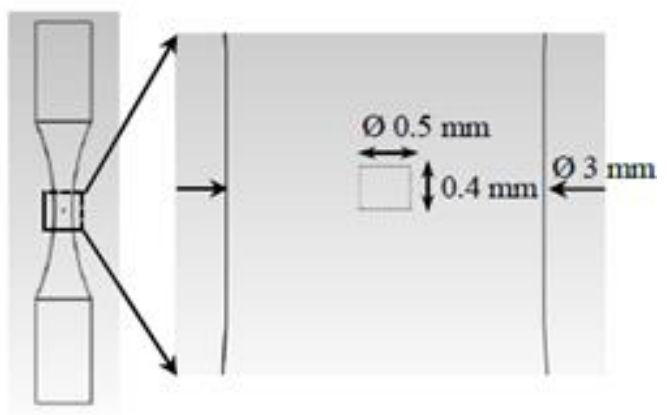

(a)

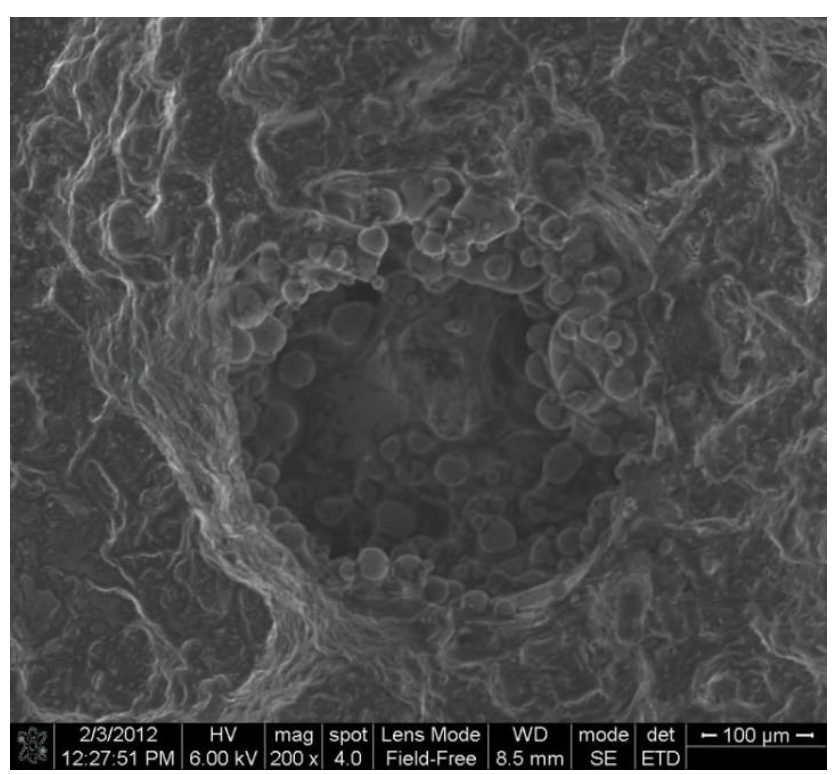

(b)

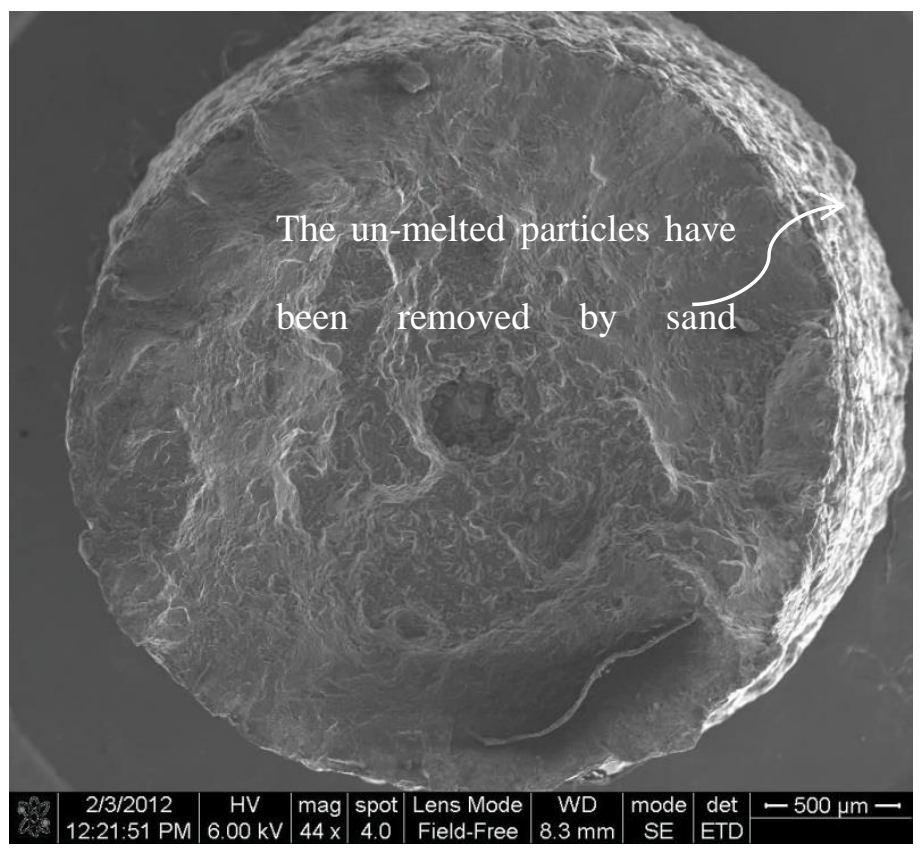

180 
(c)

Figure A.9: (a) Internal defect design, (b) Unmelted particles at an internal surface pertaining to the central cylindrical defect of the build and (c) Unmelted particle removal using sanding at the external surface of the build [164].

The thermal history at the center of the domain held over the base plate has been captured and plotted as a function of time and number of build layers in figure A.10. It can be clearly observed that the heat doesn't significantly affect more than three prior layers, as temperatures seen by the fourth and consequent layers will be much less than the $\beta$ transus temperature.

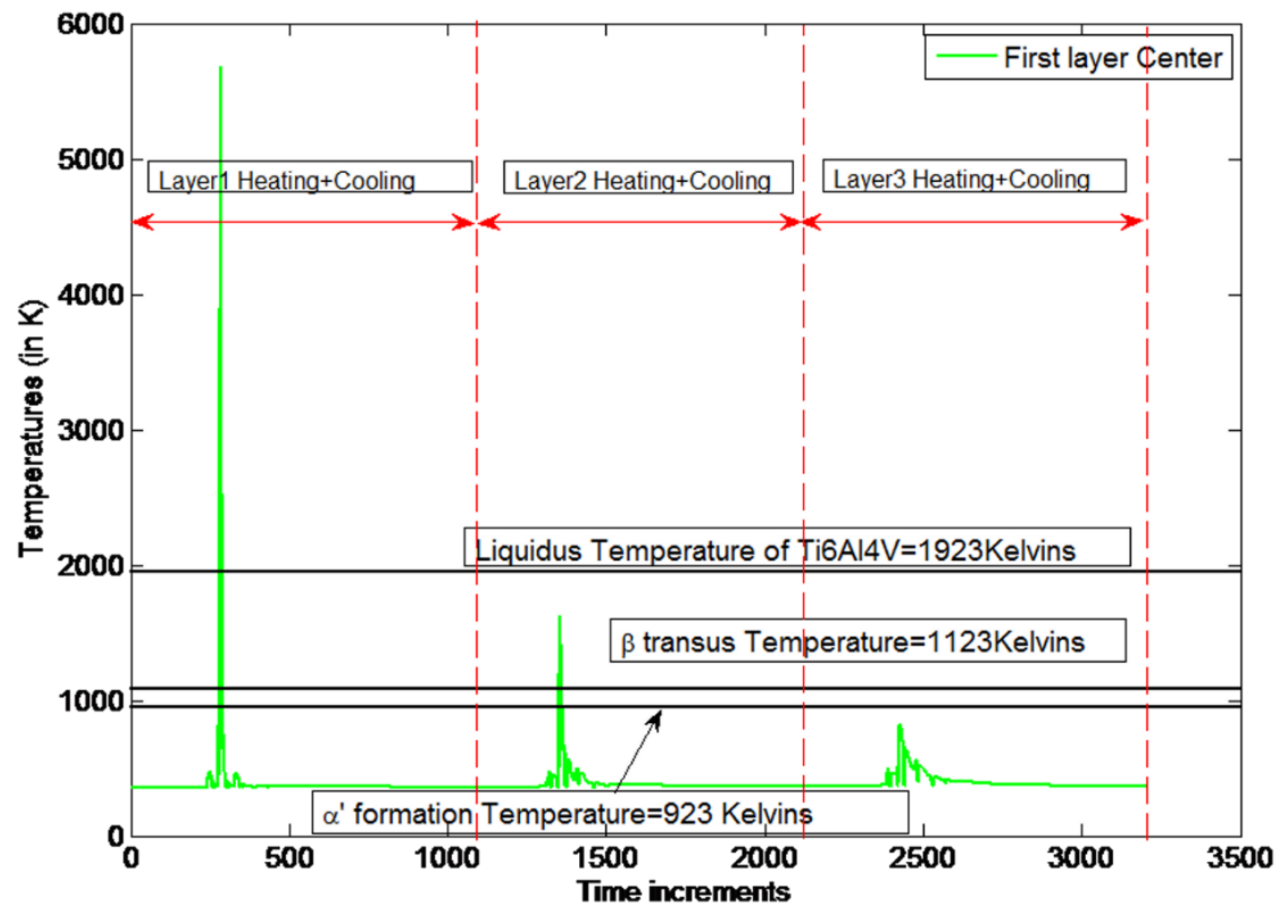

Figure A.10: Thermal history at the center-top of the base plate as additional layers are built over it. 
The thermal contours on subsequent layers at the center of the domain held at the topmost layer of powder are plotted in Figure A.11. It can be clearly observed that the thermal contours become more and more diffused as the $+\mathrm{z}$ distance of the powder layer (distance from the large thermal mass of the base plate) increases.

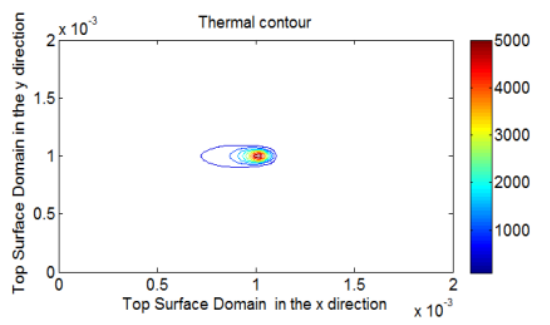

(a)

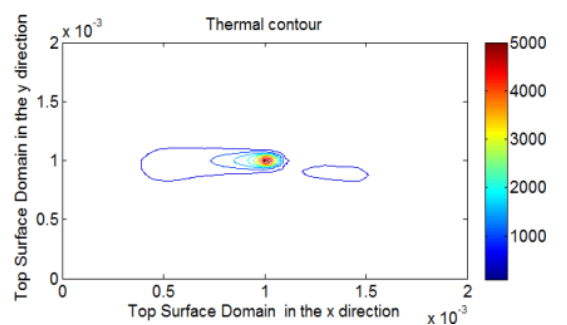

(b)

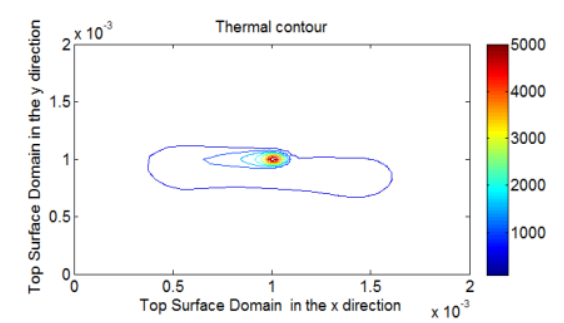

(c)

Figure A.11: Layer-by-layer thermal evolution in metal laser sintering. (a) First layer, (b) Second layer and (c) Third layer.

\section{A.3.2 Thermal and microstructural validations}

The thermal contours were found to be in good agreement with experimentally observed thermal contours for Ti6Al4V when subjected to a laser beam diameter of 200 $\mu \mathrm{m}$ and at a scan speed of $5 \mathrm{~mm} / \mathrm{s}$. The experiment was conducted on a laser process development cell at a research partner facility using a Forward Looking Infra-Red (FLIR) camera. The simulated and experimentally obtained thermal contours match for the same input processing parameters, and are shown in Figures A12 and A13 respectively. A large beam diameter and slow scan speed were chosen to ease the ability of the camera to capture the frames smoothly during the experiment. The slow scan speed also provides an opportunity for the simulation to show its versatility in both high and low scan speed 
regimes. The prior beta grain size should be the lesser of the melt pool width or the hatch spacing, and the fact that it is equal to the hatch spacing indicates that the melt pool width is wider than the hatch spacing as predicted to be $104 \mu \mathrm{m}$ as shown in Figure A.14.

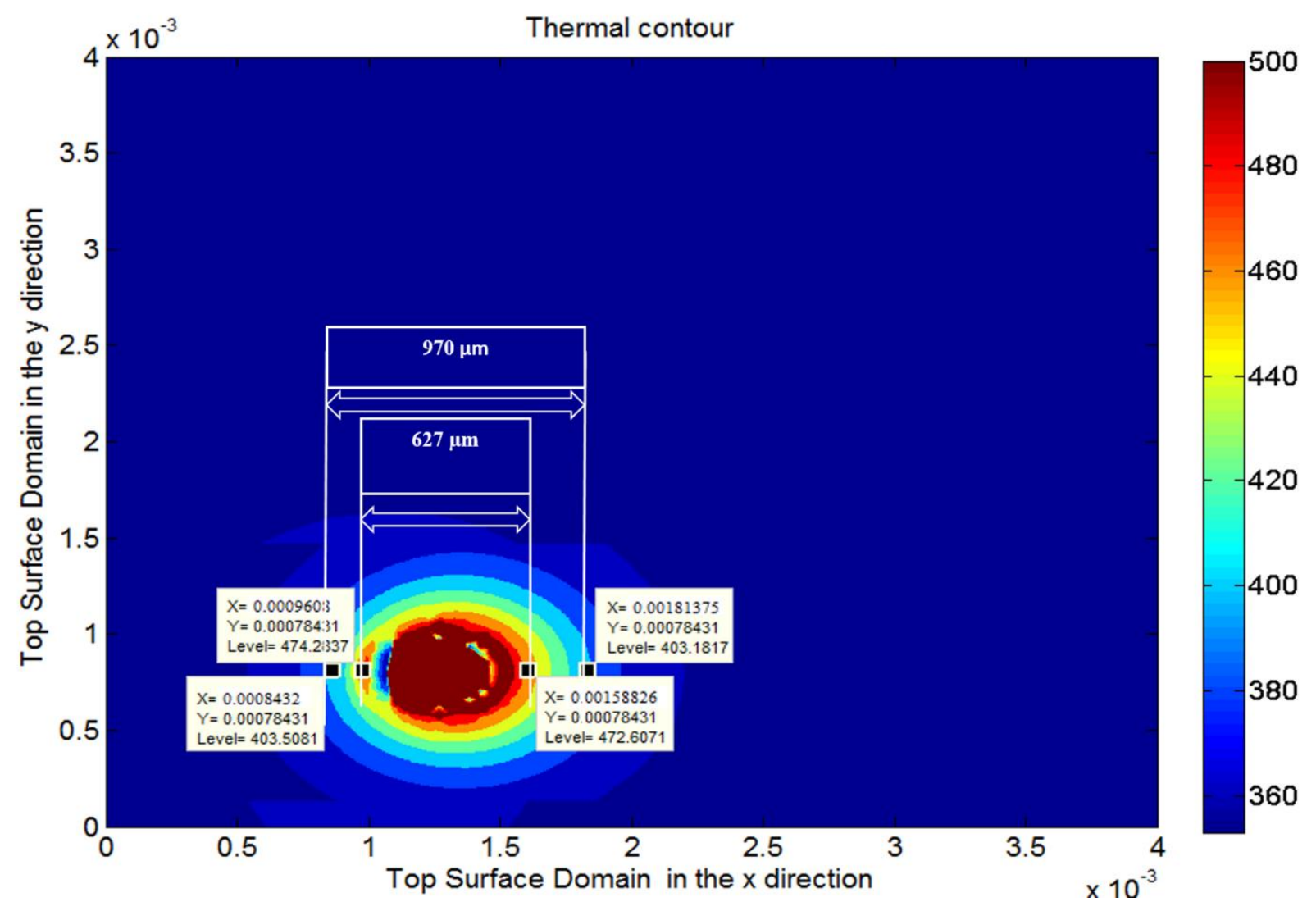

Figure A.12: Simulated thermal contours at the same location and process parameters as those used in the experiment (Figure A.13). Temperature color-bar is shown in Kelvins. 


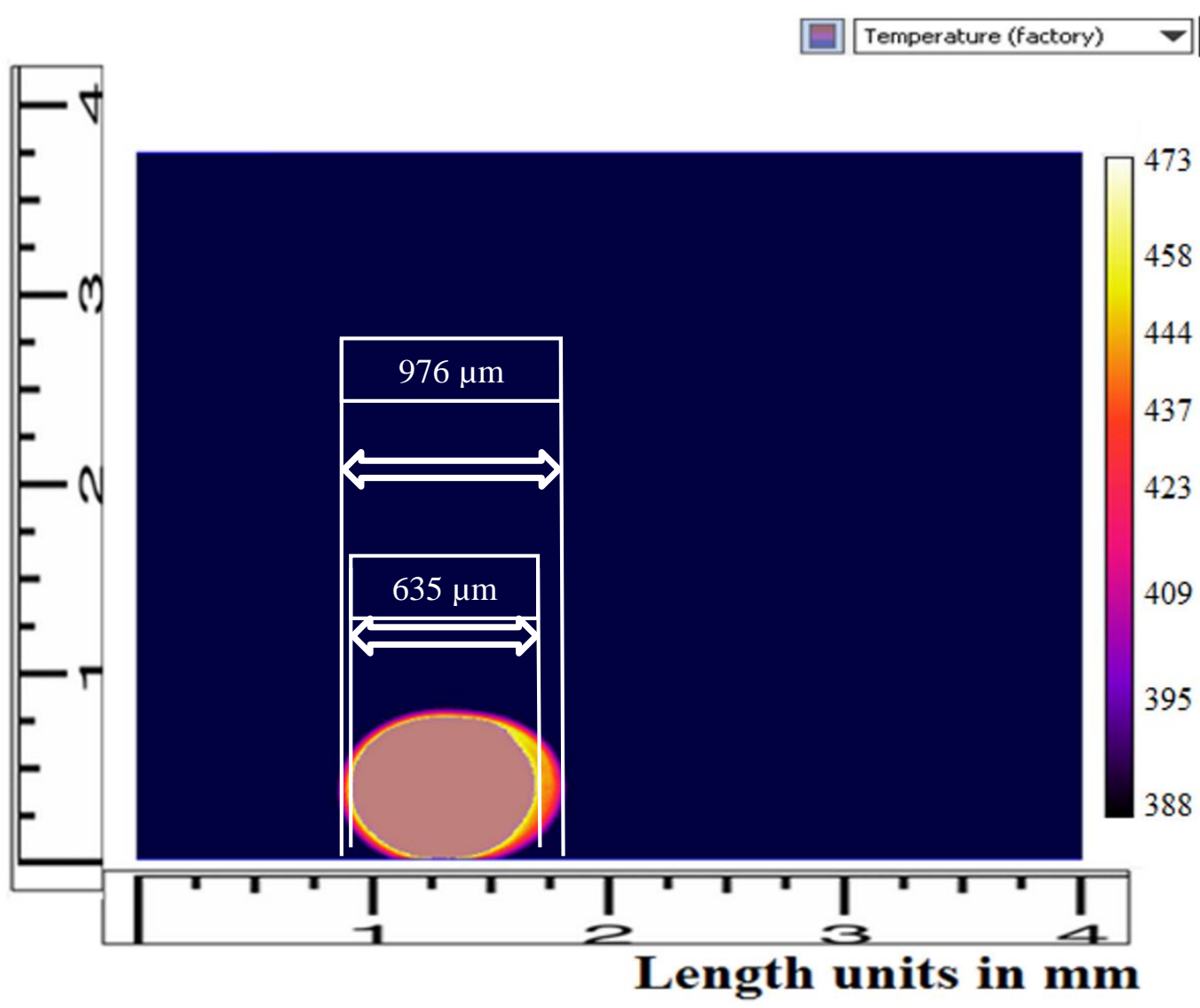

Figure A.13: Experimentally obtained thermal contours 


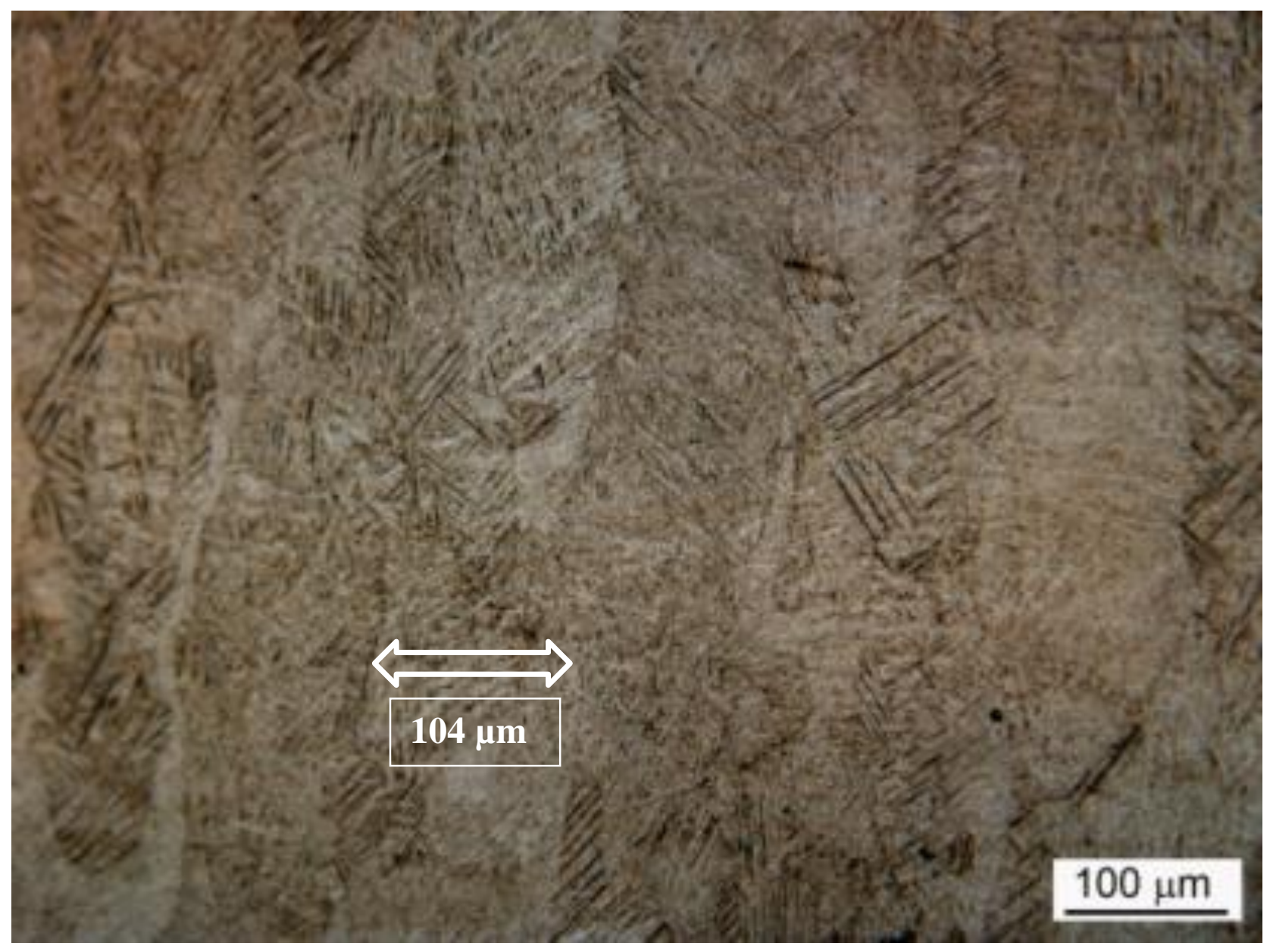

Figure A.14: Transverse prior $\beta$ grain diameter $(\sim 100 \mu \mathrm{m})$.

\section{A.4. Conclusions}

A novel spatial asymptotic approximation has been extended for non-linear problems with spatiotemporally periodic localized boundary conditions. A fully three dimensional finite element analysis with an FFD-AMRD framework for metal laser sintering modeling were developed and used to verify the asymptotic approximation developed in this work.

The proposed framework has been further extended to incorporate the nonlinear thermal behavior of the material along with phase changes such as $\beta \rightarrow \alpha^{\prime}$ during rapid cooling. 
Furthermore, transformation of powder to solidified bulk and its effects on thermal behavior has been tracked. The phase evolution, microstructural predictions, addition of layers on-the-fly and generic scan patterns have been implemented and recorded as a function of time.

The thermal behavior of the metal laser sintering process predicted using the FFD-AMRD simulations was found to be in good agreement with the thermal images captured using a FLIR camera. The microstructural predictions computed were found to be in good agreement with the results obtained from these simulations.

The present computational framework of FFD-AMRD has significant potential for increasing the computational accuracy along with reduction in computational time for thermal process modeling problems similar to metal laser sintering. Future directions for the present work shall be to extend the proposed framework to include a capability to predict the final geometry of the finished part along with computation of the residual stresses. The proposed methodology has potential to be applied to a wide spectrum of problems ranging from additive to traditional manufacturing, problems involving contact mechanics, fracture and delamination problems, gear and dynamic multi-body assemblies and non-linear wave propagation.

\section{$\underline{\text { References }}$}

[145] S. Bremen, W. Meiners and A. Diatlov, Laser Technik Journal 9, 33 (2012).

[146] I. Gibson, D. W. Rosen, and B. Stucker, Additive manufacturing Technologies: Rapid Prototyping to Direct Digital Manufacturing (Springer, NY, 2010).

[147] J. P. Kruth, G. Levy, F. Klocke and T. H. C. Childs, Cirp. Ann-Manuf. Techn. 56, 730 (2007). 
[148] G. N. Levy, R. Schindel and J. P. Kruth, Cirp. Ann-Manuf. Techn. 52, 589 (2003).

[149] J.P. Kruth, B. Vandenbroucke, J. Van Vaerenbergh and P. Mercelis in Benchmarking Of Different S1S/SLM Processes As Rapid Manufacturing Techniques: Proceedings of the 1st International Conference of Polymers and Moulds Innovations, Gent, Belgium, April 20-23 2005.

[150] S. Kumar, J. Mater. 55, 43 (2003).

[151] J.P. Kruth, X. Wang, T. Laoui and L. Froyen, Assembly Autom 23, 357 (2003).

[152] N. K. Tolochko, M. K. Arshinov, A. V. Gusarov, V. I. Titov, T. Laoui and L. Froyen, Rapid Prototyping J. 9, 314 (2003).

[153] X. C. Wang, T. Laoui, J. Bonse, J. P. Kruth, B. Lauwers and L. Froyen, Int. J. Adv. Manuf. Technol. 19, 351 (2002).

[154] A. Simchi, Mat. Sci. Eng. A-Struct. 428, 148 (2006).

[155] I. A. Roberts, C. J. Wang, R. Esterlein, M. Stanford and D. J. Mynors, Int. J. Mach. Tool Manu. 49, 916 (2009).

[156] P. Fischer, V. Romano, H. P. Weber, N. P. Karapatis, E. Boillat and R. Glardon, Acta Mater 51, 1651 (2003).

[157] S. S. Singh, D. Roy, R. Mitra, R. V. Subba Rao, R. K. Dayal, B. Raj and I. Manna, Mat. Sci. Eng. A-Struct. 501, 242 (2009).

[158] K. Zeng, D. Pal, and B. Stucker, Solid Freeform Fabr. Symp. Proc. 23, 796 (2012).

[159] Z. Fan and F. Liou, Numerical Modeling of the Additive Manufacturing (AM) Processes of Titanium Alloy, Titanium Alloys - Towards Achieving Enhanced Properties for Diversified Applications, Dr. A.K.M. Nurul Amin (Ed.), ISBN: 978-953-51-0354-7, InTech (2012), DOI: 10.5772/34848.

[160] T. R. Chandrupatla, and A. D. Belegundu, Introduction to finite elements in engineering (Englewood Cliffs, NJ: Prentice-Hall, 1991) p. 334.

[161] G. P. Nikishkov, Programming Finite Elements in JavaTM (Springer, 2010).

[162] N. Patil, D. Pal, K. Rafi, K. Zeng, A. Moreland, A. Hicks, D. Beeler, and B. Stucker, Journal of Manufacturing Science and Engineering. xxx, xxxxxx (2014). 
[163] See Air Force Research Laboratory Report No. AFRL-rX-WP-TR-2012-0510 (D. Beeler, B. Stucker, D. Pal, N. Patil, and K. Zeng, 2012). Copies may be ordered from the Air Force Research laboratory, Dayton, OH 45433.

[164] H. Gong, Ph.D. thesis, University of Louisville, Louisville, 2013. 


\section{APPENDIX B PERMISSIONS}

This appendix includes all required permissions for publication of the papers presented as chapters and appendices of this dissertation. 


\title{
Re: Permission for including papers in a $\mathrm{PhD}$ thesis
}

\author{
Stucker,Brent < brent.stucker@louisville.edu > \\ Fri 4/25/2014 6:41 AM \\ To:Patil,Nachiket Bapurao <nachiket.patil@louisville.edu >
}

Yes, you may include these in your dissertation.

On Apr 24, 201411.17 PM, "Patil,Nachiket Bapurao" <nbpati01@louisvile.edu> wrote:

Dr. Stucker,

I am preparing my PhD thesis. I am planning to include list of papers as given below in my thesis. I need your kind permission for including these articles in my Thesis. Please reply back to this email with your response.

1. N. Patil, D. Pal, K. Rafi, K. Zeng, A. Moreland, A. Hicks, D. Beeler, and B. Stucker, (2014). A generalized feed forward dynamic adaptive mesh refinement and de-refinement finite element framework for spatiotemporally periodic localized boundary conditions: Application to Metal Laser Sintering, Journal of Manufacturing Science and Engineering. (in review).

2. Pal, D., Patil, N., Rafi, K., Zeng, K., Beeler, D., \& Stucker, B., "A Feed Forward Dynamic Adaptive Mesh Refinement and De-refinement (FFD-AMRD) strategy for problems with non-linear spatiotemporally periodic localized boundary conditions", Journal of Manufacturing Science and Engineering. (in review).

3. Patil, N., Pal, D., \& Stucker, B. (2013). A New Finite Element Solver using Numerical Eigen Modes for Fast Simulation of Additive Manufacturing Processes. Proceedings of the Solid Freeform Fabrication Symposium.

4. Patil, N., Pal, D., \& Stucker, B. E. (2013). An Energy Dissipative Constitutive Model for Multi-Surface Interfaces at Weld Defect Sites in Ultrasonic Consolidation.

5. Patil, N., Pal, D., \& Stucker, B. E., (2014) Intelligent Cholesky decomposition for exchuding the computational overhead of insignificant matrix multiplications (yet to be submitted)

6. Patil, N., Pal, D., \& Stucker, B. E, (2014) A novel crystal plasticity and surface to surface contact simulation framework for simulation of additive manufacturing processes. (yet to be submitted)

Thanks

Nachiket Patil

PhD Candidate, Industrial Engineering, University of Louisville. 


\title{
RE: Permission for including papers in a PhD thesis
}

\author{
Pal,Deepankar <deepankar.pal@louisville.edu> \\ Wed 4/9/2014 11:23 PM \\ To:Patil, Nachiket Bapurao <nachiket.patil@louisville.edu >:
}

To be Dr. Patil,

Yes please with pleasure.

Deepankar

From: Patil,Nachiket Bapurao [nbpati01@louisville.edu]

Sent: Wednesday, April 09, 2014 7:27 PM

To: Pal,Deepankar

Subject: Permission for including papers in a PhD thesis

Dr. Pal,

I am preparing my PhD thesis. I am planning to include list of papers as given below in my thesis. I need your kind permission for including these articles in my Thesis. Please reply back to this email with your response.

1. N. Patil, D. Pal, K. Rafi, K. Zeng, A. Moreland, A. Hicks, D. Beeler, and B. Stucker, (2014). A generalized feed forward dynamic adaptive mesh refinement and de-refinement finite element framework for spatiotemporally periodic localized boundary conditions: Application to Metal Laser Sintering, Journal of Manufacturing Science and Engineering. (in review).

2. Pal, D., Patil, N., Rafi, K., Zeng, K., Beeler, D., \& Stucker, B., "A Feed Forward Dynamic Adaptive Mesh Refinement and De-refinement (FFD-AMRD) strategy for problems with non-linear spatiotemporally periodic localized boundary conditions", Journal of Manufacturing Science and Engineering. (in review).

3. Patil, N., Pal, D., \& Stucker, B. (2013). A New Finite Element Solver using Numerical Eigen Modes for Fast Simulation of Additive Manufacturing Processes. Proceedings of the Solid Freeform Fabrication Symposium.

4. Patil, N., Pal, D., \& Stucker, B. E. (2013). An Energy Dissipative Constitutive Model for Multi-Surface Interfaces at Weld Defect Sites in Ultrasonic Consolidation.

5. Patil, N., Pal, D., \& Stucker, B. E., (2014) Intelligent Cholesky decomposition for exchuding the computational overhead of insignificant matrix multiplications (yet to be submitted)

6. Patil, N., Pal, D., \& Stucker, B. E, (2014) A novel crystal plasticity and surface to surface contact simulation framework for simulation of additive manufacturing processes. (yet to be submitted)

Thanks

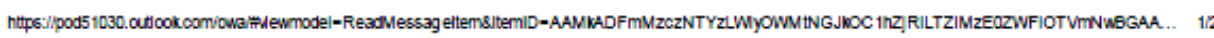

$4 / 11 / 2014$ RE: Permission for Including papers in a PhD thesls - Patl,Nachiket Bapuras

Nachiket Patil

PhD Candidate,

Industrial Engineering,

University of Louisville. 


\title{
RE: Permission for including papers in a PhD thesis
}

\author{
Zeng,Kai \\ Wed 4/9/2014 9:11 PM \\ To:Patil,Nachiket Bapurao <nbpatio@@louisville.edu>;
}

Hello, Nachiket

You have my permission to inchude the articles in your thesis.

\author{
Kai Zeng \\ Department of Industrial Engineering \\ University of Louisville \\ Email kaizeng@buisville.edu \\ Phone: +01(502)8367009 \\ Website: http://www.linkedin.com/in/kaizeng \\ Always stay hungry, and stay foolish! \\ From: Patil,Nachiket Bapurao \\ Sent: Wednesday, April 9, 2014 7:40 PM \\ To: Zeng,Kai \\ Subject: Permission for including papers in a $\mathrm{PhD}$ thesis
}

Dear Kai Zeng,

I am preparing my PhD thesis. I am planning to include list of papers as given below in my thesis. I need your kind permission for including these articles in my Thesis. Please reply back to this email with your response.

1. N. Patil, D. Pal, K. Rafi, K. Zeng, A. Moreland, A. Hicks, D. Beeler, and B. Stucker, (2014). A generalized feed forward dynamic adaptive mesh refinement and de-refinement finite element framework for spatiotemporally periodic localized boundary conditions: Application to Metal Laser Sintering, Journal of Manufacturing Science and Engineering. (in review).

2. Pal, D., Patil, N., Rafi, K., Zeng, K., Beeler, D., \& Stucker, B., "A Feed Forward Dynamic Adaptive Mesh Refinement and De-refinement (FFD-AMRD) strategy for problems with non-linear spatiotemporally periodic localized boundary conditions", Journal of Manufacturing Science and Engineering. (in review).

Thanks

Nachiket Patil

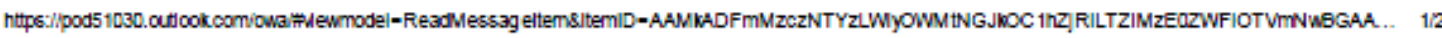

PhD Candidate, Industrial Engineering, University of Louisville. 


\section{Adam Hicks <AdamHicks@mlpc.com> \\ Fri 4/11/2014 2:51 PM \\ To:Patil,Nachiket Bapurao <nbpati01@louisville.edu>;}

You have my permission.

Adam

From: Patil,Nachiket Bapurao [mailto:nbpati01@louisville.edu]

Sent: Wednesday, April 09, 2014 7:46 PM

To: Adam Hicks

Subject: Permission for including papers in a PhD thesis

Dear Adam Hicks,

I am preparing my PhD thesis. I am planning to include list of papers as given below in my thesis. I need your kind permission for including these articles in my Thesis. Please reply back to this email with your response.

1. N. Patil, D. Pal, K. Rafi, K. Zeng, A. Moreland, A. Hicks, D. Beeler, and B. Stucker, (2014). A generalized feed forward dynamic adaptive mesh refinement and de-refinement finite el ement framework for spatiotemporally periodic localized boundary conditions: Application to Metal Laser Sintering, Journal of Manufacturing Science and Engineering. (in review). 2. Pal, D., Patil, N., Rafi, K., Zeng, K., Beeler, D., \& Stucker, B., "A Feed Forward Dynamic Adaptive Mesh Refinement and Derefinement (FFD-AMRD) strategy for problems with non-linear spatiotemporally periodic localized boundary conditions", Journal of Manufacturing Science and Engineering. (in review).

Thanks

Nachiket Patil

PhD Candidate,

Industrial Engineering,

University of Louisville. 


\title{
4/11/2014 RE: Permissian for Including papers In a PhD thesls - Pafli,Nachllet Bapurao \\ RE: Permission for including papers in a PhD thesis
}

\author{
Alleyce Moreland <AlleyceMoreland@mlpc.com> \\ Fri 4/11/2014 3:04 PM \\ To:Patil,Nachiket Bapurao <nbpatio1@louisville.edu>;
}

You have my permission to include these articles in your thesis.

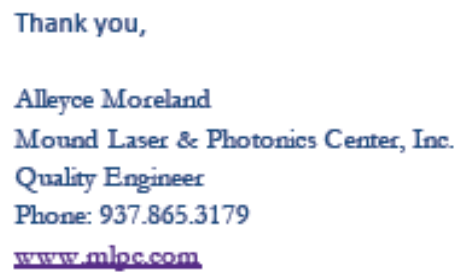

Thank you,

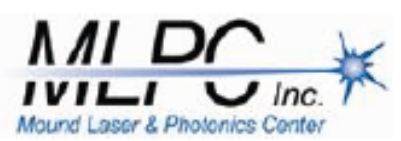

"Effective May 23, 2013-New Mailing \& Shipping Address*

2941 College Drive

Kettering, Ohio 45420

Fromr Patil,Nachiket Bapurao [mailto:nbpati01@louisville.edu]

Sent: Friday, April 11, 2014 2:21 PM

To: Alleyce Moreland

Subject: Permission for induding papers in a PhD thesis

Alleyece,

I am preparing my PhD thesis. I am planning to include list of papers as given below in my thesis. I need your kind permission for including these articles in my Thesis. Please reply back to this email with your response.

1. N. Patil, D. Pal, K. Rafi, K. Zeng, A. Moreland, A. Hicks, D. Beeler, and B. Stucker, (2014). A generalized feed forward dynamic adaptive mesh refinement and de-refinement finite element framework

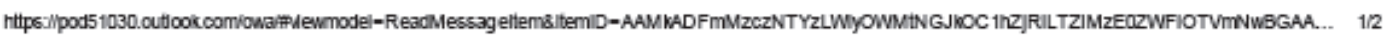

4/11/2014 RE: Permission for Including papers in a PhD thesis - Patl,Nachiket Bapurao

for spatiotemporally periodic localized boundary conditions: Application to Metal Laser Sintering, Journal of Manufacturing Science and Engineering. (in review).

Thanks

Nachiket Patil 


\title{
RE: Permission for including papers in a $\mathrm{PhD}$ thesis
}

\author{
Kevin Hartke <kevinhartke@mlpc.com> \\ Wed 4/9/2014 8:03 PM \\ To:Patil,Nachiket Bapurao <nbpatio1@louisville.edu> \\ Nachiket, \\ David Beeler no longer works for MLPC but these papers were written while he was employed with us and as a \\ result you have permission to use them in your thesis. Thanks.
}

Kevin Hartke

President and COO

2941 College Dr.

Kettering, Ohio 45420

Mound Laser \& Photonics Center Inc.

937-865-3070

Fronr Patil,Nachiket Bapurao [mailto:nbpati01@louisville.edu]

Sent: Wednesday, April 09, 2014 7:50 PM

To: David Beeler

Subject: Permission for including papers in a PhD thesis

Dear David Beeler,

I am preparing my PhD thesis. I am planning to include list of papers as given below in my thesis. I need your kind permission for including these articles in my Thesis. Please reply back to this email with your response.

1. N. Patil, D. Pal, K. Rafi, K. Zeng, A. Moreland, A. Hicks, D. Beeler, and B. Stucker, (2014). A generalized feed forward dynamic adaptive mesh refinement and de-refinement finite element framework for spatiotemporally periodic localized boundary conditions: Application to Metal Laser Sintering, Journal of Manufacturing Science and Engineering. (in review).

2. Pal, D., Patil, N., Rafi, K., Zeng, K., Beeler, D., \& Stucker, B., "A Feed Forward Dynamic Adaptive Mesh Refinement and De-refinement (FFD-AMRD) strategy for problems with non-linear spatiotemporally periodic localized boundary conditions", Journal of Manufacturing Science and Engineering. (in review).

Thanks

Nachiket Patil

PhD Candidate, Industrial Engineering, University of Louisville. 


\title{
$4 / 11 / 2014$ \\ Re: Permission for Including papers in a PhD thesls - Patll,Nachlket Bapurao \\ Re: Permission for including papers in a PhD thesis
}

\author{
Khalid Rafi <khalidrafi@gmail.com> \\ Thu 4/10/2014 10:45 PM \\ To.Patil,Nachiket Bapurao <nbpati01@louisville.edu >
}

Dear Nachiket,

Nice to hear from you and happy to note that you are at the last phase of your PhD.I am very much happy to have these articles in your thesis and thankful for making me to associate with your work.

All the very best.

Thanks and Regards

Rafi

On Wed, Apr 9, 2014 at 7:36 PM, PatilNachiket Bapurao <nbpatio1eilouiavile.edu> wrote:

Dr. H. Khalid Rafi,

I am preparing my PhD thesis. I am planning to include list of papers as given below in my thesis. I need your kind permission for including these articles in my thesis. Please reply back to this email with your response.

1. N. Patil, D. Pal, K. Rafi, K. Zeng, A. Moreland, A. Hicks, D. Beeler, and B. Stucker, (2014). A generalized feed forward dynamic adaptive mesh refinement and de-refinement finite element framework for spatiotemporally periodic localized boundary conditions: Application to Metal Laser Sintering, Journal of Manufacturing Science and Engineering. (in review).

2. Pal, D., Patil, N., Rafi, K., Zeng, K., Beeler, D., \& Stucker, B., "A Feed Forward Dynamic Adaptive Mesh Refinement and De-refinement (FFD-AMRD) strategy for problems with non-linear spatiotemporally periodic localized boundary conditions", Journal of Manufacturing Science and Engineering. (in review).

Thanks

Nachiket Patil

PhD Candidate,

Industrial Engineering,

University of Louisville.

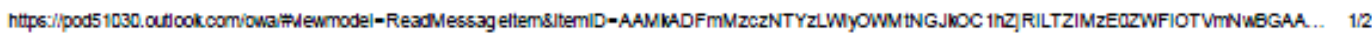

4/11/2014

Re: Permission for Including papers in a PhD thesis - Patll,Nachiket Bapurao

Dr.Khalid Rafil H, PhD

Research Felow

School of Mechanical and Aerospace Engineering

Nanyang Technological University, Singapore

Contact No.

Office: $+65-67904334$

Mobile: $+65-81671837$ 


\section{CURRICULUM VITAE}

\section{Name: Nachiket Patil}

\section{Education:}

$\begin{array}{llll}2014 \text { (Expected) } & \text { PhD, Industrial Engineering } & \text { University of Louisville } \\ 2008 & \begin{array}{l}\text { Master of } \quad \text { Technology } \\ \text { (M.Tech.) }\end{array} & \text { Indian Institute of Technology Bombay } \\ & \text { Engineering } & \text { Structural } & \text { (at Mumbai) } \\ 2006 & \text { Bachelor of Engineering (B.E.), } & \text { Veermata Jijabai Technological Institute } \\ & \text { Civil Engineering } & \text { (Mumbai University) }\end{array}$

Present Position: (January 2011 - present)

Graduate Research Assistant, Industrial Engineering, University of Louisville.

\section{Research Experience:}

\section{Industrial Engineering, University of Louisville}

Position: Graduate Research Assistant.

(June 2011 - Present)

Time efficient Finite Element Methods with specific focus on

- Spatial Homogenization of Dislocation Density Based Finite Element Framework Team: Dr. Deepankar Pal and Dr. Brent Stucker Timeline: June 2011- Present 
- Feed forward Dynamic Adaptive Mesh Refinement for moving time periodic boundary conditions.

Team: Dr. Deepankar Pal and Dr. Brent Stucker

Timeline: July 2012- Present

\section{Mechanical Engineering, Brigham Young University}

Position: Graduate Research Assistant.

(January 2011- May 2011)

Determination of surface and lattice dislocation tensors using high resolution EBSD

Team: Dr. David Fullwood and Dr. Brent L. Adams.

\section{Mechanical and Aerospace Engineering, Utah State University}

(September 2009- December 2010)

Position: General Graduate Assistant.

Lab Rotation(s)

- Fracture Mechanics

- $\quad$ Additive Manufacturing

- Bio Fuels

- Variational Asymptotic Methods for beams and plates analysis

\section{Aerospace Engineering, Indian Institute of Sciences}

(August 2008 - June 2009)

Project Associate

Project title: Large pantographing deformation of cord rubber composites in a Pneumatic tire during tire building and shaping.

Team: Dr. Dineshkumar Harursampath. 


\section{M.Tech. Project:}

Position - Graduating Masters' Student

(August 2007 - July 2008)

Analysis and Design of Composite Sandwich Ship Hull Structure

Team: Dr. Asokendu Samanta (Indian Registrar of Shipping) and Dr. R. S. Jangid (IIT, Bombay).

\section{Teaching Assistance:}

Industrial Engineering, University of Louisville

Fall 2011 - Probability and Statistics for Engineers (IE 360)

Spring 2012- Manufacturing Processes (IE 320)

\section{Mechancial and Aerospace Engineering, Utah State University.}

Fall 2009 — Solid Mechancis (MAE 3040)

Spring 2010 - Numerical Methods (MAE 2450)

\section{Publications:}

1. N. Patil, D. Pal, K. Rafi, K. Zeng, A. Moreland, A. Hicks, D. Beeler, and B. Stucker, (2014). A generalized feed forward dynamic adaptive mesh refinement and derefinement finite element framework for spatiotemporally periodic localized boundary conditions: Application to Metal Laser Sintering, Journal of Manufacturing Science and Engineering. (in review).

2. Pal, D., Patil, N., Rafi, K., Zeng, K., Beeler, D., \& Stucker, B., “A Feed Forward Dynamic Adaptive Mesh Refinement and De-refinement (FFD-AMRD) strategy for problems with non-linear spatiotemporally periodic localized boundary conditions", Journal of Manufacturing Science and Engineering. (in review).

3. Pal, D., Patil, N., \& Stucker, B. (2013). Erratum: A study of subgrain formation in Al 3003 H-18 foils undergoing ultrasonic additive manufacturing using a dislocation 
density based crystal plasticity finite element framework". Journal of Applied Physics, 114(1).

4. Patil, N., Pal, D., Anam, MD., Gong, H., Gu, H., Dilip, S., and Stucker, B., (2014) "Predictive modeling capabilities for dimensional accuracy and surface finish in Metal Laser Melting based Additive Manufacturing”, 2014 ASPE Spring Topical Meeting.

5. Patil, N., Pal, D., \& Stucker, B. (2013). A New Finite Element Solver using Numerical Eigen Modes for Fast Simulation of Additive Manufacturing Processes. Proceedings of the Solid Freeform Fabrication Symposium.

6. Patil, N., Pal, D., \& Stucker, B. E. (2013). An Energy Dissipative Constitutive Model for Multi-Surface Interfaces at Weld Defect Sites in Ultrasonic Consolidation. Proceedings of the Solid Freeform Fabrication Symposium.

7. Nikoukar, M., Patil, N., Pal, D., \& Stucker, B. (2013). Methods for Enhancing the Speed of Numerical Calculations for the Prediction of the Mechanical Behavior of Parts Made Using Additive Manufacturing. Proceedings of the Solid Freeform Fabrication Symposium.

8. Pal, D., Patil, N., Nikoukar, M., Zeng, K., Kutty, K. H., \& Stucker, B. E. (2013). An Integrated Approach to Cyber-Enabled Additive Manufacturing using Physics based, Coupled Multi-scale Process Modeling.

9. Pal, D., Patil, N., \& Stucker, B. E. (2012). Prediction of mechanical properties of Electron Beam Melted Ti6A14V parts using dislocation density based crystal plasticity framework.

10. Zeng, K., Pal, D., Patil, N., \& Stucker, B. (2013). A New Dynamic Mesh Method Applied to the Simulation of Selective Laser Melting. Proceedings of the Solid Freeform Fabrication Symposium.

11. Zeng, K., Patil, N., Gu, H., Gong, H., Pal, D., Starr, T., \& Stucker, B. (2013). Layer by Layer Validation of Geometrical Accuracy in Additive Manufacturing processes. Proceedings of the Solid Freeform Fabrication Symposium.

12. Patil, N., Namdev, R., \& Samanta, A. (2009). Applicability of ANSYS elements in analyzing sandwich vessels. Paper presented at the International Conference in Ocean Engineering (IOCE-2009), IIT - Madras. 
13. Patil N., Kamble Y., and Sangle K., (2010), "A review on instabilities during deformation: void growth and coalescence " International Conferance on Innovative World of Structural Engineering, Govt. College of Engineering, Aurangabad.

\section{Academic Achievements:}

- National Institute of Standards and Technology (NIST) Graduate Tuition Fellowship ( January $2013-2014$ ).

- Industrial Engineering Graduate Student Award - 2014

- Secured All India Rank 15 (99.85 percentile) in Civil Engineering Master's Qualifying Examination GATE 2006 .

- Secured $7^{\text {th }}$ rank in Associate Degree Final Year in Civil Engineering in Maharashtra State. 